Florida International University FIU Digital Commons

$11-19-1993$

\title{
Performance assessment of prosthetic heart valves using orifice area formulae and the energy index method
}

Flavio Ballerini Souza Campos

Florida International University

DOI: $10.25148 /$ etd.FI15110714

Follow this and additional works at: https://digitalcommons.fiu.edu/etd

Part of the Biomechanical Engineering Commons

\section{Recommended Citation}

Souza Campos, Flavio Ballerini, "Performance assessment of prosthetic heart valves using orifice area formulae and the energy index method" (1993). FIU Electronic Theses and Dissertations. 2432.

https://digitalcommons.fiu.edu/etd/2432 


\title{
FLORIDA INTERNATIONAL UNIVERSITY
}

$$
\text { Miami, Florida }
$$

\section{Performance Assessment of Prosthetic Heart Valves Using Orifice Area Formulae and the Energy Index Method}

\author{
A thesis submitted in partial satisfaction of the \\ requirements for the degree of \\ MASTER OF SCIENCE \\ IN
} MECHANICAL ENGINEERING by

Flavio Ballerini Souza Campos 
TO: Gordon Hopkins, Dean

College of Engineering and Design

This thesis, written by Flavio Ballerini Souza Campos, and entitled Performance Assessment of Prosthetic Heart Valves Using Orifice Area Formulae and the Energy Index Method, having been approved in respect to style and intellectual content, is referred to you for judgement.

We have read this thesis and recommend that it be approved.

\author{
M. Ali Ebadian
}

Genady P. Cherepanov

Richard T. Schoephoerster, Major Professor

Date of Defense: 11/19/1993

The thesis of Flavio B. Soura Campos is approved.

Gordon Eopkins, Dean

College of Engineering and Design

Richard dampbell, Dean

Division of Graduate Studies

Florida International University, 1993 


\section{DEDICATION}

This work is dedicated to all the angels without whom it would never be. Some of these angels are portrayed here.

It all started and ended with him. It was he that reminded me not to forget the three most important words at the end of the paper. It was he that, through his appreciation for the infinite beauty of the human body, and his brilliance in describing it in engineering terms, steered my attention and interest in this direction.

Another angel encouraged me not to quit school when I did not know who I was. There was he, who, graduating before me, gave me something to aim for. The father was a great example, for he worked hard, every day. Other angels worked in the most unimaginable ways: pretending they were impressed with the equipment or teaching my hands to bring something to the world that previously was not.

Sitting high, he was the best example I ever had of patience, understanding, and overall humanness. Being young and well accomplished on one hand, and well rounded on the other, helped me see that it all is possible.

Through his infinite wisdom, some sense, out of what apparently had none, came to be; and part of his peace is now mine. Her infinite kindness let me know that even if it seemed that it would never be, it was only because it already was, and had always been. Of course, none of this would have happened, were it not for their love for one another. 


\section{ACRNOWIEDGEMNTS}

It would be impossible to remember and mention every one that contributed to the success of this work. It would also be unnecessary, since this whole work was dedicated to those that contributed with their hearts, expecting nothing in return.

On a more formal level, however, we thank the following manufacturers for supplying sample valves for the conduction of these experiments:

St. Jude Medical

Medtronic Hall

Carpentier-Edwards

Partial financial support of this work was provided for by the American Heart Association. 


\section{ABSTRACT OF THE THESIS}

Performance Assessment of Prosthetic Heart Valves Using Orifice Area Formulae and the Energy Index Method by

Flavio Ballerini Souza Campos Florida International University, 1993 Miami, Florida

Professor Richard T. Schoephoerster, Major Professor

Valve function is commonly assessed by effective orifice area (EOA) estimates using equations derived from conservation of mass and energy. Errors have been found with the method due to difficulties in determining the valve's coefficient of discharge (Cd). The Cd, a factor that corrects the EOA for losses in the valvular wake region, has been shown previously to vary with the Reynolds number and valve geometry. In this study, a Cardio-Vascular Duplicator (CVD) is used to determine the Cd for three types of mitral valves, operating in modes ranging from normal to severely stenotic. Since orifice area methods do not account for regurgitant flow, the energy index (EI) method is derived and used in experiments with an aortic valve. Results show that the EI method is more powerful than the EOA because a single quantitative parameter is attributed to each valve, taking into account regurgitant, leakage and pressure losses. 


\section{CONTENTS}

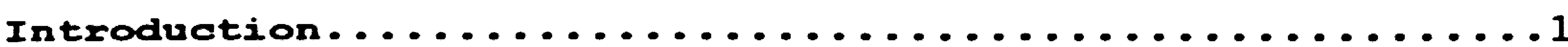

Chapter 1: Physiological Aspects of Relerance...........3

The Human Cardiovascular system...............

The Human Heart........................

Anatomical Description of Heart Valves..........11

Common Malfunctions......................12

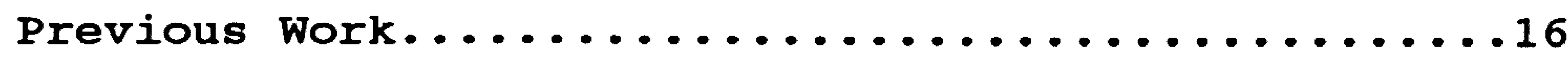

Chapter 2: The Effective Orifice Area................19

Formulation of Problem and Method of Solution......19

Experimental Apparatus....................25

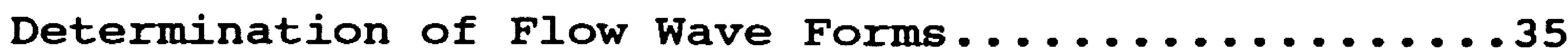

Method of Implementation and Meaning of Valve

Condition............................42

Experimental Protocol....................46

Description of valves tested...............59

Results.............................61

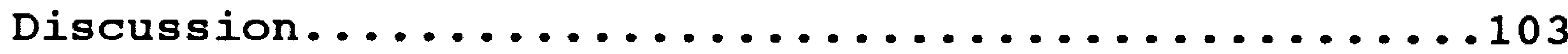

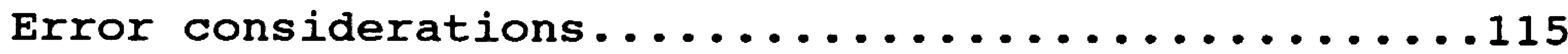

Suggestions for Future Work.................119

Chapter 3: The Power Inder......................125

Introduction............................. 25

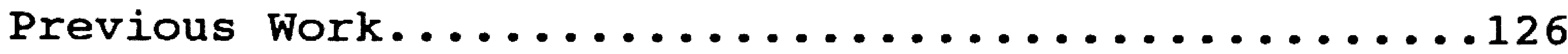

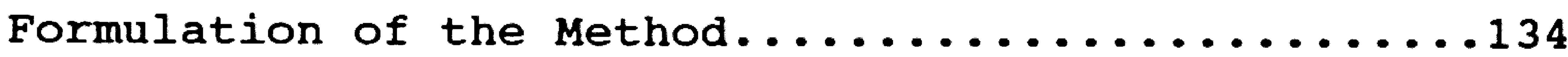

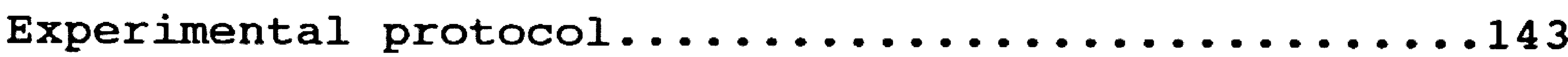


Results...............................146

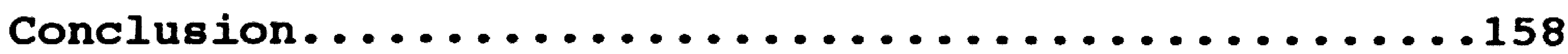

Closing Remarks........................... 154

References.

.159

$\nabla i \boldsymbol{i}$ 


\section{IIST OF FIGURES}

Figure $1 \ldots \ldots \ldots \ldots \ldots \ldots \ldots \ldots \ldots \ldots \ldots \ldots \ldots \ldots \ldots \ldots$

Figure $2 \ldots \ldots \ldots \ldots \ldots \ldots \ldots \ldots \ldots \ldots \ldots \ldots \ldots \ldots \ldots$

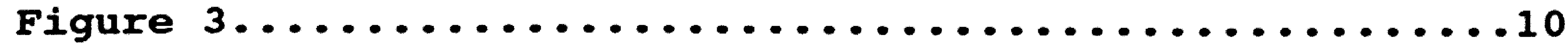

Figure $4 \ldots \ldots \ldots \ldots \ldots \ldots \ldots \ldots \ldots \ldots \ldots \ldots \ldots \ldots \ldots \ldots$

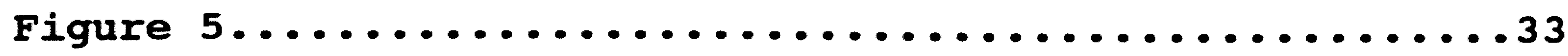

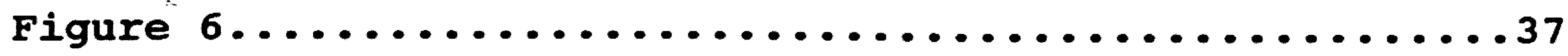

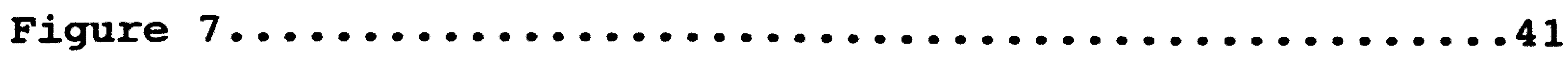

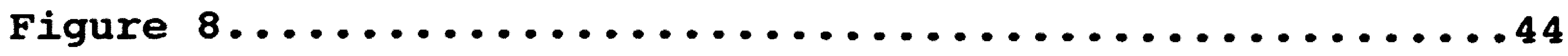

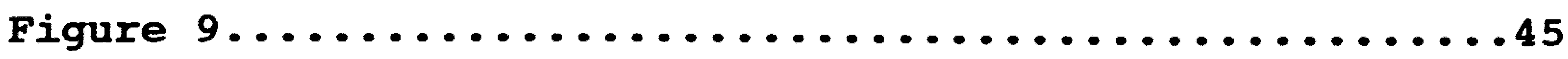

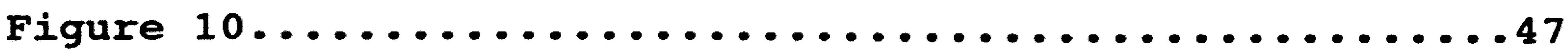

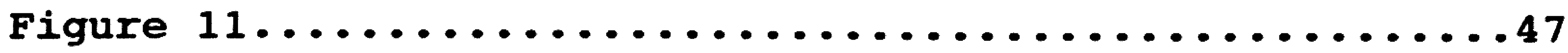

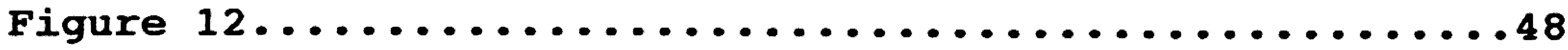

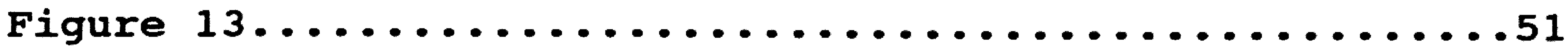

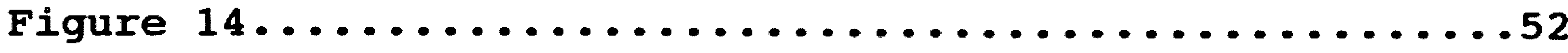

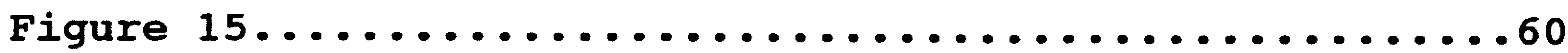

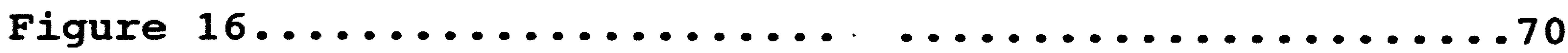

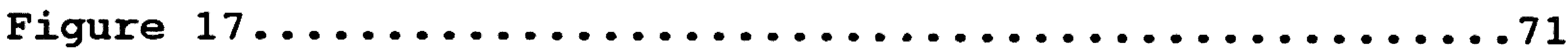

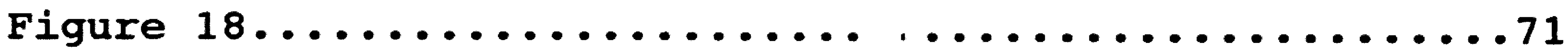

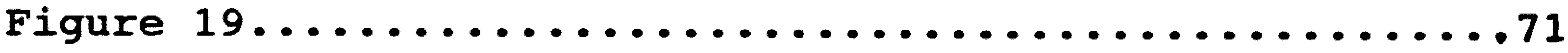

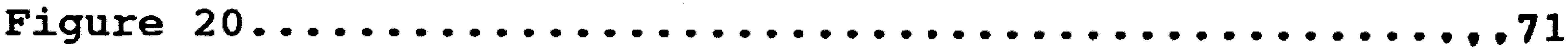

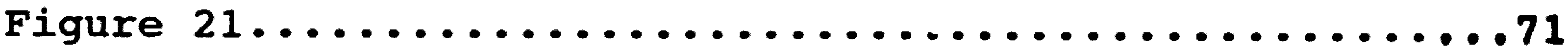

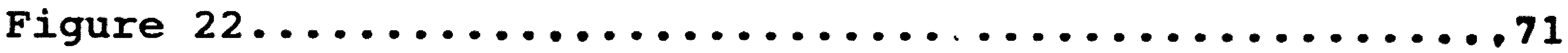

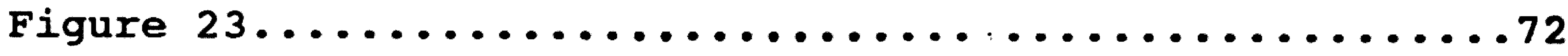

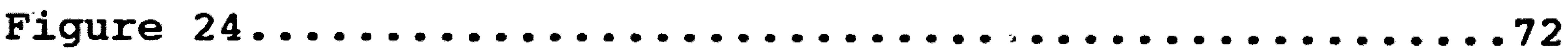




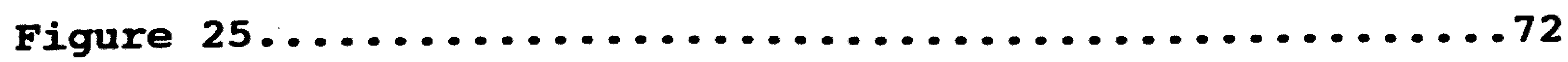

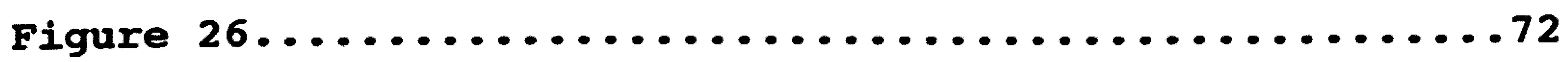

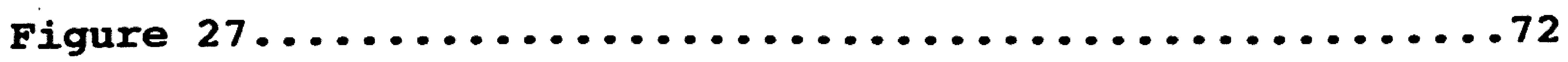

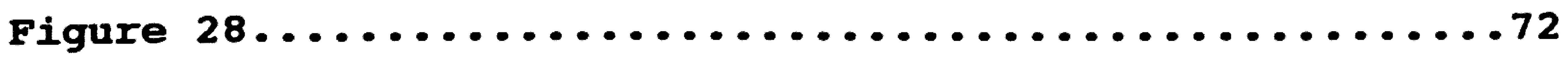

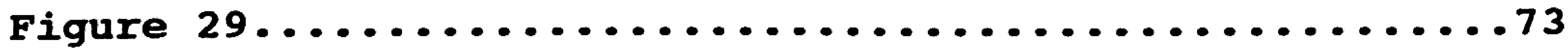

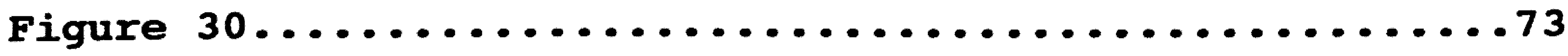

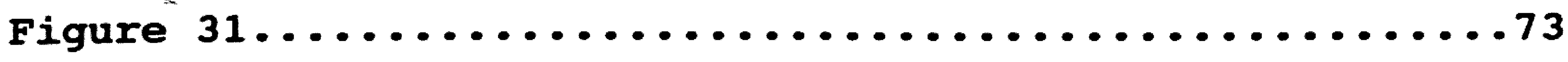

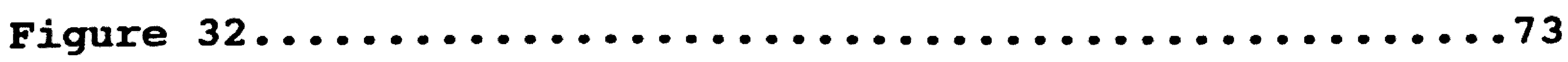

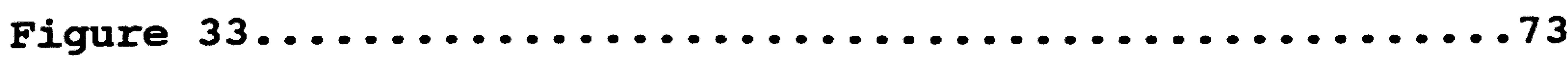

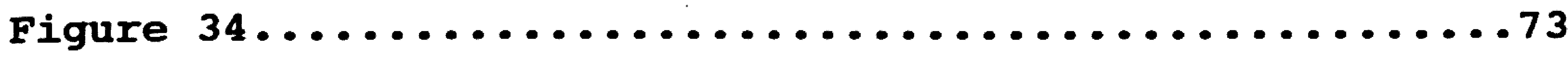

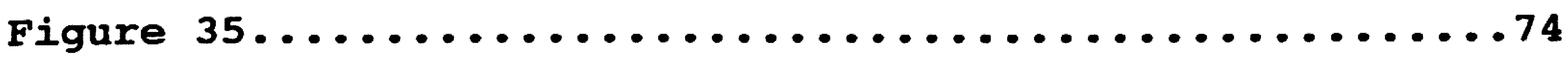

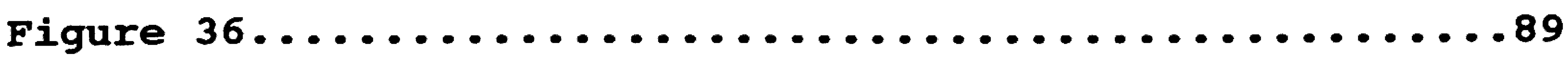

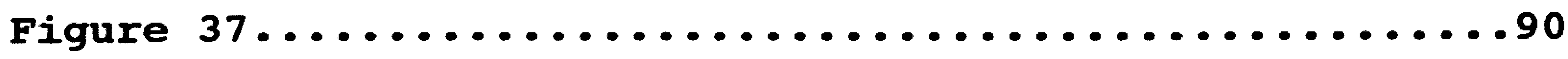

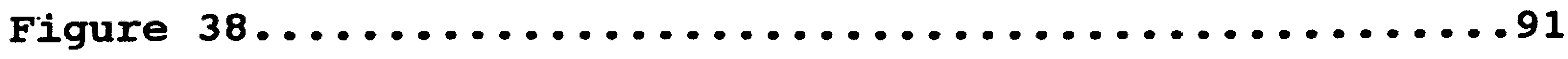

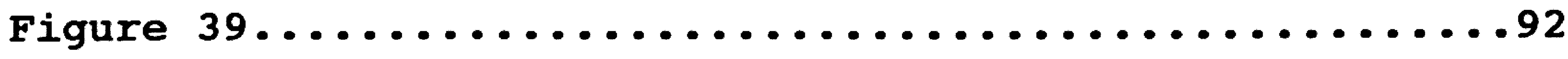

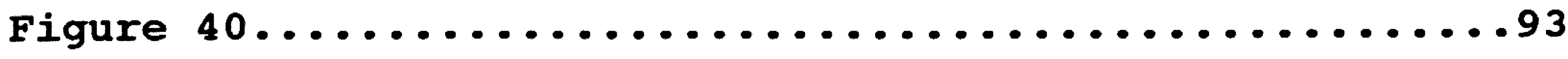

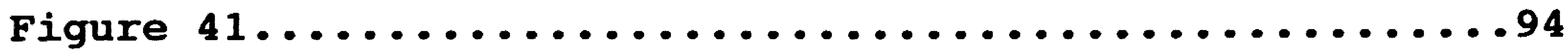

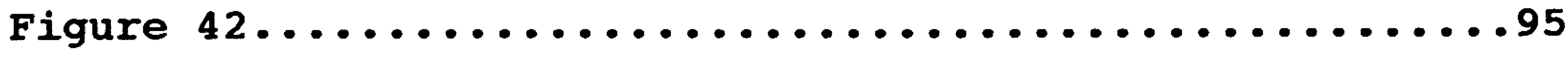

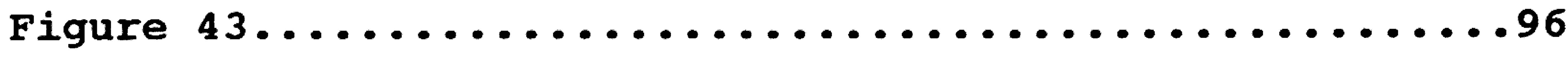

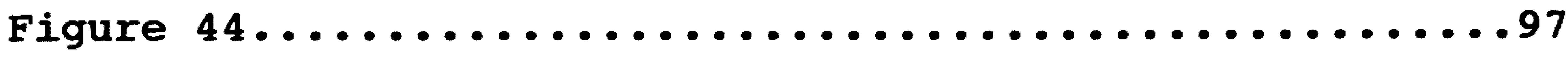

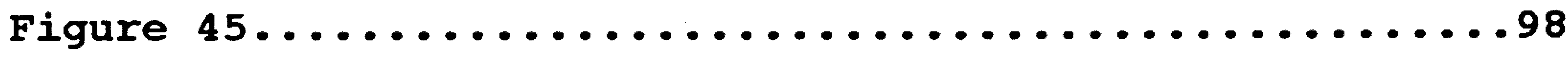

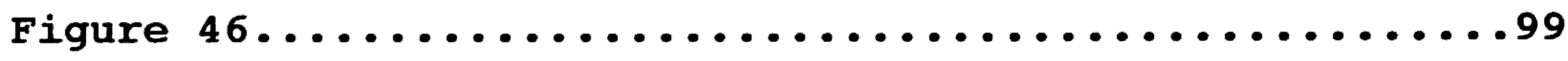

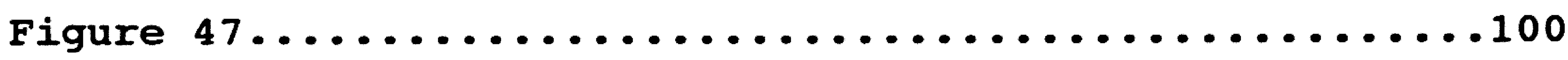

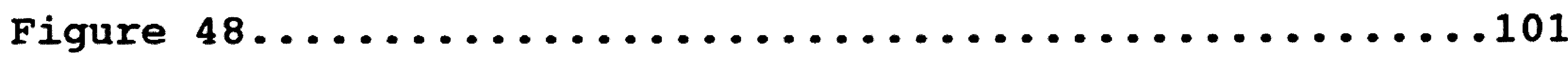

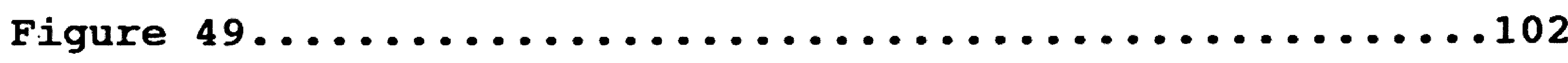




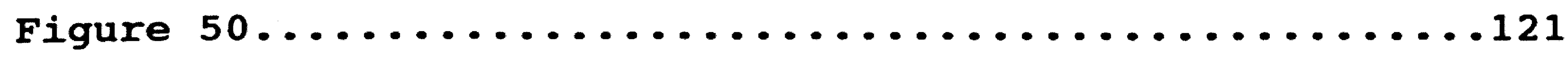

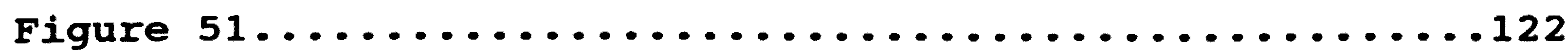

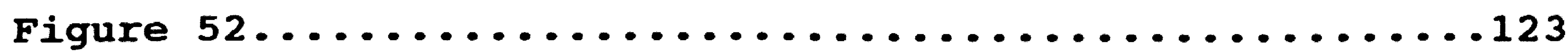

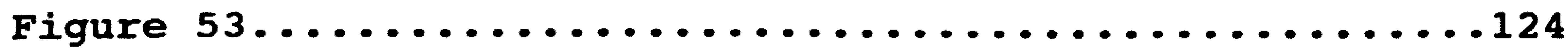

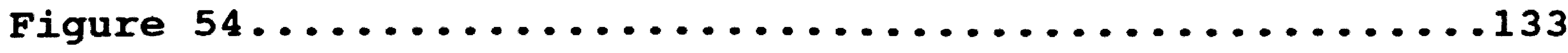

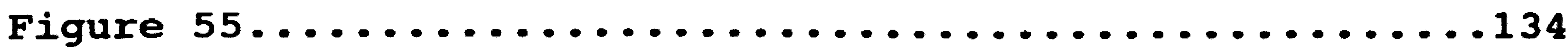

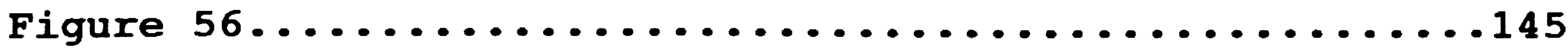

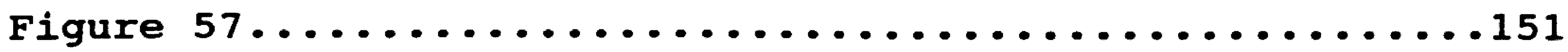

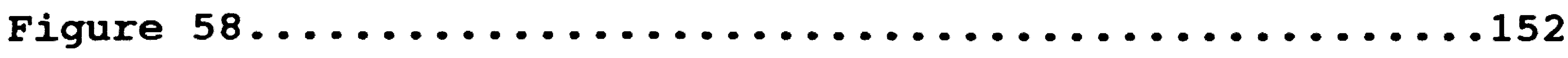

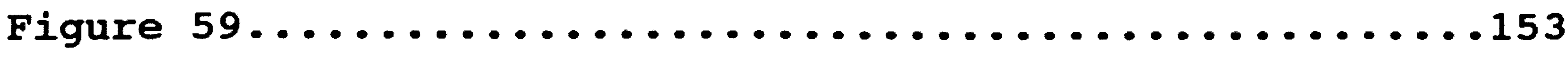

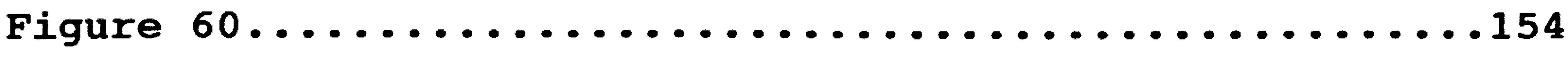

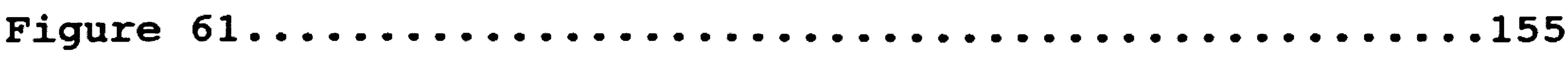

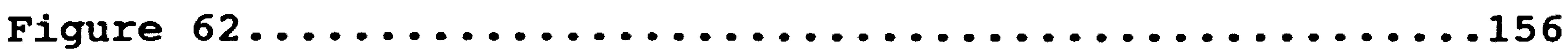

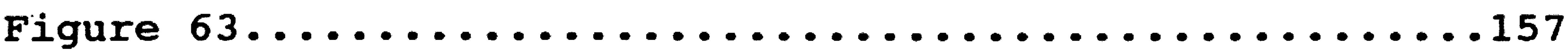


IIST OF TABIES

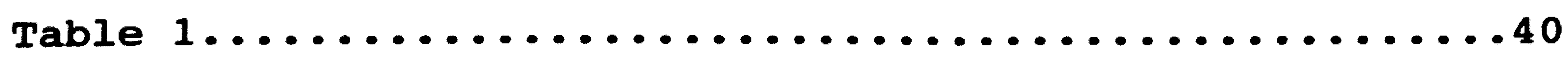

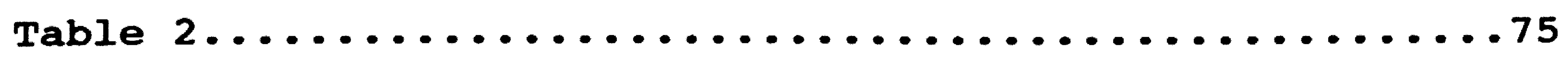

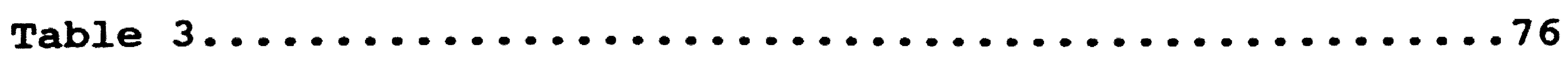

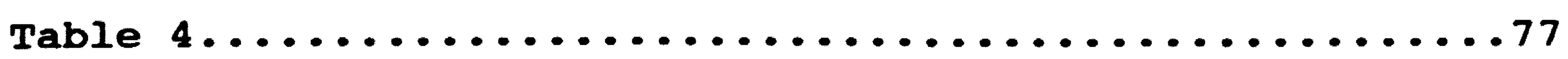

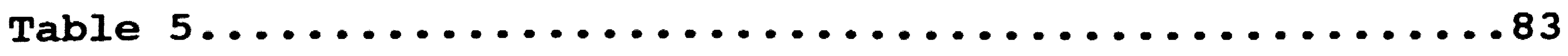




\section{INTMRODUCTION}

The aim of this study is the improvement of the present techniques used to assess the performance of natural and prosthetic heart valves. This improvement will be accomplished in two different sections: 1) A thorough study of currently used orifice area formulas, which includes the determination of the values for the coefficient of discharge of valves, as well as the scope of the formulas' applicability; 2) The derivation and proposal of a different method of assessing performance of prosthetic heart valves that is thought to be much more powerful than the currently popular method. This new method is the Energy Inder method.

The Effective Orifice Area (EOA), or opening area, of cardiac valves has been used as a measure of valvular performance. The EOA can be calculated from knowledge of the blood flow rate, pressure drop across the valve and its discharge coefficient. This formulation is attributed to Gorlin and Gorlin [1], but other investigators followed with corrections and modifications to the original formula. The formulas of Aaslid [23] and Gabay [24], as well as Gorlin's, will be used in this study.

Even though the Gorlin equation has been an accepted method of assessing valvular performance since the 1950 's, its predictive ability is still questionable due to difficulties 
associated with the determination of the coefficient of discharge, specially that associated with the mitral valve and its pathologies. Thus, in the first part of this study the author experimentally determines coefficients of discharge for three types of mitral valve prosthesis while subjected to a variety of flow conditions and pathological states.

Since the four orifice area formulas used were found to be inadequate for proper assessment of valvular function, the author derives the onergy inder method, which gives the physician faced with the prospect of electing a valvular prosthesis for implantation a single, quantitative, measure of the valve's performance that is self-correcting for valve size and type. The power index method finally solves the problem of assessing performance with a method that takes into account not only the forward flow characteristics of the valve, but also incorporates the regurgitant and leaking volumes through the valve while still using just one numerical parameter. 


\section{Chapter 1: PEISIOLOGICAT ASPECTS OF REIFUANCE}

The Euman Cesdiorasculax Bystam

The cardiovascular system is comprised of several components: the heart, the lungs, and the arterio-venous (or vascular) system. A description of the hemodynamic circuit that is seen by the blood as it circulates through the body could begin at the heart. The heart is a double pump, which means that it has two different sides, with similar functions, operating in series. The right side is responsible for what is called the pulmonary circulation (blood flow through the lungs), while its left side is responsible for the systemic circulation (blood flow through the body).

Venous blood, which is rich in carbon dioxide, collects in the right atrium through the superior and inferior vona cava. The vena cava are two major veins that collect the blood from the head and arms, in the case of the superior, and from the abdomen and legs in the case of the inferior. This blood enters the right ventricle because it still possesses enough head, or pressure - imparted onto it by the previous pumping cycle - to flow in that direction. The right atrium is separated from the right ventricle by a uni-directional valve, the tricuspid valve.

Once the right ventricle has relaxed from the previous 
contraction, the pressure inside of it drops considerably bellow that at the atrium, causing the tricuspid valve to open and a new charge of blood to enter the ventricle. When filled to capacity, the right ventricle starts to contract; as a result, the tricuspid valve closes passively and the pulmonary valve opens, sending that volume of blood to the lungs for oxygenation.

The pulmonary veins are responsible for collecting the blood from the lungs and delivering it to the left atrium. It should be noted here that the pulmonary veins represent an exception in the body in that, all other veins convey oxygendepleted blood, whereas the pulmonary veins convey oxygen-rich blood back to the heart.

The left atrium is separated from the left ventricle by the mitral valve. When the left ventricle relaxes, its internal pressure drops bellow that at the left atrium. As a result, the mitral valve opens and the blood fills the left ventricle. Upon contraction of the muscle, the mitral valve closes and the aortic valve now opens, forcing high-pressure, oxygenated blood to circulate throughout the body, exiting the heart through the aorta. Even though the contraction of the right and left sides of heart were described separately here, in reality, they happen simultaneously.

At the outlet of the aortic valve the inlet for a very important set of arteries is found: The coronaries, of which there are two main branches; so important are the coronaries 
(from the latin 'crown') in fact, that coronary circulation is usually treated as a subject in itself in medical physiology • The coronary arteries are responsible for keeping the heart muscle itself alive; it is the obstruction of the coronaries that leads to heart failure, known as infarction. Blood flow into the coronary arteries is facilitated by a special anatomic feature of the ascending aortic arch: the sinuses of Valsalva. The coronary arterial walls have to be compliant, for they must move with the myocardium at every stroke. At the same time, that compliance causes said arteries to collapse during systolic contraction (at least the smaller branches), for the myocardial wall is under circumferential tension and radial compression. A collapsed artery could only convey blood at a higher pressure than that which caused it to collapse in the first place, something that would only be logically possible if there were a separate pressure sourcel Nature's solution to this hydraulic problem was to place three small bulging pods circumferentially distributed around the ascending aortic arch, just downstream of the aortic valve. In fact, the cusps of the aortic arch partially cover these 'pods', which are called the sinuses of Valsalva, while the valve is open during systole. This intricate geometry promotes the formation of a rotational flow pattern within the sinuses with consequent accumulation of blood which flows into the coronary arteries upon ventricular relaxation. Figure one depicts schematically the source of coronary circulation. 


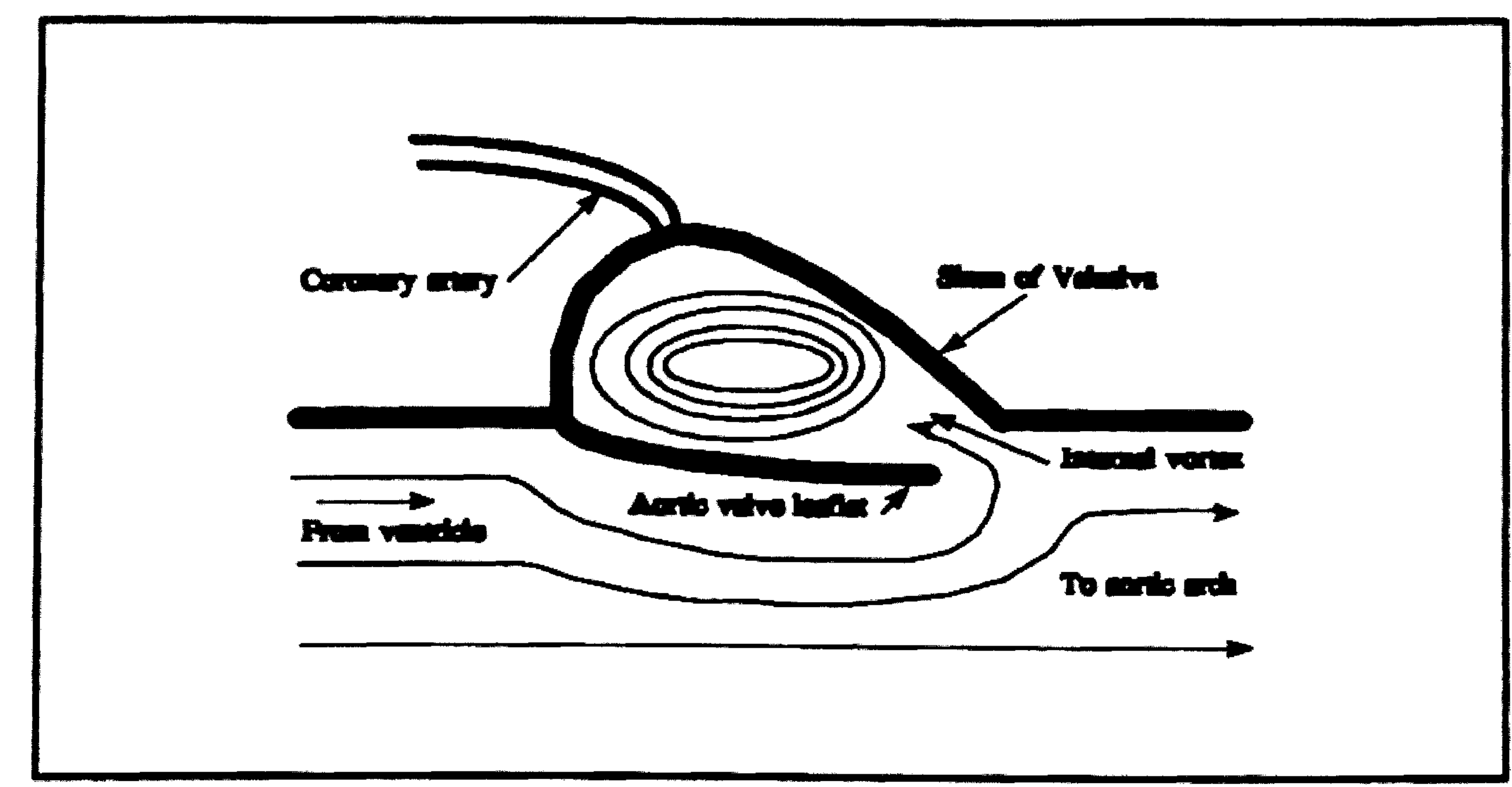

Figure 1: schematic representation of blood flow within the sinus of Valsalva. Blood enters coronary artery upon ventricular relaxation.

The distribution of blood to all cells of the body starts at the aortic arch, and, being divided into arteries anc arterioles, ends at the capillaries, where all molecular exchanges by the blood take place. The blood again collects, from the capillaries, into the venule and veins. Eventually, the oxygen-depleted blood reaches the two vena cava and enters the right atrium. The aortic pressure must be sufficiently high to ensure that the pressure at the vena cava is higher than that at the right atrium, even after being lowered through the resistance that the body represents, otherwise the flow of blood would be impeded. It is at the arteriole level that most of the distributional control of blood happens. Sections of the body which can forego optimal irrigation for short periods of time, in order to provide extra blood to other areas, have the arterioles that feed them constrict, 
thus decreasing blood flow to that region.

Once at the right atrium, the blood is ready for a new cycle identical to the one described above. This cycle will take place, on the average, 86400 times in one day, 2.5 billion times in a lifetime. Figure two depicts the various pathways of the human circulatory system.

Figure 2: Schematic diagram showing various routes of circulation. Figure adapted from Myers [10].

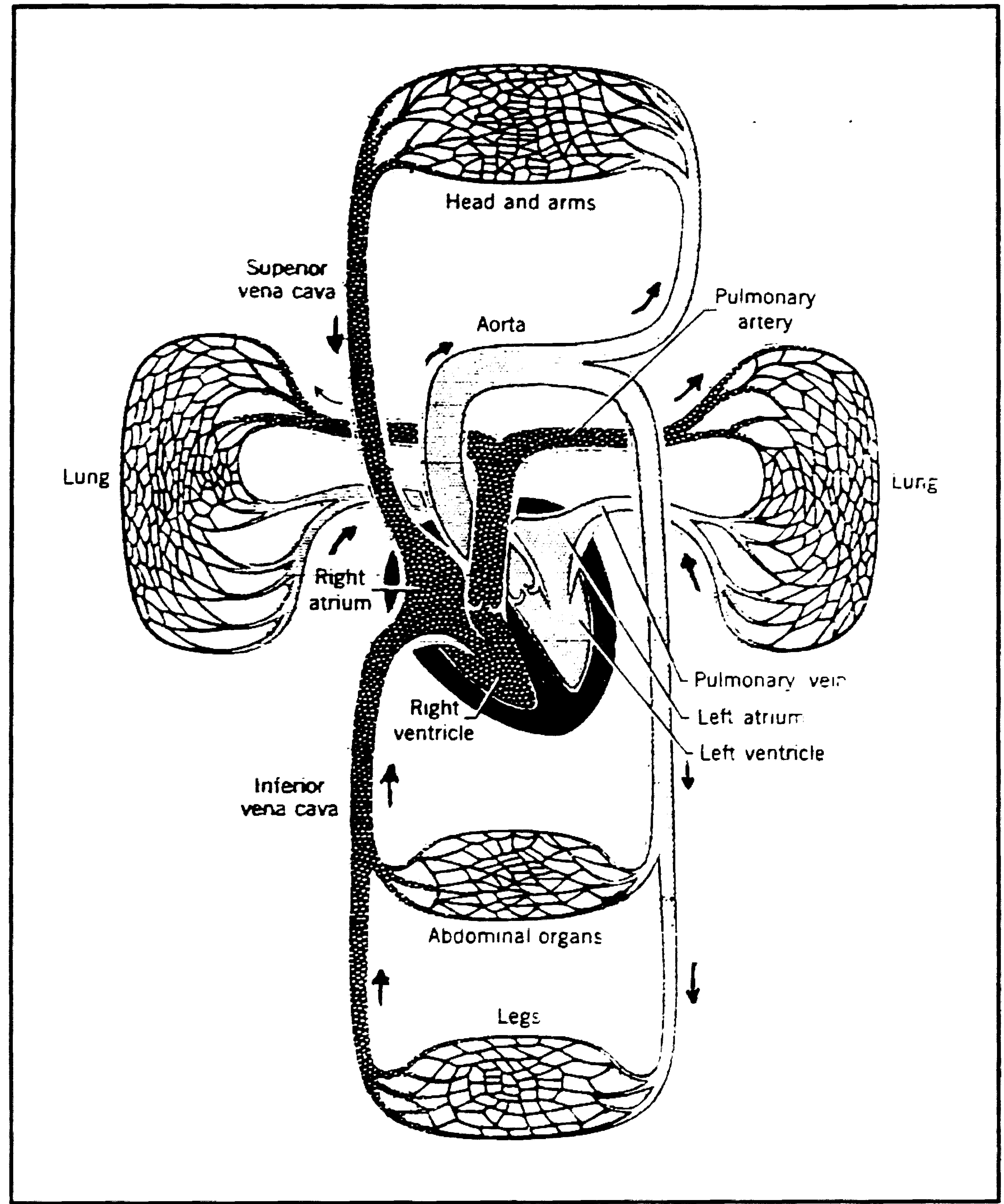


viewed externally, the heart seems to be a continuous mass of muscle tissue, but, in reality, there are two auricles - or atria - and two ventricles. The two ventricles are separated from each other by a thick wall called the soptum, and from their corresponding auricles by a valve. From the description of the circulatory system presented earlier, it seems apparent that these four chambers cannot contract - and thus eject their charge of blood - at the same time. There is a very delicate synchrony and sequence of contraction within the heart muscle which accounts for its function. Examination of the muscular contractile process will yield insight into the operation of the heart.

Muscle fibers, such as those out of which the myocardium is composed, have the ability to contract when reached by a suitable electrical signal termed action potential (AP). Since each cell is able to contract, independently of its neighboring cells, once reached by the proper electrical signal, the secret to controlling the heart lies in controlling the distribution of the action potential to each cell of the muscle.

External electrical control reaches the heart at a location termed sinoatrial node (SA node) through the vagus nerves. This is also the point at which the action potential is initiated if, for some reason, no external stimuli is 
present. This 'self-sufficient' operation is possible for humans and other mammals that are provided with myogenic hearts. From the SA node, the AP propagates from one atrial cell to another, one at a time, until finally reaching a location termed atriovontricular node (AV node). The AV node delivers its charge not to its neighboring cells, but to a thick nerve called bundle of His, which splits into two main fibers called the left main bundle and the right main bundle. The two main bundles terminate into what is known as the Purkinje fibers. The Purkinje fibers are much finer and more numerous than the main bundles, thus they are able to reach a great number of cells at the same time. The sequence of fibers described above bring the AP all the way to the apex of the heart before delivering it to the muscle cells, thus making it possible for the heart to contract from the apex up, towards the pulmonary and aortic valves.

The tissue that lines the internal section of the heart muscle, the myocardium, is called endocardium. The endocardium is a special tissue with some outstanding wear and frictional properties. The entire heart, as an organ, is enveloped by a tissue called pericardium. The pericardium is a somewhat translucent tissue that is liquid filled and has the function of protecting the heart, not only from thermo-mechanical shocks, but also from biological hazards.

The heart valves, being uni-directional, are responsible for keeping the blood moving in one direction upon ventricular 
contraction. Blood is admitted into, and ejected out of, the left ventricle by the mitral and aortic valves respectively. During ejection the mitral valve is closed. Pressure drop across an ideal valve is negligible while open; its closure should take place with negligible flow.

The atria, at the inlets of the ventricles, accumulating the blood that would otherwise take a longer time to fill the ventricles, couple the nearly steady flow at the vena cava to the highly pulsatile flow into the ventricle.

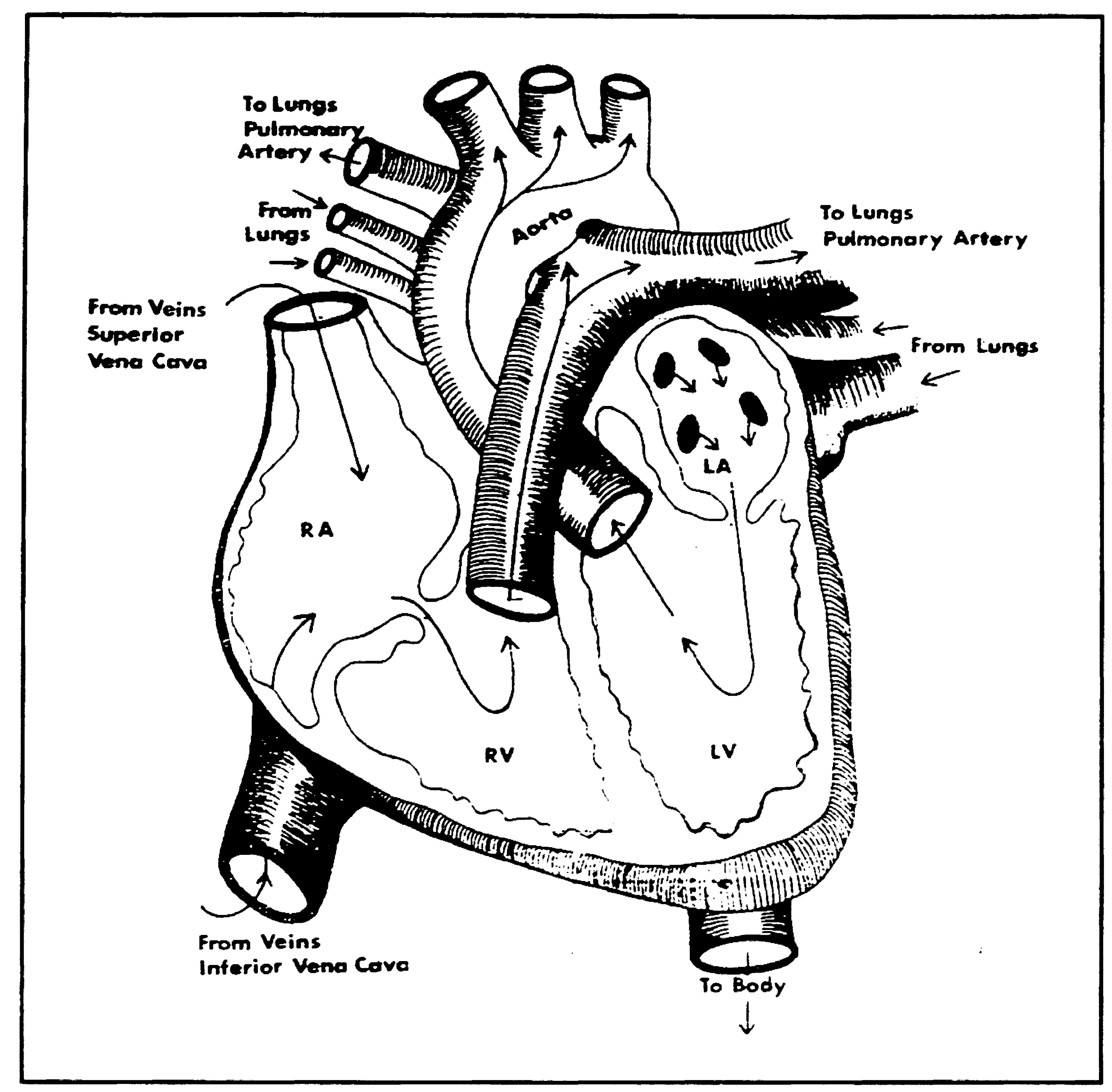

Figure 3: simplified internal diagram of the heart showing its four chambers and circulation routes. From Myers [10] 
All four of the valves found in the heart, two between the ventricles and the atria and two at the outlets of the ventricles, are of a similar nature. The mitral valve, being the object of this study, will be briefly described.

The mitral valve is a complex unit that has to be analyzed and understood in conjunction with six major components: 1)the left atrium, 2)the mitral annulus, 3)the mitral leaflets, 4)the chordae tendineae, 5) the papillary muscles, 6)the left ventricular myocardium. The mitral valve has two thin leaflets, or cusps, that flap up and down, their ends coming together when the valve is closed. Each of the two leaflets are connected to inner walls of the myocardium through a set of tendons called chordae tendineae and the papillary muscles. These muscles and tendons prevent the leaflets of the mitral valve from folding back into the atrium once the valve is fully closed, under systolic pressure. Under these conditions, the leaflet is supported at the root by the ventricular wall, and at the tip by the tendons. The leaflets themselves, being very thin, require some stiffening; a set of ridges, made in the same material as the leaflets themselves does just that. These ridges, much like beams on a plate, are called commissures. The commissures play another important role in the function of the valve: since the leaflets are not hinged at their roots, the leaflet has to bend in order for 
the valve to open and close properly; the stiffness of the commissures must be sufficient to withstand systolic pressure, but low enough for effective opening during diastole.

Common Malfunations

Malfunctions, or pathologies, of any of the four valves found in the heart can be divided into two groups: stenosis and insufficiency.

A stenotic valve is one that does not open fully, usually because of stiffening or fusion of the valve leaflets in the commissural area. Stenosis of the valve causes smaller than optimum opening areas, which in turn will cause high transvalvular pressure gradients. One consequence of stenosis of the heart valve is a lower cardiac output, which may cause damage to the body due to the lower oxygenation of tissues. Stimulated by oxygen sensors located throughout the body, the myocardium will contract ever more strongly, in an attempt to compensate for the stenotic valve.

If the stenotic valve is in the mitral position the higher than normal pressure gradient across it will preclude the ventricle from being filled to capacity, even with increased atrial activity. As explained by the Frank-starling law, a partially filled ventricle will contract with a lower force because its muscle fibers are less stretched. The ventricle will eject a lower volume of blood per stroke at 
lower pressure, i.e. a lower cardiac output. Normal right ventricular output, coupled with a stenotic mitral valve may result in accumulation of blood in the porous tissue of the lungs, to the point of rupture. Patients that fit this clinical scenario have coughed large amounts of blood.

If the stenotic valve is mounted at the aortic position, the ventricle can still be filled to capacity, but it probably will not because of the lower cardiac output of the previous cycle (scavenger effect). A ventricle that is ejecting blood through a stenotic valve will not deliver the same volume per stroke, at the same pressure, as a normally valved ventricle. A lower output pressure, dissipated by the same systemic resistance will yield a lower atrial input, describing an unstable system because lower atrial flow will cause lower ventricular filling. Lastly, stenosis of the heart valves could be caused by rheumatic fever or congenital defects.

The other common malfunction of heart valves is insufficiency. An insufficient valve is one that cannot close completely during systole (if it is the tricuspid or mitral valve), or during diastole, (if it is the pulmonary or aortic valve) allowing blood to be regurgitated back into the atria or ventricle respectively. Insufficiency is most often associated with the mitral valve; because high ventricular pressures occur during systole, a period that finds the aortic valve open, but the mitral valve closed in resistance to that pressure. 
It is the function of the chordae tendineae and that of the papillary muscles to prevent the leaflets of the valves from folding back in to the atria, which would allow regurgitation of blood, as explained earlier. Rupture of the chordae or loss of muscular contraction (Papillary) could account for the sort of insufficiency in which the leaflets fold back into the atria. The other possible mechanical cause of insufficiency is, again, the stiffening of the leaflets. Finally, insufficiency may be the result of a congenital defect, a diseased state, or even the result of a surgical complication.

Heart valves can be substituted by prosthetic devices. Artificial heart valves have been used to replace diseased ones ever since the mid-1960's, soon after the open heart surgery was a reality. The replacement of heart valves usually enhances the life of the patient; reportedly, 708 of patients have survived for five years or longer after the surgery - [12] The biggest problem associated with prosthetic devices continues to be thromboombolism, damage to the blood due to contact with foreign materials. Thromboembolism is caused by bioincompatibility and adverse dynamic characteristics of prosthetic devices [25]. More recently, blood cavitation at the valve occluder has been suggested to cause hemolyses. High occluder to housing impact velocities at closing and the retrograde jet of blood through the valve, while it is closed, were suggested as possible causes of cavitation. Improvement 
of heart valves, then, as in all other prosthetic devices, must come in the area of materials, biocompatibility, and hemodynamic design. A prosthesis should not cause undue pressure gradients or high shear rates in the flow field. As seen, then, while the actual design of a prosthetic heart valve would include the areas of materials, durability and performance, the present study is aimed solely at the dynamic performance assessment.

Prosthetic valves can be totally mechanical or made from biological tissue, such as a bovine pericardium or a porcine aortic valve. While tissue valves present a higher degree of biocompatibility, generally they do not last, mechanically, as long as the purely mechanical valves do.

The three most common types of mechanical heart valves in use today are the ball and cage, tilting disc, and tho bileaflet. Of those, the oldest is the ball and cage and the most recent design is the bileaflet. The ball valve shows very little regurgitation, but it is also damaging to the blood because of the turbulence it creates. Many models and brands of each design, as well as other designs, are available on the market. [10] 
Having established what the most important problems associated with mitral valve are, an explanation of the techniques used for their detection is in order.

The presence of valvular regurgitation can be detected by phonographic means because the retrograde jet of fluid created causes a distinct acoustic wave to be generated. Optionally, Doppler-echocardiography can produce excellent representations of the flow pattern, both in the positive and in the retrograde direction of the valve. These techniques are, at best, qualitative, however. Most recently, three-dimensional reconstruction of the planar echocardiographic record has been shown to report regurgitance with more precision.

Stenoticity can be detected and quantified with orifice area formulas. In this study, the four most important orifice area formulas are described and used.

Assessing the condition of the valve with an orifice formula requires the use of the correct value for the coefficient of discharge, the determination of which is one of the main topics of this work.

Having confined the scope of what can be learned about the function of heart valves by the use of orifice area methods, a description of what has been accomplished by previous investigators is in order.

The first to introduce an orifice area equation for the 
purpose of valvular function assessment, was Gorlin [1] in 1951. The Gorlin equation, derived from laws of conservation of mass and energy through a discharge orifice, requires the knowledge of the mean pressure drop across, and the mean flow rate through, the valve, as well as its coefficient of discharge. The first two quantities can be measured in vivo and in vitro, whereas the coefficient of discharge must be determined empirically.

In 1975, Aaslid and associates [23], proposed an equation similar to that of Gorlin for valvular function assessment. Aaslid's method, though, uses the peak flow rate through the valve and the pressure drop at the time of peak flow. It is in order to alleviate the effects of acceleration of the flow through the valve that Aaslid uses the peak flow rate, instead of the mean. In 1978 Gabay [24] introduced another equation for valvular function assessment; the Gabay method uses the RMS flow rate and the mean pressure drop. As is the case with the other two equations, the Gabay method also demands knowledge of the coefficient of discharge.

Because it uses the mean flow, which can be obtained clinically, the Gorlin formula remains the most popular tool for function assessment of heart valves. Studies have shown, however [24], that large errors can be incurred when the Gorlin formula is used. Swanson [18] demonstrated that the pressure drop across prosthetic heart valves is proportional to the flow rate exponentiated to the 1.5 to 2.0 power; 
dimensional requirements, however, demand that the formulas described above use the pressure drop being proportional to the flow rate exponentiated to the 0.5 power. Finally, Schoephoerster [15], showed that it is appropriate to use a coefficient of discharge, with the orifice formulas described, which itself, is a function of the flow rate through the valve. This determination was made theoretically and validated with in vitro studies.

since all of the investigators mentioned, including Gorlin who only studied natural valves, showed that different geometries of valve demand the use of different values for the coefficient of discharge, and since this value has also been shown to vary with flow rate, before valvular function assessment can be carried out, one is justified in asking whether the value for the coefficient of discharge may also vary with valvular condition, which it is supposedly determining. Clearly, should this be the case, the orifice area method cannot be said to work; for, in trying to learn about a heart valve's condition, it is impossible to know what that condition is a priori, as would be the case with a coefficient of discharge that changes with different degrees of stenoticity. 
Formulation of the Problem and Mothod Solution

The equation derived from conservation of mass and energy laws of fluid mechanics by Gorlin \& Gorlin [1], known simply as the Gorlin equation, has been used for many years as a means of predicting the effective orifice area, (EOA) or the effective opening area of a natural or prosthetic heart valve. The EOA is a useful clinical tool of assessment of valvular performance because its value can be compared to a known valve area, discrepancy with which indicates suboptimal operation.

$$
E O A=\frac{Q_{\text {moon }}}{44.5 \times C_{d} \times \sqrt{\Delta P_{\text {mean }}}}
$$

Where: $Q=$ Mean flow rate of blood while value is open: $\mathrm{cm}^{3} / \mathrm{sec}$.

$C_{d}=$ Discharge coefficient

$\Delta P=$ Mean pressure drop across valve: $\mathbf{m m H g}$.

44.5 is a constant that includes gravity.

Application of the above equation to the analysis of heart valves followed observation that the valvular annuli of diseased heart valves remain essentially fixed (their geometry does not deviate from that of a central-flow orifice and they do not translate or rotate relative to the surface they are mounted on) during a cardiac cycle. In their 1951 paper, Gorlin and Gorlin [1], state that: 
Because of the essentially fixed nature of the rings of the cardiac valves when diseased, an attempt has been made to apply the hydraulic principles and formulas of fixed orifices to these stenotic valves.

Because the mass of a flowing fluid within a conduit must be conserved, the flow rate (or discharge) of the fluid will be numerically equal to the cross sectional area of the conduit multiplied by the velocity of flow. If that flow is made to cross an orifice, it will be seen that the resulting cross sectional area of the fluid in motion, just downstream of the orifice, will be somewhat smaller than that of the orifice itself. The equality suggested above can be preserved if its right side is multiplied by a constant termed coefficient of contraction, a function of the geometry of the orifice.

$$
Q=C_{c} \times A \times V
$$

where: $Q$ is the flow rate: $\mathrm{cm}^{3}$ per second.

$C_{c}$ is the coefficient of contraction.

$A$ is the area: $\mathrm{cm}^{2}$.

$V$ is the flow velocity: $\mathrm{cm}$ per second

The auricle and ventricle being the conduit, interrupted by the valve, or orifice (when open), is a feasible model to which the equation of conservation of mass can be applied. similarly, using the same model, it is possible to apply the 
principle of conservation of energy across the valve. The fluid that is above the valve (orifice), but not moving, has potential energy, while the fluid that already went through the valve, now filling the ventricle has less potential, but some kinetic, energy. The difference in potential energy of the fluids before and after the orifice is numerically equated to the kinetic energy acquired though its motion by the coefficient of velocity, a function of the fluid's viscosity.

$$
V=C_{v} \times \sqrt{2 \times g \times h}
$$

where. $V$ is the velocity: $\mathrm{cm}^{2}$ per second.

$$
C_{v} \text { is the coefficient of contraction. }
$$

$g$ is the gravitational acceleration: $\mathrm{cm}$ per second ${ }^{2}$. $h$ is the fluid head: $\mathrm{cm}$.

The last two equations presented can be combined to yield the Gorlin equation. The constant 44.5 in the Gorlin formula reflects the square root of the quantity $2 \times g$, in centimeters per second per second. The transvalvular pressure drop should be used in centimeters of fluid and the flow rate in cubic centimeters per second.

In order to use the Gorlin equation as a mean of assessing the performance of a heart valve, pressure at each of its sides is acquired during several heart cycles. Such measurement is easily accomplished by means of cathetermounted pressure sensors and fluoroscopy • 
A small incision in the groin or shoulder area is needed for the insertion of a single catheter that looks much like a flexible plastic wire, about $1 / 16^{\prime \prime}$ in diameter. The catheter can have two pressure sensors attached to its end, about 1 inch apart axially. The position of the incision depends on the specific valve that is under investigation: if the mitral, the incision will be in the groin; if the aortic, the shoulder area.

The patient is placed on a fluoroscopic table, such that the position of the tip of the catheter is visible on a screen at all times. With much skill, the tip of the catheter will eventually reach the heart via the incision described. Because the soft tissue of the valves, as well as the catheter, can be seen on the screen, the experimenter can wait until the valve opens and then insert the catheter another inch or so, such that its tip, with one of the pressure sensors, is now inside of the ventricle. Pressures on both sides of the valve can now be recorded.

Blood flow rate across the valve can be estimated by the Fick method of oxygen consumption or die-dilution. [17] These techniques, traditionally used, can only produce mean flow rates; thus the mean flow and the mean pressure drop were used originally to compute EOA. Both of the pressures as well as the flow rate will be cyclic, rather than constant quantities, which suggests that the quantities have to be averaged over many cardiac strokes (typically more than 15) in order to 
obtain values that are representative of the operation of a valve. With increased in vitro testing of mechanical heart valves, and the advent of sophisticated measuring equipment, attention was redirected to the Gorlin formula in search of possible improvements. The most noteworthy contributions were those of Aaslid [23] and Gabbay [24], who suggest the use of the peak instantaneous flow rate and the pressure drop at the time of peak flow, and the use of the Root Mean Square of the flow rate and the mean pressure drop, respectively. The constant 0.32 in the Aaslid equation arises because of conversion of units; while the Gorlin and the Gabay equations use 'ml/sec' for flow and centimeters of fluid for pressure drop, the Aaslid equation uses 'liter/min' and 'mm Hg' for flow and pressure drop respectively.

The Aaslid equation:

$$
E O A=0.32 \times \frac{Q_{p}}{\sqrt{\Delta P_{Q P}}}
$$

The Gabay equation:

$$
E O A=\frac{Q_{\text {RMS }}}{44.5 \times C_{d} \times \sqrt{\Delta P_{\text {mean }}}}
$$

All three equations use the same simplifying assumptions, namely: flat velocity profile upstream of valve; central-flow, circular orifice for valve configuration; static fluid 
upstream of valve; inviscid fluid and ideal orifice geometry • In order to account for these assumptions, all three equations use an all-encompassing constant to correct the calculated orifice area. Gorlin's coefficient of discharge is determined empirically by solving the Gorlin equation and dividing it by the mosured open valvular area. Aaslid's effective area index is the quotient of his formula by the mounting area of the valve. Gabay divides his formula by the primary flow area of the valve, deriving the coefficient of performance, or the mounting area, deriving the performance index.

Although the Aaslid and Gabbay formulas have been shown to be superior in area prediction to the Gorlin formula [24], the latter remains the most popular clinically because the estimation of mean flow rates does not require the use of sophisticated equipment.

Since the values of the correction constants described above directly affect the calculated effective area of the valve, and since this area is used to diagnose the condition of the valve, precise determination of the values of the constants and the conditions for which these values are valid are of utmost importance, and represent, in fact, one major objective of this study.

The dependence of the discharge coefficient on the flow rate for a porcine valve mounted in the aortic position has already been established by schoephoerster et al [14].

In order to actually measure what the discharge 
coefficient is for the mitral valve operating under any set of conditions, a cardiovascular duplicator, that puts the valve in a flow and pressure field similar to physiological, is built and used to measure flow through and pressure drop across three different types of prosthetic heart valves, operating at three degrees of stenoticity each. Two sizes of each valve and thirteen flow rates are used in each case, yielding 234 measurements designed to confirm the Cd's variance with Reynold's number, and study its possible variance with valve type, size and condition of stenoticity. Actual opening areas are measured by photography and planimetry. Details of the experiment are described later.

Erperimental Apparatus

The equipment designed and built at Florida International University is termed a Cardiovascular Duplicator. The function of a cardiovascular duplicator (CVD) is to provide the heart valve, the object of the study, - whether natural or prosthetic - with an operating environment as similar to the physiological one as possible. The CVD is composed of five independent systems: The pulse duplicator (PD), the cardiac omulator (CE), the systomic simulator (SS), the ventricular system (VS) and the data acquisition sYstem (DA).

The pulse duplicator is the module responsible for producing flow and pressure waves in forms that approximate 
those produced by the human heart, as published by Archer [19] and Weissler [20]. It consists of a piston and cylinder arrangement such that hydraulic fluid is pumped in and out of the pericardial box of the cardiac emulator. Figure four depicts selected details of the pulse duplicator.

The piston is driven by a stepping motor, through a rack and pinion, such that its position and velocity can be accurately controlled. A personal computer transforms the desired flow and pressure wave forms into a $v(t)$ signal for the motor by means of a program written by the author. The signal from the PC is then fed to an MC68HC11-based pulse generator, then to a motor controller, which also receives input from an external power supply, and is finally fed to the motor. The mechanism includes three limit-switches; one to determine a 'home' position, from which the stroke length is measured, and two others, one at each end of the available travel of the rack to turn the motor off in case an error causes the latter to run beyond the length of the former. The PD, as a unit, contains an internal power supply to run the logic boards and a fan to cool the motor and motor control board. The whole unit is enclosed by a sound-proof box which contains an air cleaner, the various connectors, a home-stop button, and a fuse holder. The home-stop button, if pressed, causes the motor to stop at the 'home', with the ventricular sac in its full-diastole position. This feature is important to determine the end-diastolic ventricular volume and shape. 


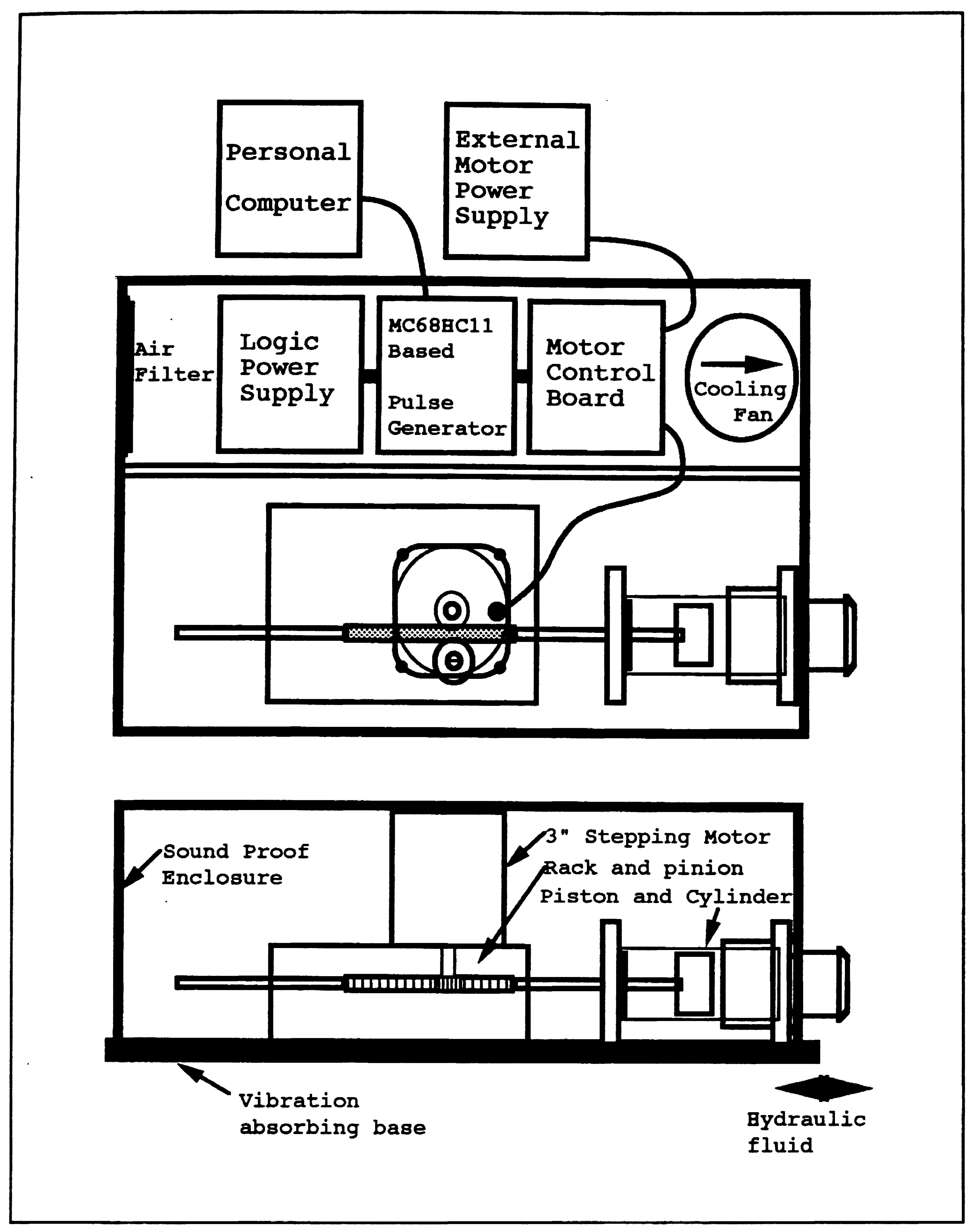

Figure 4: Selected details of the Pulse Duplicator 
The cardiac omulator places the heart valves in a suitable flow and pressure field because of its unique design, which incorporates a contractile ventricular sac and a ventricular load with unique inertance, capacitance and resistance characteristics.

A flexible model ventricle was cast out of flexible silicone rubber. This ventricle is placed inside of a rigid box, in and out of which the pulse duplicator pumps hydraulic fluid. Since the model ventricle is flexible, and the pericardial box is rigid and completely filled with an incompressible fluid (50\% Glycerin, 50 distilled water), there will be a positive correspondence between the position, velocity and acceleration of the piston and the contraction of the ventricle.

At the top of the cardiac emulator the aortic and mitral valves are mounted. The geometry of the relevant parts mimics the physiological ones as closely as feasible, following recommendations reported by Duplessis, et al. [21] Close consideration of the need to frequently change heart valves and open the $C E$, as well as the possibility to visualize and photograph the various flow fields, resulted in a versatile design that can be completely disassembled in minutes and photographed from any angle.

The systemic simulator duplicates the complex load against which the heart pumps, for, while the transvalvular flows are solely determined by the pulse duplicator, (a 
function of the velocity of its piston) the pressures developed in the system are a function of the load the ventricle pumps against. As explained in the background section of this paper, the heart needs to impart a pressure increase, and thus flow, to the blood. That pressure increase (Potential) is transformed into flow, but not totally, for some of the pressure is lost due to frictional and form (or hydraulic) resistance. It is vital then for a CVD to duplicate the systemic resistance the heart must pump against. The physiological range of magnitudes usually found in the systemic circulation vary from 0.58 to $1.13 \mathrm{~mm} \mathrm{Hg} . \mathrm{s} / \mathrm{cc}$. (Millimeter of Mercury times second divided by cubic centimeter; prossure drop over flow rate).[6] Systemic resistance must not only be simulated in the CVD, but its adjustment also provided for, such that flow rates can be varied without the need for the development of nonphysiological pressures. This process in the human body is called vaso-constriction. In the CVD resistance is created by running the fluid through a tube bank. The characteristic resistance of the $\mathrm{CV}$ system is duplicated by 950 tubes, 1.5 inches in length, glued in parallel and located just distal (downstream) of the characteristic compliance chamber. The peripheral resistance was duplicated by 250 tubes in parallel, 4.75 inches in length, but this time mounted in such a way that a rotary gate allows occlusion of none to all of the tubes; thus going from a minimum resistance value to total 
occlusion of the flow. These two resistors were designed to be linear; in other words, a linear pressure drop with increased flow rate through the tube bank is desired. Design of the resistive elements followed recommendations by Westerhof et al. [32]

The blood that will be distributed through the body is conveyed out of the heart by the largest artery found in the body: the aorta. The initial segment of the aorta is called the aortic arch, whose compliance is critical. That elasticity, or compliance, has to be reproduced by the CV Duplicator, its value should also be adjustable to accommodate any irregularities of the system, but the nominal physiological value typically found is $1.33 \mathrm{cc} / \mathrm{mmHg}$ (Cubic centimeters per millimeter of mercury). [6] The compliance of the aorta attenuates the pulsatile flow and affects the peak pressures in the system, as can be inferred from Laplace's law, which states that the pressure developed within a vessel is proportional to the circumferential tension, $T$, on its walls, and inversely proportional to the radius of the vessel, R. Thus:

$$
P=k \times \frac{T}{R}
$$

Where $k$ is a constant of proportionality

A lower tension will be developed on the wall of a more 
compliant aorta, and thus a lower peak pressure achieved. In the CVD, compliance is duplicated by a chamber that can be pressurized and initially filled to a variable extent. A separate pressure monitor is employed at this section of the duplicator to ensure repeatable adjustment of the compliance. As in the case of the resistance, however, it is not necessary to display the exact value of the compliance.

In addition to the aortic compliance, the cardiovascular bed, being composed of elastic arteries and veins, also has its own elasticity, which gives rise to what is termed peripheral compliance. The veins, in particular, can hold up to $65 \%$ of the total blood volume under certain conditions, which means that the veins, collectively, function as a reservoir. The function of a reservoir in a closed loop under pulsatile flow is of extreme importance: because a volume of fluid is accelerated and decelerated during each cardiac cycle, the pressures in the system are also a function of the volume of fluid under circulation, upstream of the reservoir. The dependence of pressure developed on circulating volume is a simple consequence of Newton's second law of motion: in order to impart the same acceleration on a larger mass of fluid, a larger force, or in this case pressure, is necessary. It is hereby seen that, at the higher flow rates, under pulsatile conditions, a smaller volume of circulating fluid is needed if the same pressures are to be produced within the machine. The FIU CVD does incorporate a peripheral compliance 
and a reservoir, all in the interest of producing physiological pressure and flow wave forms.

It is thus seen that the Systemic Simulator addresses all of the physiological features normally associated with the human body that are relevant to the fluid-mechanical environment in which the heart valves are expected to work in. [18] Figure five diagrammatically shows the cardiac emulator and the systemic simulator.

The ventricular mystom is not used in its entirety during experiments, nonetheless its construction consumed a considerable amount of effort. This system is composed of three ellipsoidal cavities and an insert that, together, form the molds used to cast the rubber ventricles. Three cavities are used in order to produce ventricles of three different wall thicknesses, in case that variable becomes relevant to the flow distribution across the valves. In addition to the expected alignment pins used with any mold, this system also employs a removable mold for a flange to be cast at the base of the ventricular sac, relief and ejection pins, and its own mechanical apparatus used to brake the mold once the material cures fully. The molds were manufactured in aluminum using primarily a numerically-controlled lathe. The internal volume of the finished part is $200 \mathrm{ml}$, a figure determined by the need to have $70 \mathrm{~mm}$ of diameter at the base (in order to accommodate both heart valves) and an aspect ratio of 1.12 (length/base) proportional that of a typical human heart. [27] 


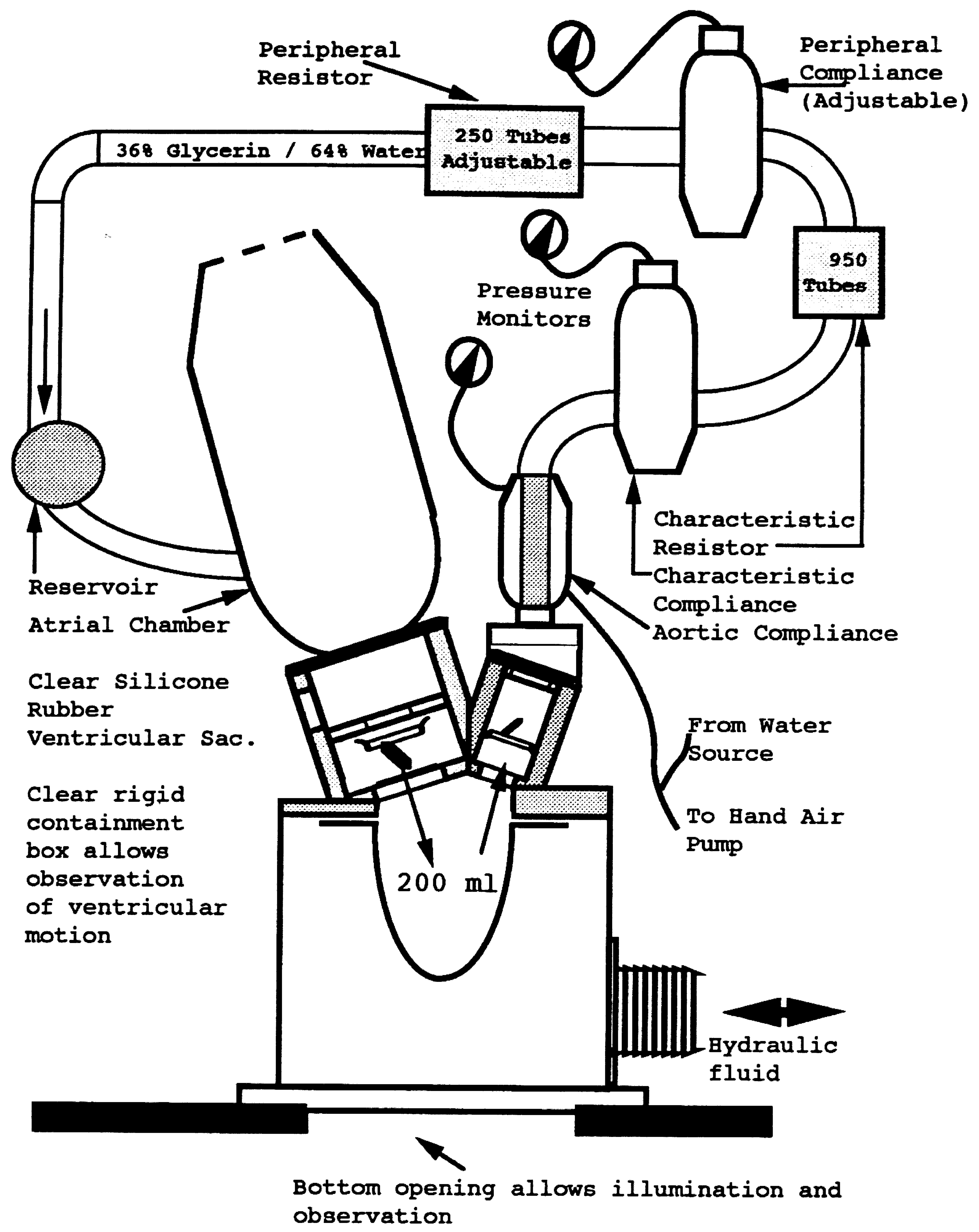

Figure 5: Selected details of Cardiac Emulator and systemic simulator. 
The data acquisition system is comprised of five transducers, four control units, a data acquisition board with an analogue-to-digital converter and a personal computer for data analysis and storage. Three disposable catheter pressure transducers (MPC-500) were employed in conjunction with their own, battery operated, control units (TCB-500) [17]. Battery operation of the control units was favored in an attempt to eliminate possible electro-magnetic interference with other electronic equipment operating simultaneously with the transducers. An electromagnetic flow probe, $50 \mathrm{~mm}$ in circumference, along with its control unit (501D) was used for flow measurements. [26] The fifth transducer employed consists of a switch, through which a 5 Volts signal is wired. Actuated by the rack of the PD every time it reaches 'home' (enddiastole), this switch causes a flag to appear in the data stream, indicating the separation between two consecutive strokes. The data acquisition board (WB 800) is capable of digitizing up to eight channels at a maximum rate of $20 \mathrm{Khz}$; it is mounted directly onto the computer's mother board [31]. The personal computer is an IBM compatible, sporting a 286class processor and a 287-class math co-processor, and a VGAclass color monitor. 
The determination of the flow wave forms that were used for function duplication was based on physiological information, which is summarized here. [20] [27] [28]

Before reading this explanation, the reader, especially those coming from an engineering background, is reminded that "normal" physiological measurements of any quantity vary from individual to individual. This analysis will then use values that are generally accepted to represent humans in general.

The resting adult heart contracts 65 times in a minute. While doing so, its left ventricle ejects 85 milliliters of blood. Under extreme exertion the same heart would contract 190 times in one minute, while its left ventricle would eject a volume 208 larger, $102 \mathrm{ml}$. [28]

Using the above points to describe a line, we can determine left-ventricular stroke volume (LVSV or SV since we are only dealing with the mitral valve and the left ventricle) for any cardiac frequency. With the SV and the heart rate (HR) we are able to compute the cardiac output (co) by direct multiplication of the former by the latter.

The last value missing to assemble the flow wave-form table is the duration of the systolic interval. The percentage of the cardiac cycle, systole plus diastole, (contraction and relaxation) that is occupied by systole has been noted to increase with increased heart rate. [20] 
Before explaining the pattern of systolic interval increase, it is necessary to describe the several phases of the systolic and diastolic period. When the action potential reaches the end of the many Purkinje fibers, the ventricular muscle cells depolarize and contraction of the myocardium follows. Before blood can be ejected, however, the ventricular pressure has to exceed that of the aorta, nominally $80 \mathrm{~mm}$ of Hg, (up from the nominal filling pressure of $5 \mathrm{~mm}$ of $\mathrm{Hg}$ ) hence a period termed isovolumetric contraction is observed, the first phase of the cardiac cycle. The second phase is called rapid ejection; here, up to two thirds of the stroke volume will pass through the aorta. The third period is called reduced ejection, during which the blood flow experiences deceleration and the aortic pressure may actually exceed slightly the ventricular pressure, in which case forward blood flow is maintained by the inertia of the fluid in motion. The ventricle then enters the fourth phase of the cardiac cycle in which isovolumetric relaration takes place. At this point, the ventricular pressure is decreasing rapidly, and when it is lower than that at the atrium, diastole begins with a period called rapid inflow, followed by reduced inflow, or Diastasis. The reason for diastasis is simple: as the ventricle fills, the atrio-ventricular pressure gradient decreases, consequently decelerating the flow through the mitral valve. Just before complete halt of the diastolic flow is reached, the Atrium actually contracts, producing what is known as 
atrial systole, a period in which increased diastolic flow is again observed due to an increased atrio-ventricular pressure gradient. Finally, as the atrium relaxes, the ventricle experiences a second phase of roduced inflow, after which the cardiac cycle is finished and ready to be repeated. Figure 6 depicts the described phases of the cardiac cycle in diagramatic form.

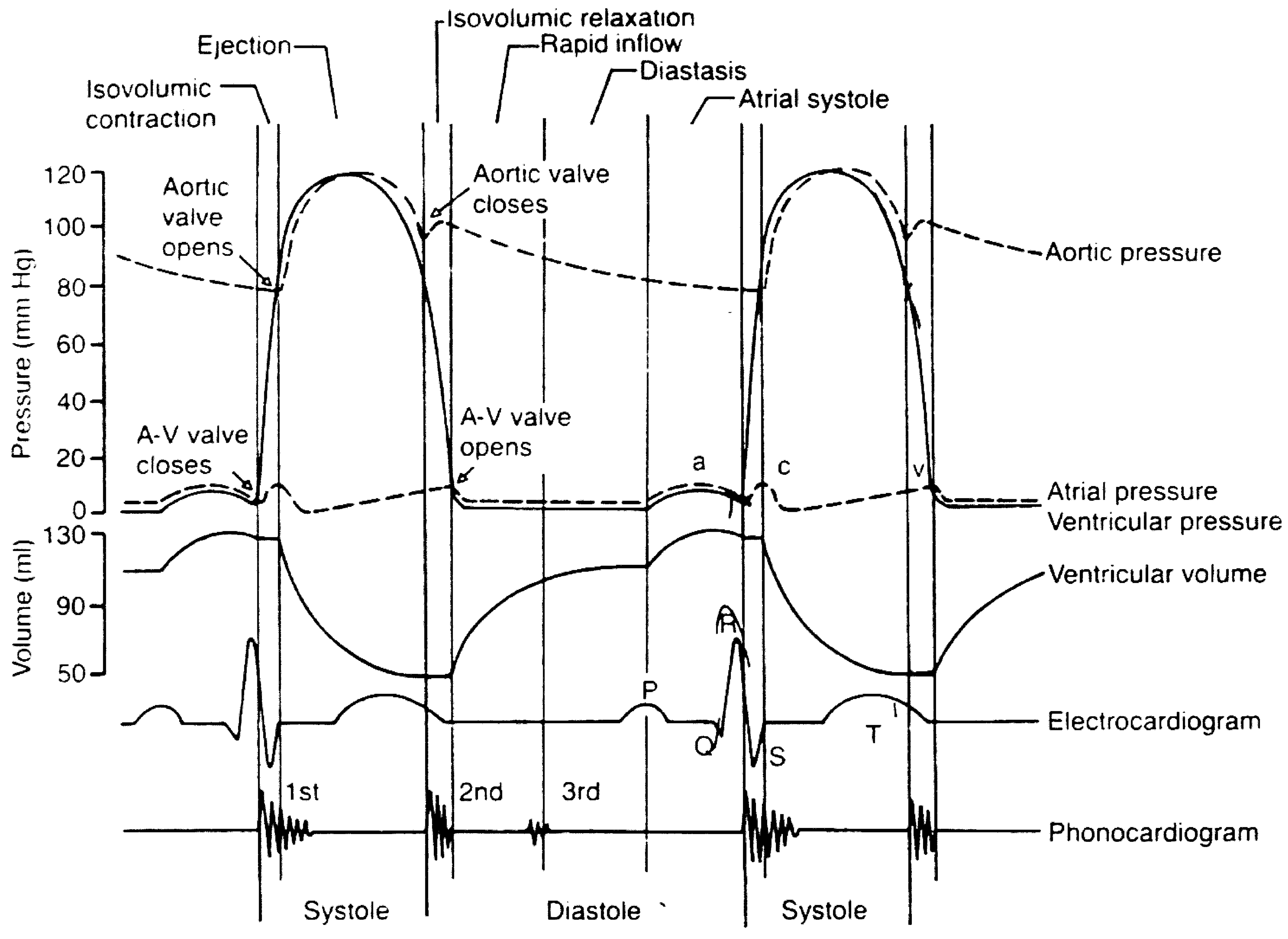

Figure 6: The events of the cardiac cycle, reproduced from Guyton [27]. 
What Weissler and Harris [20] call Total Electromechanical systole (TES) is what has been described as the first three phases of the cardiac cycle: isovolumetric contraction, rapid ejection and reduced ejection. TES can be easily obtained in vivo by simultaneous recording of phonocardiographic (heart sounds) and electrocardiographic activity (ECG). At the onset of ventricular depolarization, the ECG will show a voltage trace known as the eRs complex. Measuring the temporal interval between the QRS complex and the second heart sound, which represents the closure of the aortic valve, gives us the systolic interval. Observing 121 males and 90 females, who were supine and fasting, between 8:00 and 10:00 AM, and using linear regression to correlate the data obtained, Weissler et al. found the following equations between heart rate and systolic intervals:

$$
\begin{gathered}
Q S_{2}=-0.0021 \times H R+0.546 \quad \text { for males } \\
Q S_{2}=-0.0020 \times H R+0.549 \quad \text { for females } \\
\text { where: } Q S_{2} \text { is the TES (seconds) }
\end{gathered}
$$

By stipulating the cardiac frequencies of $50,72,100$ and 144 BPM, and using them with the average of the two above equations, we obtain systolic periods that, when divided by the cardiac period (I/HR) yields the percentual systolic interval. In summary, cardiac frequencies that are thought to 
be representative of the normal human range of operation were chosen and used to generate stroke volumes and systolic intervals. The cardiac output was obtained by the multiplication of heart rate and stroke volume. In order to simulate some degree of abnormality, once obtained, each cardiac output was increased and decreased by $25 \%$ of itself, thus producing twelve flow curves. The thirteenth flow curve was used to simulate what is thought to be representative of typical post-surgical conditions of cardiac operation. Shortly after open-heart surgery, as needed for valvular transplantation, the heart operates at low stroke volumes and consequent high frequencies. In order to investigate how each prosthetic heart valve would perform under such circumstances, a flow curve with a frequency of 150 BPM and cardiac output of only 3 Liters per minute was included in the experiments. With the above information, thirteen sets of parameters were obtained: 
Beart Rate Cardiac Output systolic Fraction stroke Volume

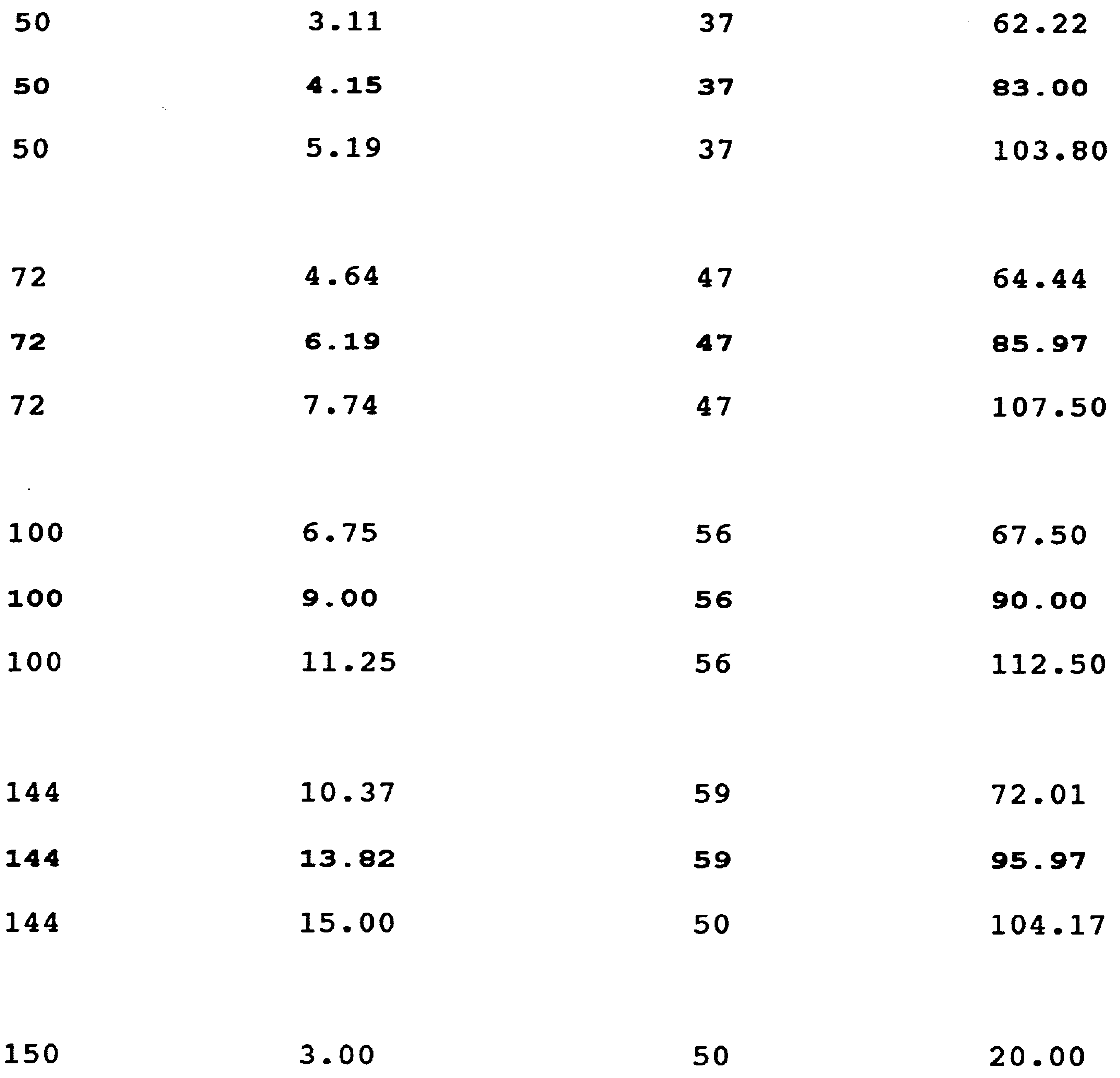

Table 1: List of parameters used to generate input flow functions. 
Physiological systolic and diastolic flow curves were obtained from Guyton [27] and normalized on the abscissa to the peak flow rate, for systole and diastole, and on the ordinate to the period. A total of twenty-three points were graphically extracted from the normalized curve (after photocopical expansion to minimize errors) and used in conjunction with a polynomial iteration routine, and the parameters above, to obtain the thirteen flow curves used in the experiments. The computer program used for the above purpose is capable of plotting ventricular volume, flow rate and acceleration. In addition, the shape of the flow curve can be easily changed in order to simulate pathological conditions, for instance, by simply changing the normalized locations of any of the twenty-three points used by the routine to establish the flow curve. A typical flow curve obtained in the manner described is shown in figure 7 . The first part of the curve represents systolic flow, while the latter part represents diastolic flow.

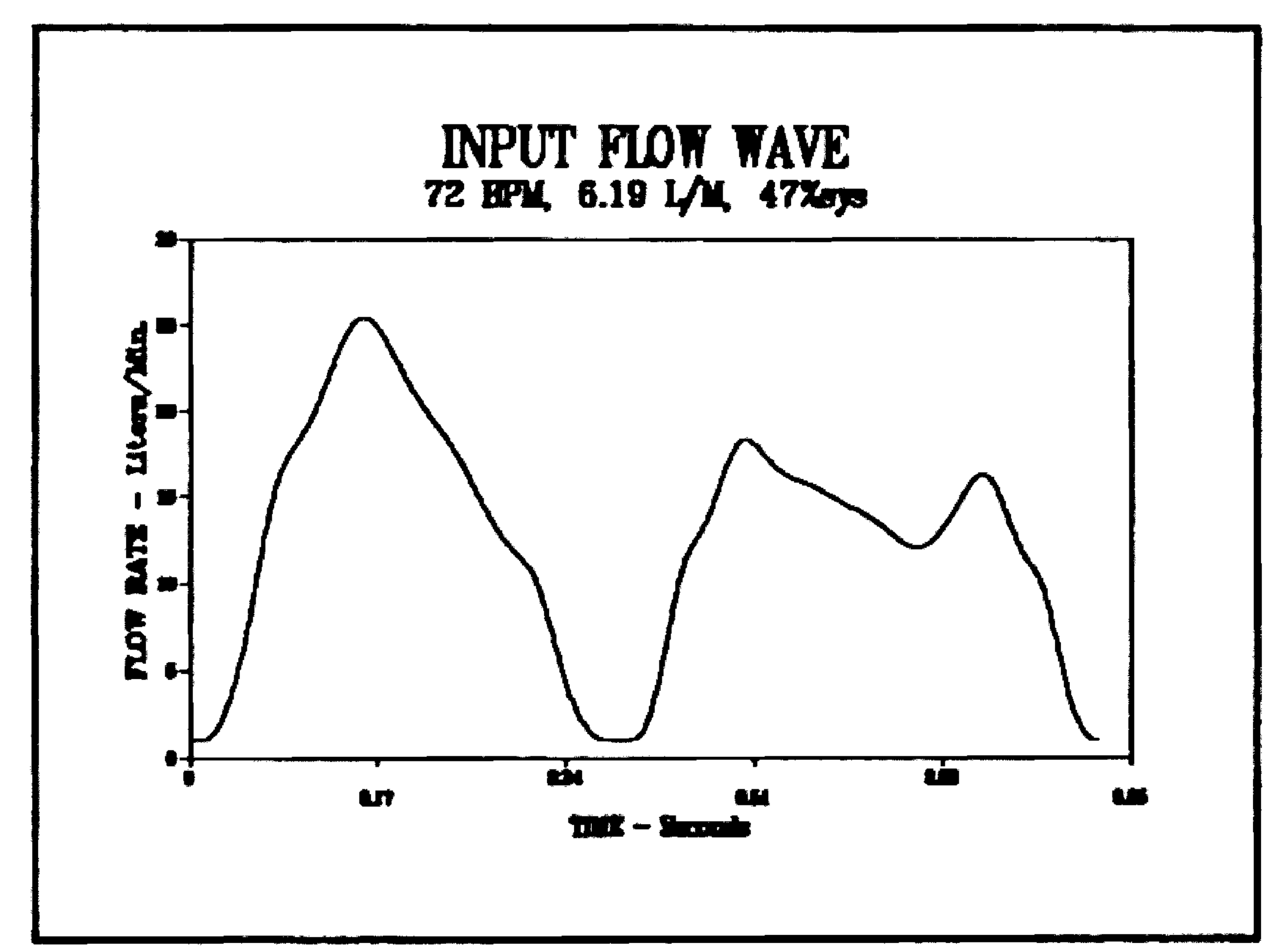

Figure 7: Typical input flow function. 
The introduction to this paper mentioned that this study would attempt to determine any possible variations in the coefficient of discharge of a particular heart valve due to its condition of operation. The relevance of studying normal, mildly or severely stenotic valves, as well as how each of these conditions were simulated in vitro, is now explained.

Patients that had prosthetic heart valves implanted become committed to a lifetime of periodic clinical examinations. In addition to wear of the mechanical or tissue device, other problems may arise that require explantation of the valve. A number of problematic conditions associated with the implantation of heart valves are described by Burch et al., and hence need not be duplicated here. [29] While some problems are of an exclusively biological nature, such as infectious processes and immunological rejections of the implant, others are purely mechanical, such as chipping of occluders that later caused embolitic processes (obstruction of small blood vessels, the brain is particularly succeptable to embolism due to the large number of micro vessels found there), or fracture at welds, with consequent instant death of the patient due to discontinued blood flow.

A third class of problem associated with prosthetic heart valves, and perhaps the most common, is one with a biological cause and mechanical consequence. Scar tissue has been noted 
to grow around the valve annulus and, if persisting, the tissue eventually prevents the valve from functioning optimally. [33] Severe transvalvular pressure drops have been observed in patients with scar tissue growth. Autopsies of such hearts reveal that the prosthetic valve became stenotic due to obstruction of movement of the occluder by scar tissue. since the growth of tissue around the valve would result in increased transvalvular pressure drop, it could be diagnosed by the orifice area method, provided that knowledge of the correct value for the coefficient of discharge exists. Smaller than actual values for $\mathrm{Cd}$, for instance, would produce EOAs that are larger than actual and thus do not reflect possible valvular stenoticity.

In order to investigate the possible variation of the coefficient of discharge as a function of stenoticity of the valve, all six (three types, two sizes each) valves were tested under normal conditions, as well as each of two simulated degrees of stenoticity: mild and severe. Implementation of the stenotic condition was accomplished by bracing the occluders of each mechanical heart valve (MHV) with a stainless steel wire $(d=0.025 ", 0.635 \mathrm{~mm})$ in such a manner that the occluder's motion was obstructed. The tissue valve (porcine bio prosthesis) was sutured along the commissures in a manner similar to what would happen if calcification of the commissures were present. Figure eight depicts the simulated conditions of stenosis. 
Condition of Operation: Normal, Mildly and Severely Stenotic Bileaflet (st. Jude Medical)

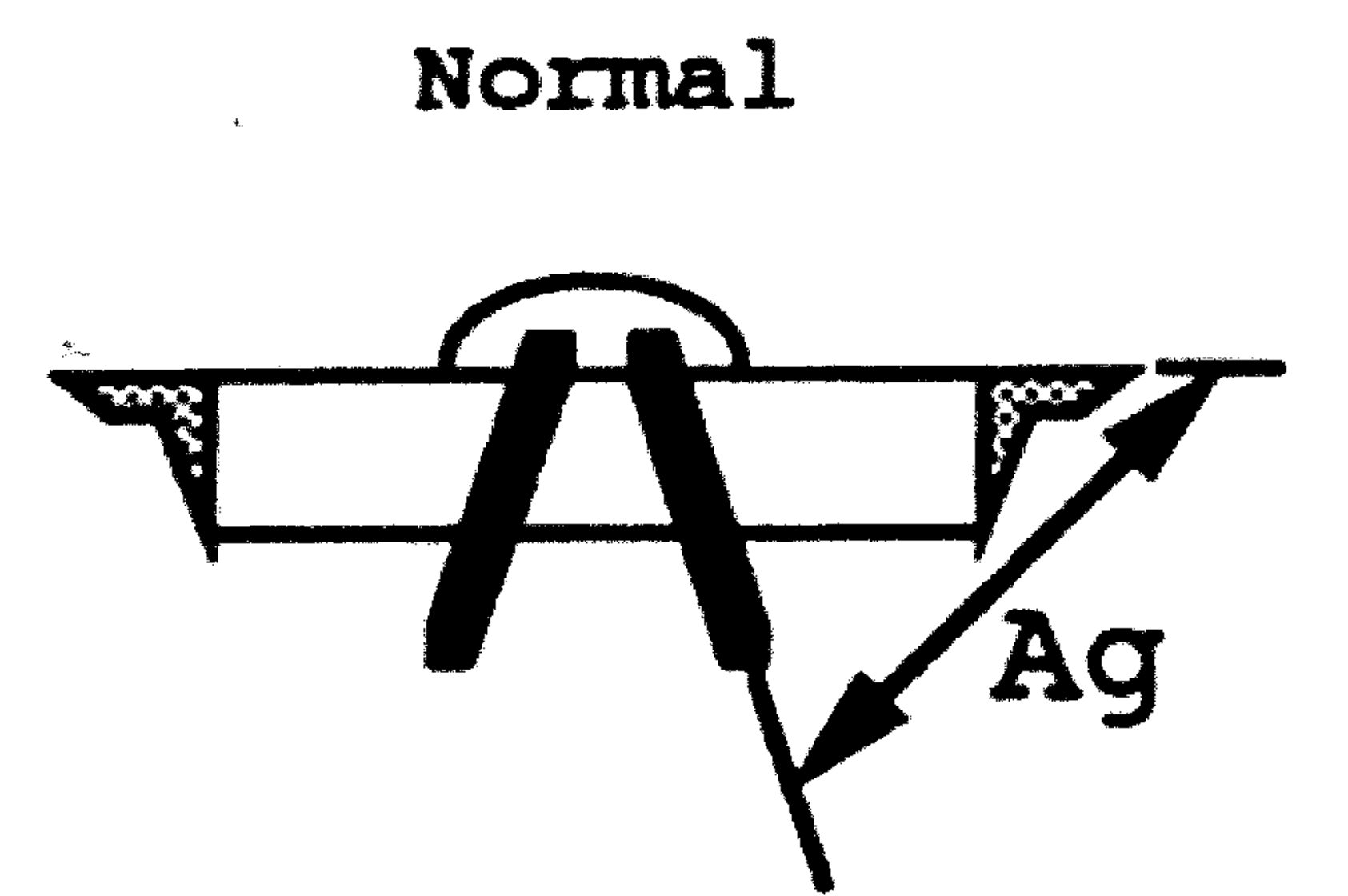

$27 \mathrm{~mm}$

$\mathrm{Ag}=85.3$ $\mathrm{Oa}=3.25$

$29 \mathrm{~mm}$

$$
\begin{aligned}
& \mathrm{Ag}=84.8 \\
& \mathrm{Oa}=3.71
\end{aligned}
$$

\section{Tilting Disc} (Medtronic)

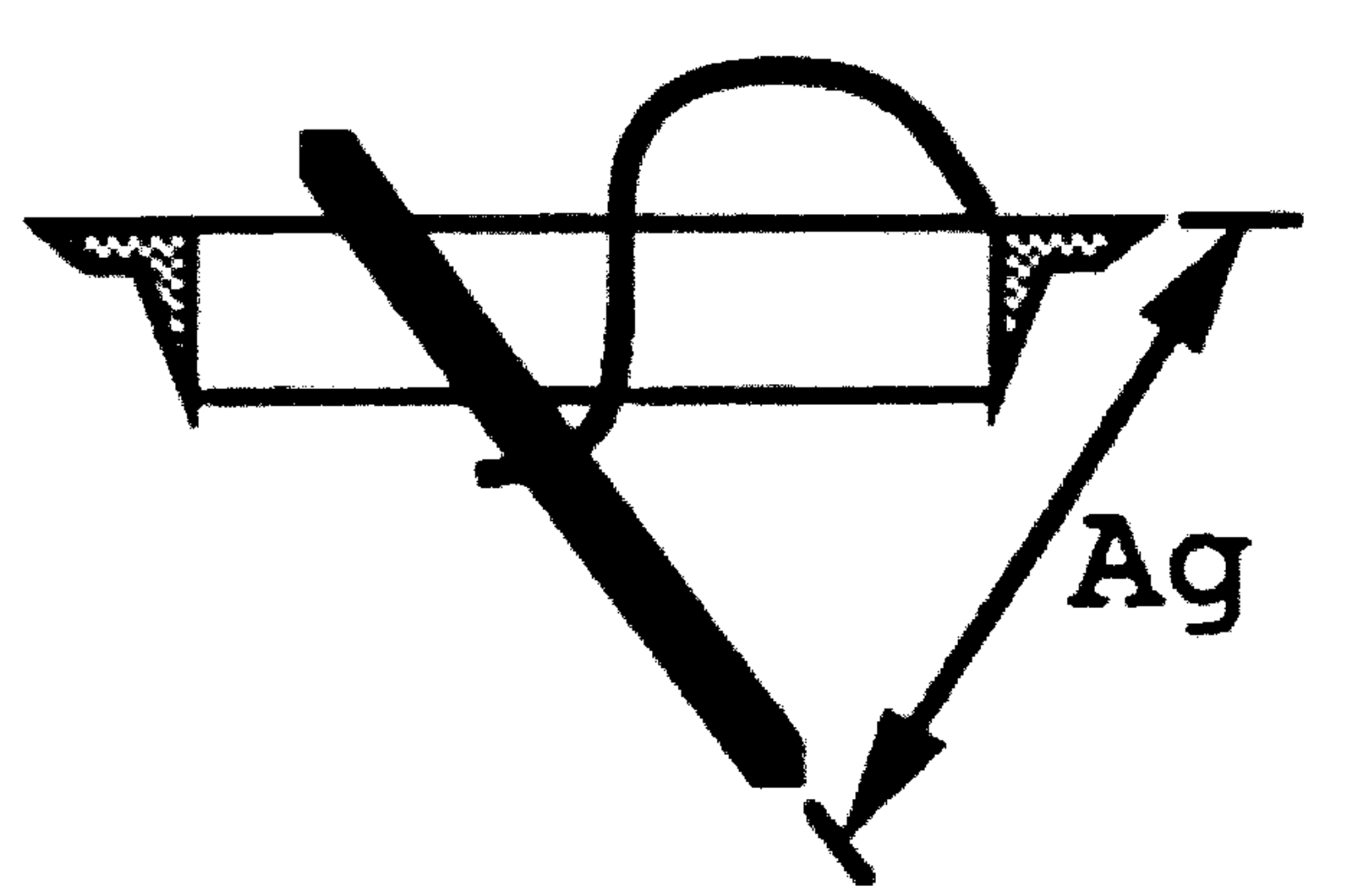

$$
\begin{array}{r}
27 \mathrm{~mm} \quad \mathrm{Ag}=61.6 \\
\mathrm{Oa}=1.79
\end{array}
$$

$29 \mathrm{~mm}$

$$
\begin{aligned}
& \mathrm{Ag}=65.1 \\
& \mathrm{Oa}=2.40
\end{aligned}
$$

Mildly Stenotic

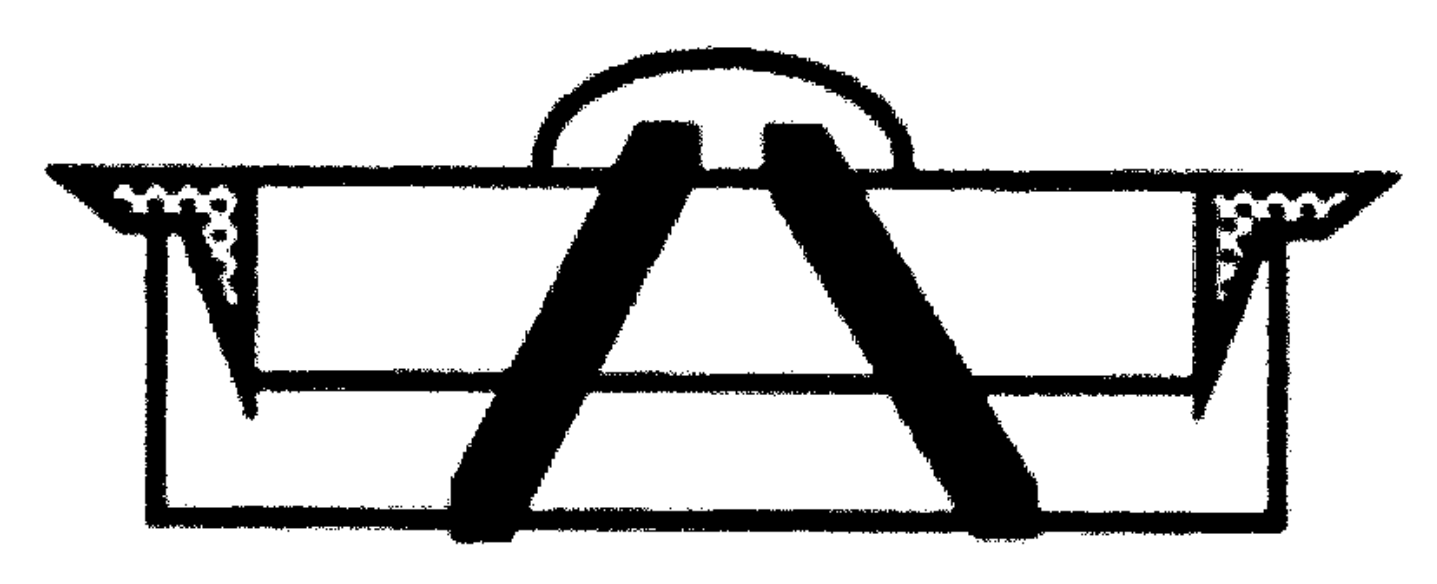

$\mathrm{Ag}=63.9$

$\mathrm{Oa}=1.92$

$\% N=59$

$\mathrm{Ag}=56.7$

$\mathrm{O} a=1.77$

$\% N=48$

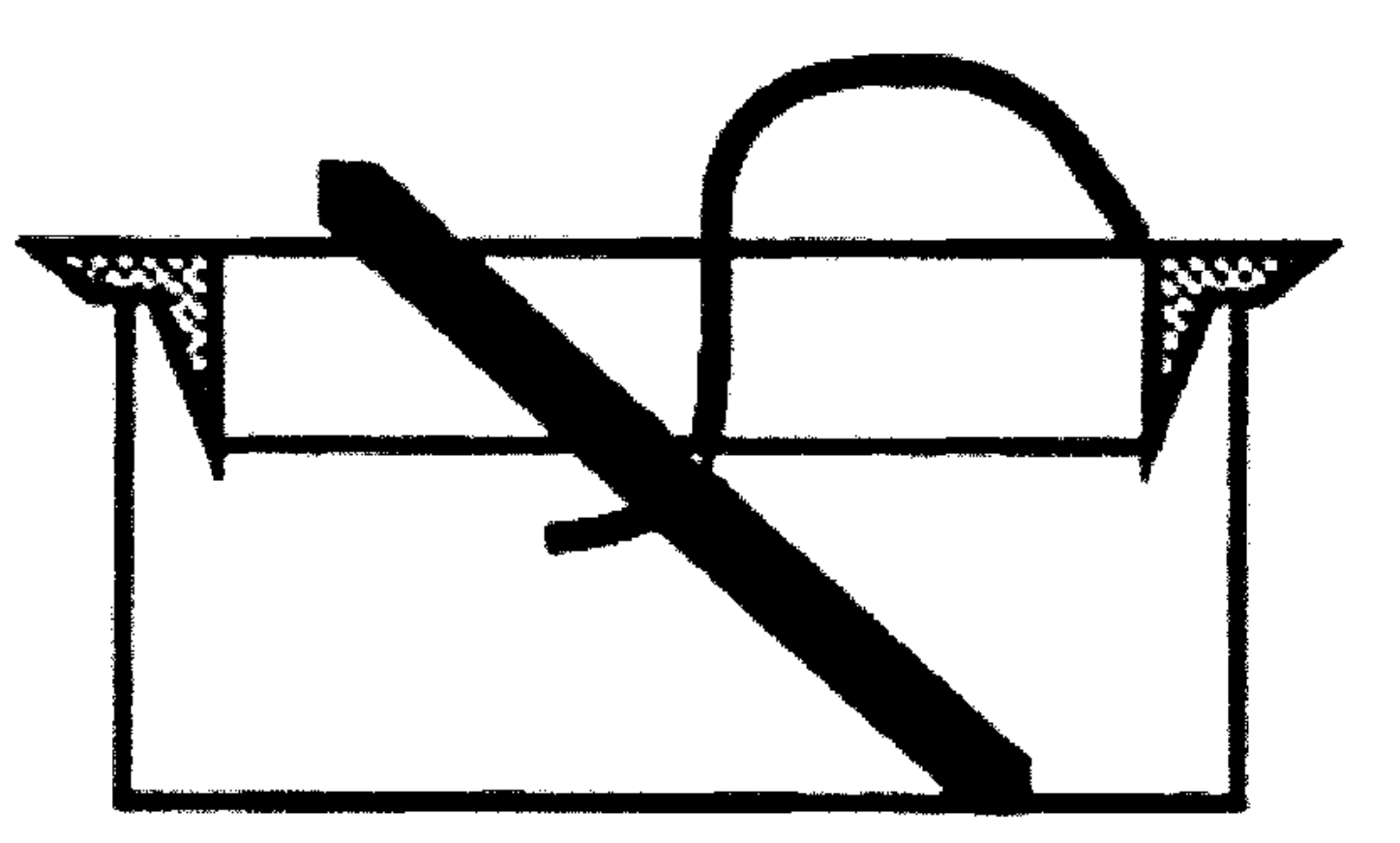

$$
\begin{gathered}
\mathrm{Ag}=49.5 \\
\mathrm{Oa}=1.18 \\
\% \mathrm{~N}=66
\end{gathered}
$$

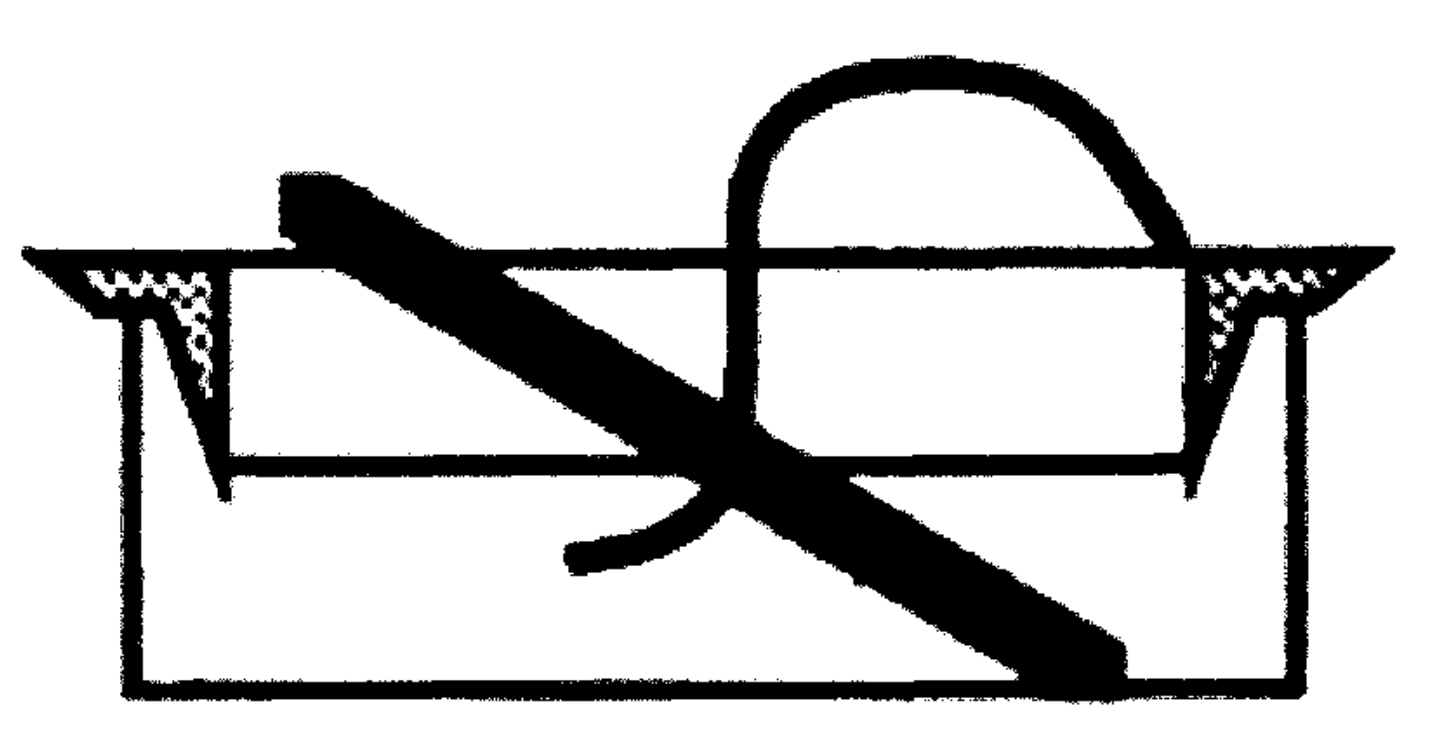

$$
\begin{aligned}
& \mathrm{Ag}=45.1 \\
& \mathrm{Oa}=1.28 \\
& \% N=53
\end{aligned}
$$

Severely Stenotic

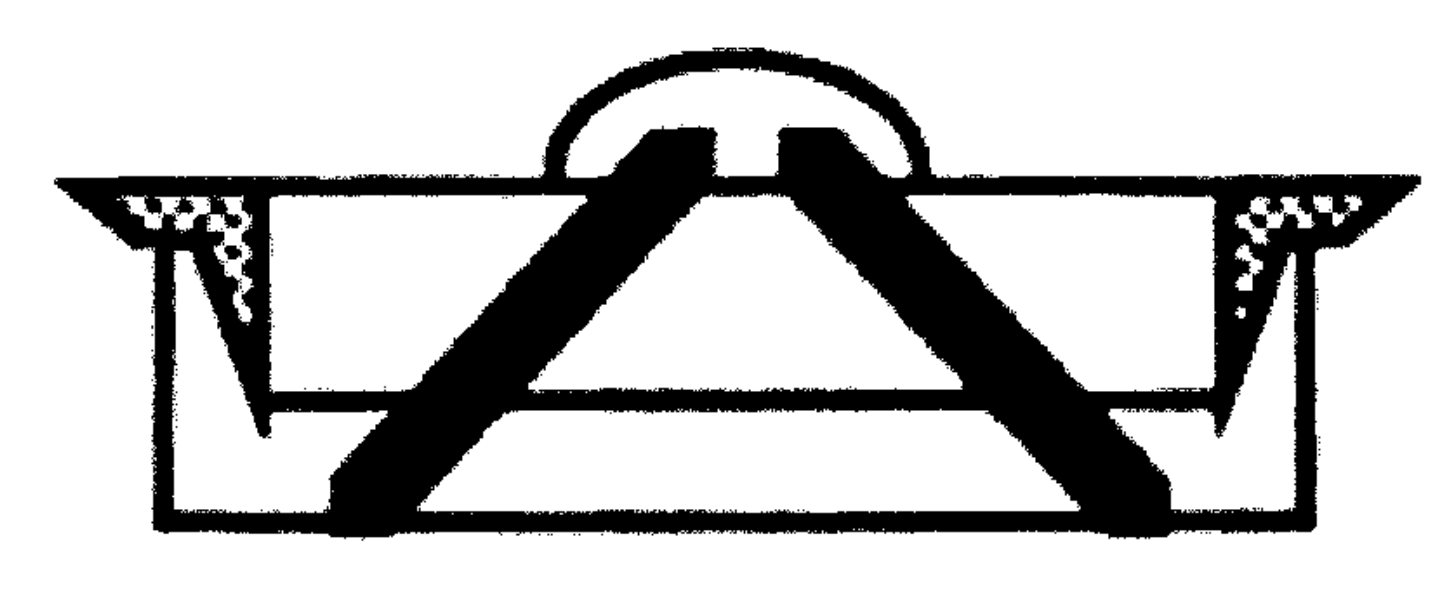

$\mathrm{Ag}=45.8$

$\mathrm{Oa}=0.93$

$\% N=29$

$\mathrm{Ag}=43.0$

$\mathrm{Oa}=0.87$

$\% \mathrm{~N}=24$

Areas are in centimeter square - Angles in degrees from Borizontal. Bio Prosthetic (Carpentier-Edwards, 27 and $29 \mathrm{~mm}$ ) valves were sutured along commissures to produce stenoticity.

Figure 8: Quantitative information and depiction of valve condition. 


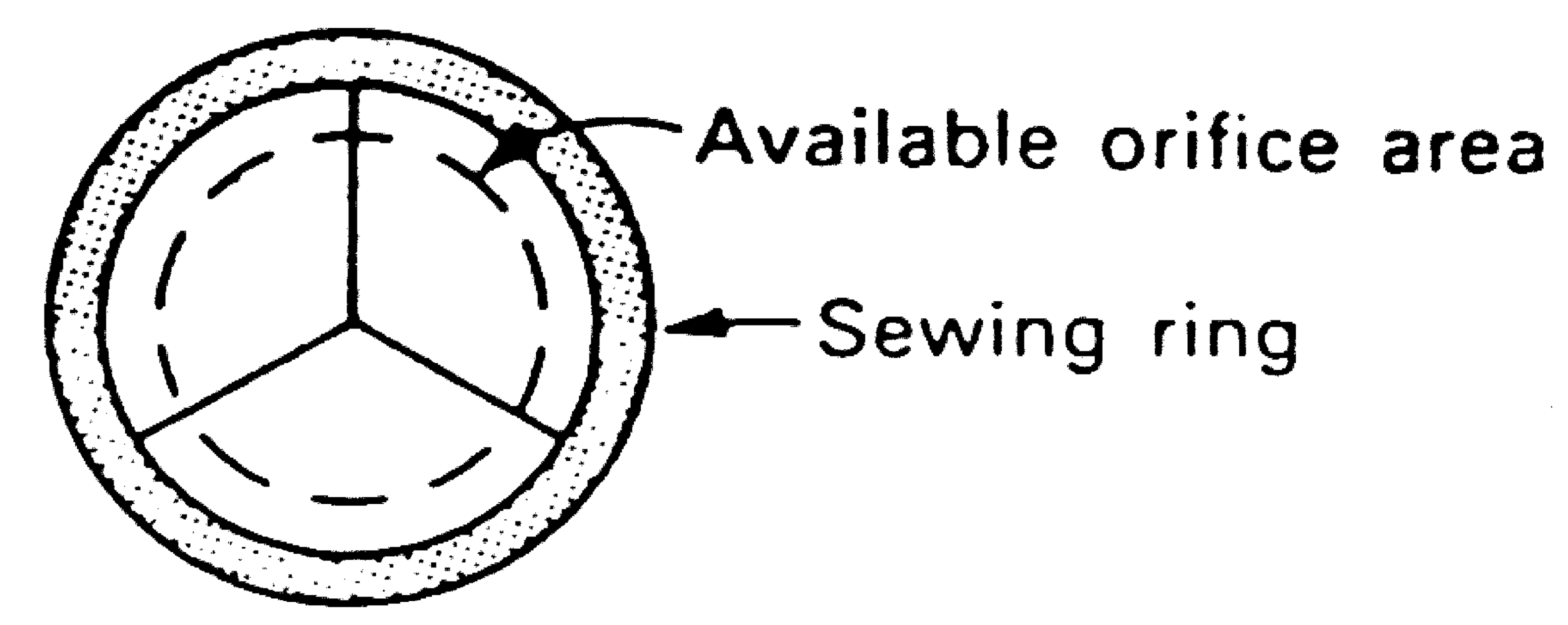

Normal (Nonstenotic) Valve

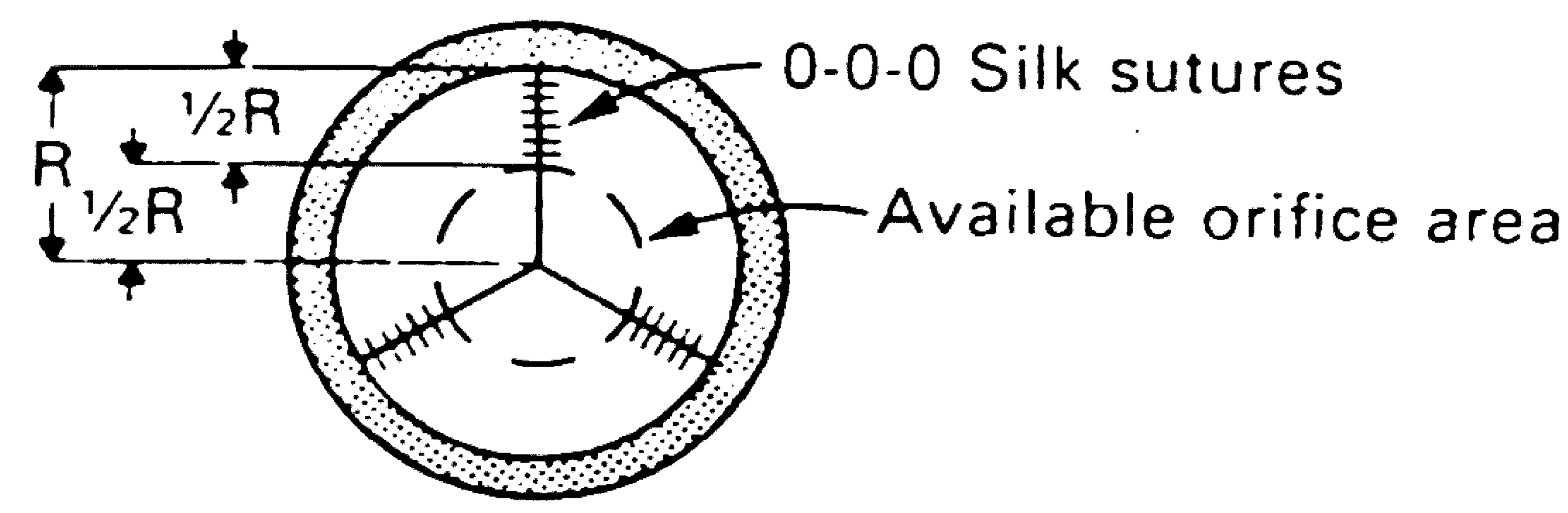

Mildly Stenotic Valve

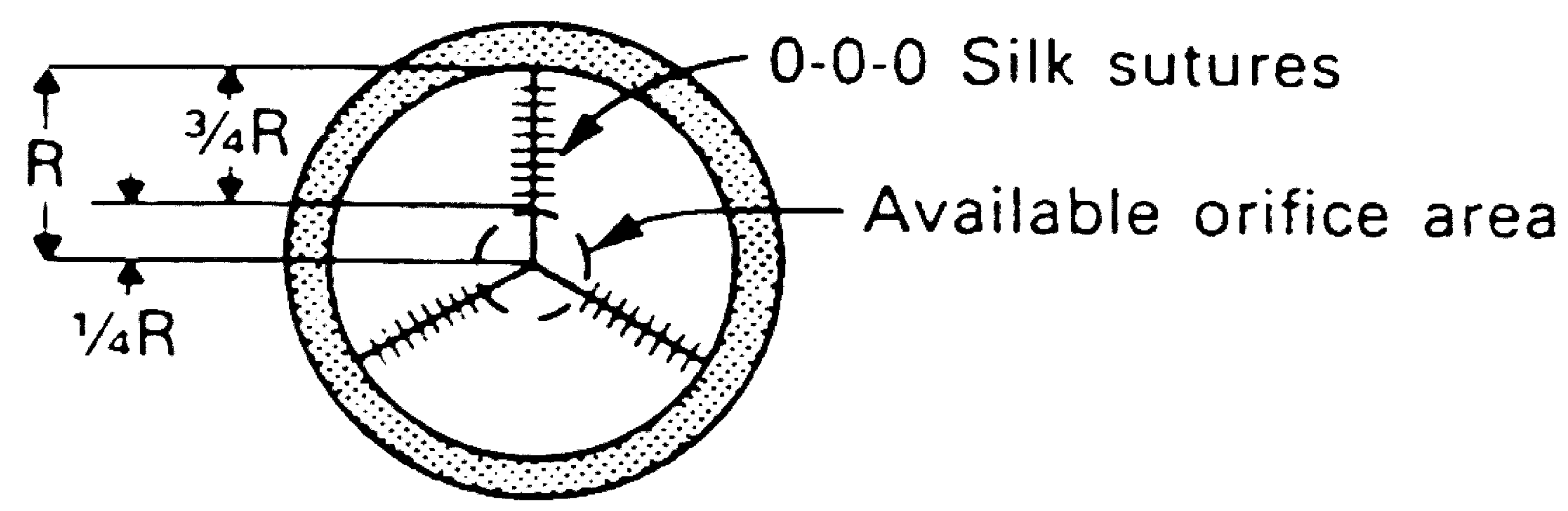

Severely Stenotic Valve

Figure 9: Representation of the method employed to simulate stenoticity in tissue valve. Adapted from Schoephoerster [14]. 
The first part of this project was the design and construction of the test equipment itself. There are five main groups of components to the CVD system: The pulse duplicator, the cardiac emulator, the systemic simulator, the ventricular system and the data acquisition system, all of which were described before. Detailed technical description and design information on the CVD is provided elsewhere, thus it is judged superfluous here.

Once the construction of the machine was finished, a period of six months of intense validation and calibration followed. Pressure wave-forms were not considered close enough to physiological, hence continued modifications of the system, yielding several dozen configurations which were tested. Once the machine was considered to "duplicate" the human cardio vascular system, experiments began. Figures ten and eleven depict the ensemble average of twenty strokes of the CVD, and the raw data for three consecutive strokes, respectively. The reason for showing such pictures here, which will be shown again and explained with the results, is to validate the claim that the machine does, in fact, produce physiological pressure wave-forms. Figure ten shows how repeatable, from stroke to stroke, the machine's output is. Figure twelve was copied from a physiology textbook (Guyton [27]); it is included here for comparison with the curves produced by the FIU CVD. 


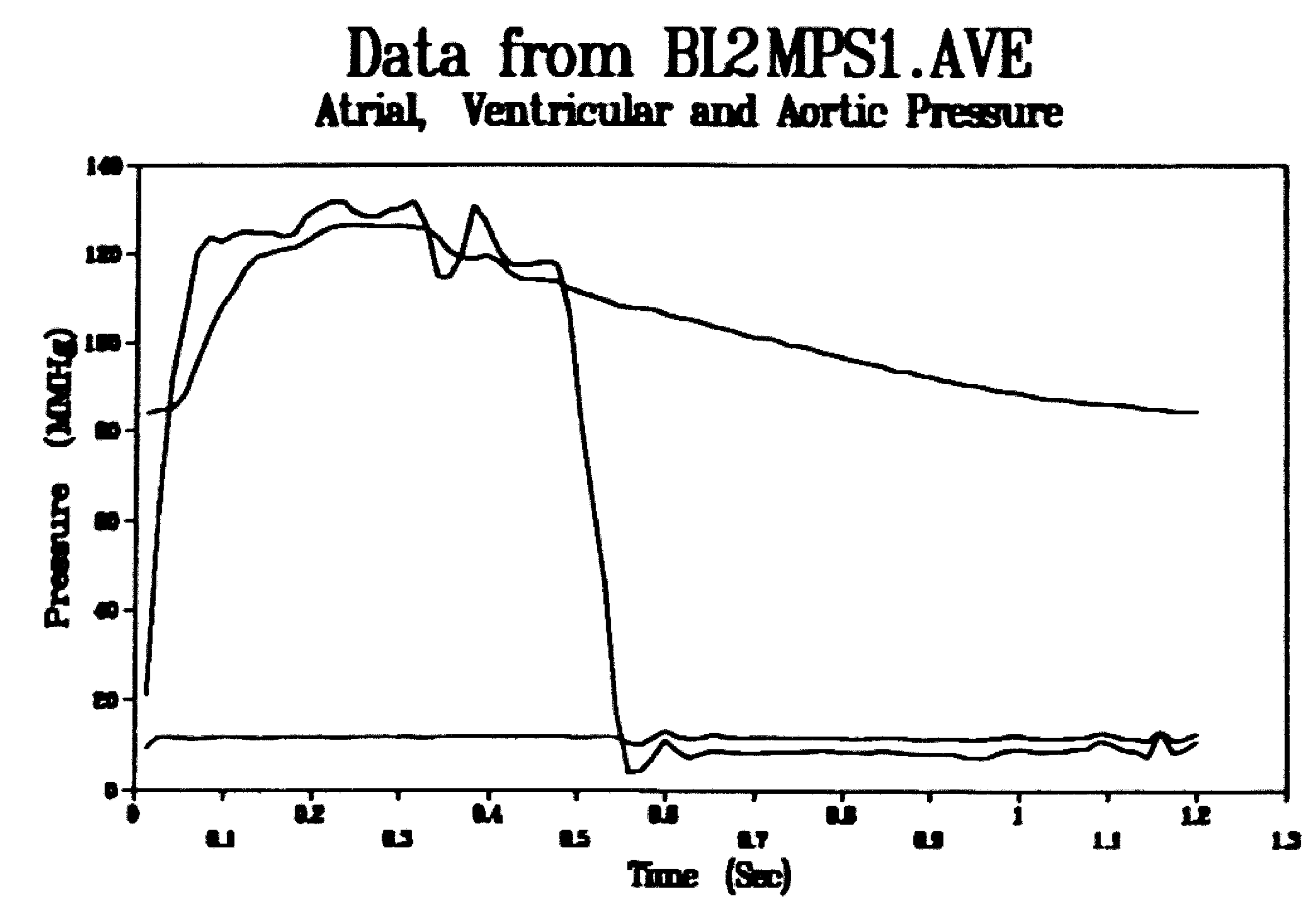

Figure 10: Atrial, Ventricular and Aortic pressures; figure represents the average of 20 strokes.

Data from BL2SPS1.DAT

Atrial, Ventricular and Aortic Pressure

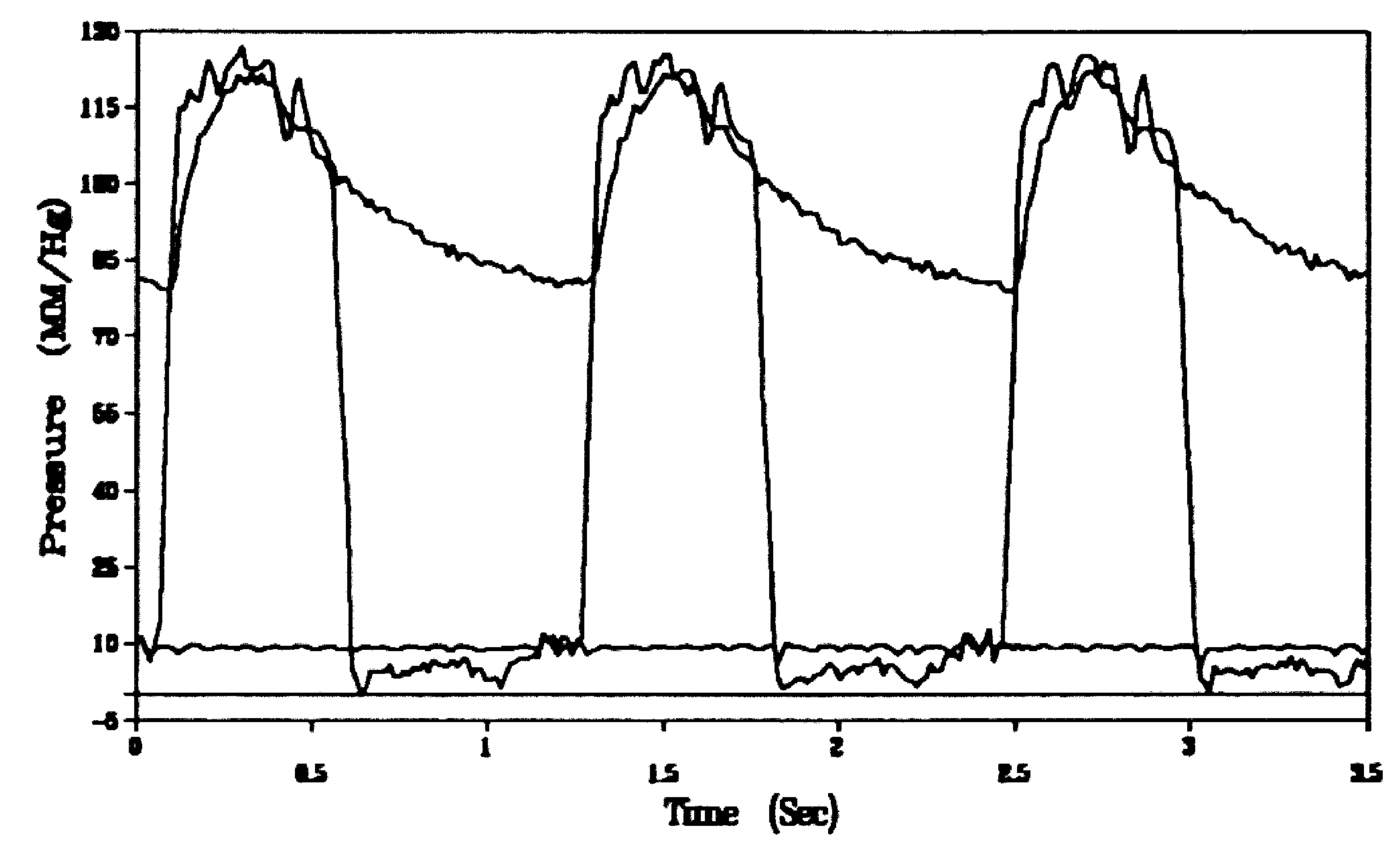

Figure 11: Atrial, ventricular and Aortic pressures for three consecutive strokes. 


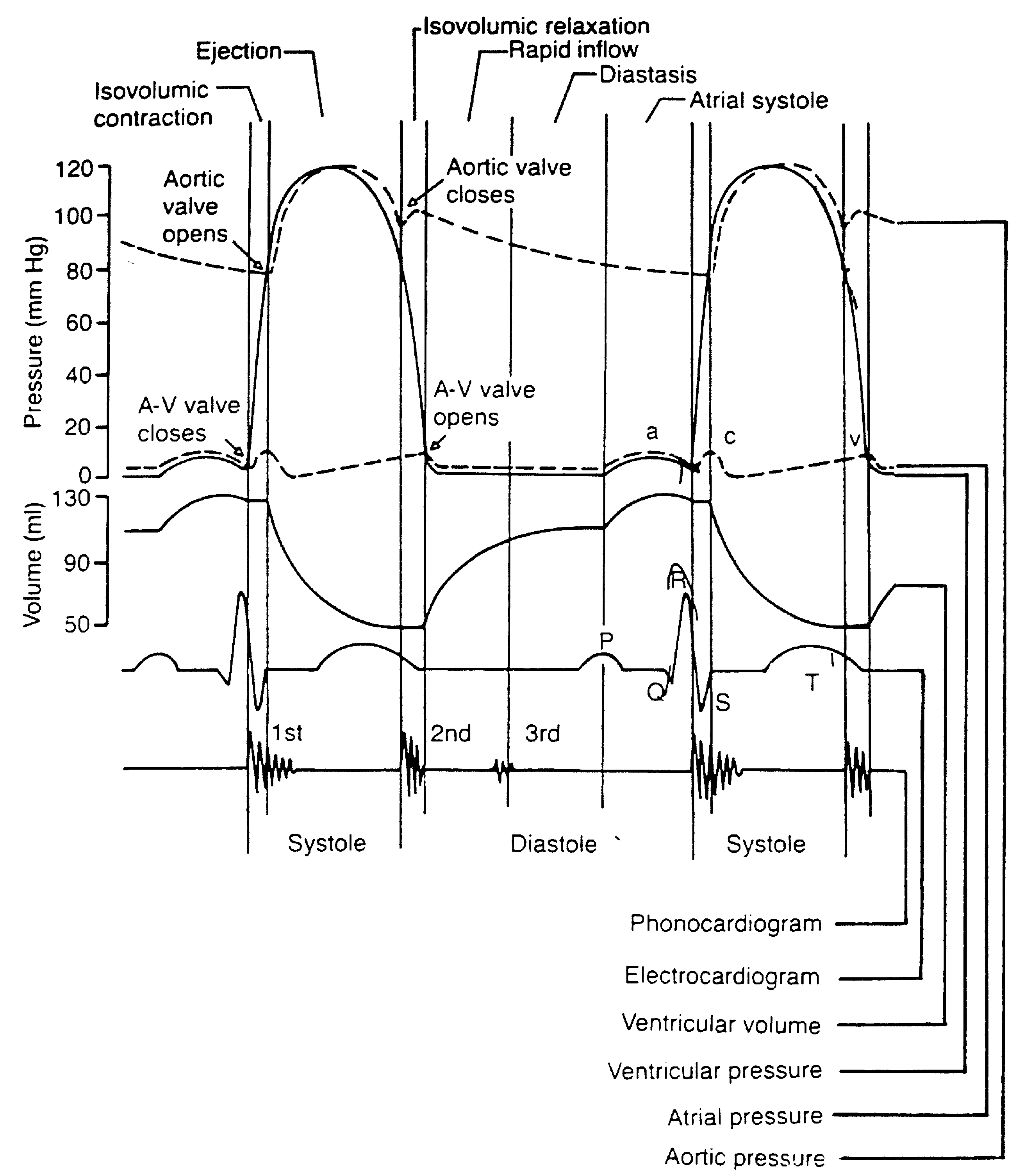

Figure 12: Pressure curves obtained from the Guyton Textbook of Medical Physiology [27]. The reader is invited to note how well the pressures obtained from the FIU CVD match physiological ones. 
Validating the machine's ability to duplicate physiological flow and pressure wave forms by virtue of observing the pressure wave-forms produced by the very machine in question demands validation of the pressure transducing apparatus and arrangement. The pressure measuring equipment used for this experiment is FDA approved for human use and has been thoroughly tested and validated for the past fifteen years. [17] [26] One important reason for using medical equipment, even though it costs many orders of magnitude more than conventional equipment, to measure flow and pressures is that whatever the errors inherent in the system are, they would also be seen in a clinical situation where the same equipment is used. Consequently, values for the coefficient of discharge determined based on measurements made at our laboratory, even if slightly in error would be valid at other laboratories that use the same measurement equipment. Here, only errors that are inherent in the design of the measuring equipment are being referred to, which are minimal. Errors associated with the use of the equipment, such as those caused by erroneous calibration, have been kept to a minimum, since strict calibration procedures were carried out.

The measurements of pressures within a fluid in motion must address the possibility that dynamic, in addition to static, pressures are being measured. The physiological pressures that were emulated are static pressures, which means that the pressure transducing elements or the machine must be 
kept away from the flow stream, where impact with fluid particles would cause higher than actual pressures to be read, if the element is normal to the flow, or lower than actual pressures, if the element is parallel to the flow. Reeping the transducer far enough away from the flow stream brings in the question of the possibility of large pressure gradients within the flow field, which would, again impair the measurement of pressures.

The final locations, for many were tried, of each of the three pressure transducers utilized were chosen with the above considerations in mind. The atrial pressure, the pressure upstream of the mitral valve, and the ventricular pressure, just downstream of the valve, are the important ones for this study because they are used to compute the transvalvular pressure drop. The use of the aortic pressure was incorporated, not because it is needed for the determination of the coefficient of discharge, but instead because it gives us the quickest indication that the machine is operating under conditions of physiological similitude, since it is the aortic pressure, measured at the brachial artery, that is used to indicate cardiovascular function. Figure thirteen depicts dimensional details of the locations of the pressure transducers. 


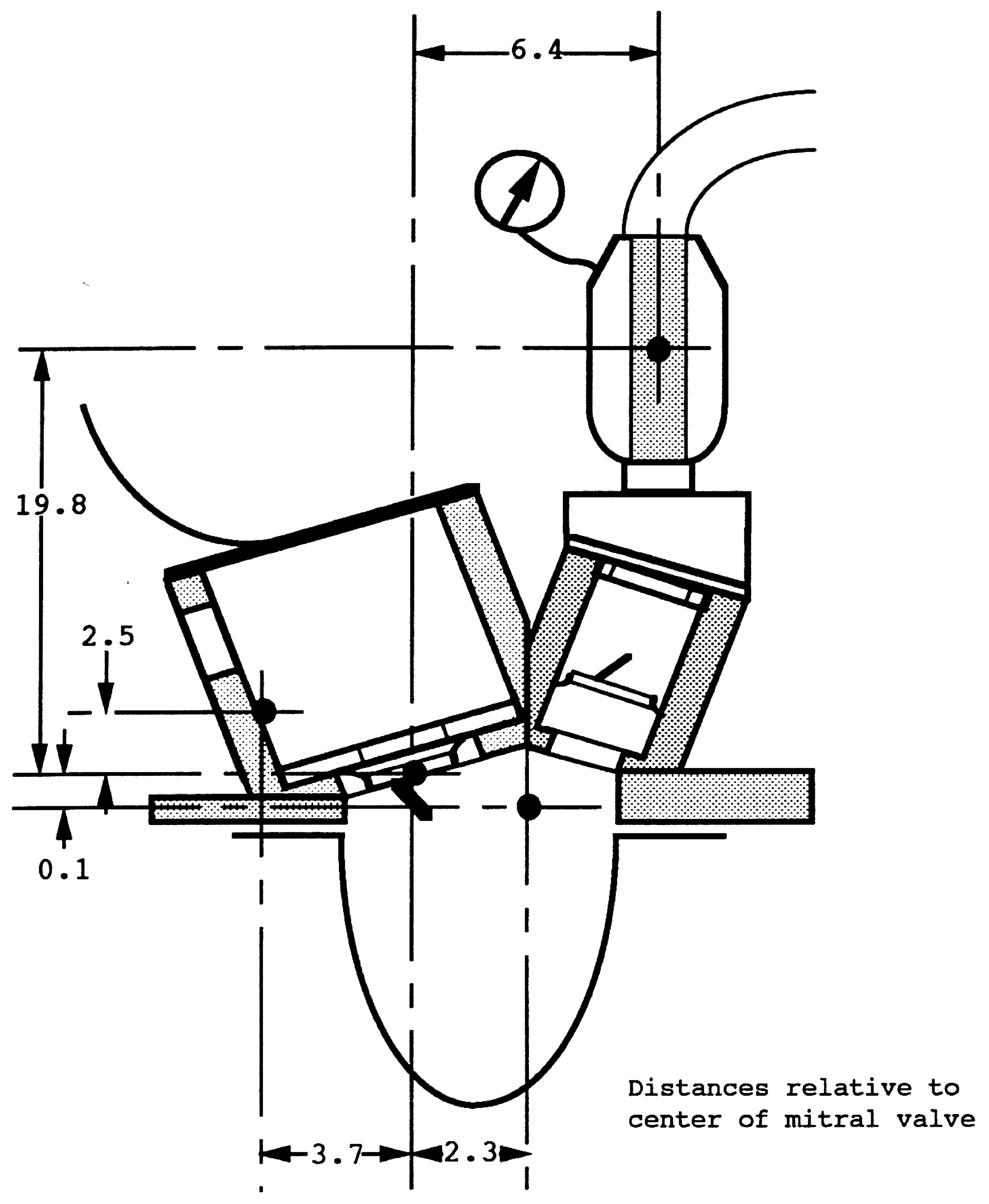

Figure 13: Locations of Atrial, Ventricular and Aortic pressure sensors relative to mitral valve. 
Once the correct flow wave forms were generated using the polynomial iteration routine described above, they were validated by placing the probe of the meter at the output of the Pulse Duplicator and recording the flow wave forms there. Figure fourteen shows the setup used to validate the output of the pulse duplicator.

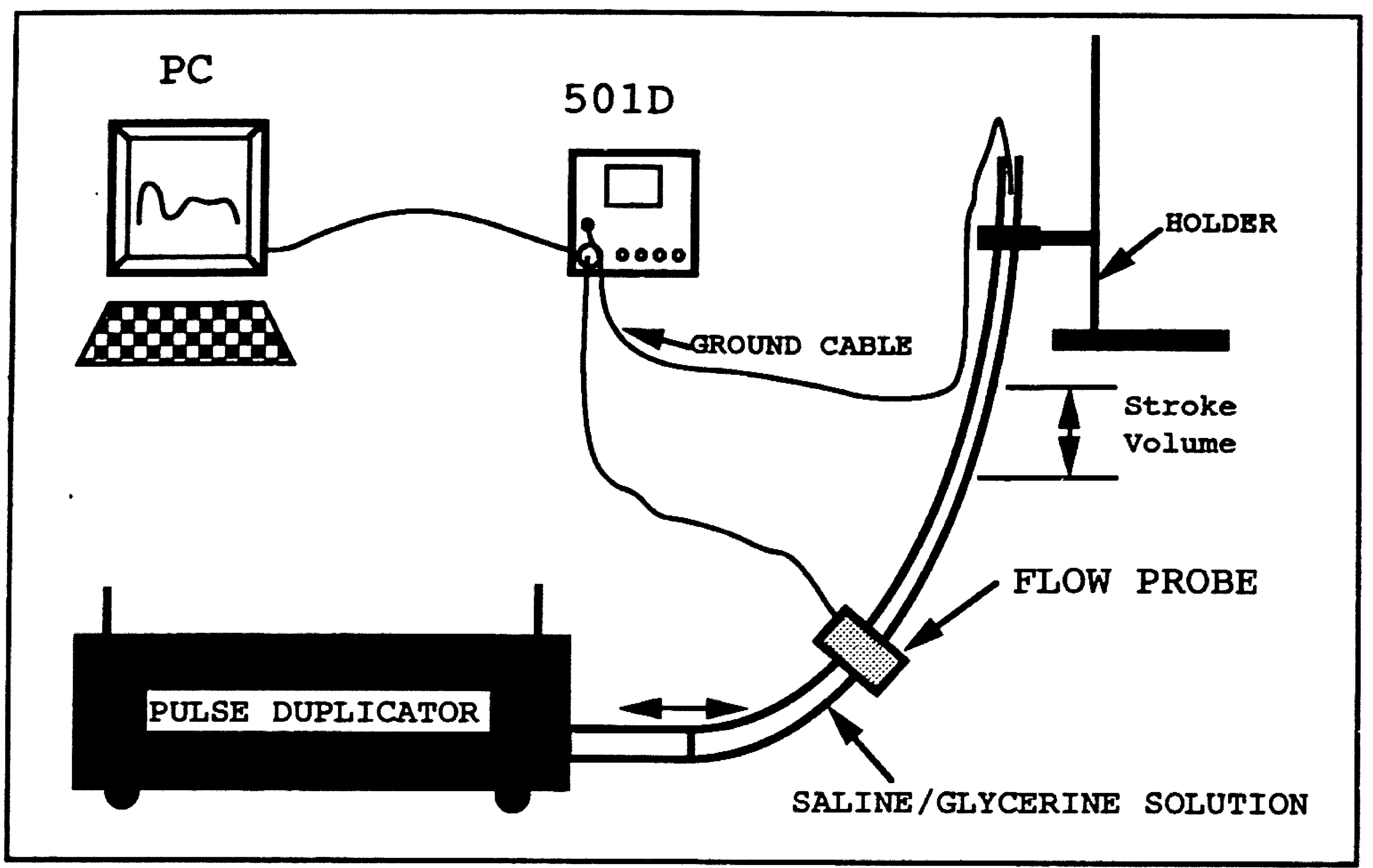

Figure 14: Experimental setup used to validate $P D$ output flow rates.

Virtually no error was found between the numerical input to the motor and the dynamic output of the PD, as expected, for the following reason: A stepping motor advances one angular step of 1.8 degrees every time it is reached by a suitable voltage spike, in this case 60 Volts in amplitude and a minimum of 1 microsecond in duration. The motor is made to 
run continuously by providing it with a series of pulses; its velocity is determined by the temporal interval between pulses, and its angular displacement is controlled by the number of pulses sent. The motor and controller used in this machine also allow for what is called microstepping, or the ability to run the motor at steps smaller than 1.8 degrees, or two hundred steps per revolution. 1000,5000 and 25000 steps per revolution are also possible, allowing for enhanced positional and velocity control. Since the motor can only advance one angular step (motor was run at 1000 and 5000 steps per revolution) per electrical pulse received, and since the temporal length of each step is known, the determination of the motor's velocity becomes a simple matter of determining the temporal delay between two consecutive pulses. The resolution, ability to control the delay, is within 0.5 microseconds. The frequency of pumping was also measured to ensure precision. The signal from the 'home' switch was examined on an oscilloscope, the frequency of the square wave produced is equal to the pumping frequency. Again, absolute correction was found. In addition, both flow and pressure control units, in strict adherence to the manufacturers' procedures, were calibrated each time a measurement was taken.

It is not the flow rate at the outlet of the PD that is needed to compute the $C d$, however; instead, mitral flow is needed. Although all effort was exercised to measure mitral flow directly with the small flow probe available (50 $\mathrm{mm}$ 
circ., $d=5 / 8$ inch), the additional resistance the probe imparted on the flow was sufficient to distort the pressure wave-forms. Needless to say, this problem is a special characteristic of pulsatile flows, where any change in fluid quantity (inertance), compliance or resistance will completely change the behavior of the system. This fact may not be so obvious to the reader familiar only with steady state flows, in which case the author suggests that the reader reviews the principles of capacitive-resistive-inductive electrical circuits exposed to alternating currents. In view of the above difficulty, a fact was brought to light that allowed for accurate knowledge of flow rates without the need for direct measurement: since we have a positive displacement system, and if the aortic valve operates correctly, as it did, then the flow rate imparted onto the fluid by the retracting piston (PD) is, by necessity, the mitral flow. To suspect the opposite would be to deny the principle of continuity as applied to an incompressible fluid.

Careful inspection of the schematic diagram of the CE and of the PD (figures 4 and 5) will reveal that when the piston is retracting (going towards the 'home' position, previously defined to be at end-diastole) the ventricular sac is expanding, which requires that fluid enters the ventricular cavity. Since there are only two openings at the base of the ventricle, one for the mitral, the other for the aortic valve, and since the aortic valve snaps shut as soon as the piston 
begins its retrograde motion, all the fluid entering the ventricle during this period must pass through the mitral valve. Consequently, it is postulated that for a positive displacement machine of the type employed for these experiments, transvalvular flow, either mitral or aortic, does not need to be measured when normal valves are employed, rather, the input flow rate can be correctly used as the valvular flow.

The reader must keep in mind two important points regarding the use of input flow rates in the formulations for the coefficient of discharge. The first is that this flow is completely independent of the passive characteristics of the systemic simulator, within which pressures developed only are a function of the complex load. In the cardiac emulator, however, a simple case of continuity is observed. The second point to keep in mind is the relative low reliability of the flow rate measurements that are possible clinically, the application of this project. Since most clinical laboratories use either the Fick method of oxygen consumption or the diedilution method to estimate flow rates through the heart, it is clear that the resultant calculation of effective orifice area can only be as accurate as the flow estimate used. This work, therefore, using controlled experiments and positive displacement equipment is, by necessity, many orders of magnitude more accurate than the application for which it is intended. 
Being satisfied that the machine is able to simulate physiological pressure and flow waves, and that the measurement of these parameters is precise and accurate, experiments were begun. The blood analog fluid was 368 aqueous Glycerine solution. This mixture produces a calculated density of 1.092 grams per cubic centimeter and a viscosity of $3.5 \mathrm{cP}$, both similar for the values for whole blood. (centipoise) [14] [15]

Each of the two hundred and thirty four experiments consisted of the same procedure. The mitral valve was sewn into the valve holder of the CVD and loaded into the atrial cavity such that the axis of tilt of the leaflets was perpendicular to the anterior-posterior (AP) axis of the CVD for the bileaflet valve, and parallel to the same for the tilting disc valve. Tissue valves were mounted without regard to position since their opening is approximately circular.

Once the PD was put in motion, a period of three to seven minutes of stabilization and adjustments followed. Small changes in the resistors and compliance chambers helped to produce pressure tracings that are close to ideal. Up to two channels, typically aortic and ventricular pressures, were displayed by the storage oscilloscope at a time, (Hameg HM 205-3) until the desired outcome was reached, then recording of the data begun. Atrial, ventricular and aortic pressures, and piston position, were recorded simultaneously by the DA board for at least twenty strokes, at eighty to ninety points 
per stroke. The data file was analyzed by a computer program to average the pressures according to the position signal present in the data stream. Ensemble averaging produces pressure data for a single stroke, where each data point is the average of twenty pressures at the same time in different strokes. The average pressures were plotted for final certification that the data acquired met quality standards and the trans-mitral pressure drop was computed; the mean, and that at the time of peak flow rate.

Mechanical heart valves open fully early in the diastolic period and remain open throughout it. In fact, complete and consistent opening of the valves is one indication that the CVD is operating under conditions of physiological similitude. Only one picture of each mechanical valve, under each condition, is necessary for all thirteen flow rates used with that valve. The tissue valves, on the other hand, cannot be relied upon for open area consistency; consequently, each of the 78 experiments with tissue valves were photographed separately with a Panasonic PV 760 camcorder for about ten cardiac cycles. The tape produced was then played on a 29 inch monitor and the motion of the valve observed, frame by frame. Once a frame, thought to be representative of the opening of each valve, was chosen, a frame grabber connected to the VCR (Panasonic AG 6500) was used to store that image on a computer disc. Each of the 90 pictures was then printed, 78 for the tissue and 12 for the mechanical valves, upscaled by a nominal 
factor of six. A planimeter was then used to measure the opening area. (Reuffel \& Esser 620000) Each area was measured a minimum of three times, some more, until consistency was achieved, and the resultant average determined. This average area was then divided by the true scale factor for each picture in order to yield true valve area. The true scale factor is found by measuring the dimension of the valve's diameter in the picture and dividing it by the measured diameter of the valve. This method always produces the projected open area of the valve, which may be the source of some errors as will be discussed later.

Each of the 234 pressure drops ( 3 valves, 2 sizes, 3 conditions, 13 flow rates), their corresponding mean, RMS and peak trans-mitral flow rates and the valvular areas were inserted into a spreadsheet (Quattro) to calculate and display the four correction parameters described earlier: the Gorlin coefficient of discharge, the Aaslid effective area index, the Gabay coefficient of discharge and the Gabay performance index. The spreadsheet also produced a file that was read by another computer program that was used to statistically analyze and display the parameters. 
The three types of mitral valve used for these experiments are: the St. Jude Medical, Bileaflet valve; the Medtronic Hall, Tilting disc valve (also known as the monoleaflet design); the Carpentier-Edwards porcine tissue Bio prosthesis. All three valves were used in the sizes of 27 and $29 \mathrm{~mm}$. Figure fifteen shows the bileaflet and tilting disc designs; a high-quality, reproduceable picture of the tissue valve is not available at this time, but its description follows.

The Carpentier-Edwards Bioprostheses are comprised of porcine aortic valves which have been preserved in a buffered glutaraldehyde solution and fitted on a flexible frame. Specifically, the frame has been designed to be flexible at the orifice as well as at the commissures. The metal used on the frame is Elgiloy (Elgiloy Corporation), a corrosionresistant alloy of cobalt and nickel. The metal frame is covered with a porous knitted polytetrafluoroethylene cloth to facilitate tissue invasion and encapsulation upon implantation. 

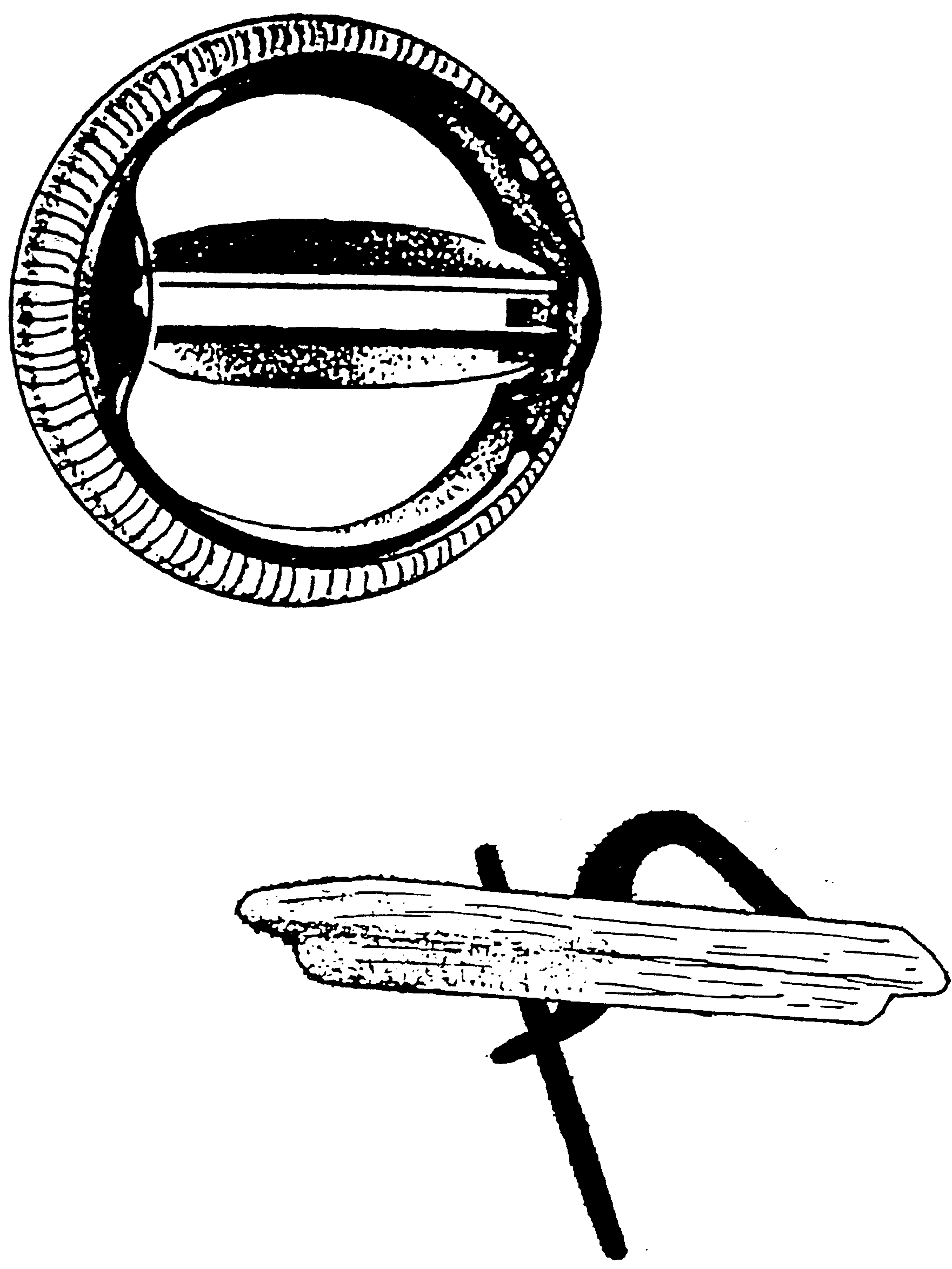

Figure 15: Drawings depict bileaflet (above) and tilting disc (bellow) mechanical prosthetic heart valves. 
Figure sixteen shows a typical pressure plot with each line labeled in order to establish a pattern that can be used with all other graphs; the atrial pressure is always nearly flat, the ventricular oscillates between atrial and aortic and the aortic pressure varies nominally between 80 and $120 \mathrm{mmHg}$. Figures seventeen to thirty-four depict one pressure plot each which is representative of each of 18 sets of experiments. ( 3 valve types, 2 sizes each, under 3 conditions of stenosis each) Notice that on the experiments with stenotic valves, a significantly higher trans-mitral pressure drop is observed. The graphs are labeled with a uniform system that permeates the entire project: two letters (BL, TD, BP), one number $(1,2)$, one letter $(N, M, S)$, the letters 'PS' and one number (1-13). The letters ' $\mathrm{BL}$ ', 'TD' or ' $\mathrm{BP}$ ' indicate the type of valve in question, which can be of the bileaflet, tilting disc or bio prosthetic type, respectively. The numbers ' 1 ' or ' 2 ' reflect the size of the valve in question. ' 1 ' represents the small $(27 \mathrm{~mm})$ and ' 2 ' the large (29 $\mathrm{mm}$ ) of each of the three valve types. The letters 'PS' simply reflect the generic name of all input flow functions, while the numbers 113 reflect the specific function used for that study; the operational parameters of each of which can be seen on table 1 , on page 40

The time axis of each graph is determined by the 
frequency of data acquisition and the number of data points acquired for a particular experiment, which was a nominal 80 to 90 points, but varied slightly from one experiment to another. The onset of systolic action is flagged in the data stream, as explained before; further confirmation of the accuracy of the pressure plots is afforded by the knowledge of the systolic fraction (table 1, page 40) and depicted in the graphs by a sudden and sharp drop in ventricular pressure.

Trans-mitral pressure drops were computed in each case, first by the ensemble averaging the data produced from the twenty strokes of data collected; then, by subtracting the ventricular from the atrial pressure while the former is smaller than the latter, for this condition is directly indicative of diastole and the presence of mitral flow. The mean pressure drop during diastole was computed and its value at the time of peak flow was noted for later use.

Three areas were determined for each valve: the effective area (EA), the mounting area (MA) and the flow area (FA). The EA is the projected open area of the valve, which was measured by the process of photography and planimetry described earlier. The EA is used to generate the Gorlin Coefficient of discharge, which is a measure of the valve's effectiveness while open. The MA is calculated with the nominal size of the valve; this is the area that must be made available on the heart for valve installation. All valves used in this experiment being either 27 or 29 millimeters in diameter, 
produces mounting areas of 5.726 and 6.605 squared centimeters respectively. The Aaslid and Gabbay indexes use this area as a divisor of the orifice area calculated from pressure drop and flow rate measurements to produce a measure of how well the valve uses its mounting area. (Essentially, the effective orifice area is normalized by the mounting area) Finally the FA is the primary flow area of the valve, which is the same as the EA for tissue valves. For mechanical valves, the FA is calculated from its internal diameter, (Both the bileaflet and the tilting disc, also called monoleaflet, valves are circular in internal geometry) which, when used in conjunction with the calculated orifice area, represents a measure of how well the valve uses its primary flow area. Since there are 78 EA or FA values for the bio prosthetic valve group, the result of these measurements is displayed in graphic form in figure thirtyfive. The areas for the mechanical valves are summarized in table two.

As explained before, there are three different flow rates used in association with the four equations for performance parameters, the peak diastolic flow, the mean and RMS flows during diastole. These quantities were calculated from the input flow functions since these were shown earlier to correspond to actual valvular flow. Table three lists the calculated flow rates used to determine each parameter.

The last factor needed for the computation of the performance parameters is the constant. A thorough explanation 
of the origin of the constants in the effective area equations was presented earlier; the calculation of their values only appears here. For the Gorlin and the Gabbay equations, the units of flow rate must be cubic centimeter per second and the those of pressure drop, millimeter of mercury. Dimensional equality-demands that the pressure drop be converted to centimeters of fluid first, then the constant becomes:

$\frac{\mathrm{mm} \text { of } \mathrm{Hg} \times 13.55}{10 \times 1.093}=\mathrm{cm}$ of fluid $=1.2412$

where 13.55 is the density of mercury $\left(\mathrm{g} / \mathrm{cm}^{3}\right)$

1.093 is the density of the fluid used.

$$
\begin{gathered}
C=\sqrt{(2 \times g \times 1.2412)}=49.3233 \\
\text { where } g=980 \mathrm{~cm} / \mathrm{sec}^{2}
\end{gathered}
$$

For the Aaslid formula the flow is inserted in the units of liters per minute, hence the Aaslid constant of 0.3379 is simply the division of 16.667 (1000/60, lit/min into $\mathrm{cm}$ ^ $3 / \mathrm{sec})$ by the same constant described above.

$$
\text { Aaslid constant }=\frac{16.6667}{49.3233}=0.3379
$$

Although dimensional requirements demand that 'flow rate' be used to calculate the effective orifice area by each of the four equations, correct interpretation of the results require 
that they be analyzed and studied as a function of the dimensionless Reynolds number, since the performance parameter itself is dimensionless. The use of the Reynolds Number with highly irregular geometries (such as an intricate heart valve with a central occluder) finds the determination of the characteristic length problematic.

The solution to this problem is implicit in its statement: the reason for defining the performance of valves in terms of four different parameters, is that there is no absolute way of defining what flow rate and area should be used with an EOA formula (for dimensional equality it is only necessary that a flow rate and area be used). Schoephoerster [14], Aaslid [23] and Gabbay [24] have each shown that either the peak or the RMS flow are more appropriate than the mean flow rate, but either of former two are dimensionally correct. The selection of what, out of the three described, area should be used for the normalization of the orifice area is totally arbitrary; the use of each, however, produces a measure of a different aspect of the valve's performance. (More will be said about this later) Calculation of the Reynolds number, then, follows the pattern that each of the previous investigators used, and where each definition of flow rate is used for the $C d$, it is also used for the velocity, by dividing the former by the same area that was used for the calculation of the parameter. The characteristic length is the mounting diameter, in case of the Gabbay performance index and the 
Aaslid effective area index, the same diameter used to determine the mounting area, previously described. For the Gorlin formula the effective area was used; for the 'Gorlin' Reynolds number, the $\mathrm{EA}$ is assumed to be perfectly circular and its equivalent diameter is calculated and used as the characteristic length for the calculation of the Reynolds Number. The primary flow (internal) diameter of each valve is used as the characteristic length for the Gabbay Reynolds number. Table four summarizes the terms used for the calculation of the Reynolds numbers associated with each experiment. Finally, table five lists all four performance parameters calculated for each experiment.

Since, in general, the coefficient of discharge of prosthetic heart valves has been shown to increase with increased flow rate, thus Reynolds number [15], a natural way to start the investigation of the results obtained from these experiments was to lump all parameters together without respect to valve type, size or condition, and plot them with respect to flow rate. If the performance parameters described were to vary only with the Reynolds number, regression analysis could be carried out on the population of data points and a best-fit curve generated. If that were to happen this project would end, but instead, it is observed on figures thirty-six and thirty-seven that the spread of values is so wide that no reasonable conclusion can be drawn. The reason for such disparity on the data population can be caused by 
several facts: incorrect measurements and calculations; the established fact that the performance parameters are different for the different valvular configurations used; or lastly, the method does not work for performance assessment of heart valves because no correlation for the $\mathrm{Cd}$ can be established that applies for any condition of stenoticity.

Deeper investigation of the performance of these valves is carried out in a systematic fashion in order for true trends to be revealed. At this point, the results obtained will be simply presented; a complete examination and interpretation of such results is carried out in the next section of this paper. It can be prematurely concluded, however, that a performance parameter which is a function of the Reynolds number only would not be adequate to assess the condition of these valves.

A simple Poiseuille flow will show a pressure drop that is linearly dependent on Reynolds number, more complex flows will produce pressure losses that are not linearly related to Reynolds number; the exact configuration of this curve is indicative of the type of flow under observation. Therefore, the first line of investigation carried out seeks to determine what is the basic characteristic of the flow through the valves by means of graphical analysis of the pressure drop through the valve as a function of the Reynolds number. As each plot of the data population is produced, polynomial regression analysis is carried out with successively higher 
order, until the correlation coefficient either no longer increases or actually decreases. Figures 38,39 and 40 show such plots with the regression polynomial depicted. It is observed that excellent fit with a second order polynomial is attained. This validates the data obtained, for it is theoretically correct and in agreement with the results of other investigators.

Next, each of the four performance parameters described is plotted against each of the three dimensionless numbers that describe similitude with the physiological system under investigation, these are the Reynolds, Womersley and strouhal numbers. Both the Womersley and the strouhal numbers are a measure of the pulsatility of the system; the former represents the ratio of transient (frequency dependent) inertial to viscous forces, while the latter relates transient inertial to convective forces. The Reynolds number represents the ratio of inertial to viscous forces. The relevance and interpretation of each of these plots will be fully explained in the next section of this work. Figures 41-47 show the plots herein described.

Since the opening area (EA or FA) of the tissue valves assumes a different value each time it opens, unlike the mechanical valves which always open fully early in diastole and remain so for its duration, it was speculated that the this variation was related to one or more of the dimensionless parameters used to model the system. Flow areas for each of 
the 78 experiments with tissue valve were then plotted as a function of the Reynolds, Womersley and Strouhal numbers; such plots can be observed on figure 48 .

Lastly, knowing a priori what magnitudes of power are produced in the human cardiovascular system, namely, approximately 1.5 Watts of power (a pressure increase of 120 mmHg, on a volume of $80 \mathrm{ml}, 72$ times a minute) are imparted onto the blood every cardiac stroke, a quick indication of the relevance of these experiments to the physiological situation can be attained. Where simulated valvular power losses approach the tens of Watts, clearly, the results obtained are of academic relevance only. For this purpose, the mean pressure drop was multiplied by the mean flow rate in order to obtain an estimation of the actual power dissipated by the valve, which was then plotted as a function of the Reynolds number and is depicted in figure 49. 


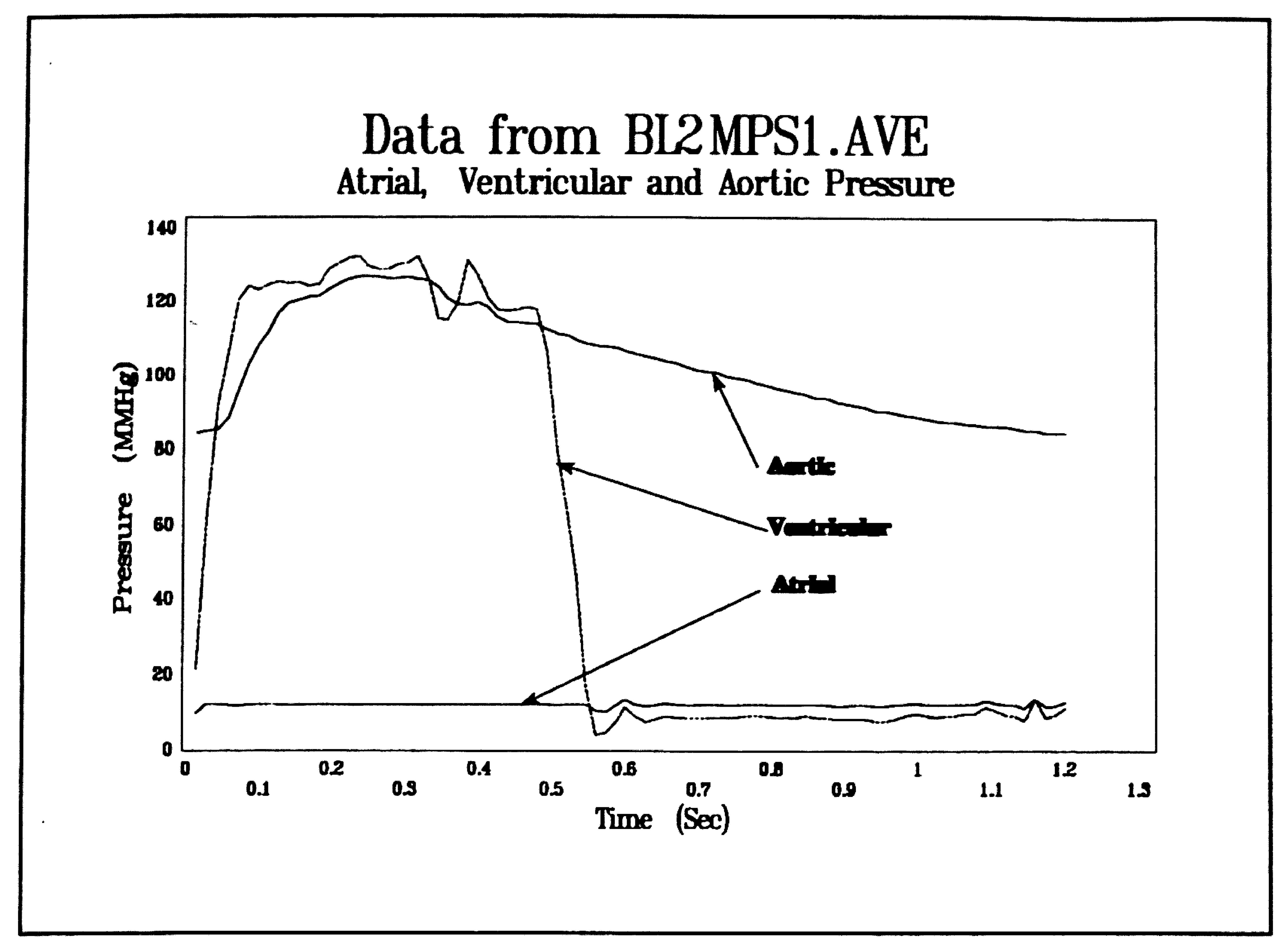

Figure 16: Typical graphic output of experiments. Aortic, ventricular and Atrial pressures are seen clearly labeled. 


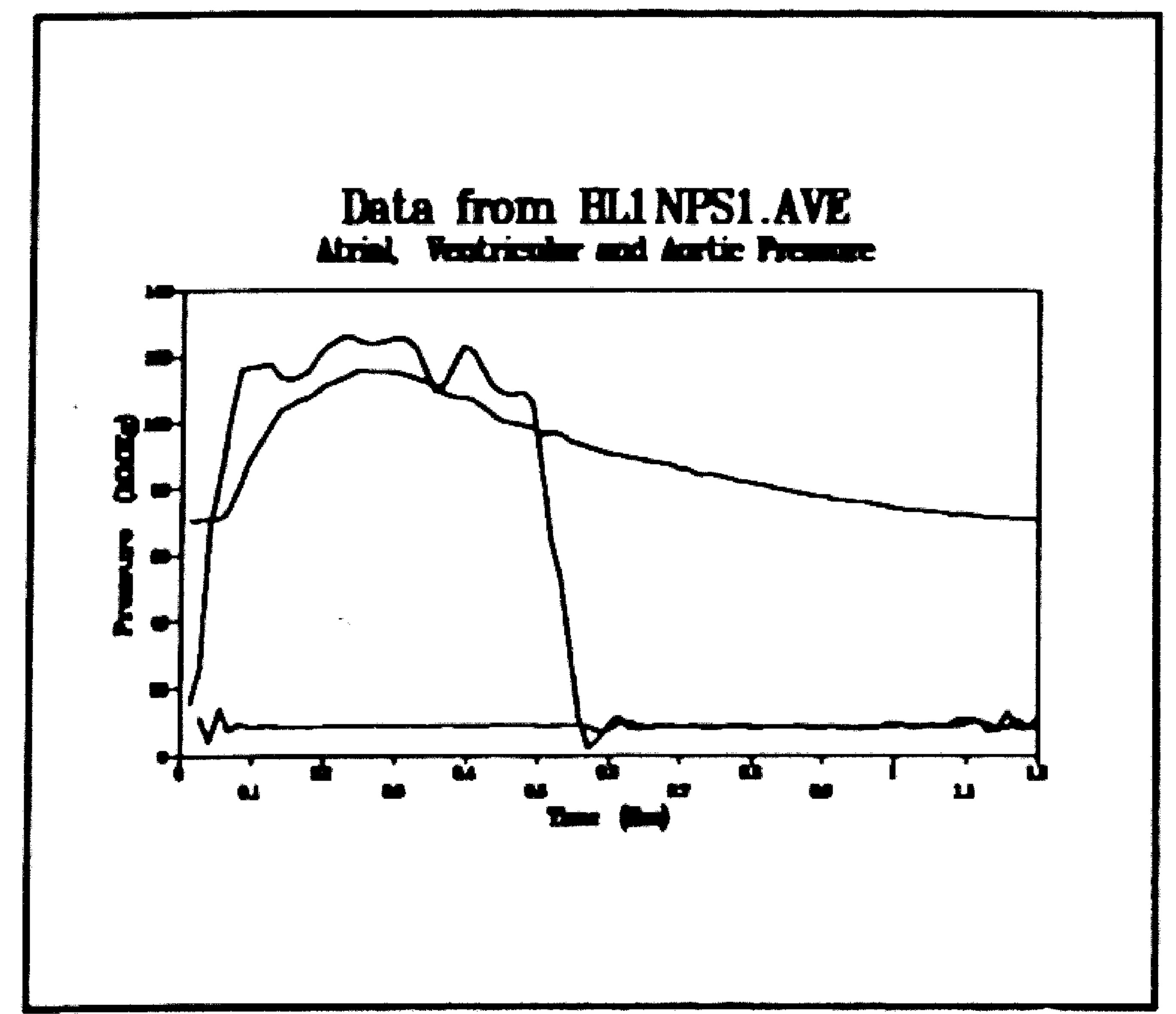

Figure 17

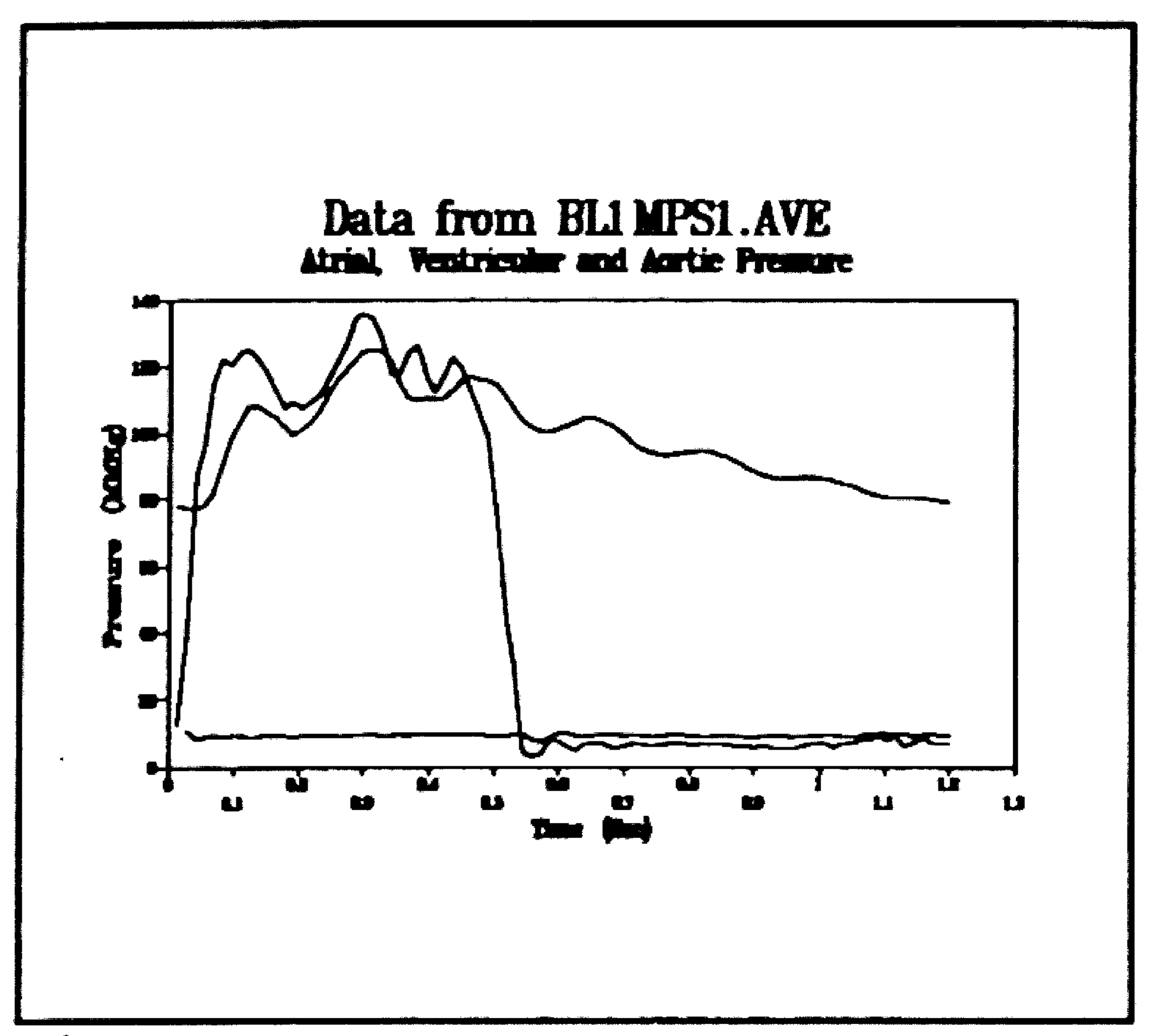

Figure 19

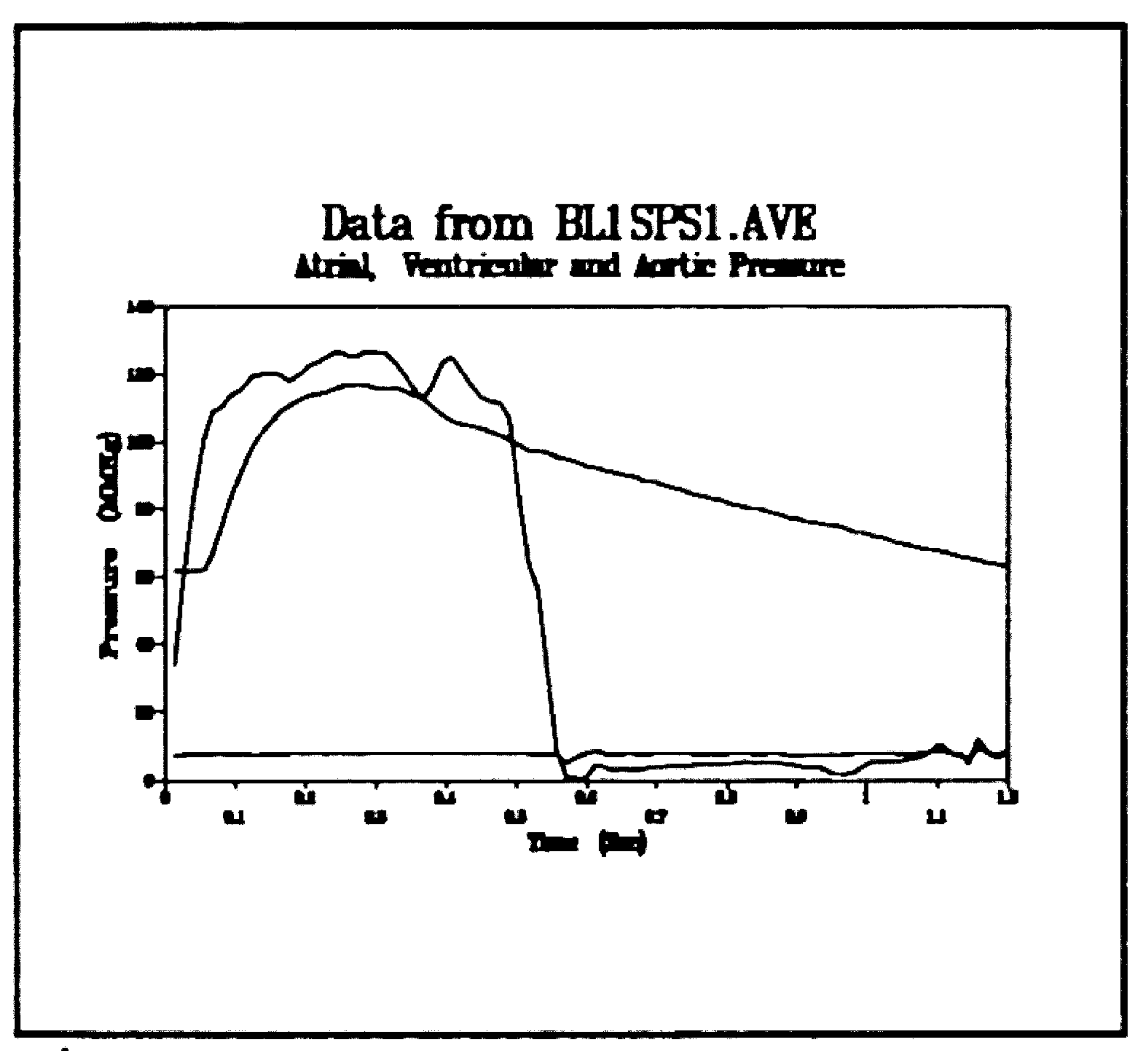

Pigure 21

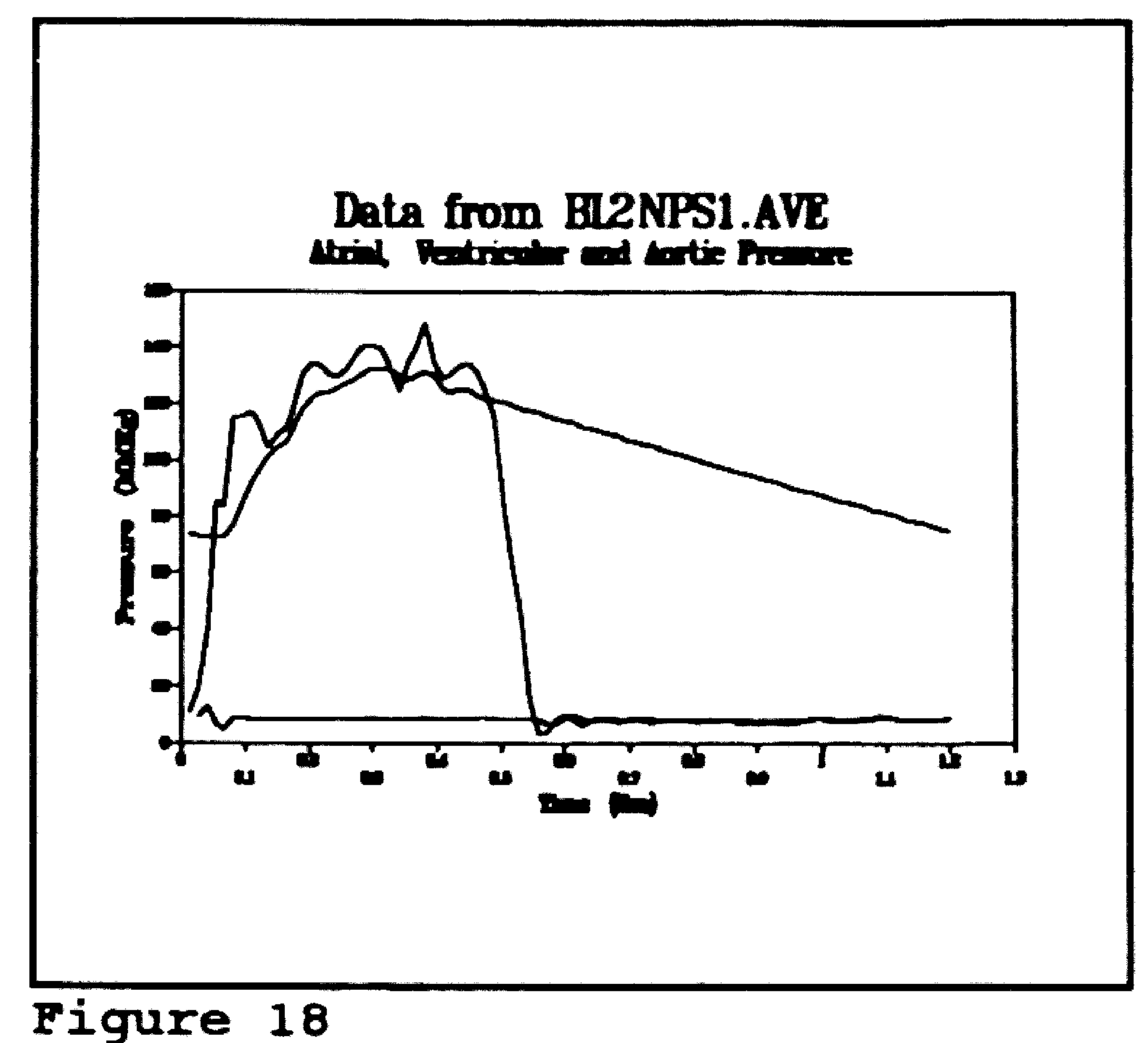

Figure 18

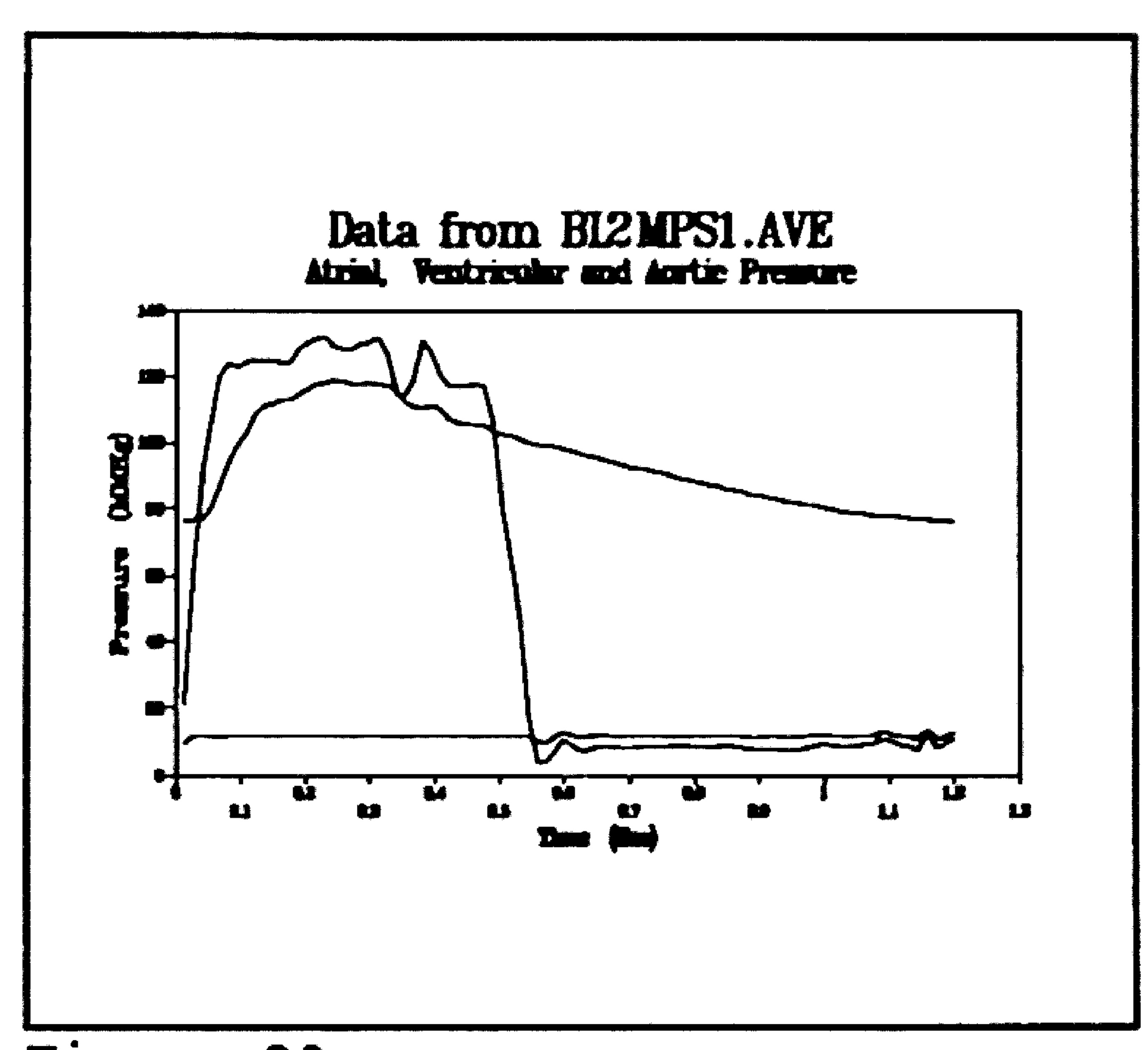

Figure 20

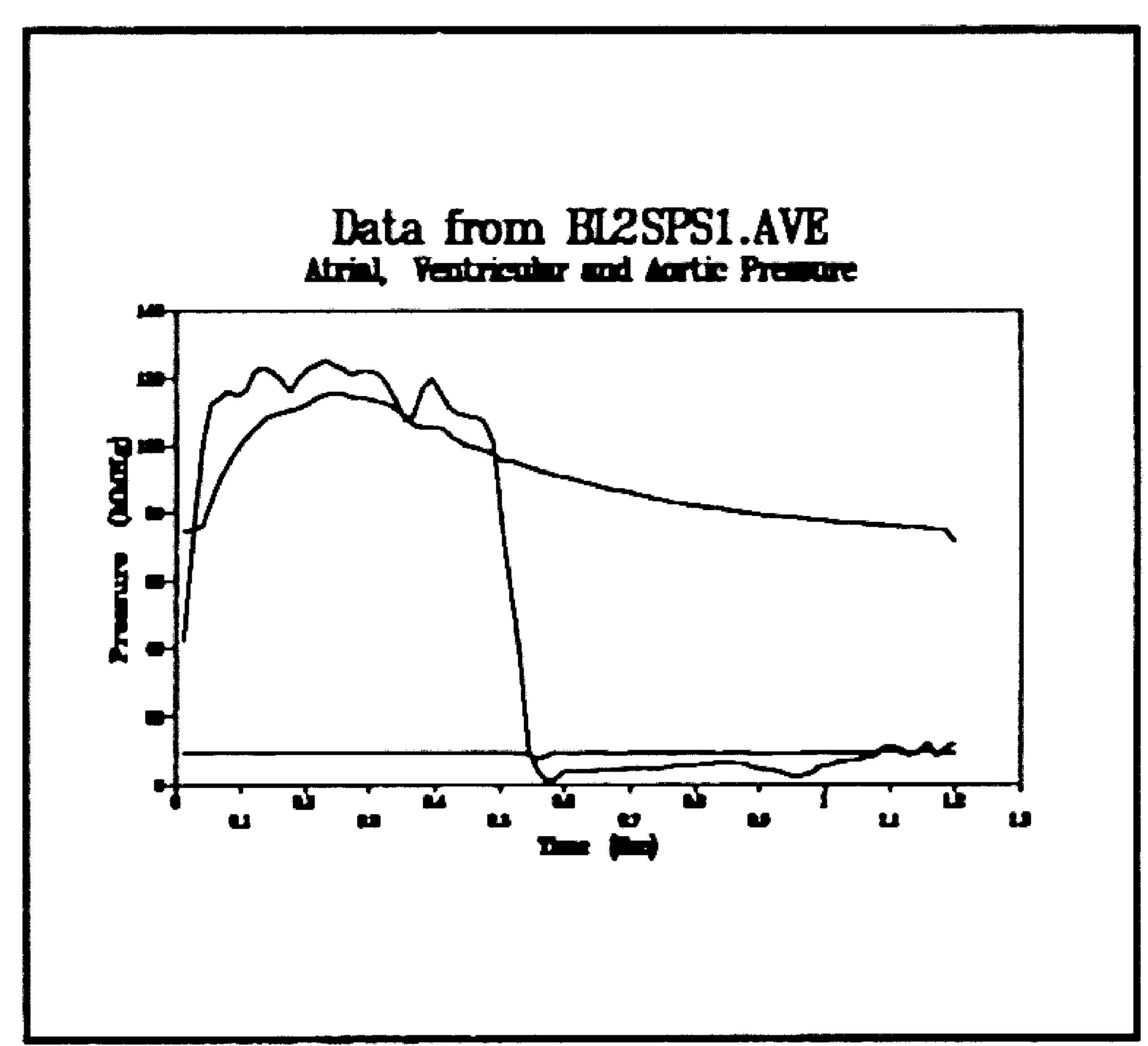

Figure 22 


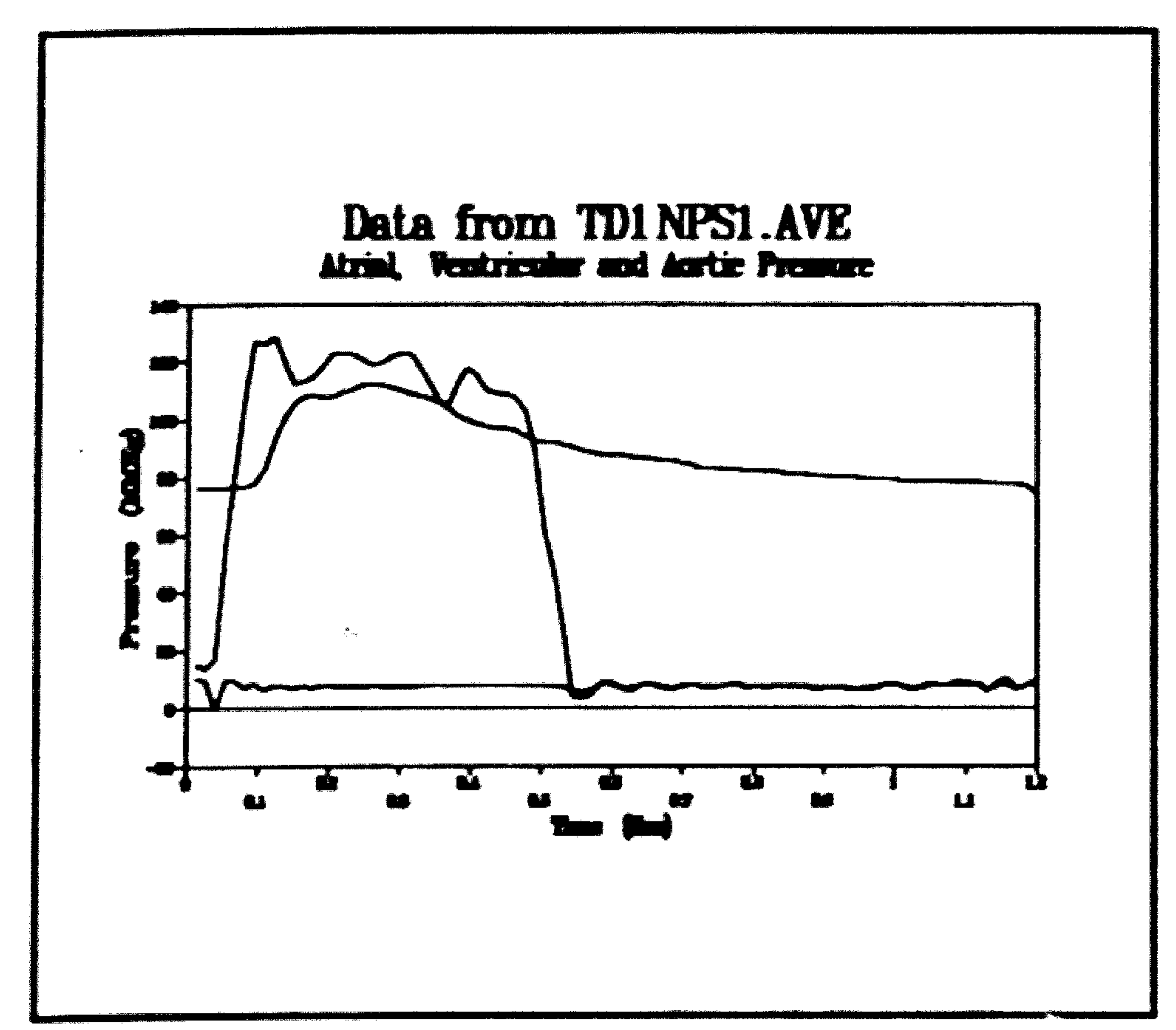

Figure 23

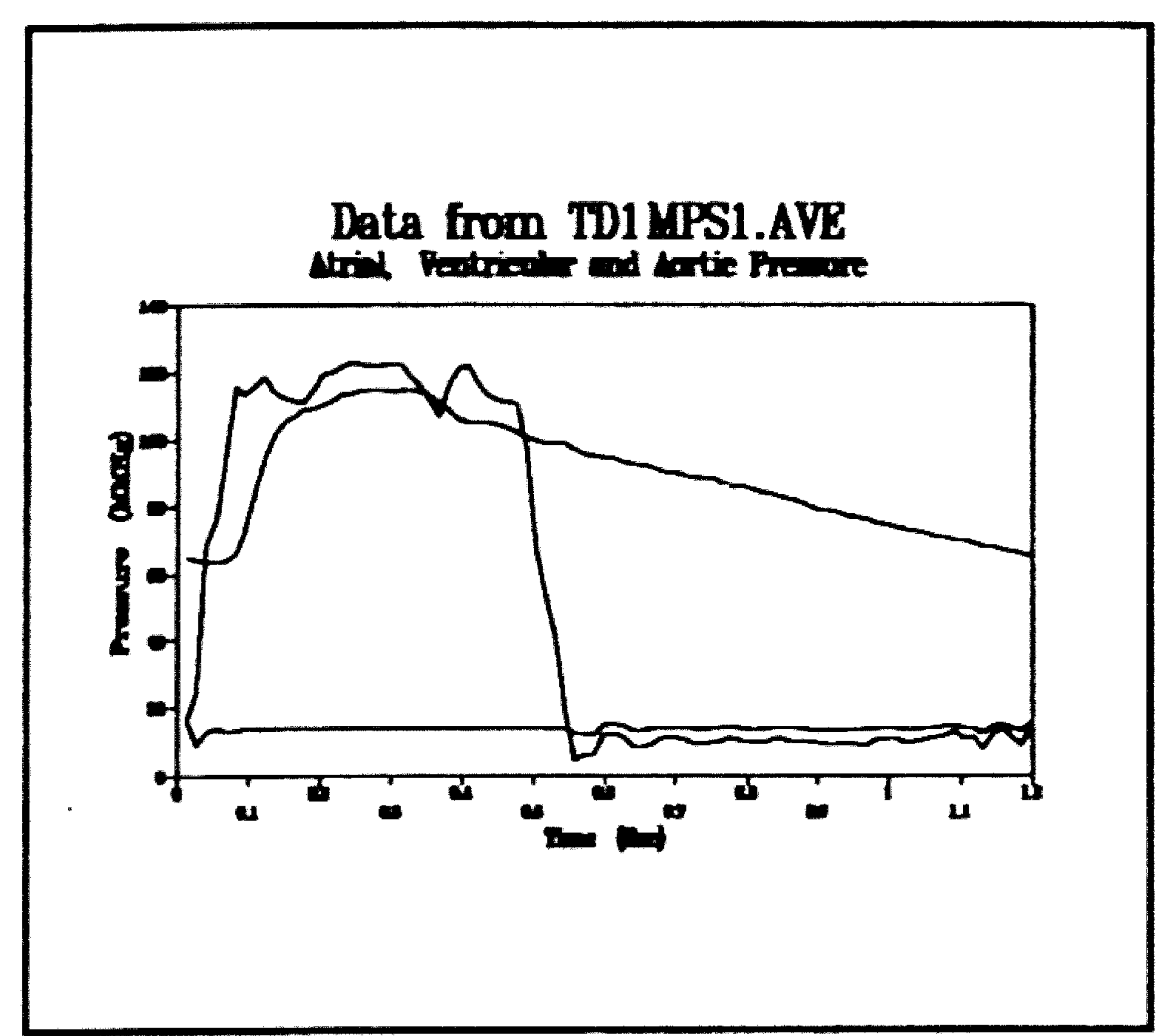

Figure 25

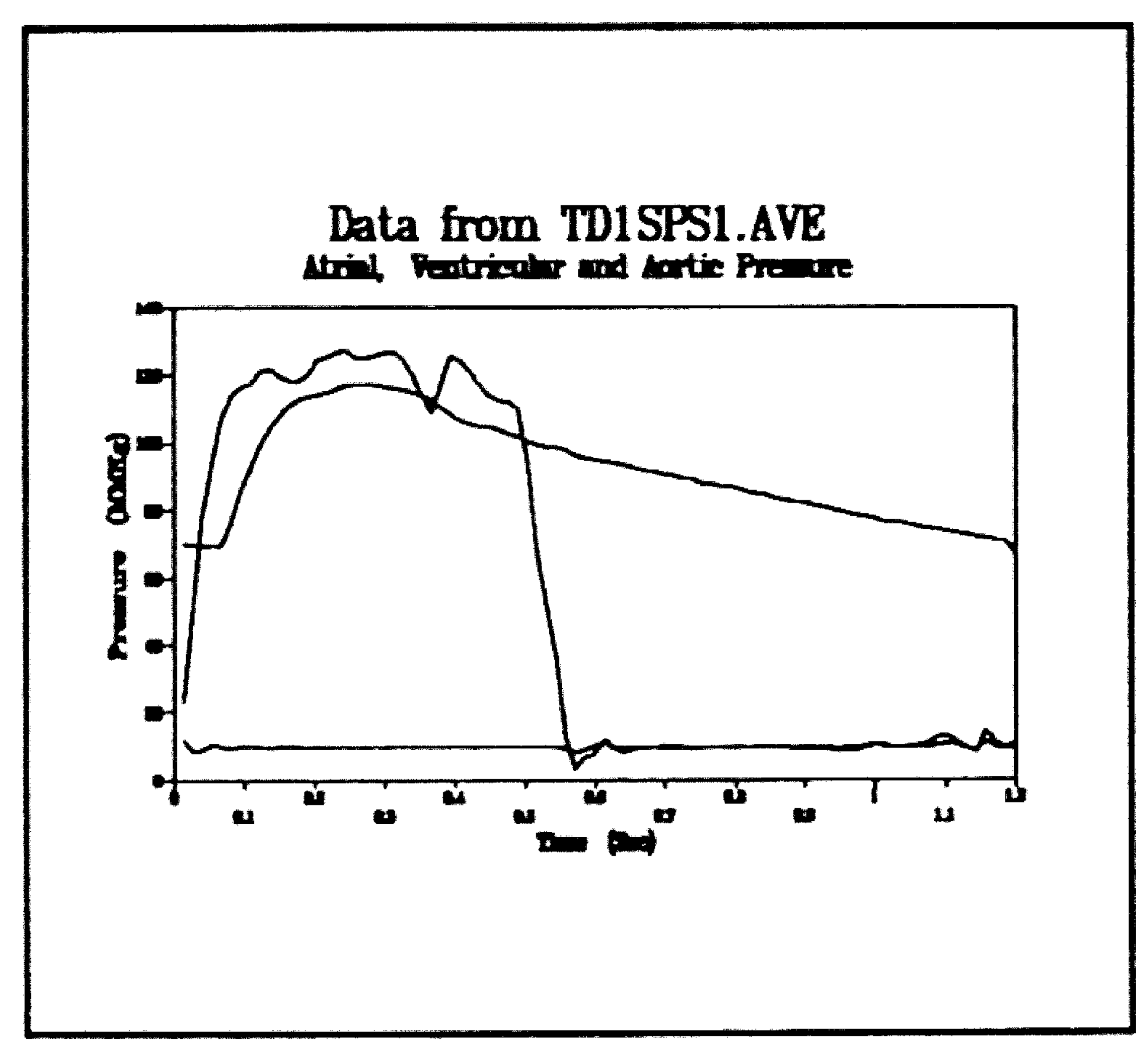

Figure 27

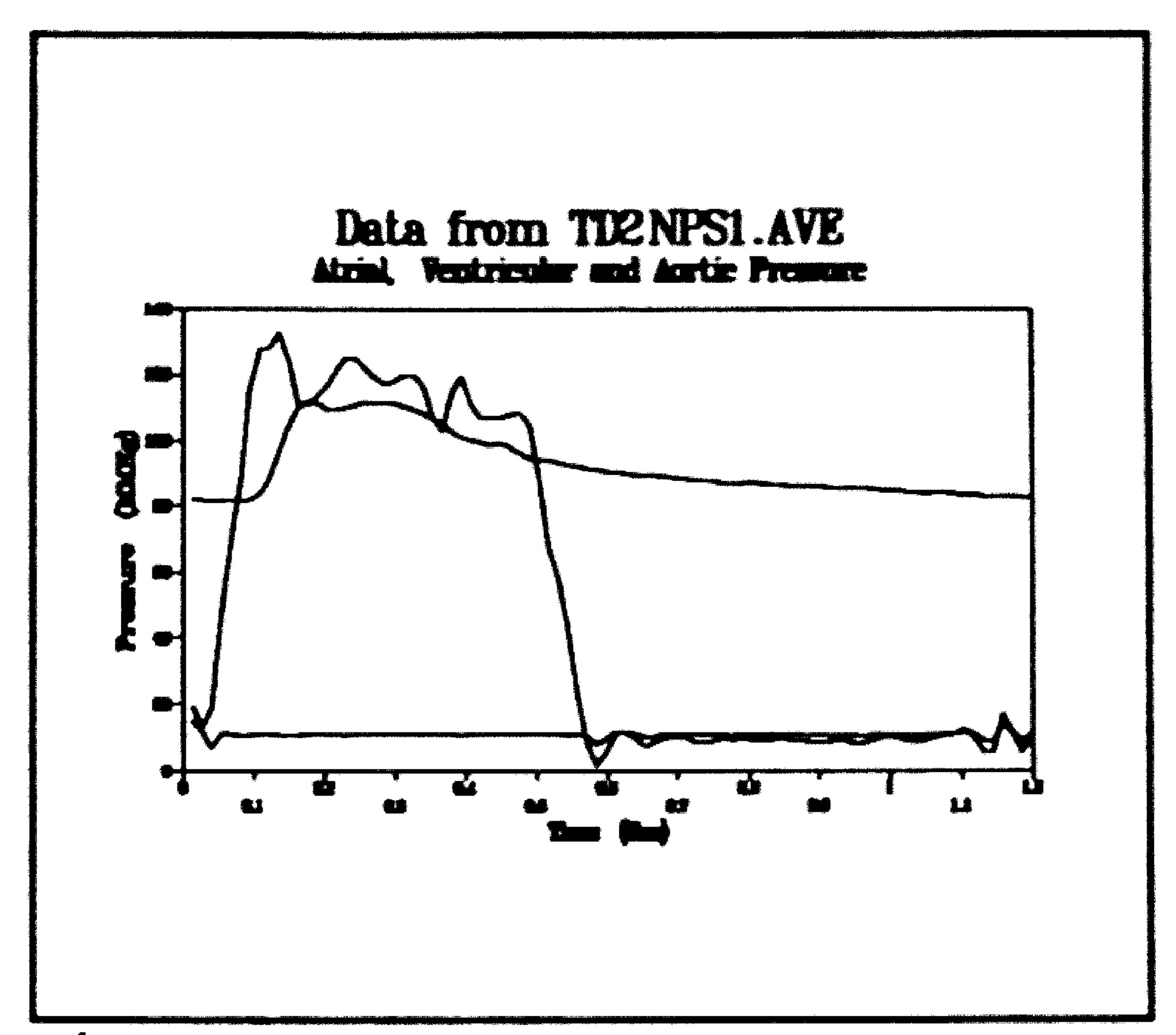

Figure 24

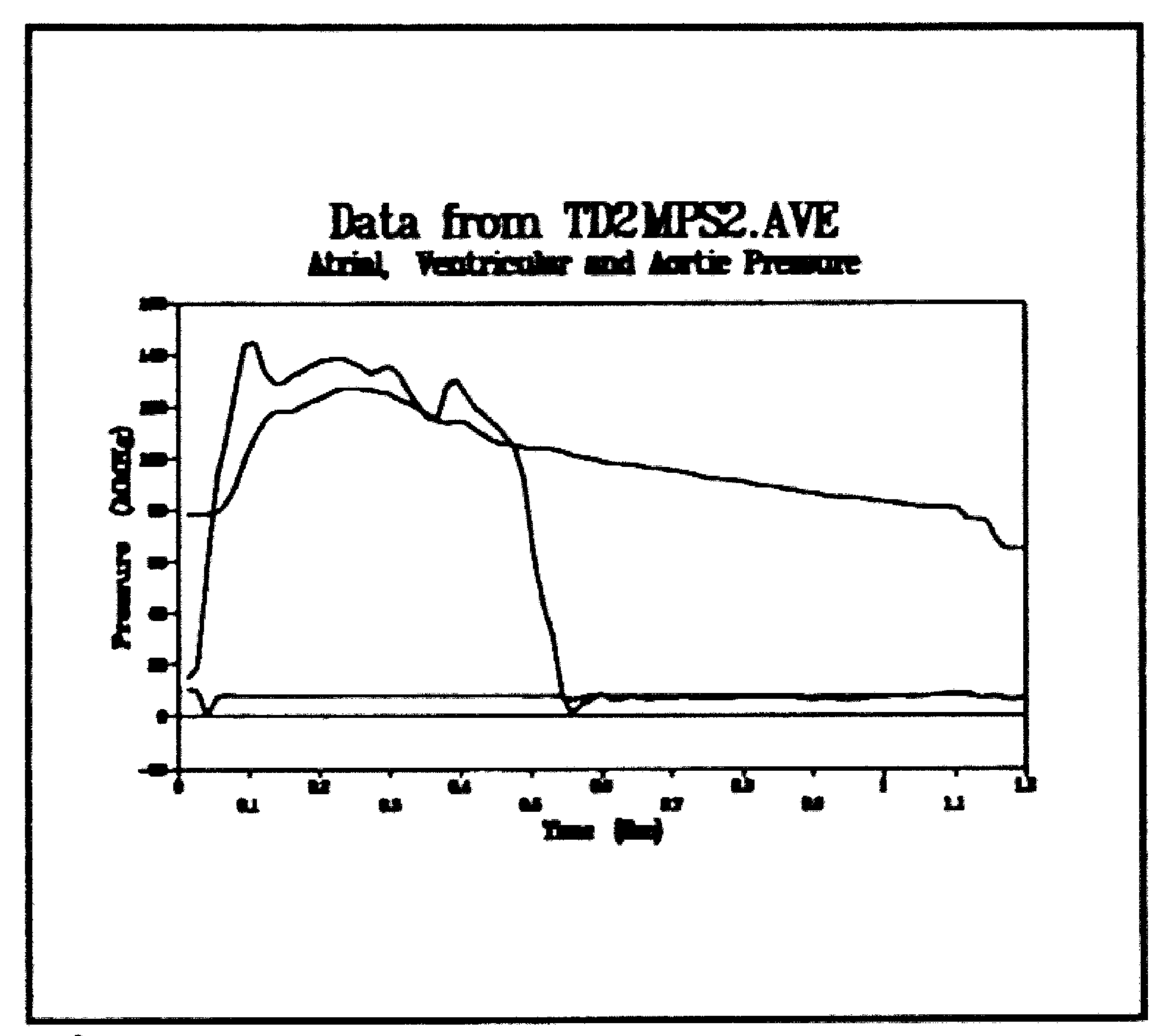

Figure 26

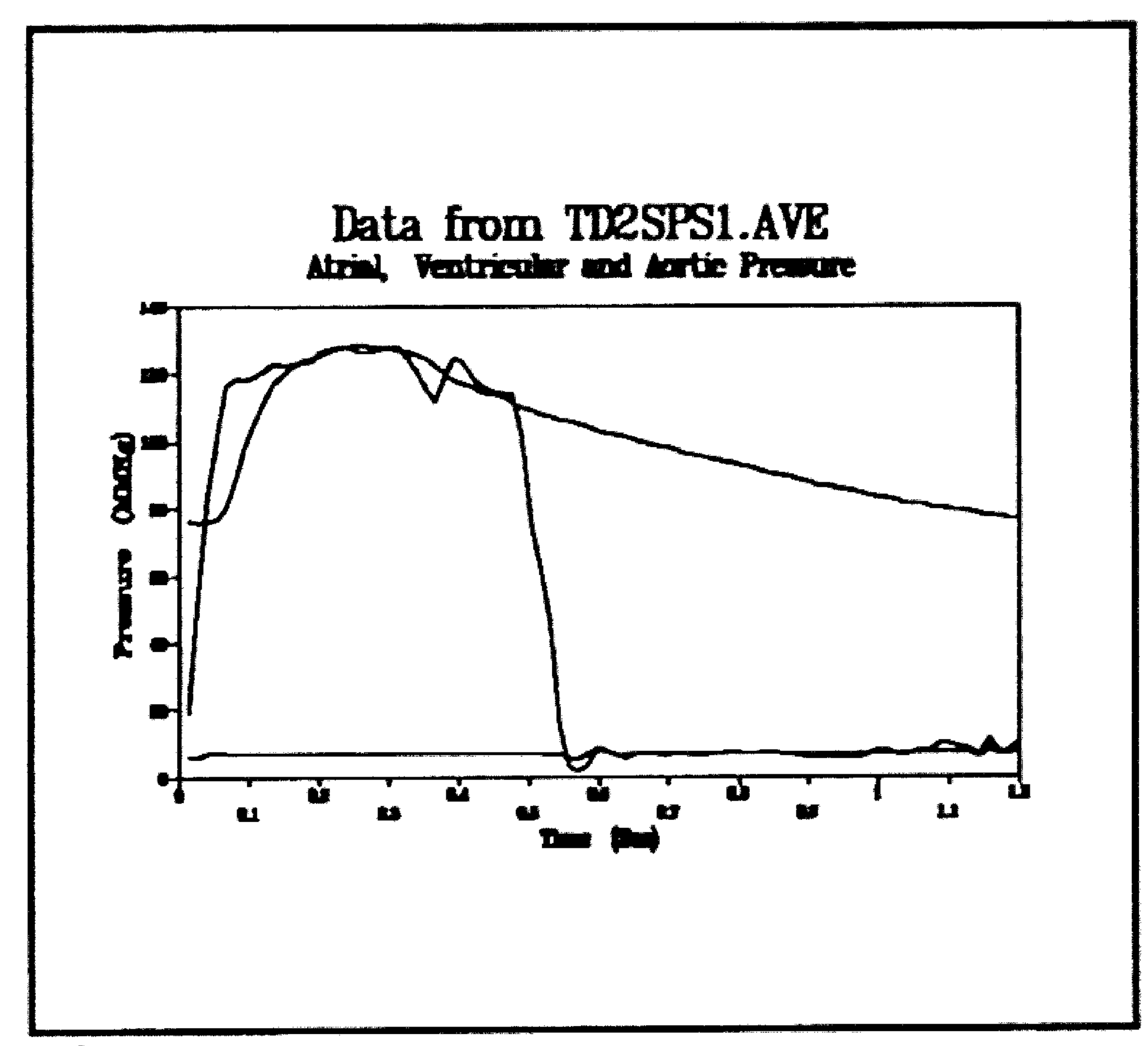

Figure 28 


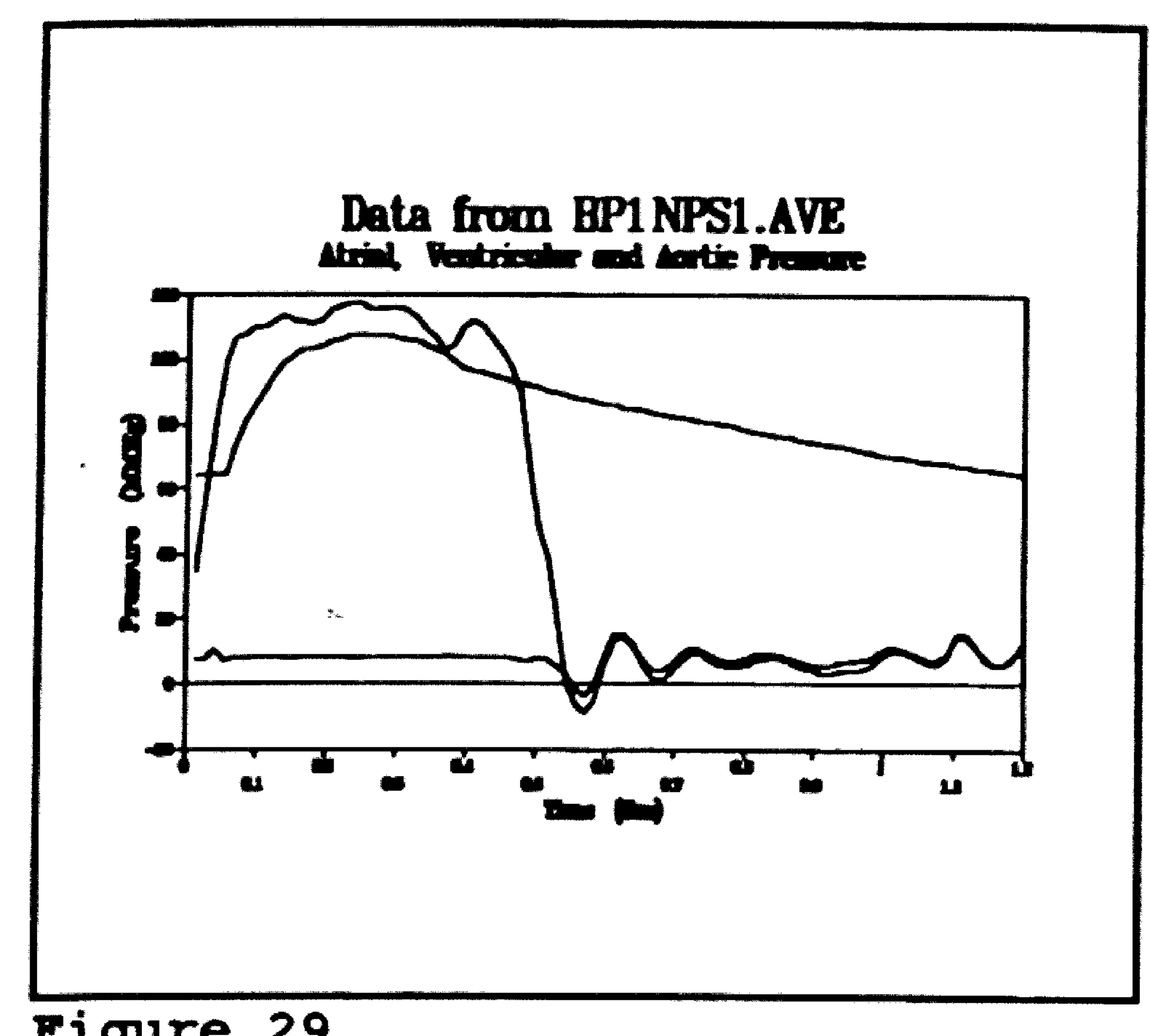

Figure 29

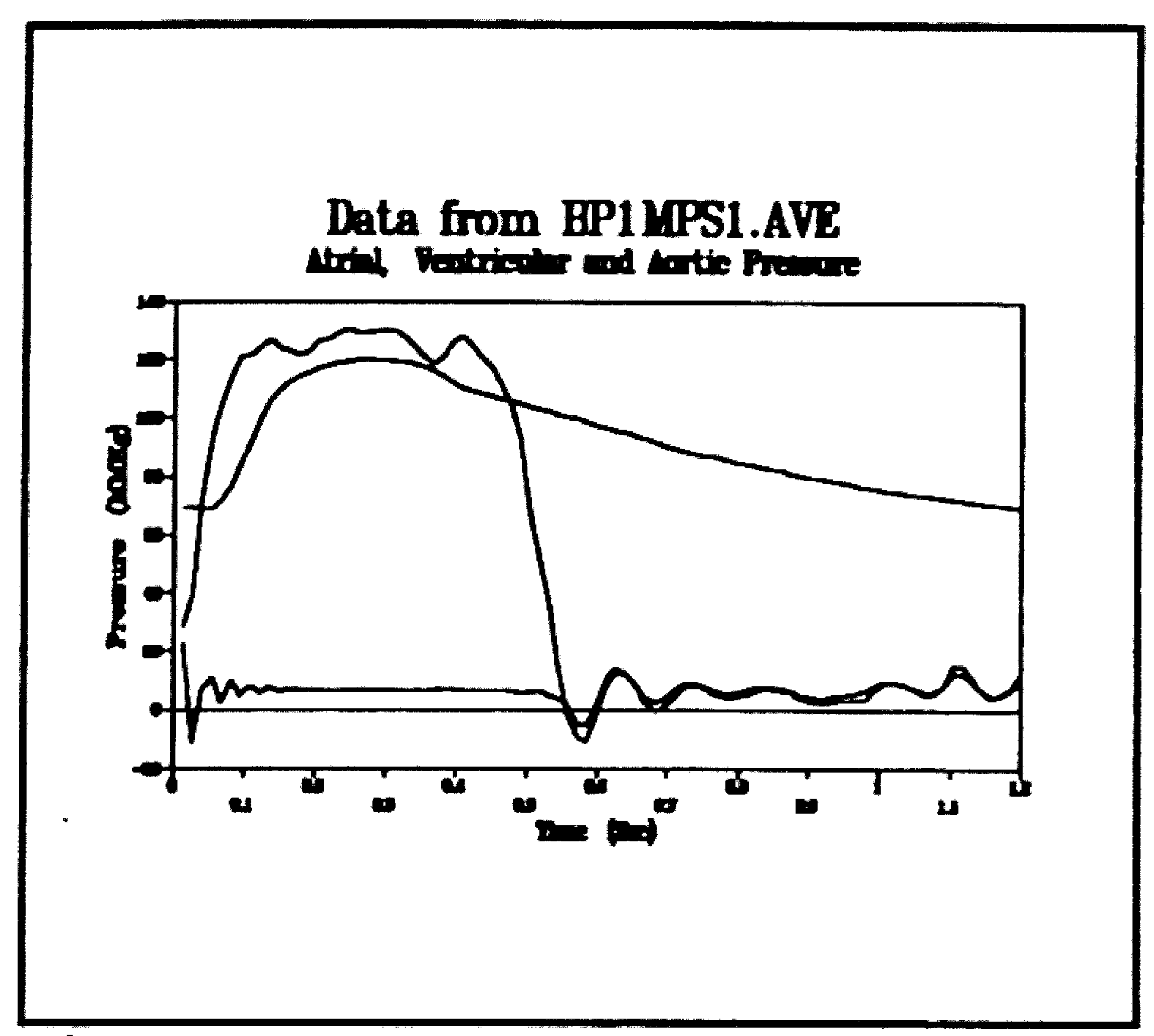

Figure 31

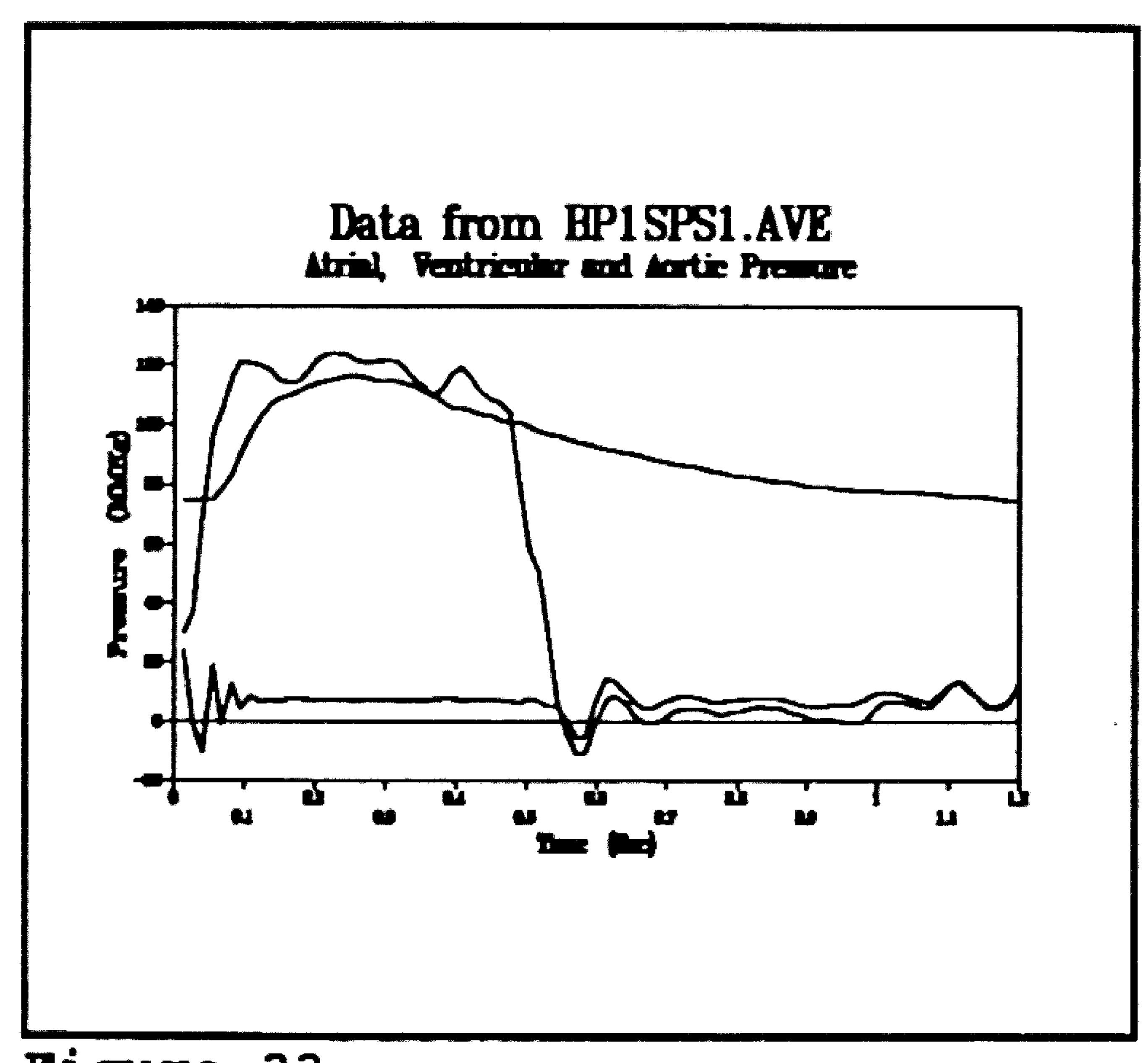

Figure 33

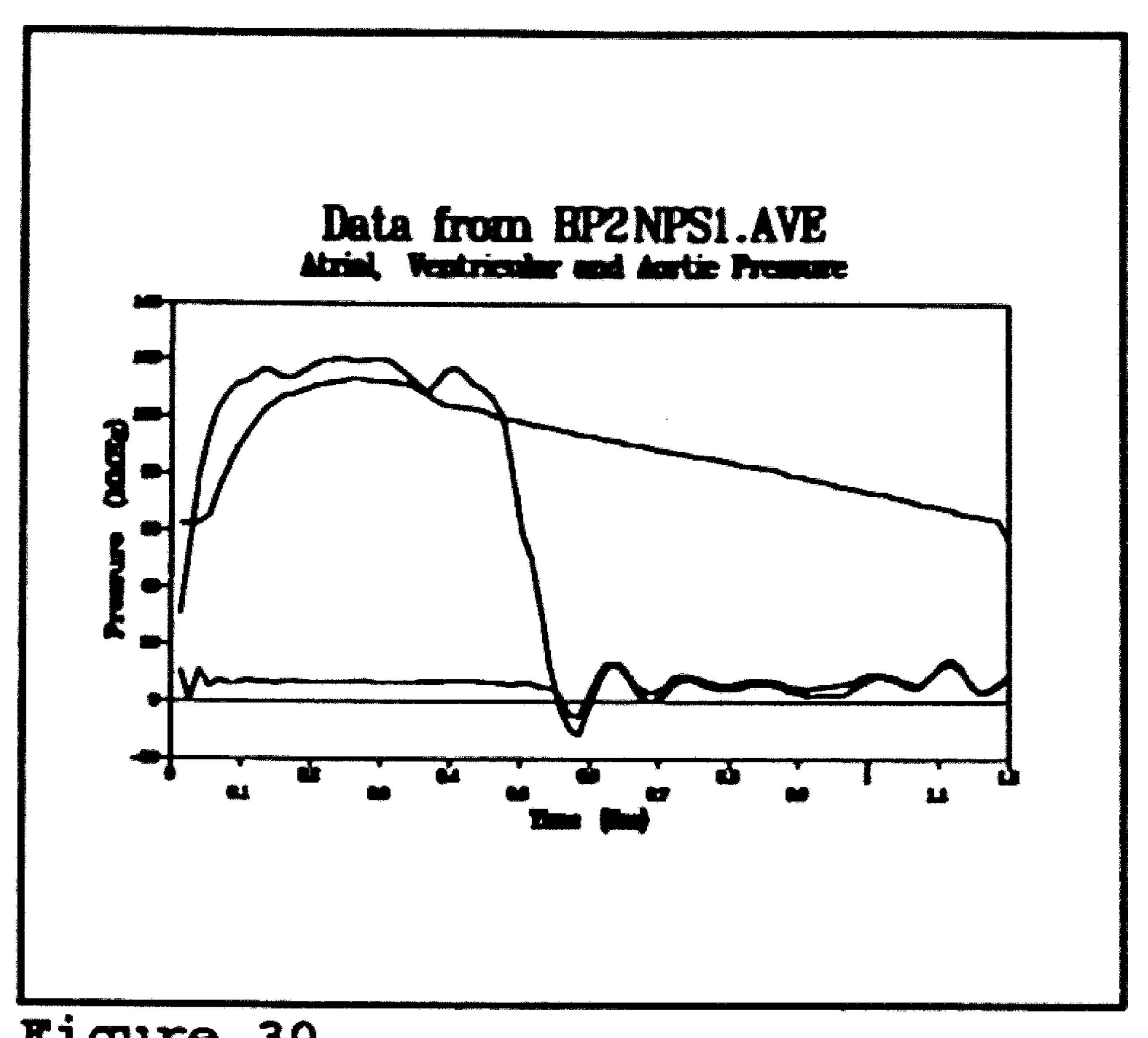

Figure 30

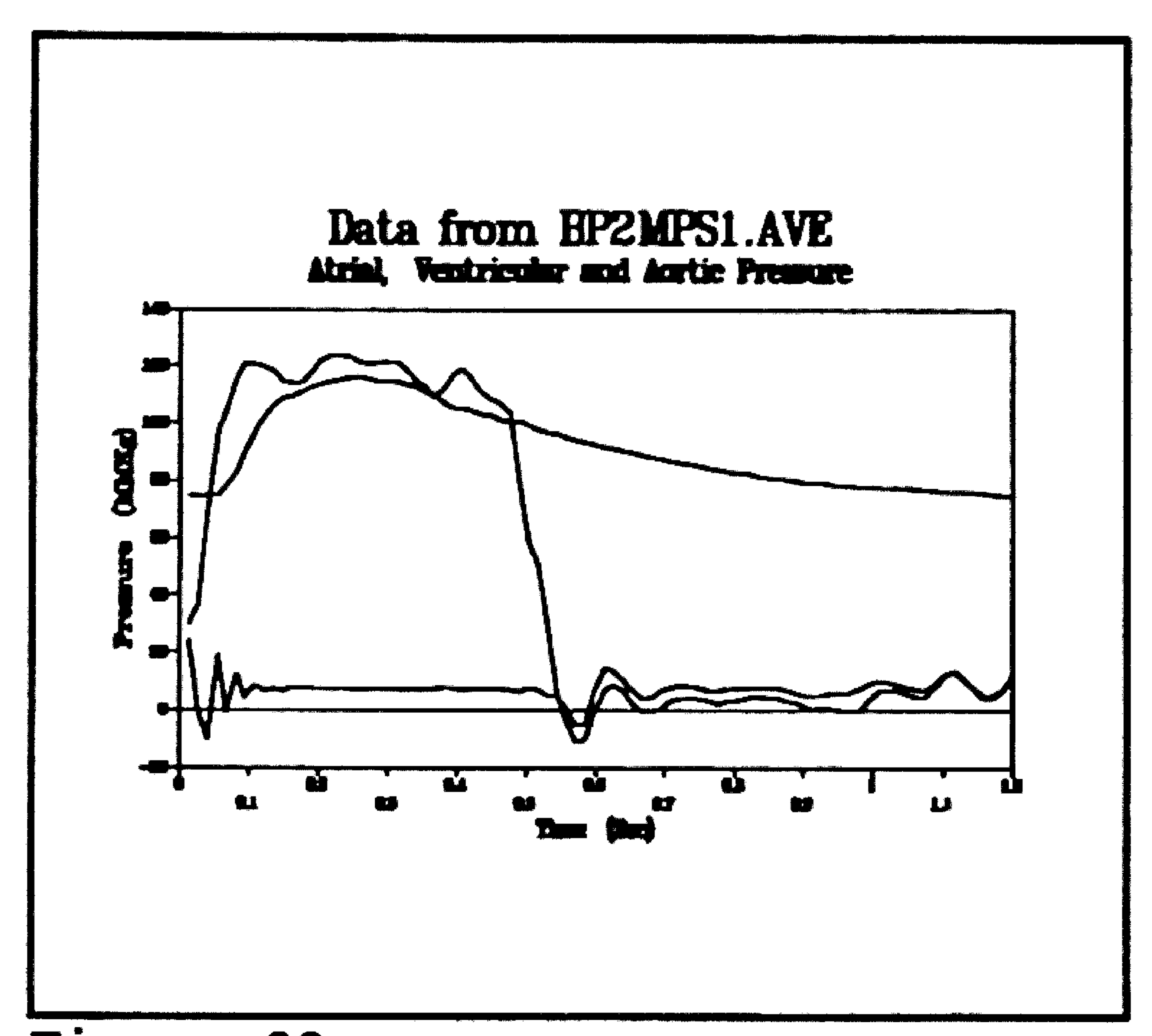

Figure 32

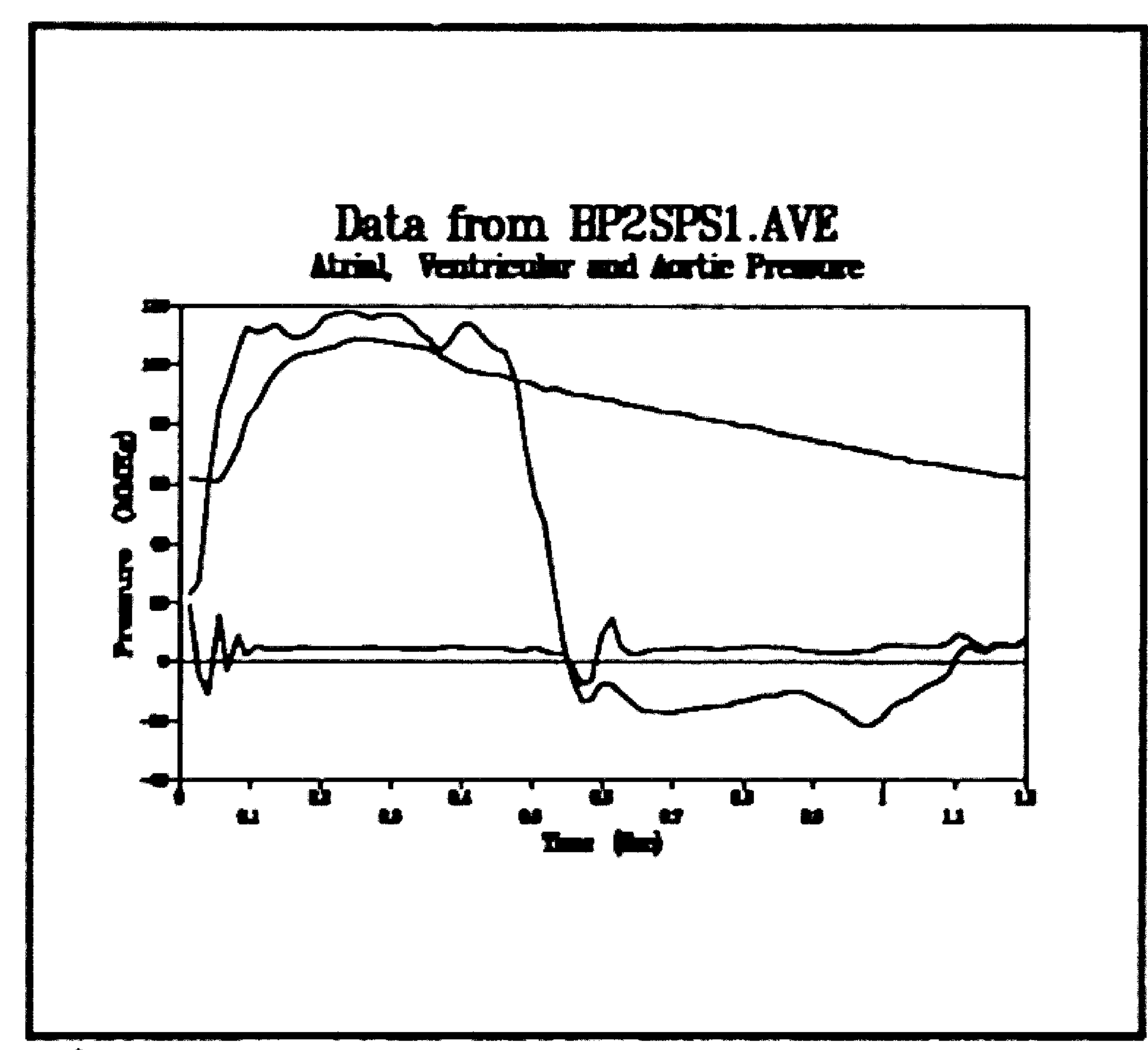

Figure 34 


\section{Measured Effective Areas - Tissue Valve \\ Normal, Mild and Severe Stenoticity}

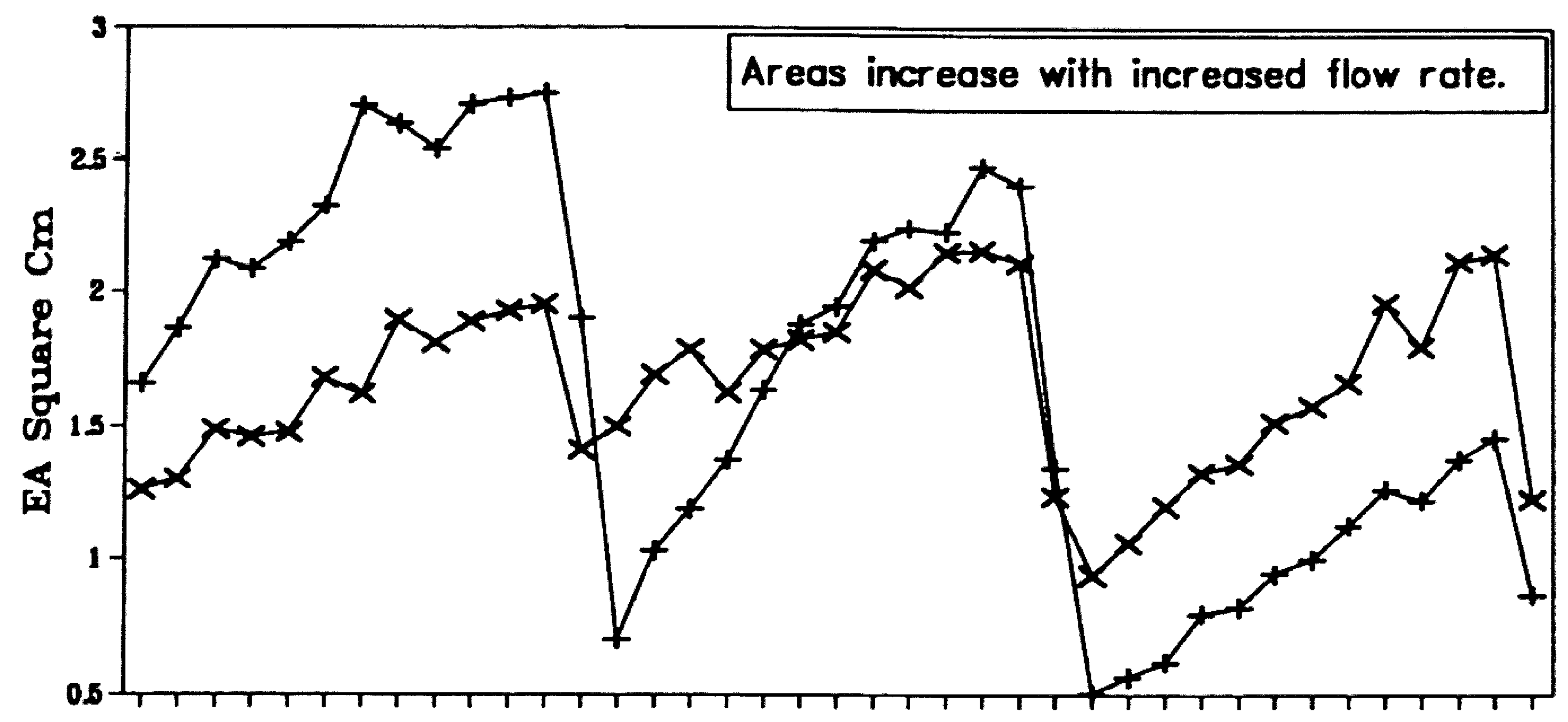

Experiment

$\times 27 \mathrm{~mm}+29 \mathrm{~mm}$

Figure 35: Effective areas for 78 experiments with tissue valves. Carpentier-Edwards porcine valves in 27 and $29 \mathrm{~mm}$ sizes were used. Each of the three humps on the curve represent one degree of stenoticity, with the normal valve shown on the left, the mildly stenotic in the middle and the severely stenotic on $t h e$ right. 
SUMMARY Of AREAS fOI MECBANICAL VALVES

$\begin{array}{llll}\text { VALVE TP. } & \text { EA }(C m 2) & \text { FA }(C m 2) & M A(C m 2) \\ \text { BL1N } & 3.254 & 3.942 & 5.726 \\ \text { BL2N } & 3.706 & 4.592 & 6.605 \\ \text { BL1M } & 1.924 & 3.942 & 5.726 \\ \text { BL2M } & 1.775 & 4.592 & 6.605 \\ \text { BL1S } & 0.935 & 3.942 & 5.726 \\ \text { BL2S } & 0.871 & 4.592 & 6.605 \\ \text { TD1N } & 1.791 & 3.748 & 5.726 \\ \text { TD2N } & 2.406 & 4.525 & 6.605 \\ \text { TD1M } & 1.187 & 3.748 & 5.726 \\ \text { TD2M } & 1.278 & 4.525 & 6.605 \\ \text { TD1S } & 0.623 & 3.748 & 5.726 \\ \text { TD2S } & 0.794 & 4.525 & 6.605\end{array}$

Table 2: Summary of three types of areas for Bileaflet and tilting disc mechanical heart valves. 
MITRAL FLOW RATES (Liter/Minute)

$\begin{array}{llll}\text { INPUT } & \text { Mean } & \text { RMS } & \text { Peak } \\ \text { PS1 } & 5.606 & 5.759 & 7.501 \\ \text { PS2 } & 7.606 & 7.603 & 10.100 \\ \text { PS3 } & 9.633 & 9.884 & 12.707 \\ \text { PS4 } & 10.374 & 10.651 & 13.688 \\ \text { PS5 } & 14.032 & 14.381 & 18.265 \\ \text { PS6 } & 17.631 & 18.048 & 22.679 \\ \text { PS7 } & 17.533 & 18.972 & 21.383 \\ \text { PS8 } & 28.777 & 27.098 & 31.682 \\ \text { PS9 } & 31.290 & 31.892 & 38.563 \\ \text { PS10 } & 31.018 & 31.062 & 38.279 \\ \text { PS11 } & 41.207 & 41.875 & 49.216 \\ \text { PS12 } & 36.299 & 36.935 & 44.023 \\ \text { PS13 } & 7.142 & 7.267 & 8.674\end{array}$

Table 3: Summary of flow rates used for calculation of performance parameters. 
Table 4: The four possible 'types' of reynolds numbers are displayed.

Esperiment:
Gorlin

Aasid
Gabbay

1412

1864

2424

2612

3526

4426

4652

6645

7820

7617

10268

9057

1782

1315

1736

2257

2432

3283

4120

4331

6187

7281

7092

9560

8432

1659

$\begin{array}{rr}1064 & 10051 \\ 2177 & 1980\end{array}$

2371

3218

4075

4388

5936

7458

7417

12173

13236

13121

17432

15355

3021
1839

2477

3116

3357

4479

5561

5243

7769

9456

9387

12069

10795

2127
1412

1864

2424

2612

3526

4426

4652

6645

7820

7617

10268

9057

1782 
Table 4: The four possible 'types' of reynolds numbers are displayed.

$\begin{array}{lrrrr}\text { Erperiment: } & \text { Gorlin } & \text { Anslid } & \text { Gabbay } & \text { Gabbay } \\ \text { BL2MPS1 } & 2469 & 1713 & 1577 & 1315 \\ \text { BL2MPS2 } & 3350 & 2306 & 2082 & 1736 \\ \text { BL2MPS3 } & 4243 & 2901 & 2706 & 2257 \\ \text { BL2MPS4 } & 4569 & 3125 & 2916 & 2432 \\ \text { BL2MPS5 } & 6180 & 4170 & 3938 & 3283 \\ \text { BL2MPS6 } & 7765 & 5178 & 4942 & 4120 \\ \text { BL2MPS7 } & 7722 & 4882 & 5195 & 4331 \\ \text { BL2MPS8 } & 12674 & 7233 & 7420 & 6187 \\ \text { BL2MPS9 } & 13781 & 8804 & 8732 & 7281 \\ \text { BL2MPS10 } & 13661 & 8739 & 8505 & 7092 \\ \text { BL2MPS11 } & 18149 & 11236 & 11466 & 9560 \\ \text { BL2MPS12 } & 15987 & 10051 & 10113 & 8432 \\ \text { BL2MPS13 } & 3146 & 1980 & 1990 & 1659 \\ \text { BL1SPS1 } & 3402 & 1839 & 1702 & 1412 \\ \text { BL1SPS2 } & 4616 & 2477 & 2247 & 1864 \\ \text { BL1SPS3 } & 5846 & 3116 & 2921 & 2424 \\ \text { BL1SPS4 } & 6296 & 3357 & 3148 & 2612 \\ \text { BL1SPS5 } & 8516 & 4479 & 4250 & 3526 \\ \text { BL1SPS6 } & 10700 & 5561 & 5334 & 4426 \\ \text { BL1SPS7 } & 10640 & 5243 & 5607 & 4652 \\ \text { BL1SPS8 } & 17464 & 7769 & 8008 & 6645 \\ \text { BL1SPS9 } & 18989 & 9456 & 9425 & 7820 \\ \text { BL1SPS10 } & 18824 & 9387 & 9180 & 7617 \\ \text { BL1SPS11 } & 25007 & 12069 & 12376 & 10268 \\ \text { BL1SPS12 } & 22029 & 10795 & 10916 & 9057 \\ \text { BL1SPS13 } & 4334 & 2127 & 2148 & 1782 \\ \text { BL2SPS1 } & 3525 & 1713 & 1577 & 1315 \\ \text { BL2SPS2 } & 4782 & 2306 & 2082 & 1736 \\ \text { BL2SPS3 } & 6056 & 2901 & 2706 & 2257 \\ \text { BL2SPS4 } & 6522 & 3125 & 2916 & 2432 \\ \text { BL2SPS5 } & 8822 & 4170 & 3938 & 3283 \\ \text { BL2SPS6 } & 11085 & 5178 & 4942 & 4120 \\ \text { BL2SPS7 } & 11023 & 4882 & 5195 & 4331 \\ \text { BL2SPS8 } & 18092 & 7233 & 7420 & 6187 \\ \text { BL2SPS9 } & 19672 & 8804 & 8732 & 7281 \\ \text { BL2SPS10 } & 19501 & 8739 & 8505 & 7092 \\ \text { BL2SPS11 } & 25907 & 11236 & 11466 & 9560 \\ \text { BL2SPS12 } & 22821 & 10051 & 10113 & 8432 \\ \text { BL2SPS13 } & 4490 & 1980 & 1990 & 1659\end{array}$


Table 4: The four possible 'types' of reynolds numbers are displayed.

$\begin{array}{lrrrr}\text { Emperimant: } & \text { Gorlin } & \text { Analid } & \text { Gabbay } & \text { Gabbay } \\ & & & & \\ \text { TD1NPS1 } & 2458 & 1839 & 1746 & 1412 \\ \text { TD1NPS2 } & 3335 & 2477 & 2304 & 1864 \\ \text { TD1NPS3 } & 4223 & 3116 & 2996 & 2424 \\ \text { TD1NPS4 } & 4548 & 3357 & 3228 & 2612 \\ \text { TD1NPS5 } & 6152 & 4479 & 4359 & 3526 \\ \text { TD1NPS6 } & 7730 & 5561 & 5470 & 4426 \\ \text { TD1NPS7 } & 7687 & 5243 & 5750 & 4652 \\ \text { TD1NPS8 } & 12617 & 7769 & 8213 & 6645 \\ \text { TD1NPS9 } & 13719 & 9456 & 9666 & 7820 \\ \text { TD1NPS10 } & 13600 & 9387 & 9415 & 7617 \\ \text { TD1NPS11 } & 18067 & 12069 & 12692 & 10268 \\ \text { TD1NPS12 } & 15915 & 10795 & 11195 & 9057 \\ \text { TD1NPS13 } & 3131 & 2127 & 2203 & 1782 \\ & & & & \\ \text { TD2NPS1 } & 2120 & 1713 & 1589 & 1315 \\ \text { TD2NPS2 } & 2877 & 2306 & 2097 & 1736 \\ \text { TD2NPS3 } & 3644 & 2901 & 2726 & 2257 \\ \text { TD2NPS4 } & 3924 & 3125 & 2938 & 2432 \\ \text { TD2NPS5 } & 5308 & 4170 & 3967 & 3283 \\ \text { TD2NPS6 } & 6669 & 5178 & 4978 & 4120 \\ \text { TD2NPS7 } & 6632 & 4882 & 5233 & 4331 \\ \text { TD2NPS8 } & 10885 & 7233 & 7475 & 6187 \\ \text { TD2NPS9 } & 11835 & 8804 & 8797 & 7281 \\ \text { TD2NPS10 } & 11732 & 8739 & 8568 & 7092 \\ \text { TD2NPS11 } & 15586 & 11236 & 11551 & 9560 \\ \text { TD2NPS12 } & 13730 & 10051 & 10188 & 8432 \\ \text { TD2NPS13 } & 2701 & 1980 & 2004 & 1659 \\ \text { TD1MPS1 } & 3019 & 1839 & 1746 & 1412 \\ \text { TD1MPS2 } & 4096 & 2477 & 2304 & 1864 \\ \text { TD1MPS3 } & 5188 & 3116 & 2996 & 2424 \\ \text { TD1MPS4 } & 5587 & 3357 & 3228 & 2612 \\ \text { TD1MPS5 } & 7557 & 4479 & 4359 & 3526 \\ \text { TD1MPS6 } & 9495 & 5561 & 5470 & 4426 \\ \text { TD1MPS7 } & 9442 & 5243 & 5750 & 4652 \\ \text { TD1MPS8 } & 15497 & 7769 & 8213 & 6645 \\ \text { TD1MPS9 } & 16851 & 9456 & 9666 & 7820 \\ \text { TD1MPS10 } & 16704 & 9387 & 9415 & 7617 \\ \text { TD1MPS11 } & 22191 & 12069 & 12692 & 10268 \\ \text { TD1MPS12 } & 19548 & 10795 & 11195 & 9057 \\ \text { TD1MPS13 } & 3846 & 2127 & 2203 & 1782\end{array}$


Table 4: The four possible 'types' of reynolds numbers are displayed.

\begin{tabular}{|c|c|c|c|c|}
\hline Erperiment: & Gorlin & Aaslid & Gabbay & Gabbay \\
\hline TD2MPS1 & 2910 & 1713 & 1589 & 1315 \\
\hline TD2MPS2 & 3948 & 2306 & 2097 & 1736 \\
\hline TD2MPS 3 & 5000 & 2901 & 2726 & 2257 \\
\hline TD2MPS4 & 5384 & 3125 & 2938 & 2432 \\
\hline TD2MPS5 & 7283 & 4170 & 3967 & 3283 \\
\hline TD2MPS 6 & 9151 & 5178 & 4978 & 4120 \\
\hline TD2MPS7 & 9100 & 4882 & 5233 & 4331 \\
\hline TD2MPS8 & 14936 & 7233 & 7475 & 6187 \\
\hline TD2MPS9 & 16240 & 8804 & 8797 & 7281 \\
\hline TD2MPS10 & 16099 & 8739 & 8568 & 7092 \\
\hline TD2MPS11 & 21387 & 11236 & 11551 & 9560 \\
\hline TD2MPS12 & 18840 & 10051 & 10188 & 8432 \\
\hline TD2MPS13 & 3707 & 1980 & 2004 & 1659 \\
\hline TD1SPS1 & 4169 & 1839 & 1746 & 1412 \\
\hline TD1SPS2 & 5656 & 2477 & 2304 & 1864 \\
\hline TD1SPS 3 & 7163 & 3116 & 2996 & 2424 \\
\hline TD1SPS4 & 7714 & 3357 & 3228 & 2612 \\
\hline TD1SPS5 & 10435 & 4479 & 4359 & 3526 \\
\hline TD1SPS6 & 13111 & 5561 & 5470 & 4426 \\
\hline TD1SPS7 & 13038 & 5243 & 5750 & 4652 \\
\hline TD1SPS8 & 21399 & 7769 & 8213 & 6645 \\
\hline TD1SPS9 & 23268 & 9456 & 9666 & 7820 \\
\hline TD1SPS10 & 23066 & 9387 & 9415 & 7617 \\
\hline TD1SPS11 & 30643 & 12069 & 12692 & 10268 \\
\hline TD1SPS12 & 26993 & 10795 & 11195 & 9057 \\
\hline TD1SPS13 & 5311 & 2127 & 2203 & 1782 \\
\hline TD2SPS 1 & 3691 & 1713 & 1589 & 1315 \\
\hline TD2SPS2 & 5008 & 2306 & 2097 & 1736 \\
\hline TD2SPS 3 & 6342 & 2901 & 2726 & 2257 \\
\hline TD2SPS4 & 6830 & 3125 & 2938 & 2432 \\
\hline TD2SPS5 & 9239 & 4170 & 3967 & 3283 \\
\hline TD2SPS 6 & 11608 & 5178 & 4978 & 4120 \\
\hline TD2SPS7 & 11544 & 4882 & 5233 & 4331 \\
\hline TD2SPS 8 & 18947 & 7233 & 7475 & 6187 \\
\hline TD2SPS9 & 20601 & 8804 & 8797 & 7281 \\
\hline TD2SPS10 & 20422 & 8739 & 8568 & 7092 \\
\hline TD2SPS11 & 27131 & 11236 & 11551 & 9560 \\
\hline TD2SPS12 & 23899 & 10051 & 10188 & 8432 \\
\hline TD2SPS13 & 4702 & 1980 & 2004 & 1659 \\
\hline
\end{tabular}


Table 4: The four possible 'types' of reynolds numbers are displayed.

Experiment:
Corlin

$\operatorname{An}=1 i d$
Gabay

$\begin{array}{lrrrr}\text { BP1NPS1 } & 2931 & 1839 & 3011 & 1412 \\ \text { BP1NPS2 } & 3916 & 2477 & 3915 & 1864 \\ \text { BP1NPS3 } & 4641 & 3116 & 4762 & 2424 \\ \text { BP1NPS4 } & 5043 & 3357 & 5178 & 2612 \\ \text { BP1NPS5 } & 6786 & 4479 & 6954 & 3526 \\ \text { BP1NPS6 } & 7992 & 5561 & 8181 & 4426 \\ \text { BP1NPS7 } & 8088 & 5243 & 8751 & 4652 \\ \text { BP1NPS8 } & 12271 & 7769 & 11555 & 6645 \\ \text { BP1NPS9 } & 13638 & 9456 & 13900 & 7820 \\ \text { BP1NPS10 } & 13256 & 9387 & 13274 & 7617 \\ \text { BP1NPS11 } & 17400 & 12069 & 17682 & 10268 \\ \text { BP1NPS12 } & 15236 & 10795 & 15503 & 9057 \\ \text { BP1NPS13 } & 3533 & 2127 & 3595 & 1782 \\ & & & & \\ \text { BP2NPS1 } & 2553 & 1713 & 2623 & 1315 \\ \text { BP2NPS2 } & 3268 & 2306 & 3267 & 1736 \\ \text { BP2NPS3 } & 3878 & 2901 & 3979 & 2257 \\ \text { BP2NPS4 } & 4217 & 3125 & 4330 & 2432 \\ \text { BP2NPS5 } & 5568 & 4170 & 5707 & 3283 \\ \text { BP2NPS6 } & 6784 & 5178 & 6945 & 4120 \\ \text { BP2NPS7 } & 6259 & 4882 & 6773 & 4331 \\ \text { BP2NPS8 } & 10401 & 7233 & 9794 & 6187 \\ \text { BP2NPS9 } & 11520 & 8804 & 11741 & 7281 \\ \text { BP2NPS10 } & 11060 & 8739 & 11076 & 7092 \\ \text { BP2NPS11 } & 14622 & 11236 & 14859 & 9560 \\ \text { BP2NPS12 } & 12835 & 10051 & 13060 & 8432 \\ \text { BP2NPS13 } & 3042 & 1980 & 3095 & 1659 \\ \text { BP1MPS1 } & 2689 & 1839 & 2763 & 1412 \\ \text { BP1MPS2 } & 3432 & 2477 & 3431 & 1864 \\ \text { BP1MPS3 } & 4233 & 3116 & 4343 & 2424 \\ \text { BP1MPS4 } & 4773 & 3357 & 4900 & 2612 \\ \text { BP1MPS5 } & 6163 & 4479 & 6317 & 3526 \\ \text { BP1MPS6 } & 7660 & 5561 & 7841 & 4426 \\ \text { BP1MPS7 } & 7556 & 5243 & 8177 & 4652 \\ \text { BP1MPS8 } & 11688 & 7769 & 11006 & 6645 \\ \text { BP1MPS9 } & 12912 & 9456 & 13160 & 7820 \\ \text { BP1MPS10 } & 12406 & 9387 & 12423 & 7617 \\ \text { BP1MPS11 } & 16461 & 12069 & 16728 & 10268 \\ \text { BP1MPS12 } & 14666 & 10795 & 14923 & 9057 \\ \text { BP1MPS13 } & 3769 & 2127 & 3835 & 1782\end{array}$


Table 4: The four possible 'types' of reynolds numbers are displayed.

Inperiment:
Corlin

Anslid
Gabbay

$\begin{array}{rrr}1713 & 4041 & 1315 \\ 2306 & 4396 & 1736 \\ 2901 & 5321 & 2257 \\ 3125 & 5334 & 2432 \\ 4170 & 6596 & 3283 \\ 5178 & 7722 & 4120 \\ 4882 & 7979 & 4331 \\ 7233 & 10742 & 6187 \\ 8804 & 12492 & 7281 \\ 8739 & 12204 & 7092 \\ 11236 & 15619 & 9560 \\ 10051 & 13983 & 8432 \\ 1980 & 3677 & 1659 \\ & & \\ 1839 & 3482 & 1412 \\ 2477 & 4336 & 1864 \\ 3116 & 5303 & 2424 \\ 3357 & 5433 & 2612 \\ 4479 & 7234 & 3526 \\ 5561 & 8608 & 4426 \\ 5243 & 8863 & 4652 \\ 7769 & 12314 & 6645 \\ 9456 & 13371 & 7820 \\ 9387 & 13587 & 7617 \\ 12069 & 16885 & 10268 \\ 10795 & 14790 & 9057 \\ 2127 & 3845 & 1782 \\ 1713 & 4773 & 1315 \\ 2306 & 5934 & 1736 \\ 2901 & 7385 & 2257 \\ 3125 & 7001 & 2432 \\ 4170 & 9307 & 3283 \\ 5178 & 10885 & 4120 \\ 4882 & 11125 & 4331 \\ 7233 & 14994 & 6187 \\ 8804 & 16658 & 7281 \\ 8739 & 16497 & 7092 \\ 11236 & 20930 & 9560 \\ 10051 & 17976 & 8432 \\ 1980 & 4572 & 1659\end{array}$


Table 5: The four performance parameters obtained from the experiments. The left-most column is the experiment designation, followed by the Gorlin coefficient of discharge, the Aaslid effective area index, the Gabbay coefficient of discharge and the Gabbay performance index.

\begin{tabular}{|c|c|c|c|c|c|}
\hline Experiment & Gorlin & Anslid & Gabbay & cd & Gabb \\
\hline BL1NPS 1 & 0.900 & 0.637 & 0.763 & & 0.52 \\
\hline BL1NPS2 & 0.911 & 0.611 & 0.752 & & 0.51 \\
\hline BL1NPS 3 & 1.065 & 0.858 & 0.902 & & 0.62 \\
\hline BL1NPS4 & 1.102 & 0.672 & 0.934 & & 0.64 \\
\hline BLINPS5 & 1.093 & 0.557 & 0.924 & & 0.63 \\
\hline BL1NPS 6 & 1.163 & 0.728 & 0.982 & & 0.67 \\
\hline BL1NPS7 & 0.817 & 0.645 & 0.730 & & 0.50 \\
\hline BL1NPS 8 & 1.108 & 0.678 & 0.861 & & 0.59 \\
\hline BL1NPS9 & 0.986 & 0.664 & 0.830 & & 0.57 \\
\hline BL1NPS10 & 0.928 & 0.575 & 0.767 & & 0.52 \\
\hline BL1NPS1 1 & 1.027 & 0.660 & 0.862 & & 0.59 \\
\hline BL 1 NPS 12 & 0.999 & 0.623 & 0.839 & & 0.57 \\
\hline BL 1 NPS 13 & 1.467 & 0.213 & 1.232 & & 0.84 \\
\hline BL2NPS 1 & 0.636 & 0.302 & 0.527 & & 0.36 \\
\hline BL2NPS2 & 0.716 & 0.622 & 0.577 & & 0.40 \\
\hline BL2NPS 3 & 0.749 & 0.521 & 0.620 & & 0.43 \\
\hline BL2NPS4 & 0.767 & 0.502 & 0.635 & & 0.44 \\
\hline BL2NPS5 & 0.977 & 0.537 & 0.808 & & 0.56 \\
\hline BL2NPS6 & 0.918 & 0.592 & 0.758 & & 0.52 \\
\hline BL2NPS 7 & 0.837 & 0.535 & 0.731 & & $0.5 \mathrm{C}$ \\
\hline BL2NPS8 & 0.675 & 0.322 & 0.513 & & 0.35 \\
\hline BL 2NPS 9 & 0.991 & 0.695 & 0.815 & & 0.56 \\
\hline BL2NPS10 & 0.914 & 0.748 & 0.739 & & 0.51 \\
\hline BL2NPS 11 & 1.022 & 0.667 & 0.838 & & $0.5 \varepsilon$ \\
\hline BL 2NPS 12 & 1.003 & 0.505 & 0.823 & & 0.57 \\
\hline BL2NPS 13 & 1.463 & 0.212 & 1.201 & & 0.83 \\
\hline BL 1MPS1 & 0.605 & 0.225 & 0.303 & & 0.21 \\
\hline BL1MPS2 & 0.650 & 0.279 & 0.317 & & 0.21 \\
\hline BL1MPS 3 & 0.778 & 0.308 & 0.390 & & 0.26 \\
\hline BL1MPS4 & 0.771 & 0.337 & 0.386 & & 0.26 \\
\hline BL 1MPS5 & 0.875 & 0.381 & 0.438 & & 0.31 \\
\hline BL1MPS 6 & 0.958 & 0.397 & 0.479 & & $0.3:$ \\
\hline BL1MPS7 & 0.967 & 0.414 & 0.511 & & 0.35 \\
\hline BL1MPS 8 & 1.252 & 0.467 & 0.576 & & 0.35 \\
\hline BL1MPS 9 & 1.128 & 0.455 & 0.561 & & 0.38 \\
\hline BL $1 M P S 10$ & 1.136 & 0.447 & 0.555 & & 0.38 \\
\hline BL 1MPS 11 & 1.212 & 0.420 & 0.601 & & 0.43 \\
\hline BL 1 MPS 12 & 1.016 & 0.372 & 0.505 & & 0.34 \\
\hline BL 1MPS 13 & 0.852 & 0.224 & 0.423 & & 0.25 \\
\hline
\end{tabular}


Table 5: The four performance parameters obtained from the experiments. The left-most column is the experiment designation, followed by the Gorlin coefficient of discharge, the Aaslid effective area index, the Gabbay coefficient of discharge and the Gabbay performance index.

\begin{tabular}{|c|c|c|c|c|}
\hline Exporiment & Corlin & Anslid & Gabbay Cd & Gabb \\
\hline BL2MPS1 & 0.594 & 0.189 & 0.236 & 0.16 \\
\hline BL2MPS2 & 0.733 & 0.247 & 0.283 & 0.19 \\
\hline BL2MPS3 & 0.823 & 0.260 & 0.326 & 0.22 \\
\hline BL2MPS4 & 0.897 & 0.275 & 0.356 & 0.24 \\
\hline BL 2MPS5 & 0.904 & 0.298 & 0.358 & 0.24 \\
\hline BL2MPS6 & 0.968 & 0.321 & 0.383 & \\
\hline BL 2MPS 7 & 0.924 & 0.308 & 0.386 & 0.26 \\
\hline BL 2MPS8 & 1.183 & 0.344 & 0.430 & 0.25 \\
\hline BL2MPS9 & 1.068 & 0.347 & 0.421 & 0.25 \\
\hline BL 2MPS10 & 1.069 & 0.317 & 0.414 & \\
\hline BL2MPS 11 & 1.173 & 0.336 & 0.461 & 0.37 \\
\hline BL 2MPS 12 & 1.138 & 0.316 & 0.447 & 0.31 \\
\hline BL 2MPS1 3 & 1.221 & 0.211 & 0.480 & \\
\hline BL1SPS1 & 1.077 & 0.221 & 0.262 & 0.18 \\
\hline BL1SPS2 & 1.052 & 0.194 & 0.249 & 0.1 \\
\hline BL1SPS3 & 1.027 & 0.188 & 0.250 & $0.1 \%$ \\
\hline BL1SPS4 & 1.059 & 0.212 & 0.258 & 0.17 \\
\hline BL1SPS5 & 1.066 & 0.206 & 0.259 & 0.17 \\
\hline BL1SPS6 & 1.043 & 0.185 & 0.253 & 0.17 \\
\hline BL1SPS7 & 1.040 & 0.186 & 0.267 & 0.18 \\
\hline BL1SPS8 & 1.327 & 0.221 & 0.296 & 0.21 \\
\hline BL1SPS9 & 1.177 & 0.226 & 0.284 & 0.15 \\
\hline BL1SPS10 & 1.230 & 0.247 & 0.292 & 0.21 \\
\hline BL1SPS11 & 1.317 & 0.252 & 0.317 & 0.21 \\
\hline BL1SPS 12 & 1.241 & 0.238 & 0.299 & 0.21 \\
\hline BL1SPS13 & 1.255 & 0.249 & 0.303 & 0.20 \\
\hline BL2SPS1 & 1.046 & 0.163 & 0.204 & 0.14 \\
\hline BL2SPS2 & 0.992 & 0.146 & 0.188 & $0.1:$ \\
\hline BL2SPS 3 & 0.996 & 0.148 & 0.194 & $0.1:$ \\
\hline BL2SPS4 & 1.040 & 0.158 & 0.203 & 0.14 \\
\hline BL2SPS5 & 1.026 & 0.154 & 0.199 & $0.1:$ \\
\hline BL2SPS 6 & 1.038 & 0.159 & 0.202 & 0.14 \\
\hline BL2SPS 7 & 1.015 & 0.152 & 0.208 & 0.14 \\
\hline BL2SPS 8 & 1.297 & 0.172 & 0.232 & 0.16 \\
\hline BL2SPS9 & 1.160 & 0.169 & 0.224 & 0.15 \\
\hline BL2SPS10 & 1.237 & 0.180 & 0.235 & 0.16 \\
\hline BL2SPS11 & 1.357 & 0.188 & 0.261 & 0.18 \\
\hline BL2SPS 12 & 1.222 & 0.178 & 0.236 & 0.16 \\
\hline BL2SPS13 & 1.199 & 0.186 & 0.231 & 0.1 \\
\hline
\end{tabular}


Table 5: The four performance parameters obtained from the experiments. The left-most column is the experiment designation, followed by the Gorlin coefficient of discharge, the Aaslid effective area index, the Gabbay coefficient of discharge and the Gabbay performance index.

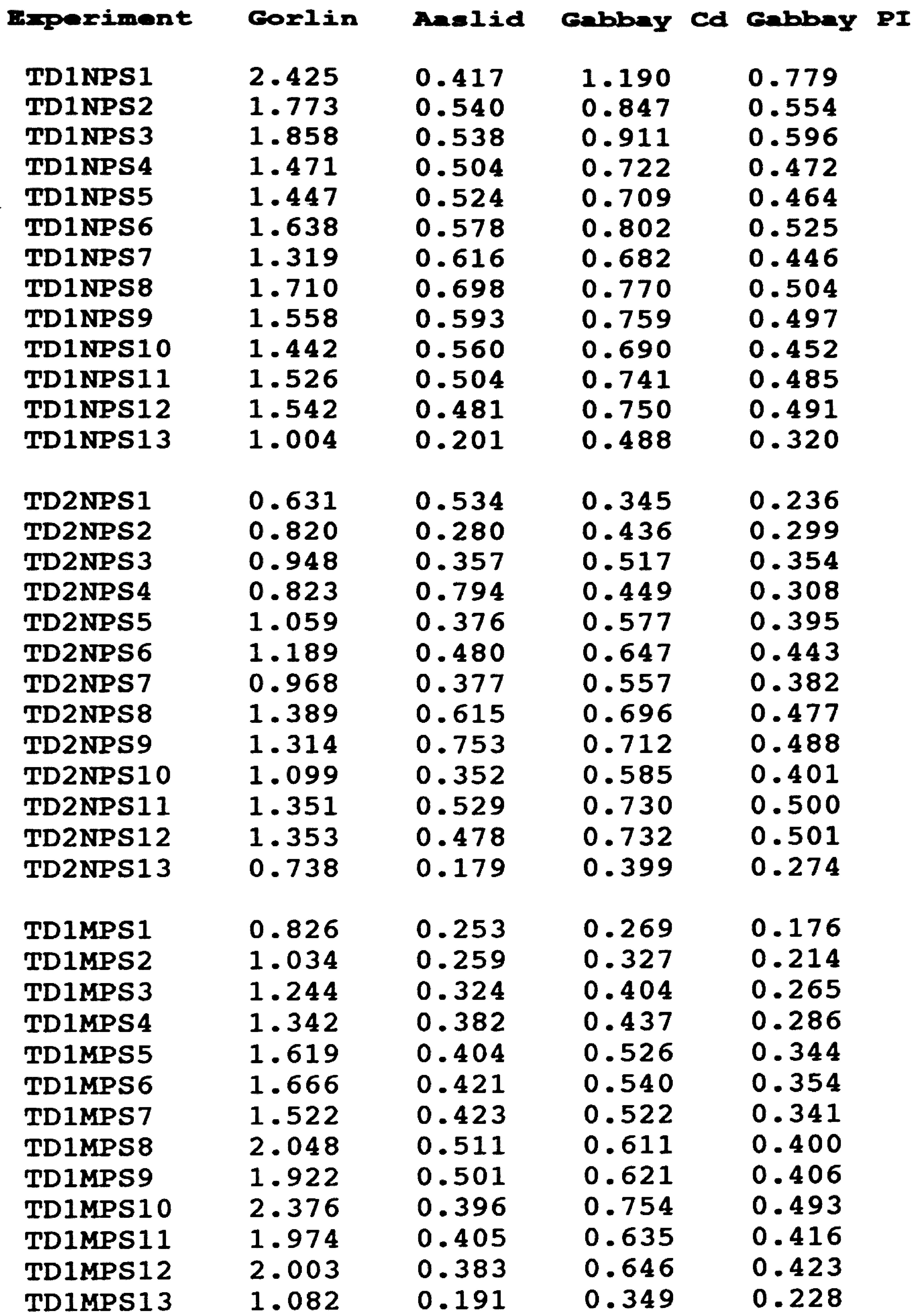


Table 5: The four performance parameters obtained from the experiments. The left-most column is the experiment designation, followed by the Gorlin coefficient of discharge, the Aaslid effective area index, the Gabbay coefficient of discharge and the Gabbay performance index.

$\begin{array}{lllll}\text { EDPeriment } & \text { Gorlin } & \text { Aaslid } & \text { Gabbay Cd GabbaY PI } \\ \text { TD2MPS1 } & 1.895 & 0.478 & 0.550 & 0.377 \\ \text { TD2MPS2 } & 1.872 & 0.369 & 0.529 & 0.362 \\ \text { TD2MPS3 } & 1.892 & 0.412 & 0.548 & 0.376 \\ \text { TD2MPS4 } & 1.784 & 0.603 & 0.517 & 0.354 \\ \text { TD2MPS5 } & 1.872 & 0.459 & 0.542 & 0.371 \\ \text { TD2MPS6 } & 1.958 & 0.470 & 0.566 & 0.388 \\ \text { TD2MPS7 } & 1.686 & 0.469 & 0.515 & 0.353 \\ \text { TD2MPS8 } & 2.212 & 0.496 & 0.588 & 0.403 \\ \text { TD2MPS9 } & 1.998 & 0.475 & 0.575 & 0.394 \\ \text { TD2MPS10 } & 1.969 & 0.402 & 0.557 & 0.381 \\ \text { TD2MPS11 } & 2.059 & 0.426 & 0.591 & 0.405 \\ \text { TD2MPS12 } & 1.990 & 0.387 & 0.572 & 0.392 \\ \text { TD2MPS13 } & 1.427 & 0.193 & 0.410 & 0.281 \\ \text { TD1SPS1 } & 3.574 & 0.513 & 0.610 & 0.399 \\ \text { TD1SPS2 } & 3.059 & 0.443 & 0.508 & 0.332 \\ \text { TD1SPS3 } & 2.856 & 0.361 & 0.487 & 0.319 \\ \text { TD1SPS4 } & 2.841 & 0.405 & 0.485 & 0.317 \\ \text { TD1SPS5 } & 2.804 & 0.395 & 0.477 & 0.313 \\ \text { TD1SPS6 } & 2.782 & 0.360 & 0.473 & 0.310 \\ \text { TD1SPS7 } & 2.585 & 0.349 & 0.465 & 0.304 \\ \text { TD1SPS8 } & 3.284 & 0.356 & 0.514 & 0.336 \\ \text { TD1SPS9 } & 2.883 & 0.331 & 0.488 & 0.320 \\ \text { TD1SPS10 } & 2.786 & 0.335 & 0.463 & 0.303 \\ \text { TD1SPS11 } & 3.024 & 0.343 & 0.510 & 0.334 \\ \text { TD1SPS12 } & 2.976 & 0.328 & 0.503 & 0.329 \\ \text { TD1SPS13 } & 2.564 & 0.209 & 0.433 & 0.284 \\ \text { TD2SPS1 } & 2.413 & 0.455 & 0.435 & 0.298 \\ \text { TD2SPS2 } & 2.169 & 0.299 & 0.380 & 0.261 \\ \text { TD2SPS3 } & 1.479 & 0.208 & 0.266 & 0.182 \\ \text { TD2SPS4 } & 1.548 & 0.236 & 0.279 & 0.191 \\ \text { TD2SPS5 } & 1.752 & 0.270 & 0.315 & 0.216 \\ \text { TD2SPS6 } & 1.828 & 0.260 & 0.328 & 0.225 \\ \text { TD2SPS7 } & 1.802 & 0.246 & 0.342 & 0.234 \\ \text { TD2SPS8 } & 2.363 & 0.300 & 0.391 & 0.268 \\ \text { TD2SPS9 } & 2.082 & 0.282 & 0.373 & 0.255 \\ \text { TD2SPS10 } & 2.126 & 0.284 & 0.374 & 0.256 \\ \text { TD2SPS11 } & 2.198 & 0.287 & 0.392 & 0.269 \\ \text { TD2SPS12 } & 2.273 & 0.283 & 0.406 & 0.278 \\ \text { TD2SPS13 } & 2.100 & 0.208 & 0.375 & 0.257\end{array}$


Table 5: The four performance parameters obtained from the experiments. The left-most column is the experiment designation, followed by the Gorlin coefficient of discharge, the Aaslid effective area index, the Gabbay coefficient of discharge and the Gabbay performance index.

\begin{tabular}{|c|c|c|c|c|}
\hline srperiment & Corlin & Anslid & Gabbay Cd & Gabbas \\
\hline $\begin{array}{l}\text { BP1NPS1 } \\
\text { BP1NPS } 2 \\
\text { BP1NPS } 3 \\
\text { BP1NPS4 } \\
\text { BP1NPS5 } \\
\text { BP1NPS } 6 \\
\text { BP1NPS7 } \\
\text { BP1NPS8 } \\
\text { BP1NPS9 } \\
\text { BP1NPS10 } \\
\text { BP1NPS11 } \\
\text { BP1NPS12 } \\
\text { BP1NPS13 }\end{array}$ & $\begin{array}{l}1.072 \\
1.094 \\
1.036 \\
1.057 \\
1.131 \\
1.065 \\
1.035 \\
1.174 \\
1.116 \\
1.037 \\
1.106 \\
1.074 \\
0.909\end{array}$ & $\begin{array}{l}0.577 \\
0.332 \\
0.372 \\
0.295 \\
0.308 \\
0.324 \\
0.267 \\
0.325 \\
0.334 \\
0.350 \\
0.352 \\
0.358 \\
0.187\end{array}$ & $\begin{array}{l}1.101 \\
1.094 \\
1.063 \\
1.085 \\
1.159 \\
1.090 \\
1.120 \\
1.105 \\
1.137 \\
1.039 \\
1.123 \\
1.093 \\
0.925\end{array}$ & $\begin{array}{l}0.242 \\
0.248 \\
0.275 \\
0.276 \\
0.298 \\
0.319 \\
0.316 \\
0.366 \\
0.360 \\
0.342 \\
0.379 \\
0.373 \\
0.227\end{array}$ \\
\hline $\begin{array}{l}\text { BP2NPS1 } \\
\text { BP2NPS2 } \\
\text { BP2NPS } 3 \\
\text { BP2NPS4 } \\
\text { BP2NPS5 } \\
\text { BP2NPS6 } \\
\text { BP2NPS } 7 \\
\text { BP2NPS8 } \\
\text { BP2NPS9 } \\
\text { BP2NPS } 10 \\
\text { BP2NPS } 11 \\
\text { BP2NPS } 12 \\
\text { BP2NPS } 13\end{array}$ & $\begin{array}{l}0.767 \\
0.820 \\
0.800 \\
0.800 \\
0.837 \\
0.912 \\
0.709 \\
0.949 \\
0.898 \\
0.829 \\
0.875 \\
0.878 \\
0.682\end{array}$ & $\begin{array}{l}0.290 \\
0.427 \\
0.389 \\
0.294 \\
0.305 \\
0.335 \\
0.267 \\
0.322 \\
0.321 \\
0.348 \\
0.341 \\
0.357 \\
0.179\end{array}$ & $\begin{array}{l}0.788 \\
0.819 \\
0.821 \\
0.821 \\
0.858 \\
0.934 \\
0.768 \\
0.894 \\
0.916 \\
0.830 \\
0.889 \\
0.894 \\
0.694\end{array}$ & $\begin{array}{l}0.198 \\
0.231 \\
0.264 \\
0.259 \\
0.284 \\
0.329 \\
0.314 \\
0.357 \\
0.352 \\
0.340 \\
0.368 \\
0.373 \\
0.200\end{array}$ \\
\hline $\begin{array}{l}\text { BP1MPS1 } \\
\text { BP1MPS } 2 \\
\text { BP1MPS } 3 \\
\text { BP1MPS4 } \\
\text { BP1MPS5 } \\
\text { BP1MPS } 6 \\
\text { BP1MPS } 7 \\
\text { BP1MPS } 8 \\
\text { BP1MPS9 } \\
\text { BP1MPS } 10 \\
\text { BP1MPS } 11 \\
\text { BP 1MPS } 12 \\
\text { BP 1MPS } 13\end{array}$ & $\begin{array}{l}0.964 \\
0.933 \\
0.875 \\
0.944 \\
0.924 \\
0.940 \\
0.875 \\
1.020 \\
0.949 \\
0.875 \\
0.922 \\
0.925 \\
1.021\end{array}$ & $\begin{array}{l}0.356 \\
0.429 \\
0.370 \\
0.309 \\
0.283 \\
0.308 \\
0.251 \\
0.298 \\
0.313 \\
0.329 \\
0.328 \\
0.326 \\
0.238\end{array}$ & $\begin{array}{l}0.990 \\
0.932 \\
0.898 \\
0.969 \\
0.947 \\
0.962 \\
0.947 \\
0.961 \\
0.968 \\
0.877 \\
0.937 \\
0.942 \\
1.039\end{array}$ & $\begin{array}{l}0.259 \\
0.275 \\
0.280 \\
0.275 \\
0.295 \\
0.307 \\
0.307 \\
0.350 \\
0.342 \\
0.330 \\
0.353 \\
0.347 \\
0.224\end{array}$ \\
\hline
\end{tabular}


Table 5: The four performance parameters obtained from the experiments. The left-most column is the experiment designation, followed by the Gorlin coefficient of discharge, the Aaslid effective area index, the Gabbay coefficient of discharge and the Gabbay performance index.

$\begin{array}{lllll}\text { ErPeriment } & \text { Gorlin } & \text { Aas1id } & \text { Gabbay } & \text { Cd GabbaY PI } \\ \text { BP2MPS1 } & 1.290 & 0.169 & 1.325 & 0.140 \\ \text { BP2MPS2 } & 0.958 & 0.182 & 0.958 & 0.149 \\ \text { BP2MPS3 } & 0.929 & 0.198 & 0.953 & 0.171 \\ \text { BP2MPS4 } & 0.920 & 0.198 & 0.945 & 0.196 \\ \text { BP2MPS5 } & 0.865 & 0.218 & 0.886 & 0.220 \\ \text { BP2MPS6 } & 0.858 & 0.259 & 0.879 & 0.250 \\ \text { BP2MPS7 } & 0.817 & 0.219 & 0.884 & 0.260 \\ \text { BP2MPS8 } & 0.971 & 0.268 & 0.915 & 0.303 \\ \text { BP2MPS9 } & 0.885 & 0.287 & 0.902 & 0.306 \\ \text { BP2MPS10 } & 0.930 & 0.303 & 0.931 & 0.314 \\ \text { BP2MPS11 } & 0.905 & 0.323 & 0.920 & 0.345 \\ \text { BP2MPS12 } & 0.960 & 0.331 & 0.977 & 0.355 \\ \text { BP2MPS13 } & 0.978 & 0.186 & 0.995 & 0.203 \\ \text { BP1SPS1 } & 0.958 & 0.195 & 0.984 & 0.162 \\ \text { BP1SPS2 } & 0.846 & 0.188 & 0.845 & 0.156 \\ \text { BP1SPS3 } & 0.908 & 0.225 & 0.932 & 0.195 \\ \text { BP1SPS4 } & 0.906 & 0.213 & 0.930 & 0.215 \\ \text { BP1SPS5 } & 0.958 & 0.227 & 0.982 & 0.233 \\ \text { BP1SPS6 } & 0.909 & 0.239 & 0.931 & 0.246 \\ \text { BP1SPS7 } & 0.879 & 0.223 & 0.951 & 0.262 \\ \text { BP1SPS8 } & 1.121 & 0.273 & 1.055 & 0.307 \\ \text { BP1SPS9 } & 0.924 & 0.301 & 0.942 & 0.322 \\ \text { BP1SPS10 } & 1.017 & 0.329 & 1.018 & 0.320 \\ \text { BP1SPS11 } & 0.926 & 0.331 & 0.941 & 0.348 \\ \text { BP1SPS12 } & 0.918 & 0.333 & 0.934 & 0.350 \\ \text { BP1SPS13 } & 1.143 & 0.226 & 1.163 & 0.250 \\ \text { BP2SPS1 } & 0.970 & 0.096 & 0.996 & 0.076 \\ \text { BP2SPS2 } & 0.942 & 0.092 & 0.942 & 0.081 \\ \text { BP2SPS3 } & 0.975 & 0.103 & 1.001 & 0.093 \\ \text { BP2SPS4 } & 0.977 & 0.131 & 1.004 & 0.121 \\ \text { BP2SPS5 } & 0.996 & 0.136 & 1.020 & 0.127 \\ \text { BP2SPS6 } & 0.945 & 0.150 & 0.967 & 0.139 \\ \text { BP2SPS7 } & 0.945 & 0.146 & 1.022 & 0.155 \\ \text { BP2SPS8 } & 1.136 & 0.183 & 1.070 & 0.182 \\ \text { BP2SPS9 } & 0.983 & 0.200 & 1.002 & 0.192 \\ \text { BP2SPS10 } & 1.144 & 0.224 & 1.146 & 0.212 \\ \text { BP2SPS11 } & 1.096 & 0.231 & 1.114 & 0.232 \\ \text { BP2SPS12 } & 0.989 & 0.226 & 1.006 & 0.221 \\ \text { BP2SPS13 } & 1.240 & 0.191 & 1.262 & 0.166\end{array}$



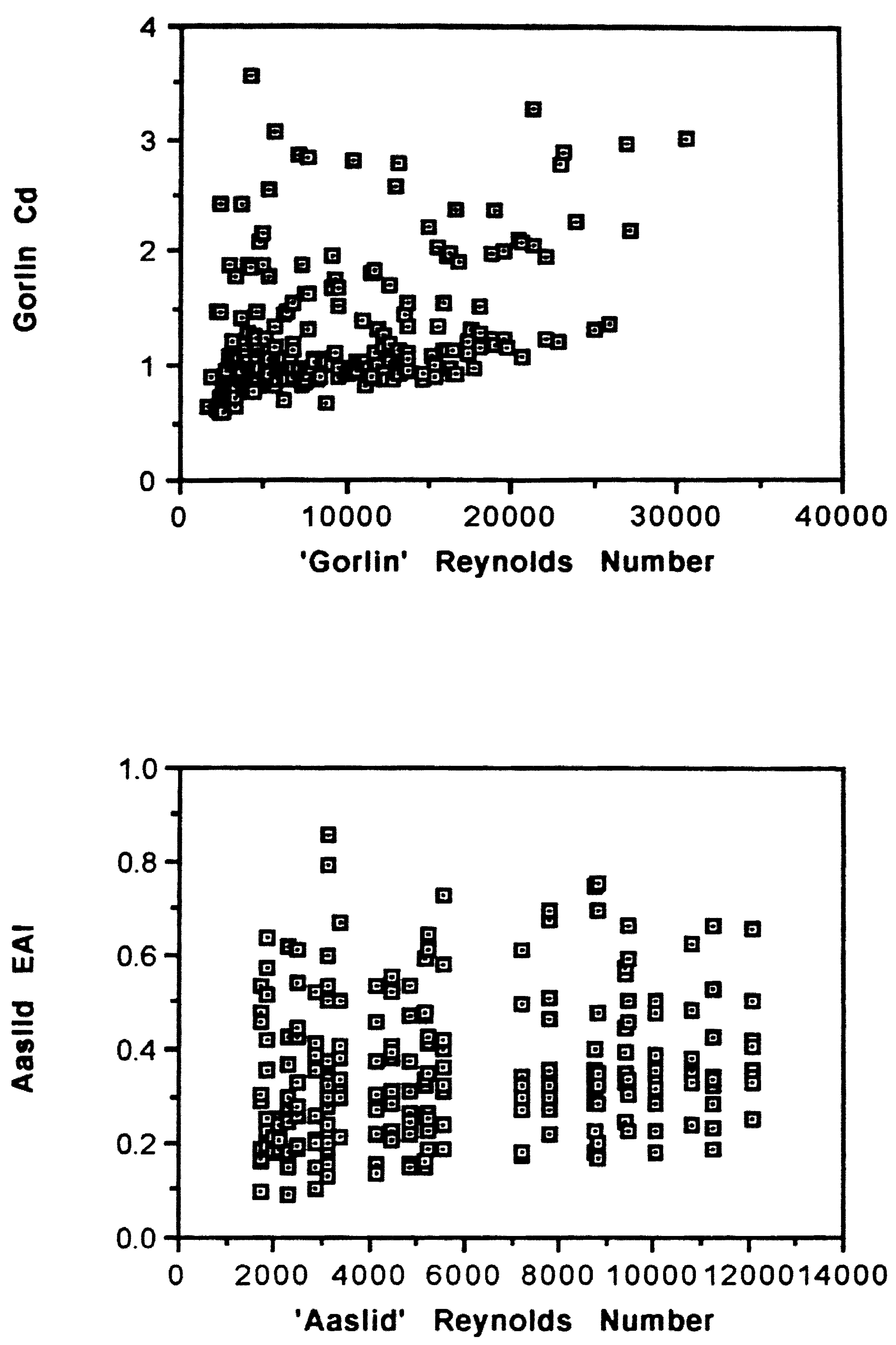

Figure 36: Population of 234 data points. Performance parameter is plotted as a function of Reynolds number. Note such huge variation in the values plotted. 

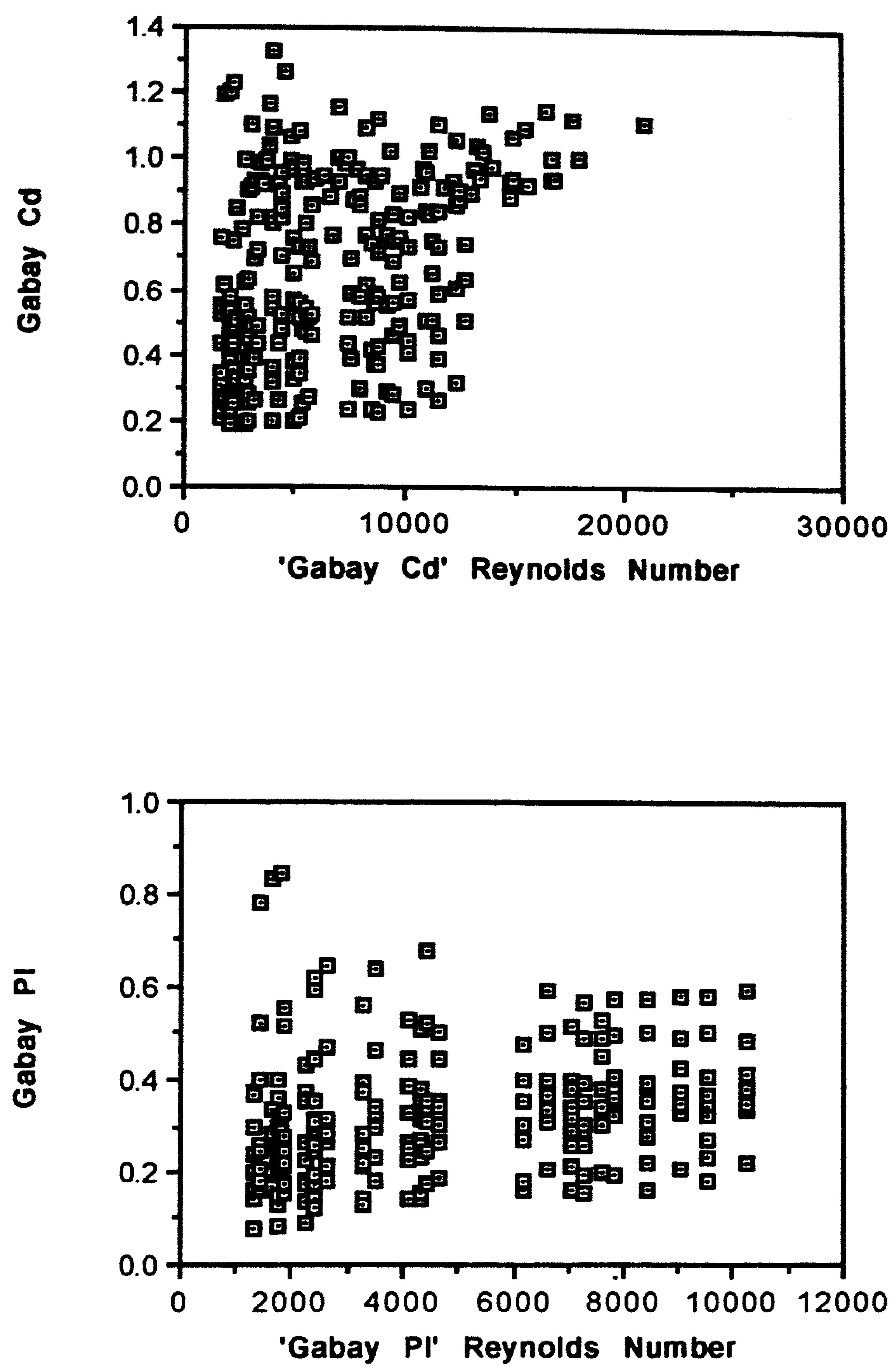

Figure 37: Population of 234 data points. Performance parameter is plotted as a function of Reynolds number. Note such huge variation in the values plotted. 

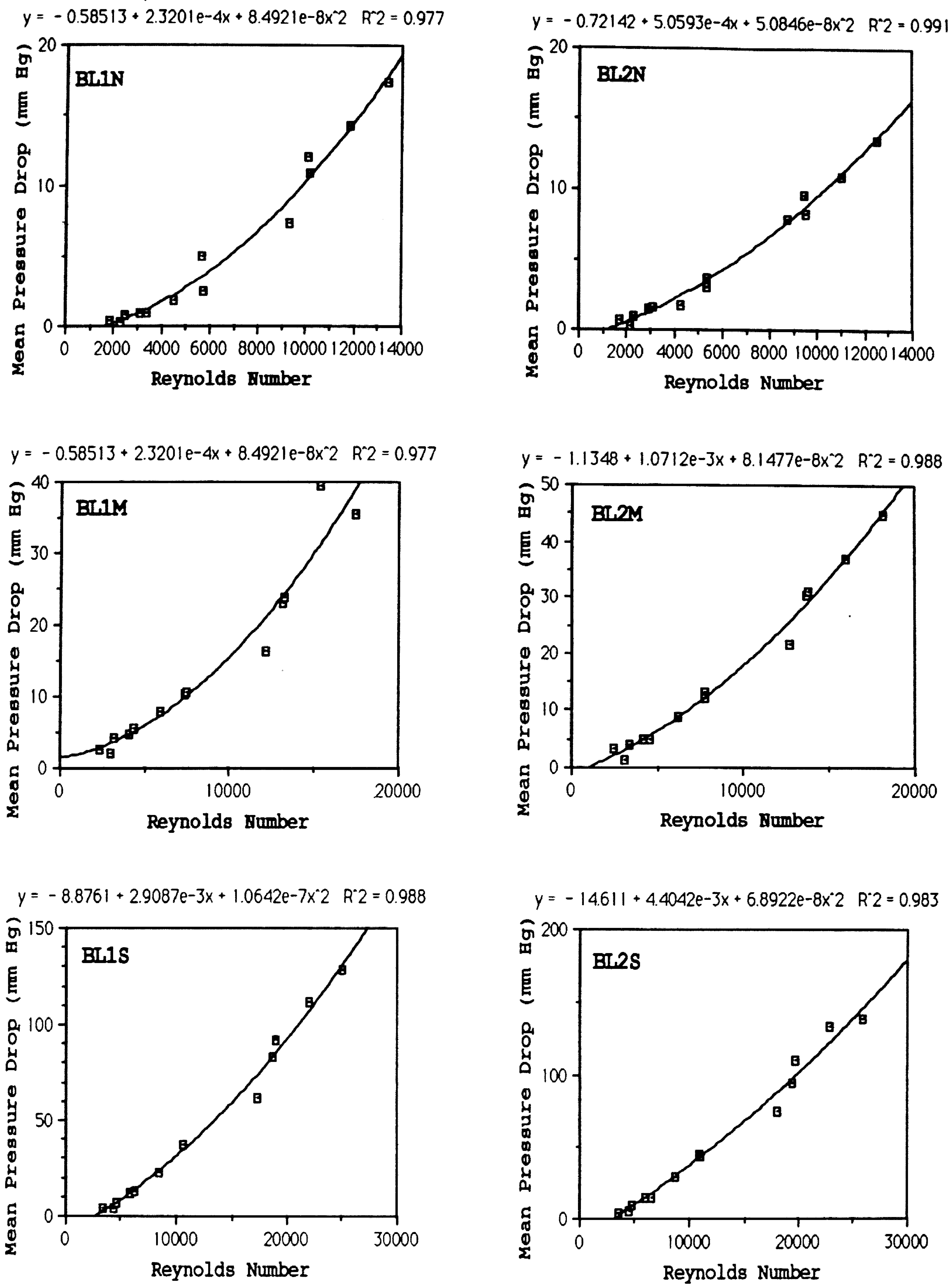

Figure 38: Pressure drop is plotted against Reynolds Number for all experiments with Bileaflet valve. Note second order regression line. 

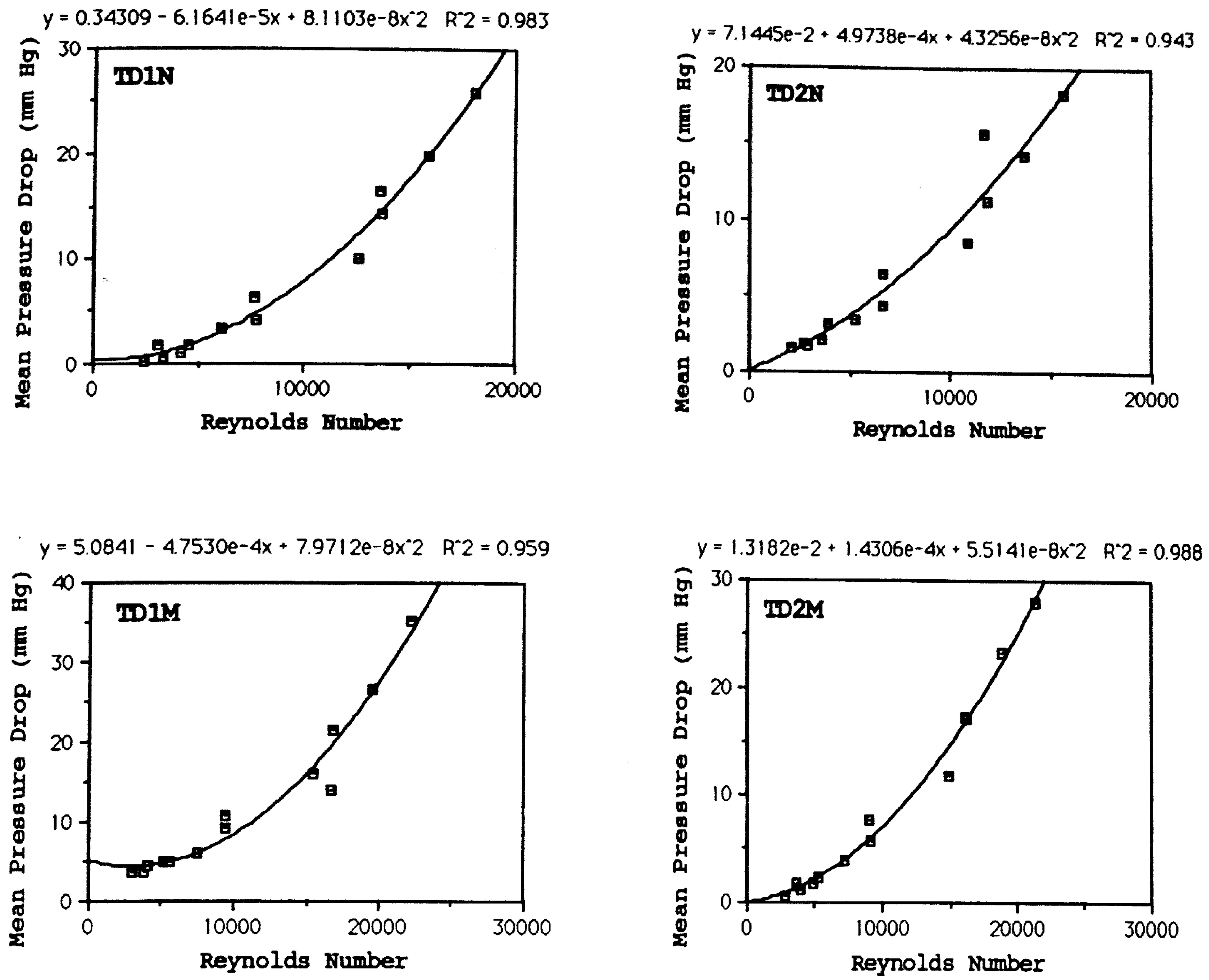

$y=-1.7336+4.1381 e-4 x+4.6979 e-8 x^{*} 2 R^{*} 2=0.986$
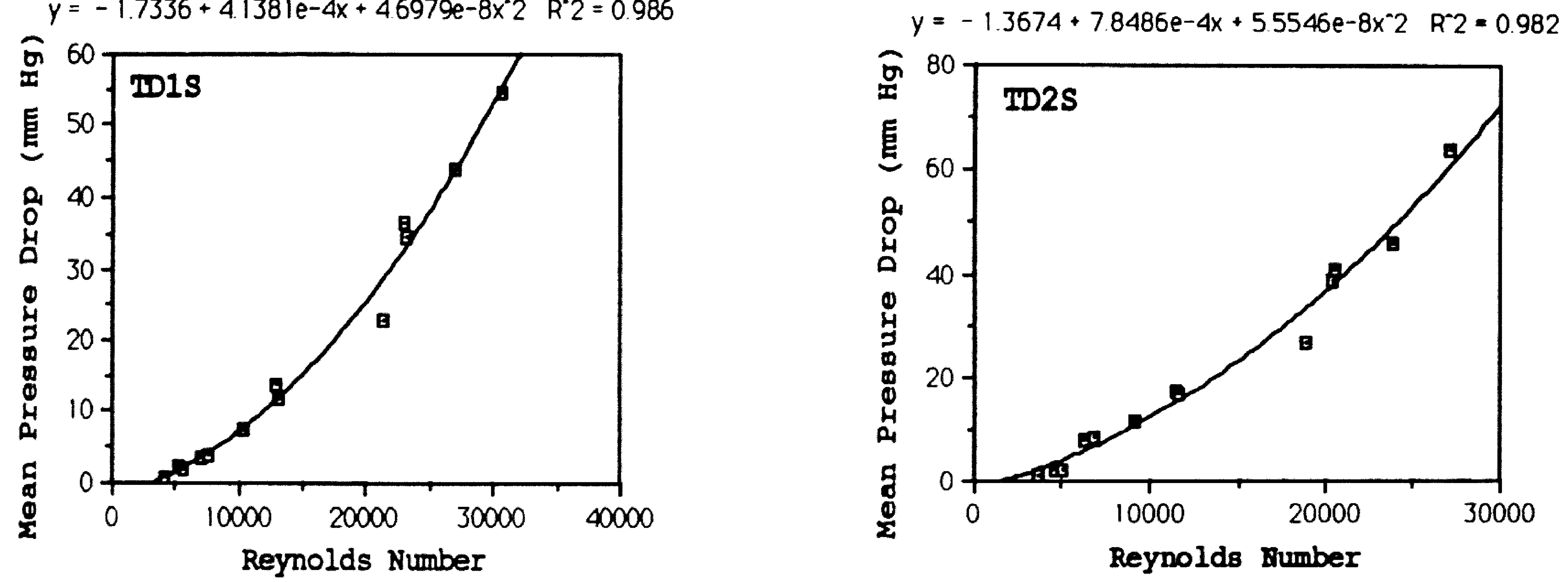

Figure 39: Pressure drop is plotted as a function of Reynolds Number for experiments with Tilting Disc valve. Note second order regression line. 

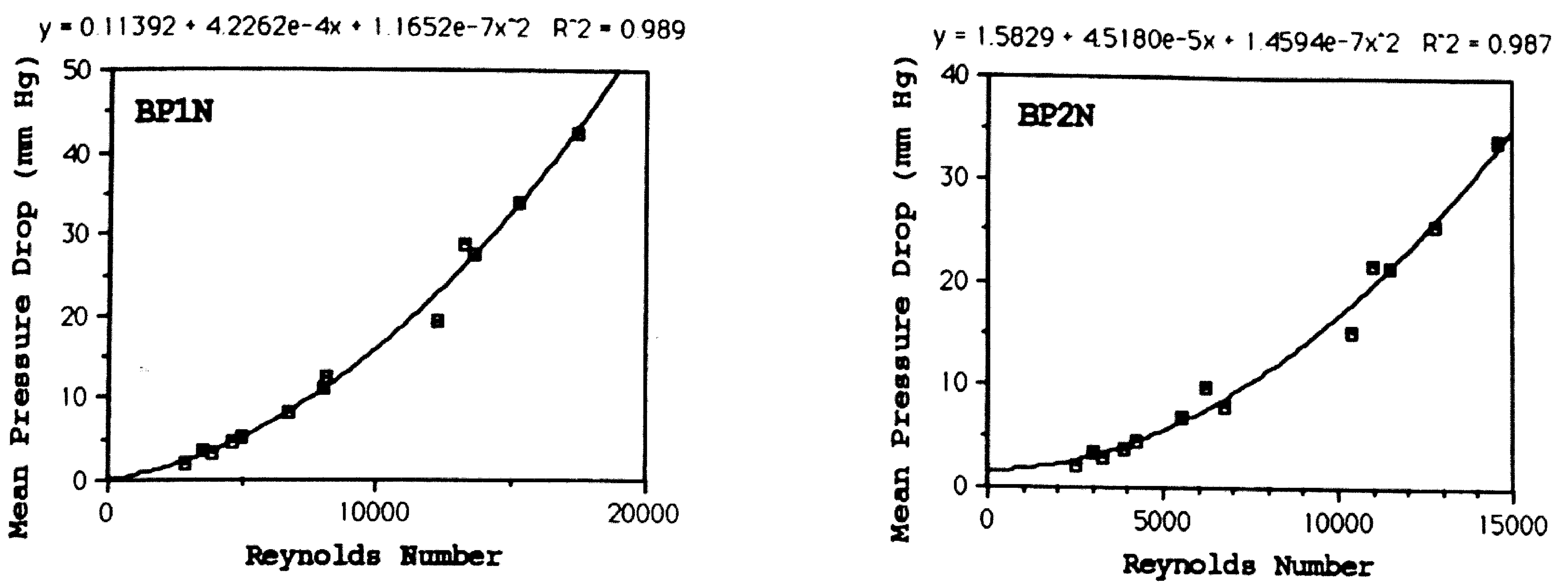

$y=0.42262+2.1262 e-4 x+1.6514 e-7 x^{\circ} 2 \quad R^{2} 2=0.989$
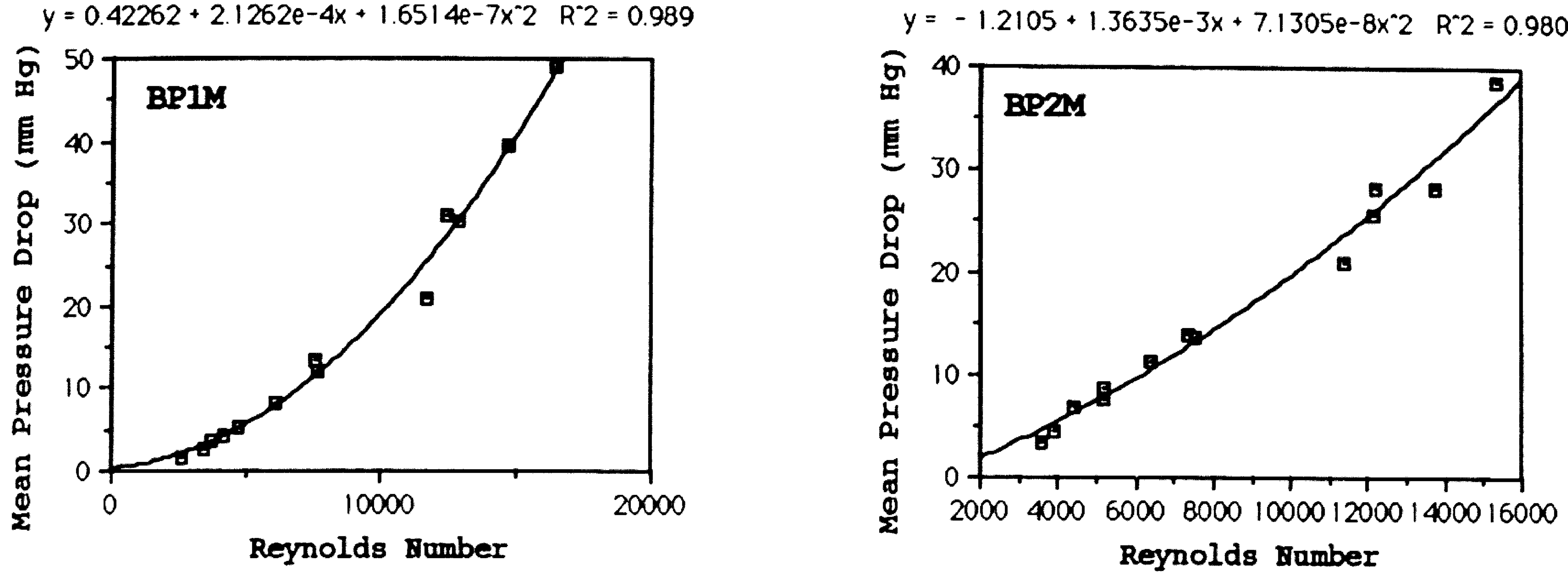

$y=7.0800 e-2+1.0548 e-3 x+1.0962 e-7 x^{*} 2 R 2=0977$
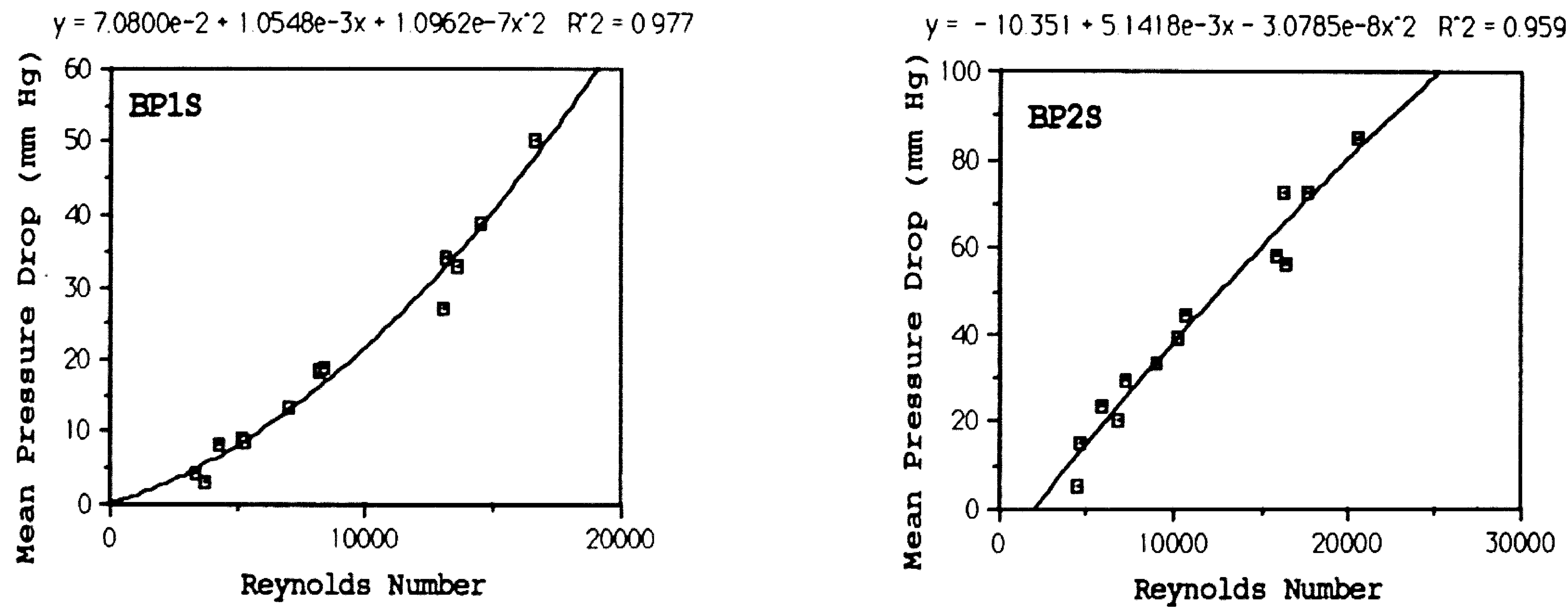

Figure 40: Pressure drop is plotted as a function of Reynolds Number for experiments with Bio Prosthetic valve. Note second order regression line. 

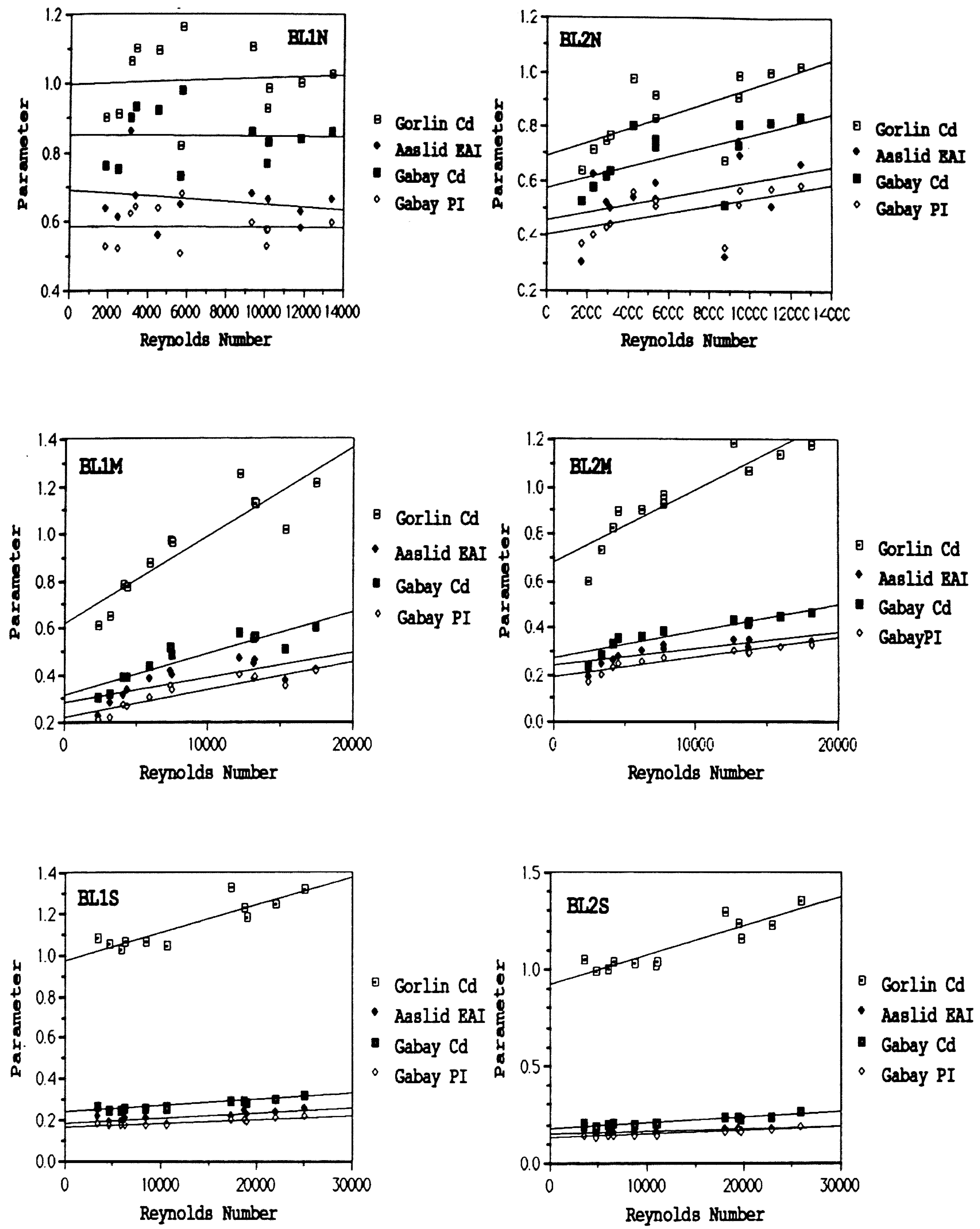

Figure 41: Four performance parameters are plotted as a function of Reynolds Number for experiments with Bileaflet valve. Regression performed is linear. 

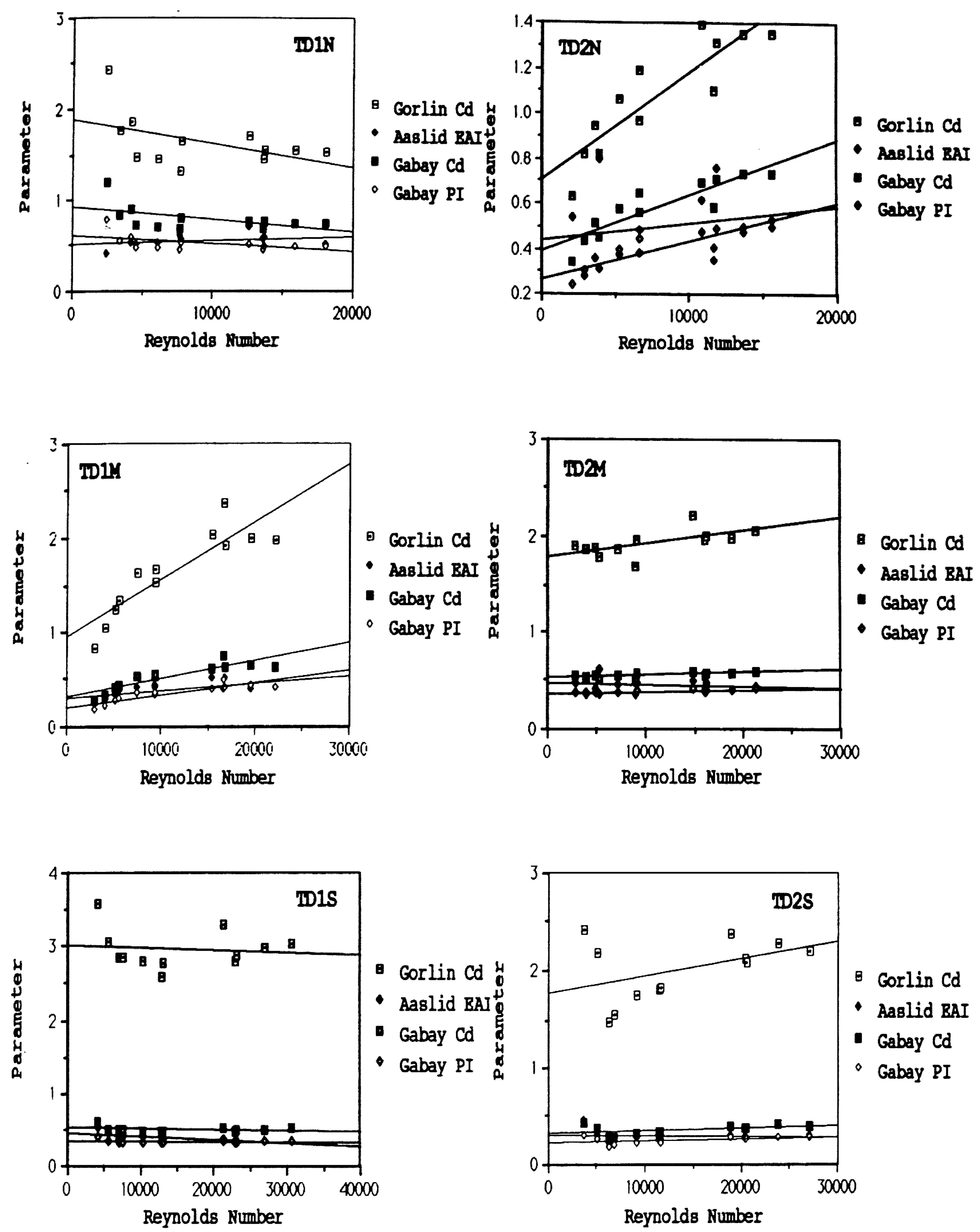

Figure 42: Four performance parameters are plotted as a function of Reynolds Number for experiments with Tilting Disc valve. Regression performed is linear. 


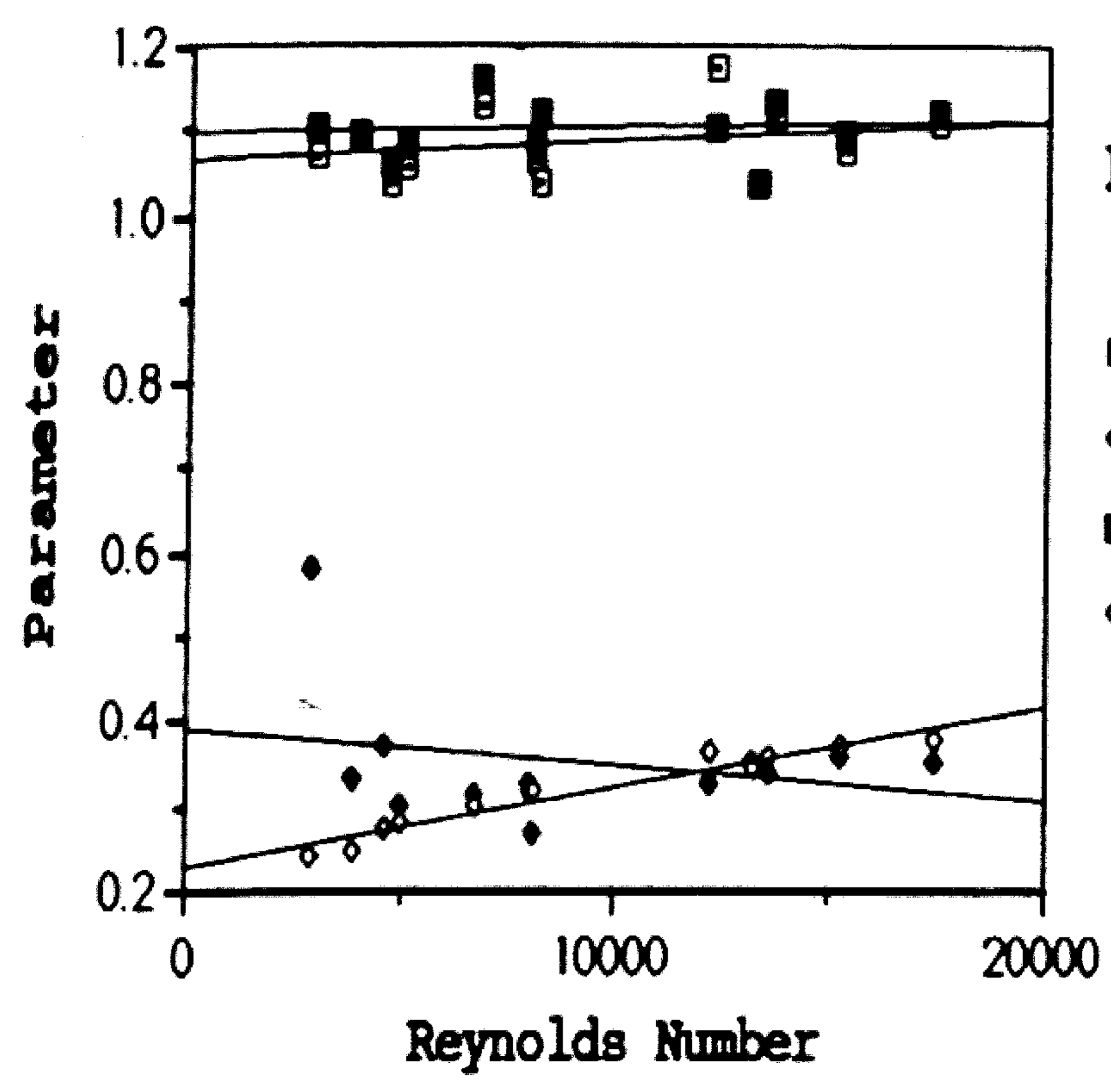

BPIN


Figure 43: Four performance parameters are plotted as a function of Reynolds Number for experiments with Bio Prosthetic valve. Regression performed is linear. 

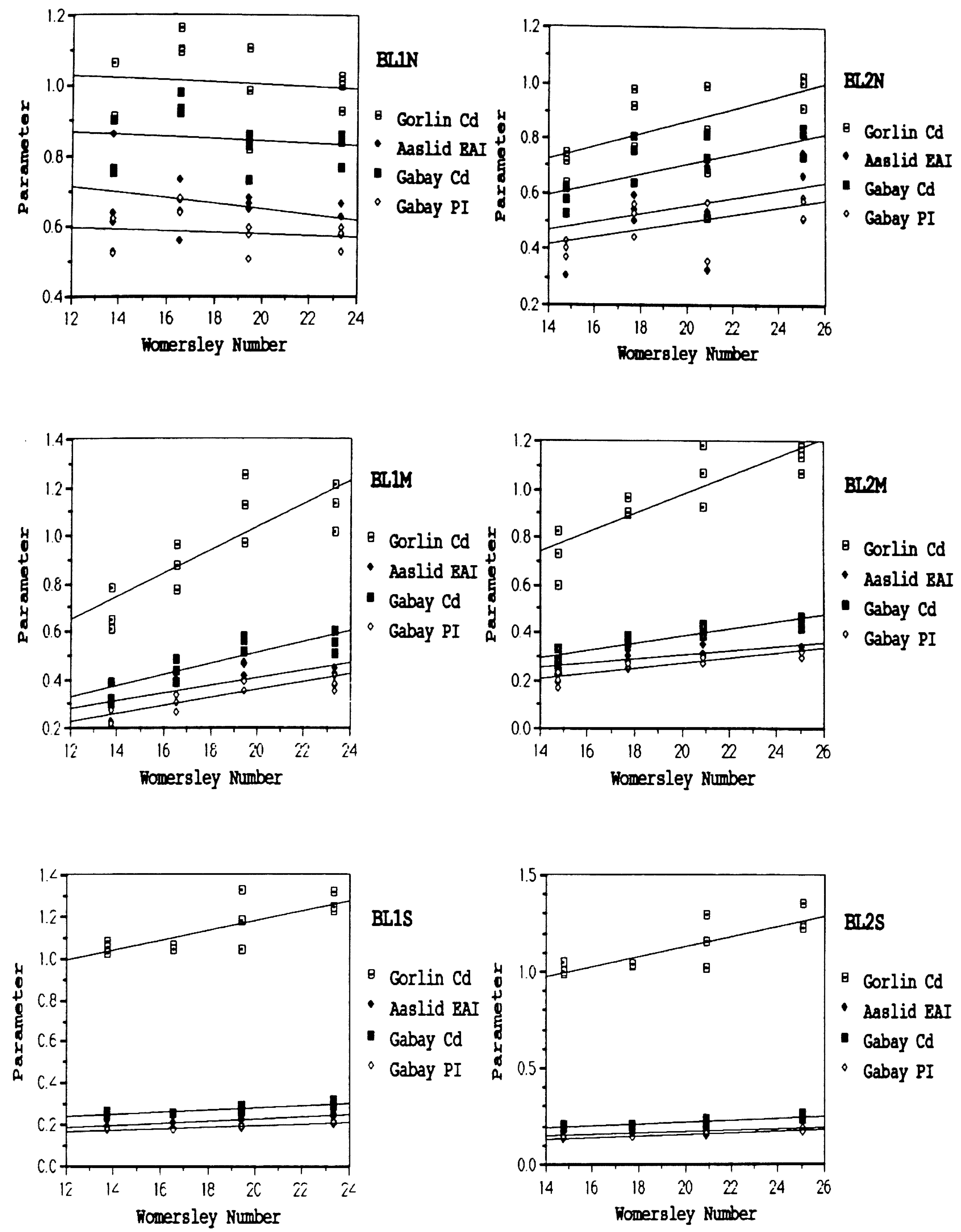

Figure 44: Four performance parameters are plotted as a function of Womersley Number for experiments with Bileaflet valve. Regression performed is linear. 

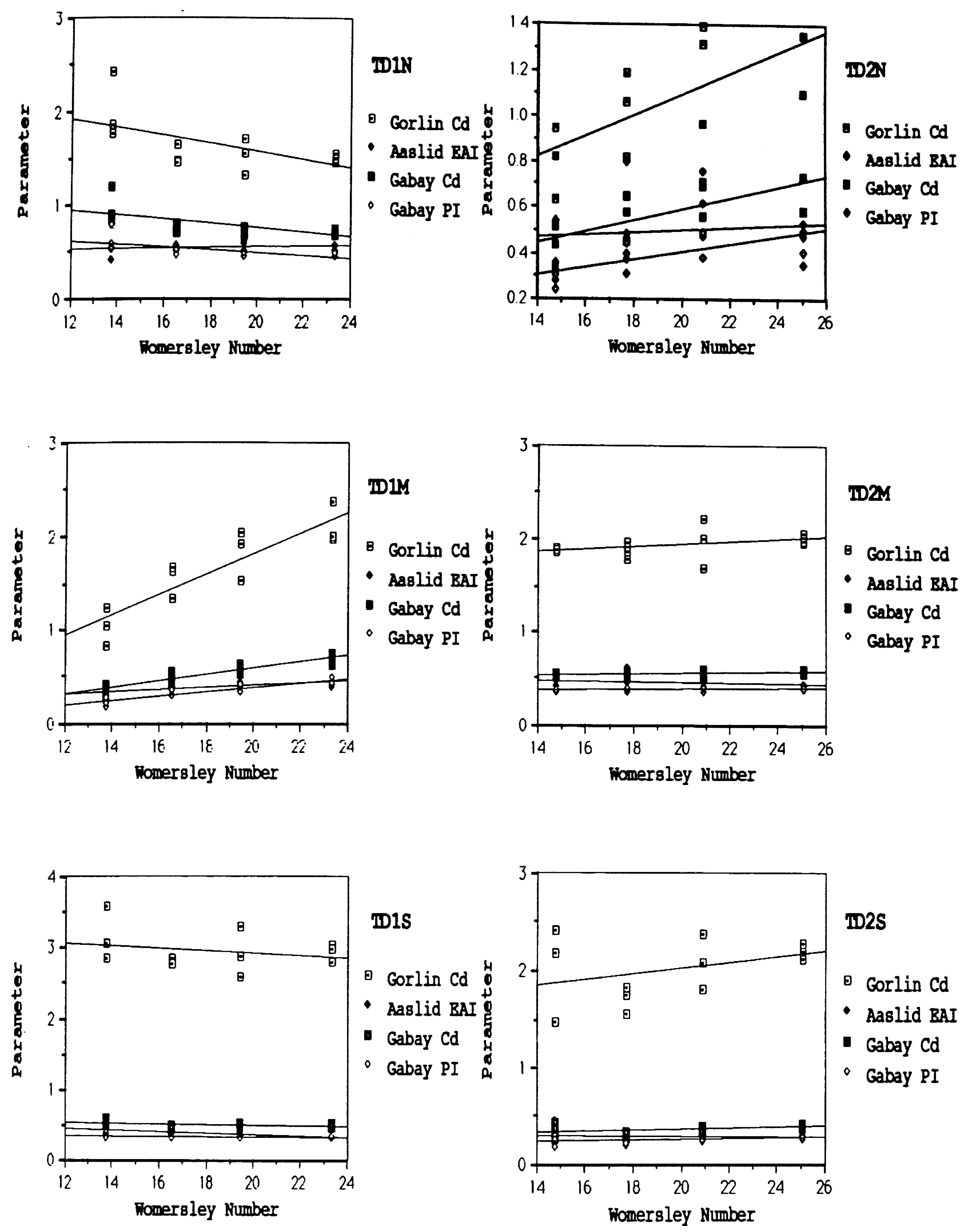

Figure 45: Four performance parameters are plotted as a function of Womersley Number for experiments with Tilting Disc valve. Regression performed is linear. 

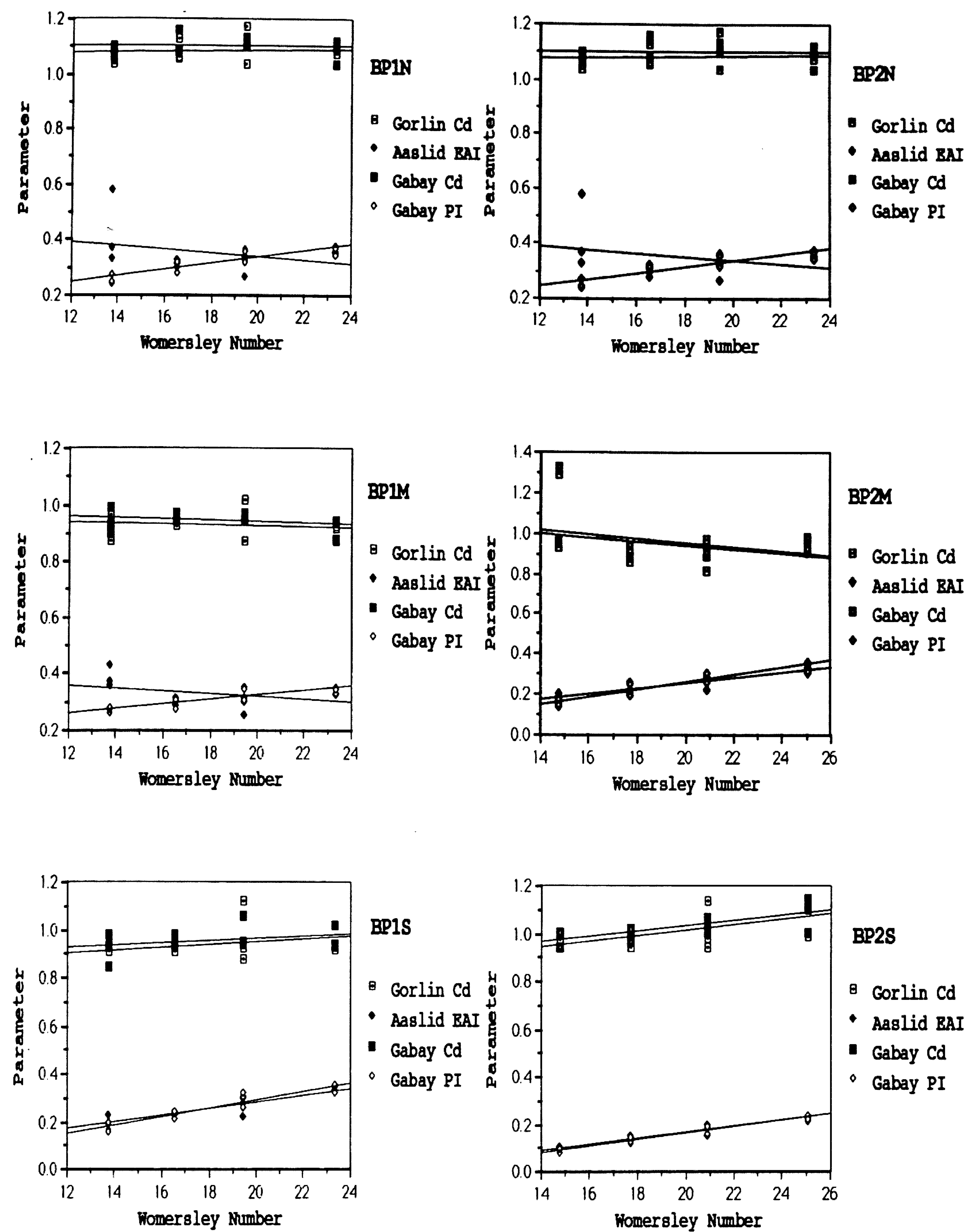

BP1S

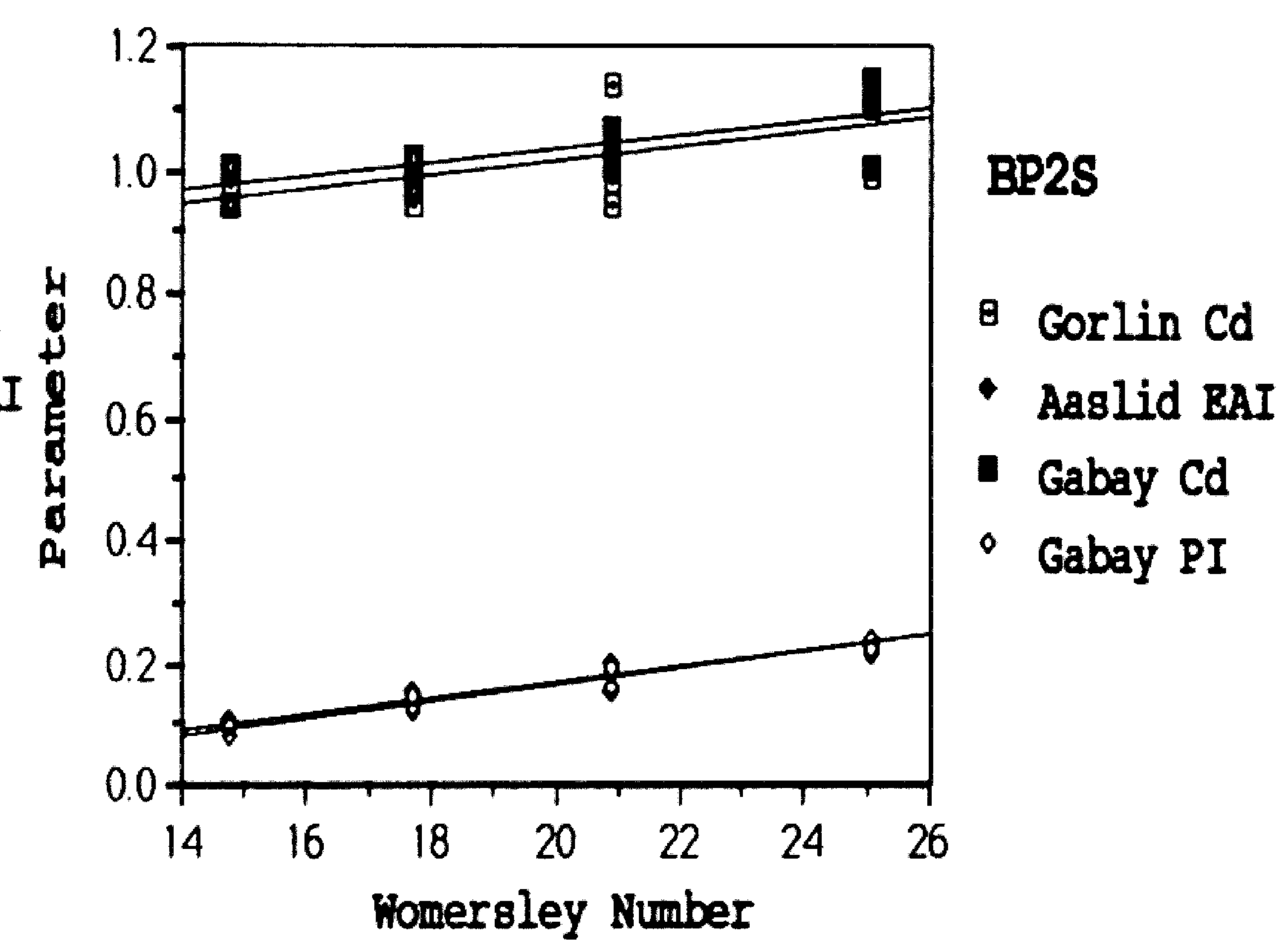

Figure 46: Four performance parameters are plotted as a function of Womersley Number for experiments with Bio Prosthetic Valve. Regression performed is linear. 

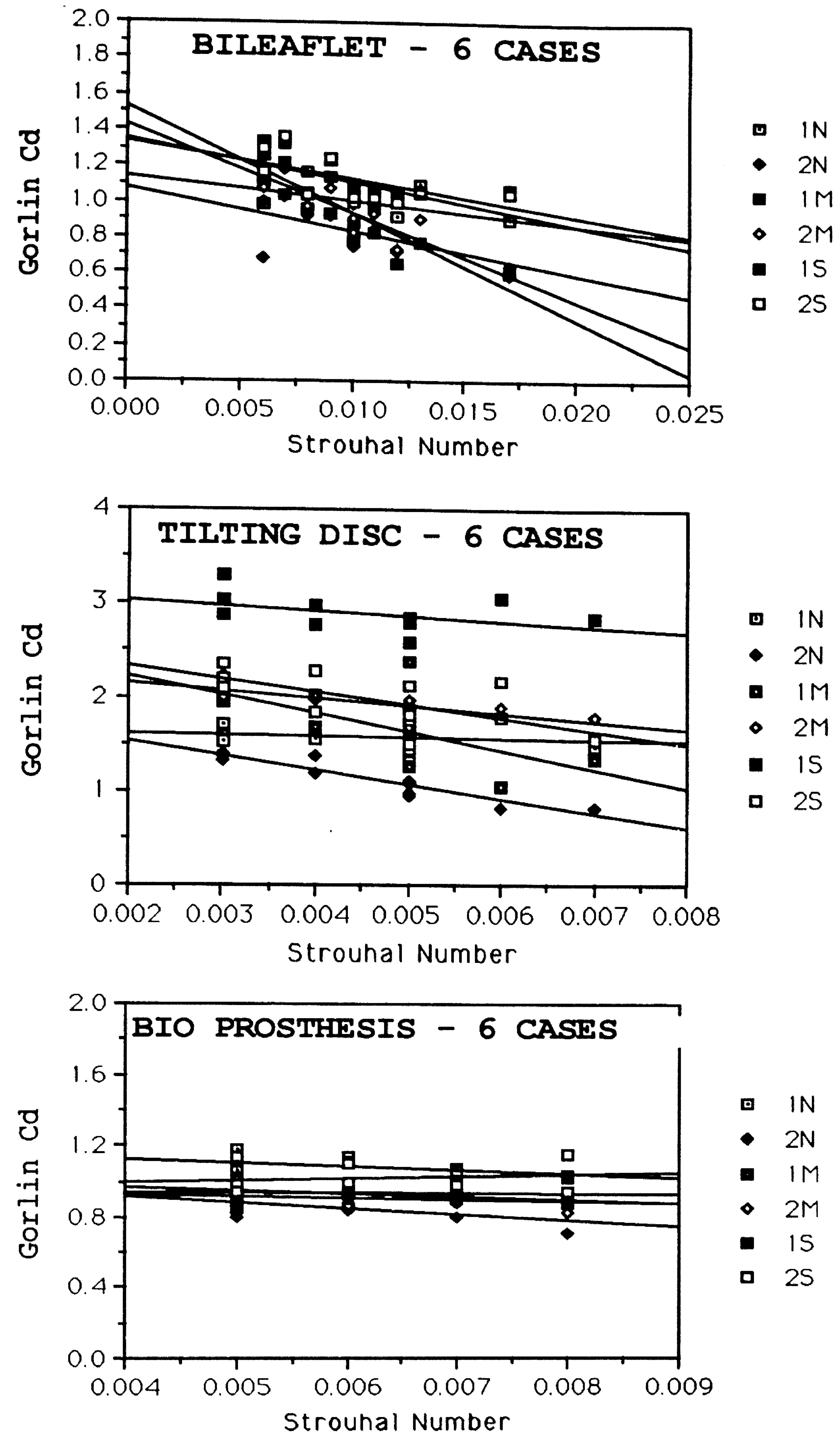

Figure 47: Gorlin Cd is plotted as a function of Strouhal Number for experiments with 3 types of Valves. Regression performed is linear. 

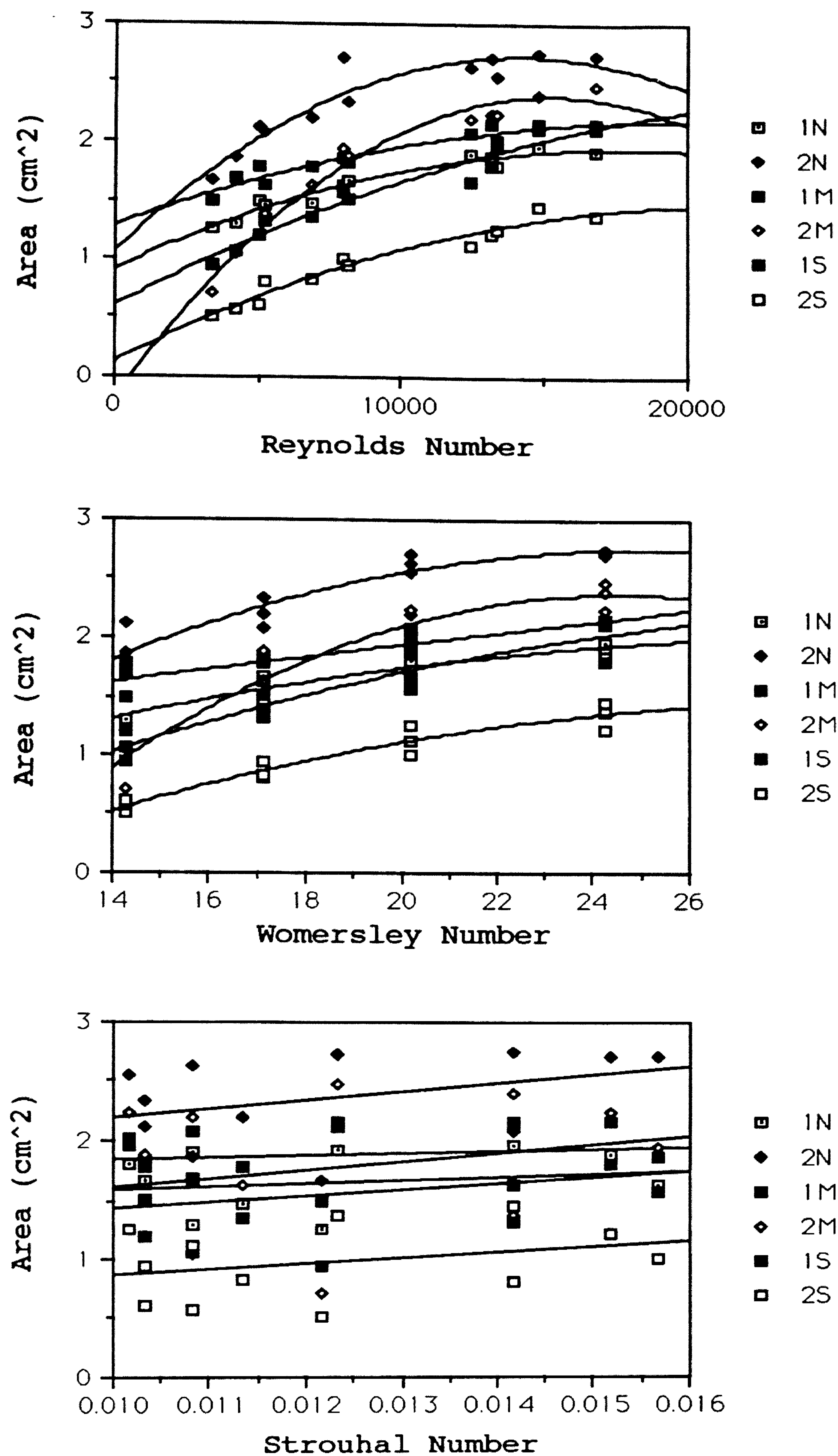

Figure 48: Effective areas for Bio Prosthetic valves are plotted as a function of each of three dimensionless numbers. 

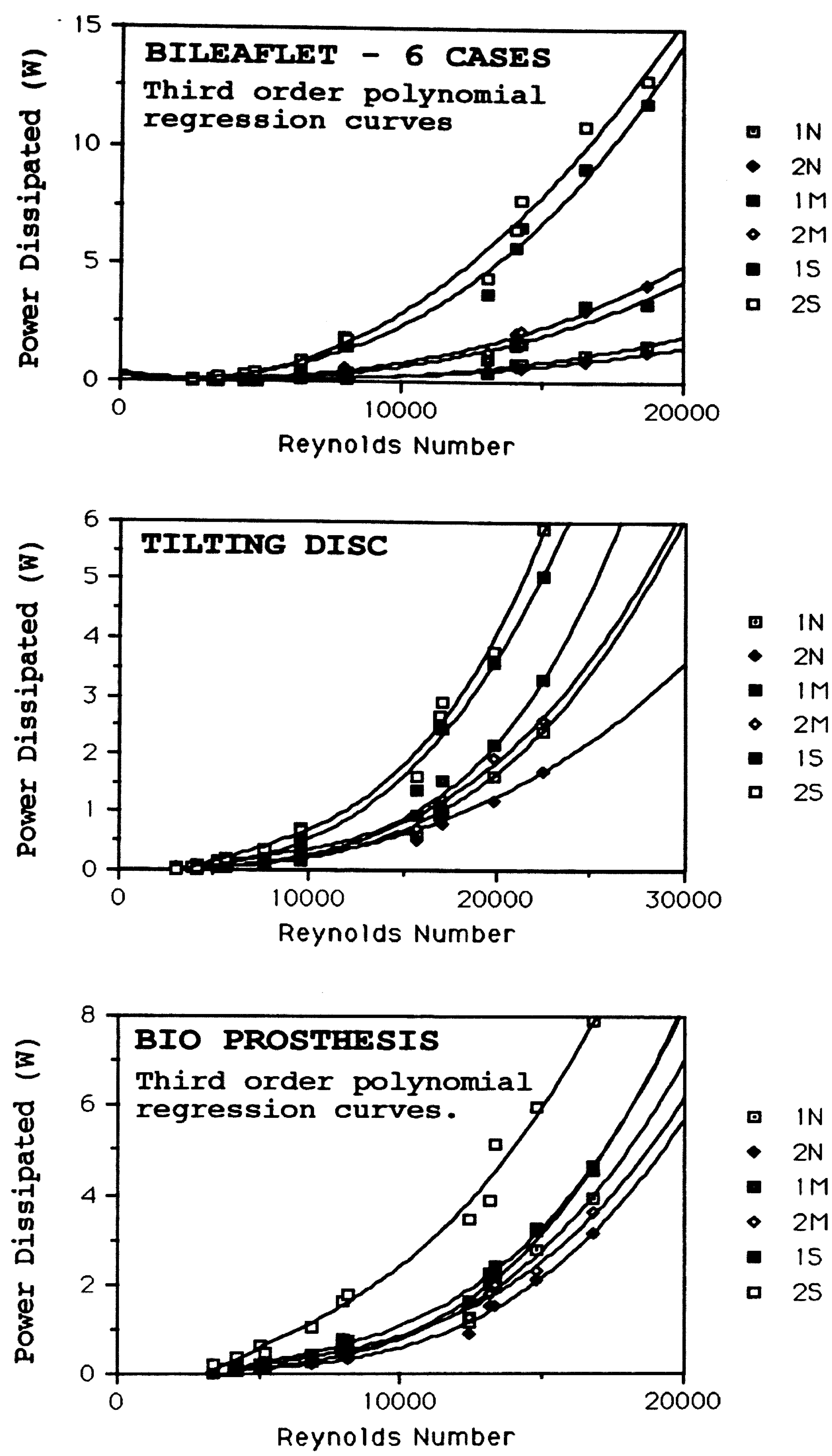

Figure 49: Power dissipated in three types of prosthetic mitral valves plotted as a function of Reynolds Number. 
In discussing the results of the experiments carried out, one must keep in mind what it is that is being sought in order to draw the relevant conclusions.

The main purpose of this part of the project is to determine what value, or values, should be used for a coefficient of discharge when assessing the performance of a heart valve with the effective area method. This determination must be dual in nature: the specific magnitudes of the Cd as well as the range for which said values are valid must be specified.

Observing figures 36 and 37 , one sees that the coefficient of discharge varies greatly, with increased, and also for a given, Reynolds number. Hence it is immediately concluded that no single line, no single linear variation of coefficient of discharge as a function of the Reynolds number can be established that applies for all valve types, sizes and operational conditions.

Next, figures 41,42 and 43 are observed. These figures show the four performance parameters plotted as a function of the Reynolds number; unlike figures 36 and 37 , however, the three former figures depict a different plot for each valve type, size and operational condition, such that each of the 18 study cases are plotted separately. If it can be established that a good correlation exists for the $C d$, as a function of 
Reynolds number, for each of the 18 cases separately, and, having shown that no such correlation can be found that applies to all cases lumped together, then, once a criterium of error is established, cases that present similar (within the error criterium) values for the $\mathrm{Cd}$ can be lumped together and the envelope of application for each Cd is established.

It is most important, if the EOA method is to be successful, to be able to determine Cd relations that apply to any condition of valvular operation, even if a different relation has to be used for each valve type and size. This claim is evidentiated by the fact that, whenever a prosthetic heart valve has been implanted, its type and size are known, whereas its operational condition is not, for that is what is being sought. Hence, a different relation for cd could be used for each valve type and size, as long as it applies across the range of operational conditions. Since only prosthetic heart valves were used in this study, the results obtained here cannot necessarily be extended to the study of natural valves, for which the exact size and configuration are not known a priori, even though the same method of research would apply to such cases. If, on the other hand, for a given prosthetic valve, no single relation can be found for the coefficient of discharge that applies to all valvular conditions, then the Cd cannot be reliably determined and the EOA method, whose accuracy is linearly dependent on the accuracy of the Cd, lacks validity to that same extent. 
Before the range of conditions for which a simple linear function for the coefficient of discharge (a function because it depends on the Reynolds number, at least) can be determined, it is necessary to establish the criterium for correlation. In other words, the maximum variation between the values of the Cd for two different study cases that can be tolerated, while considering that a single value of the cd applies, to both cases, has to be determined.

One way to carry out such analysis is to establish the total error that can be accepted in the final computation of the effective orifice area. For the purpose of this study, it will be assumed that a calculated area within plus-or-minus 108 of actual is acceptable. While in engineering terms, an error of $10 \%$ for a calculated performance parameter is considerable, in the medical sciences and physiology estimations within 1008 of a given value may be the best available. In this method, one can use standard error propagation computation to determine the effect of the errors encountered in the simplification of the correlation for the coefficient of discharge on the final computed effective orifice area of the valve.

Another way to find the operational cases for each valve that can be described by a single cd function is to lump together the Cd data for the cases in question and perform linear regression analysis on the data group. In this case, the quantitative indication of the quality of the fit is the 
regression factor (R-factor). The $R$-factor is as much a function of the slope of the regression line as it is a function of the line's ability to describe the data population. Plotting several data groups together for valves whose Cd has a low dependency on flow rate, as is the case with the tissue valves, will produce a low R-factor; and if the R-factor is the criterium whereby the quality of the fit is judged, possible correlation might be disregarded not because of he merit of the fit, but because the slope of the line is low.

Cases for which the regression factors are low because the slope of the line is low can still have their fit analyzed in terms of a simple average and standard deviation. As the reader observes, the analysis of the data generated by these experiments is complex if one wishes to draw meaningful conclusions about the behavior of the coefficient of discharge, for both regression and statistical analysis must be performed, and a 'significance' criterium has to be adopted. In addition, the analysis can be repeated in two dimensions: the Cd's dependence on both flow rate and pressure drop can be investigated; such is the topic of future work, however.

In view of the considerations offered above, the discussion of the results derived from the experiments proceeds as follows. The first line of error, that incurred in the Cd's linear dependence of Reynolds number, is investigated 
grapho-statistically in the following manner: two lines that are parallel to the regression line are drawn on the graphs such that each includes all but the furthest point away from the central line; one is drawn above the regression line, the other bellow. Since there are 13 data points per curve (13 flow rates) and 2 are being neglected (the highest and lowest) we have a significant sample of $85 \%$ of the population, (11/13) such fraction is considered adequate for this type of investigation. Next, the $y$-intercept values (the slopes are identical by definition, since the lines are parallel) for all three lines is noted and the percentual differences between the high, and low, lines and the regression line, are noted to be the error incurred simply by the description of the Cd as a linear function of the Reynolds number. This error represents the difference between what is theoretically correct and what was found in the experiment, for it is known that the Cd has to be a linear function of the Reynolds number (for each of the 18 cases) because the pressure drop increased with its second power, as observed on figures 38,39 and 40; and heavily corroborated by the literature. Details of this analysis are given by Schoephoerster. [15]

Two things become immediately apparent when this analysis is carried out: the errors determined in the fashion described are all large, varying between $2 \%$ and $25 \%$ for the BP1N and TD1M cases, respectively. Additionally, it is observed that the slope of the line is negative for three cases: TD1N, TD1S 
and BP2M. The reason for this is the inclusion of the thirteenth data point in the regression computation. The thirteenth data point was described earlier as the point that represented cardiac operation during the hours that follow valvular replacement. In general, it was found in these experiments that the Cd value for that input function, for all cases was significantly higher than the mean Cd for the same case. Since this input function imparts such a short stroke volume, and since its operational frequency is so high, it is speculated that either pressure drops measured were lower than actual, or the flows developed through the valve were smaller than expected. It is also feasible that the valve never opened fully, but that would not affect the value of the Cd since the areas used were the same for all experiments that used mechanical valves, and measured for the tissue valves, as previously explained. Since this data point produces a high value, at a low reynolds number, it is evident that its inclusion in the regression computation will shift the slope of the curve downwards. If the slope would have been nearly zero were it not for the 13 th data point, it may reach negative values with its inclusion as observed in these examples.

At this point, it can be concluded that, if the assumptions about the cardiac operational parameters (table 1) are correct for the post-surgical condition, then the assessment of valvular performance would have to wait after 
surgery until normal cardiac function resumes. This is postulated because all of the values for the performance parameter, calculated by the techniques herein described, for the post-surgical case were greater than '1'. Such is obviously not possible, for it would represent the creation of energy by the valve.

The same condition, Cds larger than ' 1 ', justifies the rejection of the variation of the EOA method where the projected open area is used for the determination of the Cd, the Gorlin formula. Strict application of the equations of continuity and conservation of mass, as used in the derivation of the Gorlin method, require that the cross sectional area be multiplied by the normal velocity vector in order to obtain the flow rate. This requirement disqualifies the measurement of the area as the projected, because the projected area is on a plane parallel to the valve's base, which is perpendicular to the main flow stream, but not perpendicular to the flow through the true orifice. It must be borne in mind that, although Gorlin applied the hydraulic principles described here to the estimation of valvular area, his main goal was the determination of the magnitude of cardiovascular shunts, (septal defects such as the Foramen ovale are among these) for surgery planning. The implication is that all of the applications of the formula, as well as its origin, presupposed an orifice of nearly circular geometry through which fluid passed. This was in 1951. Since then, roughly from 
the mid-1960's, mechanical heart valves became available in which some form of occluder is at the center of the orifice, around which, in an annular fashion, the fluid flows. There is no reason to suppose a priori that the formulations described earlier would no longer work for these geometries. However it must be recognized that the primary flow through the valve is no longer, necessarily, perpendicular to its base; instead, it is parallel to the leaflets while flowing through the valve. The convective acceleration necessary to cause such change in directions is thought to consume more energy, other things being equal, than would be required if a central-flow valve configuration was employed. This discrepancy becomes more pronounced as the degree of stenoticity of the valve increases because, as is seen on figure 8 , the opening angle of the valve decreases, causing a greater radial acceleration of the flow and increasing the difference between the projected and the true orifice areas.

The difficulty with the measurement of the projected area is more pronounced in the case of the monoleaflet valve because, having a larger occluder, a greater radial acceleration is imparted on the flow, and, due to the nature of its configuration, a greater difference between the observed and actual flow areas exists. The effect of this difference is that projected areas much smaller than the actual perpendicular-to-flow areas will be used in the calculation of the Cd. Since, for a given flow rate and 
pressure drop, a smaller opening area is indicative of a larger coefficient of discharge, if enough errors exist, the Cds can appear to assume values much greater than unity, which, again, is a physical impossibility. This shortcoming can be completely eliminated by the use of the primary flow (FA) or the mounting area (MA), such as Aaslid and Gabbay do, in the determination of the $\mathrm{Cd}$. Such formulations, however, require the use of flow measurements which are not commonly available clinically. Nonetheless, the results obtained from the Aaslid and Gabbay formulas show Cd values which are much more physically meaningful.

The significance of these different definitions of areas is interesting: other things being equal, the use of the mounting area in these equations becomes a measure of how spatially efficient a valve's sewing ring is; the use of the primary flow area becomes a measure of the valve's occluder geometric design and range of motion; the use of effective area becomes a measure of the merit of the valve's primary configuration, which in this case was either of the centralflow or central-occluder type.

Even though the magnitude of the Cds associated with some of the valves is not physically meaningful, the error observed is constant throughout the range of experiments, at a given condition, with that valve. This situation limits the use of the Gorlin formulation considerably, since the difference in the projected and actual areas increases non-linearly with 
increased simulated stenoticity.

The average error in the linear correlation of Cd with Reynolds number was 118,178 and 68 for the 6 cases of the BL,TD and BP valves respectively. Figure 50 shows the mean value of the cd when lumped together by valve type, size or condition, as well their correspondent standard deviations. It is observed on figure 50 that the lowest standard deviation shown corresponds to the Bio Prosthetic valve (Sd=0.103, mean=0.92) which means that the Cd for this type of valve varies the least with the Reynolds number, valve size and condition. The highest standard deviation, on the other hand, is observed for the severely stenotic group of valves, $S d=0.751$. Judging from the mean value of the $\mathrm{Cd}$ in this instance, 1.5, and the mean value for the $\mathrm{Cd}$ of the tilting disc valve group, it is apparent that the latter strongly influences the former. If the TD group is removed from the calculations, the new standard deviation for the severely stenotic group becomes actually the lowest of all groups after the Bio Prosthetic case; $(S d=0.126)$ which allows us to correctly infer, as confirmed by the plots of figure 43 , that the Cd for the BP severely stenotic valve is the least dependent on size.

It can further be concluded, overall, that for low Reynolds numbers a single Cd correlation applies for the $29 \mathrm{~mm}$ Bileaflet valve in the normal and mildly stenotic conditions. For the $27 \mathrm{~mm}$ size, the same can be said for the normal and 
severely stenotic conditions. At high Reynolds numbers the Cd values become more uniform between the two sizes and among the three conditions, with the exclusion of the $27 \mathrm{~mm}$ Normal valve, for which no good correlation was found.

For the Tilting Disc valve a qualitative correlation is only observed between the $29 \mathrm{~mm}$ mildly and severely stenotic cases. Finally, for the Bio Prosthetic valve, excellent correlation was found between the two sizes of the severely stenotic case, as alluded to earlier.

Upon closer investigation of what at one time seemed to be physiologically accurate, it is discovered that the wide range of conditions studied could never occur within a human cardiovascular system. Specifically, while each component of the study, such as flow rates, valvular condition and pressure, was carefully designed to duplicate physiological conditions, their combination in a purely combinatoric fashion produces results which are not all physiologically feasible. Hence, while all flow rates used here are attainable, the higher values would not occur where mitral valves are severely stenotic, for instance. The reason for this became apparent when it was realized that the cardiovascular system must be optimized for the least energy expenditure. In view of this, further thought is given to the range of conditions that are expected to be observed in vivo, and, for these, cd correlations are sought after once anew.

According to Katz [33], transmitral pressure losses of up 
to 30 mmig may be observed in the most severe cases of stenoticity. Looking at figures 38,39 and 40 , it is observed that pressure $108 s e s$ of $30 \mathrm{mmHg}$ are associated with Reynolds numbers of roughly 10000. On figure 49 it is observed that at this-Reynolds number, power losses in the valve range fron less than 1 to 2.5 Watts, depending on the valve and case in question. Imposing a power-loss of 2 Watts and a pressure drop of 30 mmig limitation on the population of data points obtained from these experiments, figures 51,52 and 53 are generated. These figures show the Gorlin Cds the survived the cleaning described plotted as a function of Reynolds number (a) and transmitral flow rate (b). In both cases, for all three figures, it is observed that the correlation factors are still quite low, indicating the lack of correlation with the ordinate, size or condition. Since it was already demonstrated that the correlation with the ordinate must be linear, figures ' $a$ ' and ' $b$ ' are broken into their components: 27 and $29 \mathrm{~mm}$. since figures ' $c$ ', ' $d$ ', ' $e$ ' and ' $f$ ' also show very low $R-$ factors, it is concluded that no Cd relation that applies for all valvular conditions exists.

The above analysis can only be carried out in a rough qualitative way, for numerically, no meaningful correlations were found. Although this research proposal was originally accepted to extend only to the point where a final word on the EOA could be achieved, the large variability of the results obtained, as well as the fact that the EOA method does not 
account for valvular regurgitant losses, compelled the author to derive and propose another possible method of estimating valvular function, one that is thought to be more physically correct. The result of this endeavor is the Energy Index method.

Errox considerations

The purpose of this section is to expose some of the sources of errors encountered in this work, as well to offer a few suggestions for future work intended to reduce errors.

In principle, errors that would be encountered while applying the EOA method in vivo are higher, necessarily, than those incurred in the controlled and repeatable conditions of a laboratory experiment.

clinical estimation of the effective orifice area, which is limited to the Gorlin method, is based on measurements of flow rate and pressure drop through the valve. since the advent of catheter mounted piezoelectric pressure transducers, pressure measurements in vivo have become much more accurate than was possible with the fluid-filled manometer. Although incorrect placement of the catheter tip could cause incorrect pressure measurements, if such procedural shortcomings are avoided, measured pressures can be very close to actual. With the sensitivity of the instrument at 5 microvolts per volt per millimeter of mercury, and with the use of an analog to 
digital converter operating at $4 \mathrm{Khz}$, measured pressures have to be well within 18 of actual.

Clinical measurements of transvalvular flow rate is done in a more indirect fashion than pressure measurements since the only true flow meters that exist, such as the electromagnetic and the paddle wheel, for instance, are highly invasive, thus not finding justified clinical use unless during surgery, while the thorax is already open anyway, but when it is already too late to assess the performance of the valve.

The practical alternative to the estimation of cardiac output is any variation of the diffusion principles established by Fick in 1870. Thus, the oxygen consumption divided by the arteriovenous oxygen concentration difference is the blood flow. Oxygen consumption can be determined with a. gas analyzer that measures the difference in oxygen concentration between collected samples of inhaled and exhaled air. The additional oxygen concentration found in any of the main arteries, up from that at the venous system is determined from simultaneous blood sampling, and must be proportional, if continuity is maintained, to the amount of oxygen absorbed. The die-indicator dilution and the thermal dilution methods are based on the same principle as Fick's oxygen consumption; in these cases either the die concentration or the temperature difference are proportional to blood flow. With all of the measurements described as necessary to obtain the cardiac 
output, and only the mean output is possible, hence forcing the use of the Gorlin formula, (Out of the four formulas presented, the only one that uses the mean, as opposed to peak or RMS, flow rate) it is speculated that errors associated with measurements of flow will predominate in the estimation of the effective area.

Other sources of errors involve the valvular areas used. In these experiments, three areas were defined for each valve, following recommendations by Gorlin, Aaslid and Gabbay, as explained earlier. The mounting area is hardly subjected to error since it is just calculated based on the nominal diameter of the valve. The errors associated with the primary flow area are also small, since that is based on the measured internal diameter of the valve. A high-resolution caliper was used to measure said diameter at three different locations along the circumference of the valve, thus reducing possible errors. The effective area is the most subjected to error, since it is the product of the photography-planimetry system already described. In this method, by far, the largest contribution to the error comes from the tracing of the valvular open area with the planimeter. The worst case would be where the trace was done consistently inside or outside of the actual boundary. If the tracing error was as high as 1 millimeter to either side of the actual boundary, the error would be: 
Diameter of typical area traced: $75 \mathrm{~mm}$

Corresponding area: $4418 \mathrm{~mm}^{\wedge} 2$

Smallest traceable area $(74 \mathrm{~mm}$ dia.): $4301 \mathrm{~mm} 2$

Largest traceable area (76 mm dia.): $4536 \mathrm{~mm}^{\wedge} 2$

Errors: $(4301-4418) / 4418=-2.658$

$$
(4536-4418) / 4418=2.678
$$

Scale factor (Area of photograph/actual area): 3 to 4

Corresponding orror $(2.66 / 3.5)=0.758$

Under the worst conditions, it is observed that the measured area can only be in error, as compared to the actual area, to about $3 / 4$ of one percent, not counting other sources of errors such as the resolution of the camera and corresponding printed image.

Finally, a word is offered about possible errors found if the computed effective area is used to access the performance of valve operating in vivo. Diagnosis of valvular condition can only be done when comparing the calculated effective orifice area with a valvular area that is judged adequate for a given heart. It is my understanding that such areas have been tabulated and are based on body weight and height. The particulars of the "normal" valvular areas are best left to physiologists and anatomists; it can only be speculated here that some sort of error is introduced in the diagnosis when using these standard tabulated areas. 
Although the section on the BOA method ended on a negative note, it is suggested that further analysis of the data obtained be carried out in order to extract any possible correlations that were missed with the method used here. Specifically, it is suggested that analysis be based not on the measured effective area but on the mounting area; primarily for the reasons described above (difference between true flow area and projected area) but also because the mounting area is the value used clinically to refer to a valve. The mounting area is also unaffected by operational condition of the valve.

What has been called the coefficient of discharge in this paper cannot be determined, in strict engineering terms, by the methods described here. The engineering coefficient of discharge of the valve is not necessary for its clinical evaluation, however. Instead, it is proposed that the term "correction factor" (Cf) be used. Not being directly indicative of the losses through the valve, as a Cd is, the Cf can assume values larger than 1 .

The computation of the $C f$ would also be based on the transvalvular flow rate and pressure drop, such that the cf is described in terms of a surface instead of a line. This modification is thought to increase the range of applicability of the EOA method since the main operational difference 
between a normal and a stenotic valve is the pressure drop observed.

Finally, the author notes that, perhaps, other methods of assessing valvular performance in vivo which are not invasive may have now reached the level of technological development that justifies their use in place of the more invasive EOA method. In the case where the performances of several prosthetic valves is being compared in vitro, it is speculated that the Energy Index method, if proven successful, may be more effective than the EOA method because it accounts for regurgitant losses. 


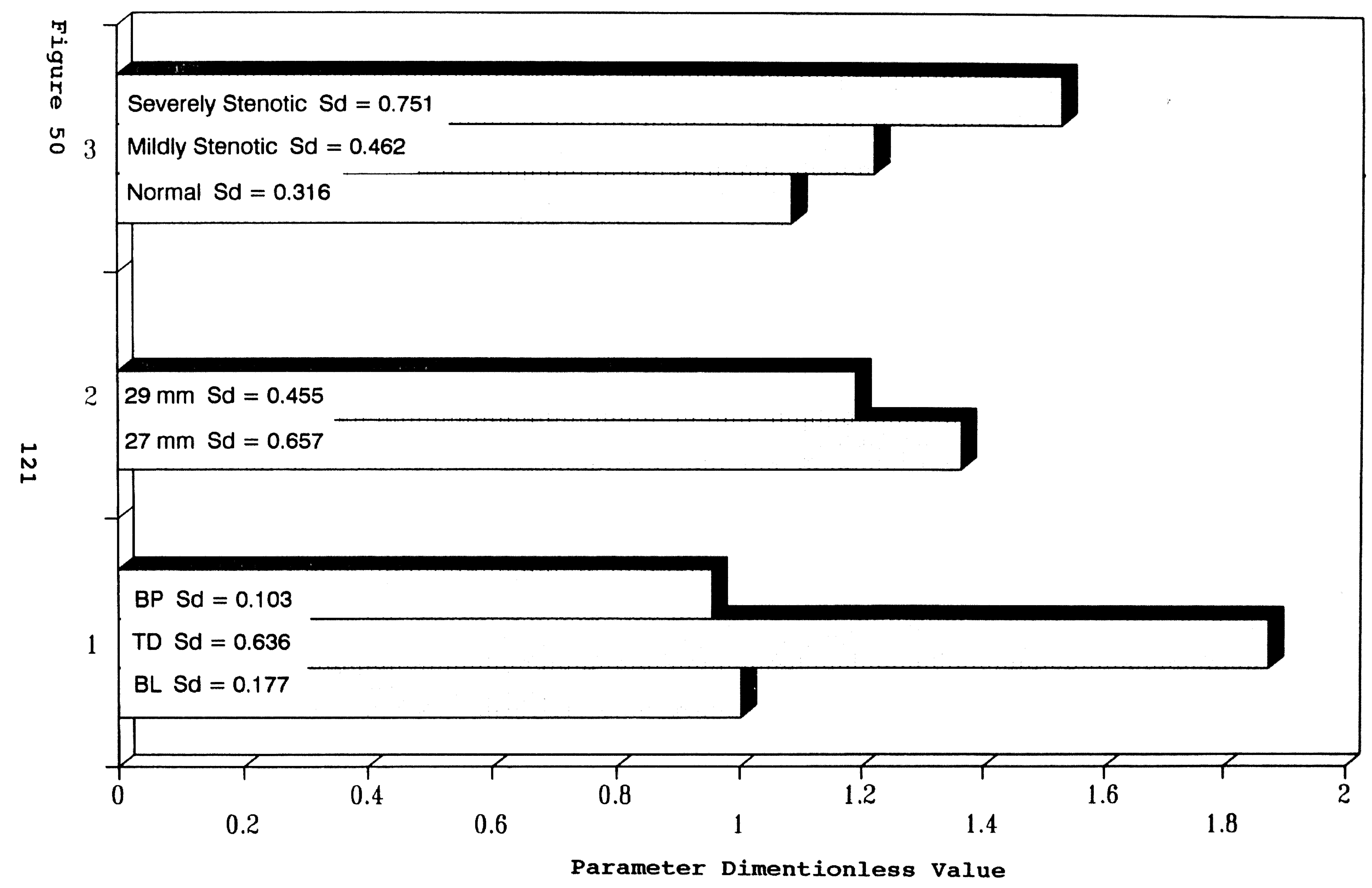



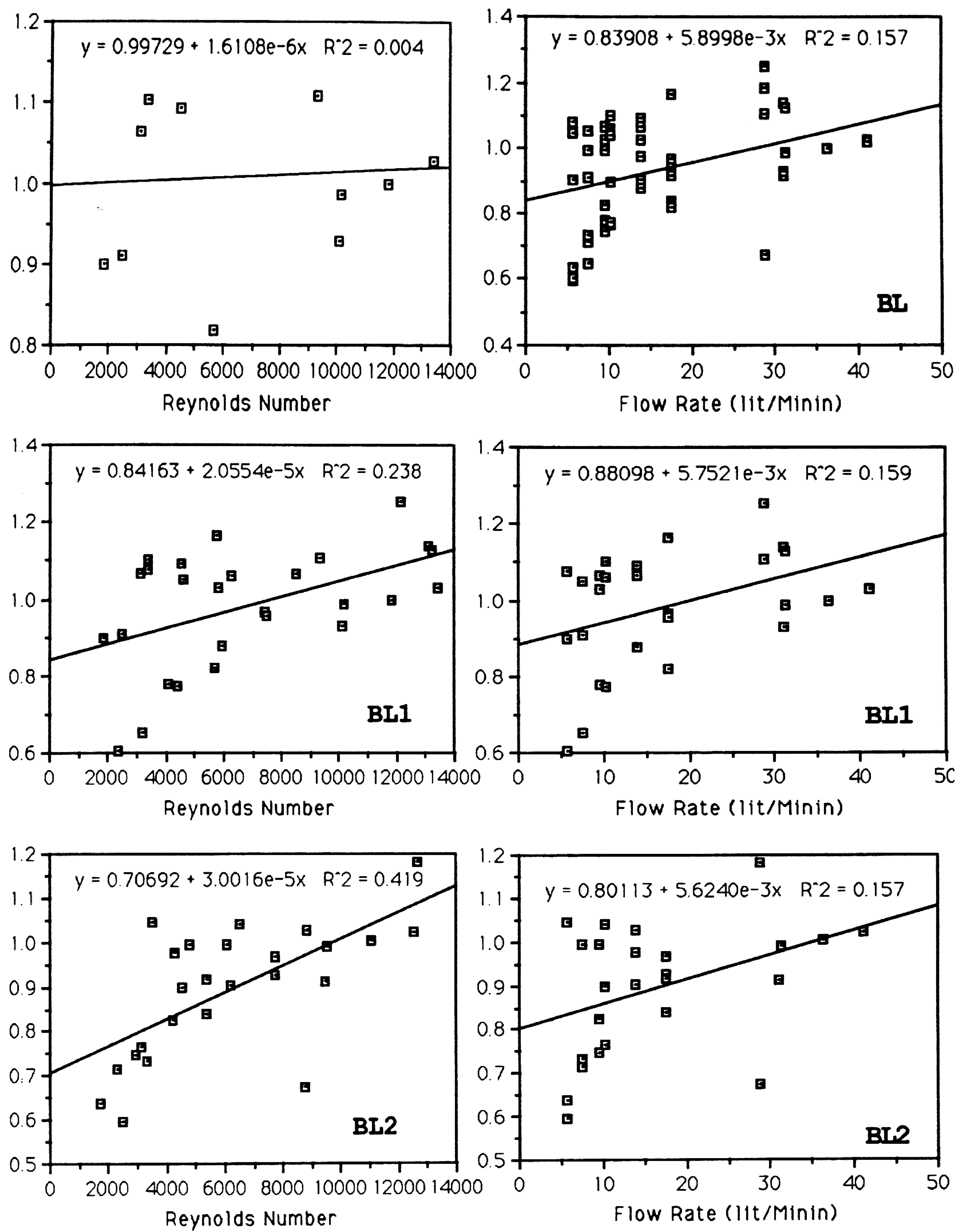

Figure 51: Gorlin Cd is plotted as a function of Reynolds Number and Mean Flow Rate. Sample was first limited to a pressure drop of $30 \mathrm{mmHg}$ then to a mean power drop of 2 Watts. 

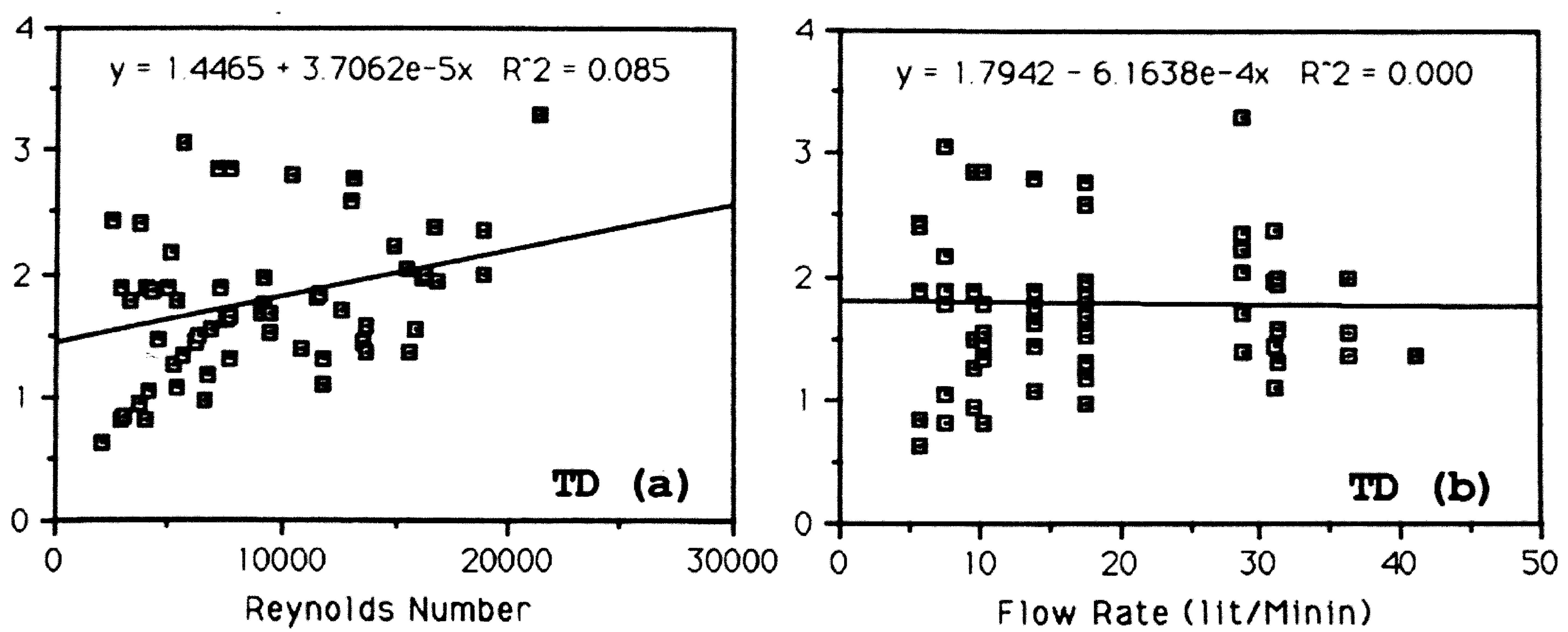

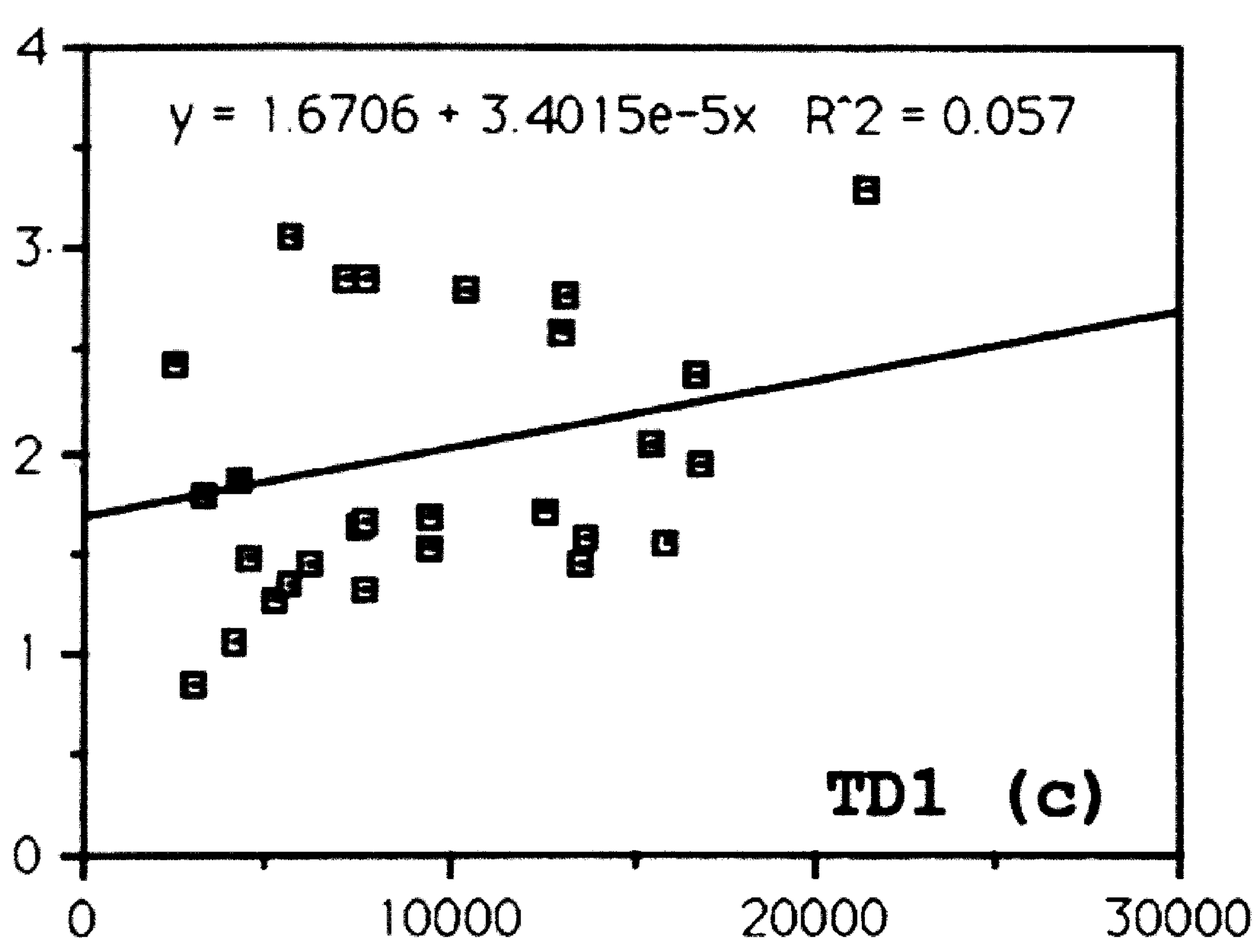

Reynolds Number

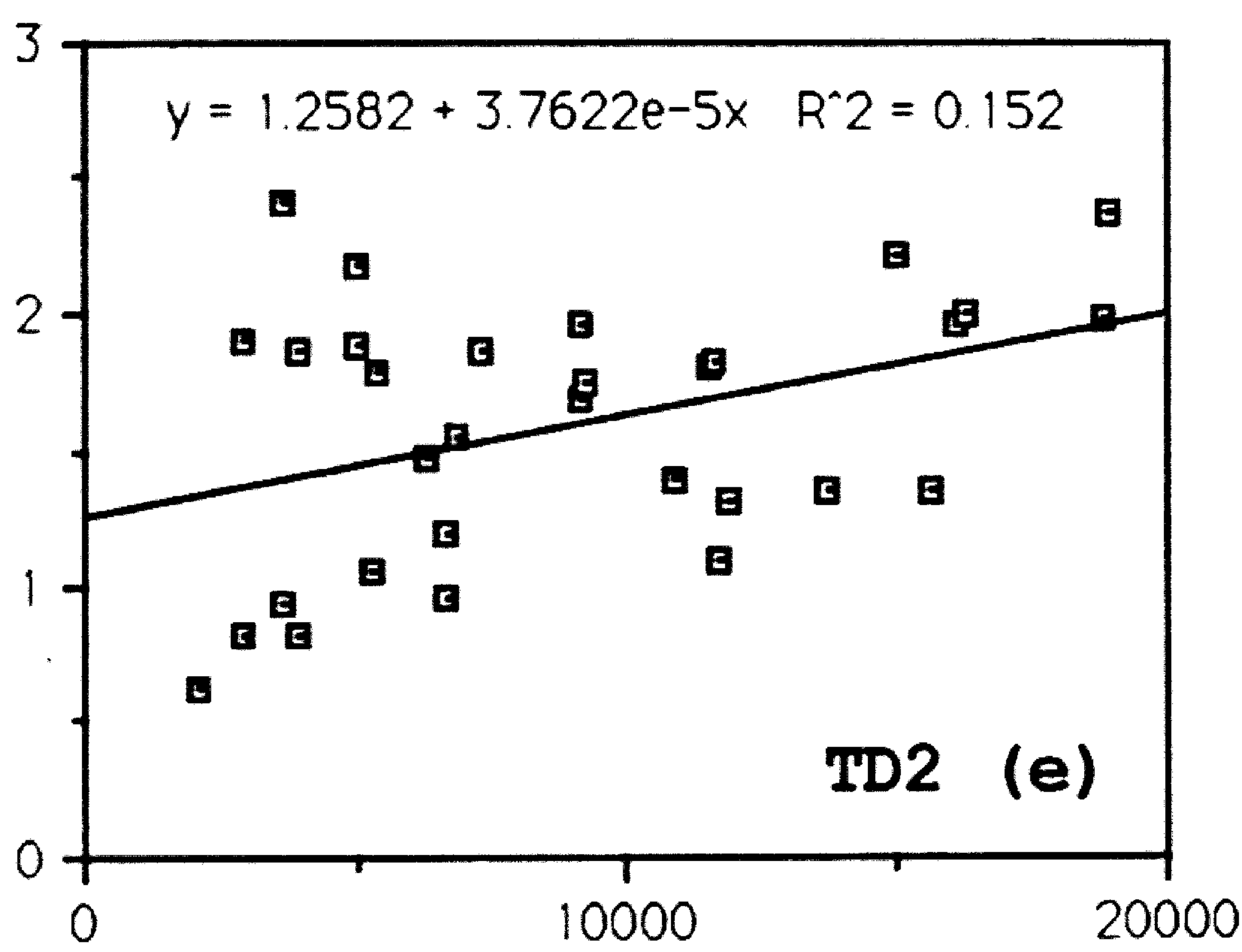

Reynolds Number

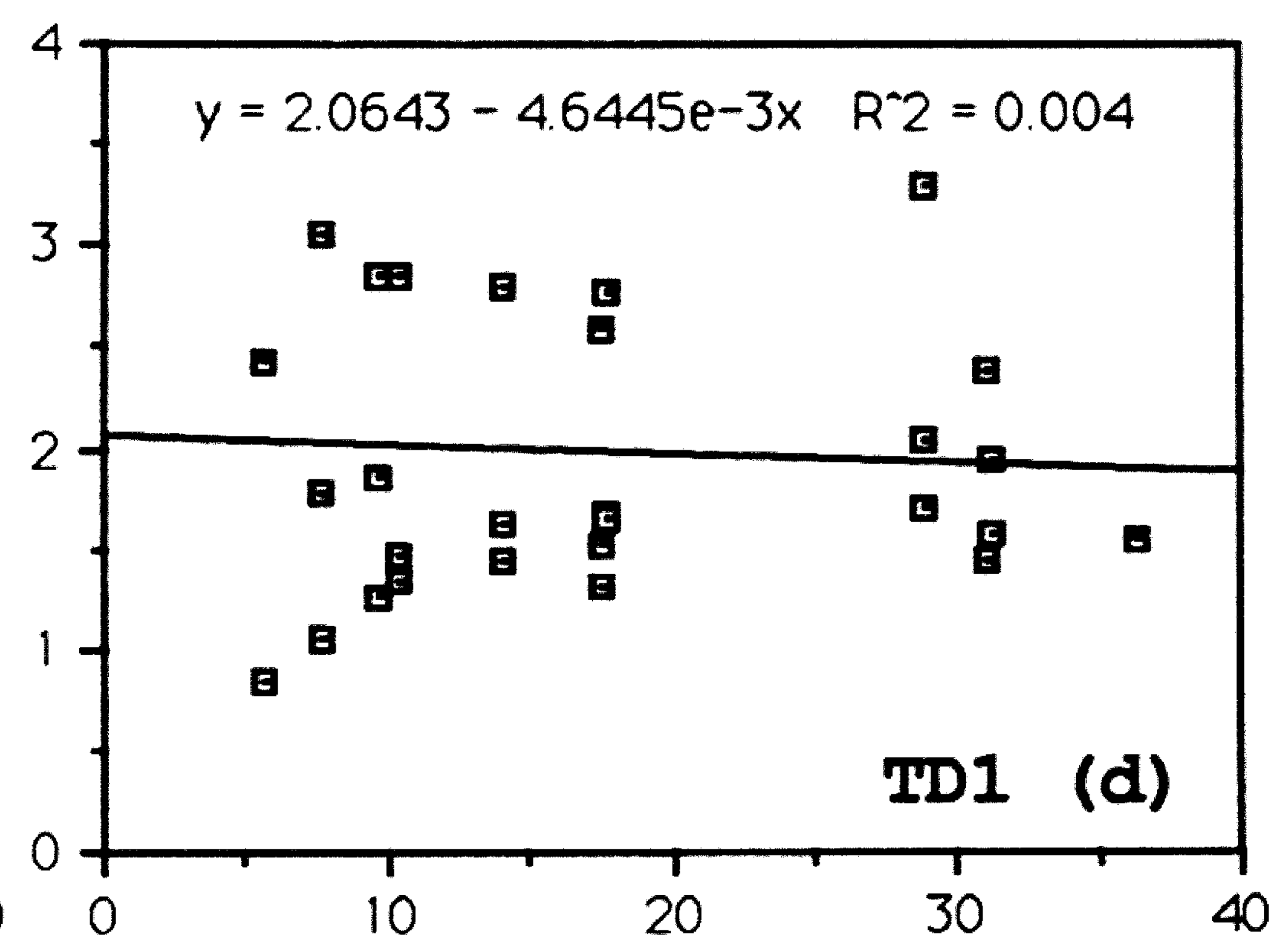

Flow Rate (lit/Minin)

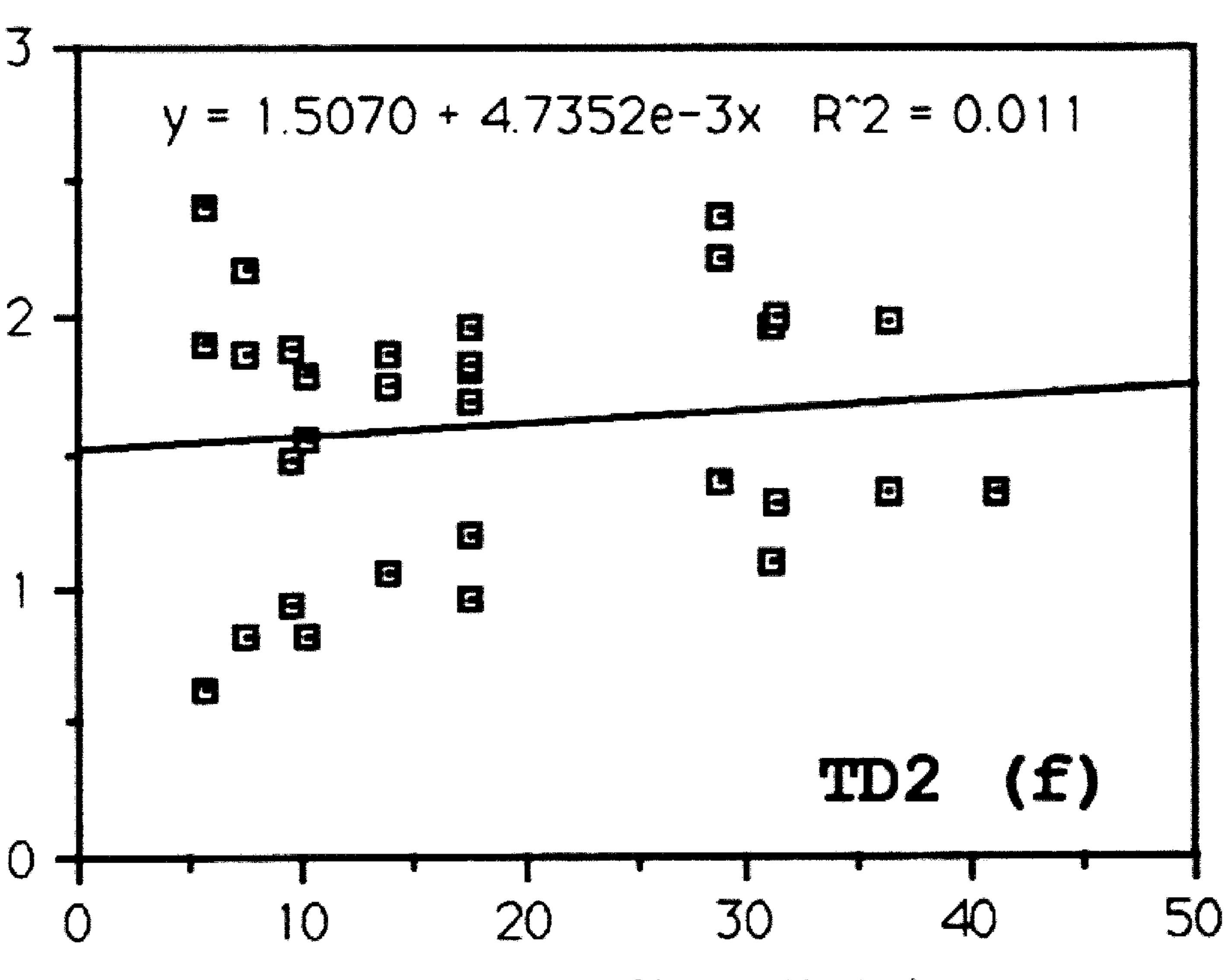

Flow Rate (lit/Minin)

Figure 52: Gorlin Cd is plotted as a function of Reynolds Number and Mean Flow Rate. Sample was first limited to a pressure drop of $30 \mathrm{mmHg}$ then to a mean power drop of 2 watts. Tilting Disc Valve used. 

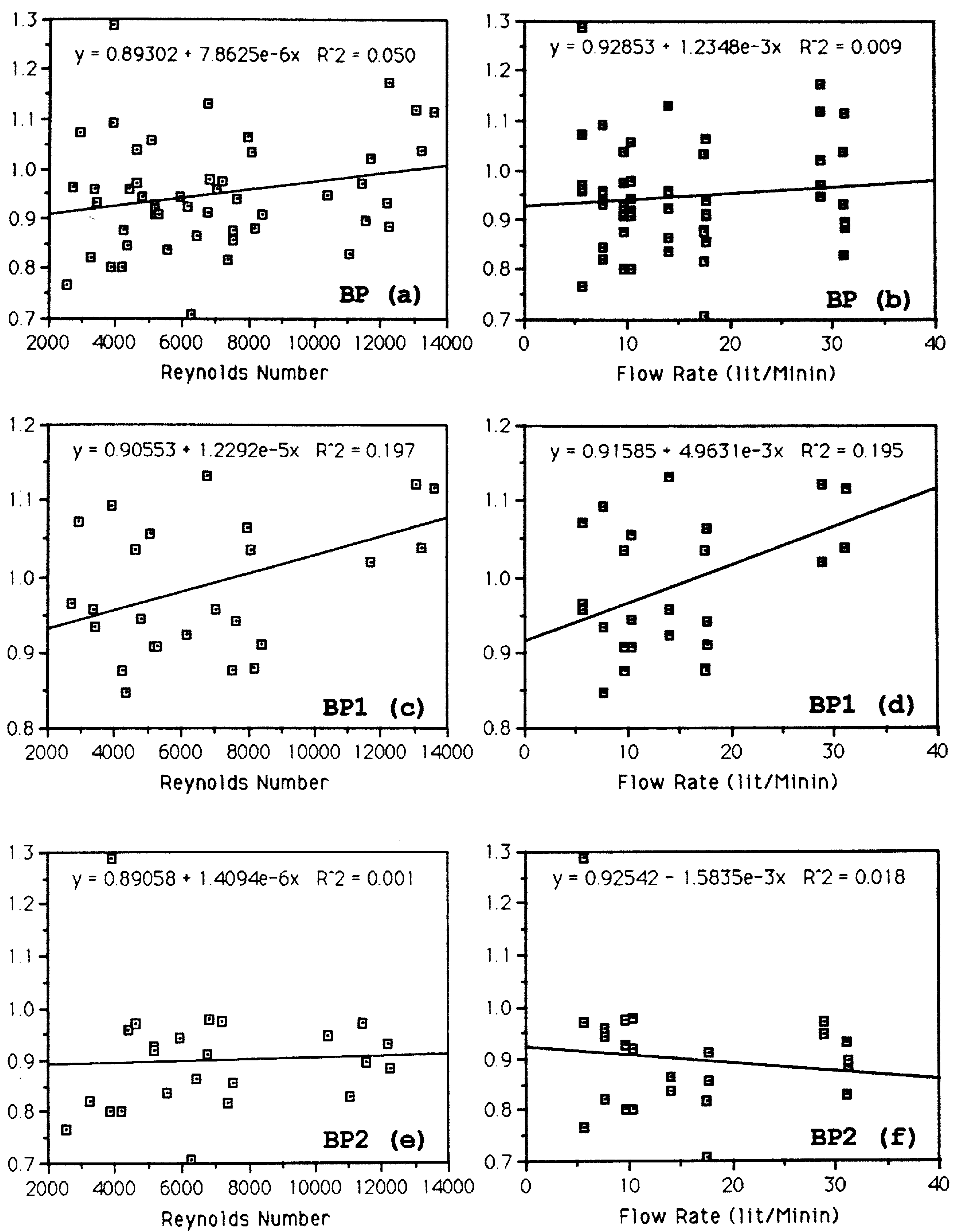

Figure 53: Gorlin Cd is plotted as a function of Reynolds Number and Mean Flow Rate. Sample was first limited to a pressure drop of $30 \mathrm{mmHg}$ then to a mean power drop of 2 Watts. Bio Prosthetic Valve used. 
Introduction

It was demonstrated in the last chapter that all orifice area methods of assessing valvular performance suffer from two drawbacks that render them nearly useless: the impossibility of determining operational envelopes for the needed coefficient of discharge, and the lack of accountability for the losses incurred in the system due to regurgitant flow (retrograde flow through a closed valve).

These limitations led to the development of the energy index, which completely addresses the shortcomings associated with the EOA method. The basic concept of the method is to determine the ratio of energy dissipated in, and because of, the valve, to the energy available for that cycle of the cardiac stroke. The energy dissipated in the valve is a measure of its characteristic during forward flow, while the energy dissipated out of the system because of the valve becomes a measure of its regurgitant behavior. The energy available for work, the denominator of the index, is the energy imparted onto the blood by systolic contraction, for the aortic valve; or the energy at the left atrium, for the mitral valve. Even though the assessment of valvular performance by energy methods gained increased attention after the papers introduced in 1987 by Leefe et al [34], and Hwang 
et al in 1988 [35], the method was still unable to account for heavy regurgitant losses; furthermore, neither Leefe nor Hwang propose a performance index, they simply describe what the losses in the valve are. The energy index method, as proposed here, to the knowledge of the author, being original, produces a quantitative dimensionless parameter that completely describe the valve's characteristics; one indication of its correctness is that, where the net flow rate through the valve is 'zero' (total regurgitance) the index will also be 'zero'.

\section{Provious morks}

Several investigators have proposed that some form of energy loss should be used to assess the performance of prosthetic heart valves. Olin, in 1971 [41], measured valvular performance as the difference in energy measured at the ventricle and the aorta. Gilbert, in 1970 [42], from in vivo data, derived some complex third degree regression equations, whose coefficients depended on valvular type and size, to determine the power dissipated in the valve. In both cases mentioned, regurgitant losses were not taken into account. Of course, at the dawn of valvular transplantation, the only important parameter to assess was whether or not the prosthetic device represented an improvement, even if only a short-term improvement, on the diseased natural structure. Later, it became evident that significant improvement on the 
hemodynamic design of valves was possible, particularly where it concerned the mitral valve, whose flow is almost entirely passive, thus being more sensitive to pressure drop resulting from poor design.

It was in 1987 that Leefe and Gentle [34] derived an appropriate governing equation for the energy loss through a heart valve. It is somewhat redundant to repeat here the work of these investigators, therefore only a brief description of the simplifications necessary to obtain the working from the governing equation is offered.

Equation (10) is the working equation, used to determine the total energy lost during one segment of the cardiac cycle.

$$
\begin{aligned}
\text { Energy Loss }= & \int_{0}^{T} \Delta P Q d t \\
\text { where } \Delta P & =\begin{array}{l}
\text { piezonetric pressure drop across the } \\
\text { valve, }
\end{array} \\
Q & =\text { volumetric flow rate }
\end{aligned}
$$

Several assumptions separate the above equation from the governing equation (11) shown bellow, which only assumes that gravity is the only body force acting on a fluid particle and that the heat transfer into or out of the fluid is negligible. This equation is derived from a control volume analysis for a Lagrangian fluid element. 


$$
\Psi=\iiint_{C V} \Phi d V=-\frac{t}{d t} \iiint_{C V}\left(g h+\frac{1}{2} z^{2}\right) p d V \quad-\oiint_{d V}\left(g h+\frac{1}{2} z^{2}\right) p z_{z} \cdot d \lambda+\iiint_{C V} P(d i v z) d V
$$

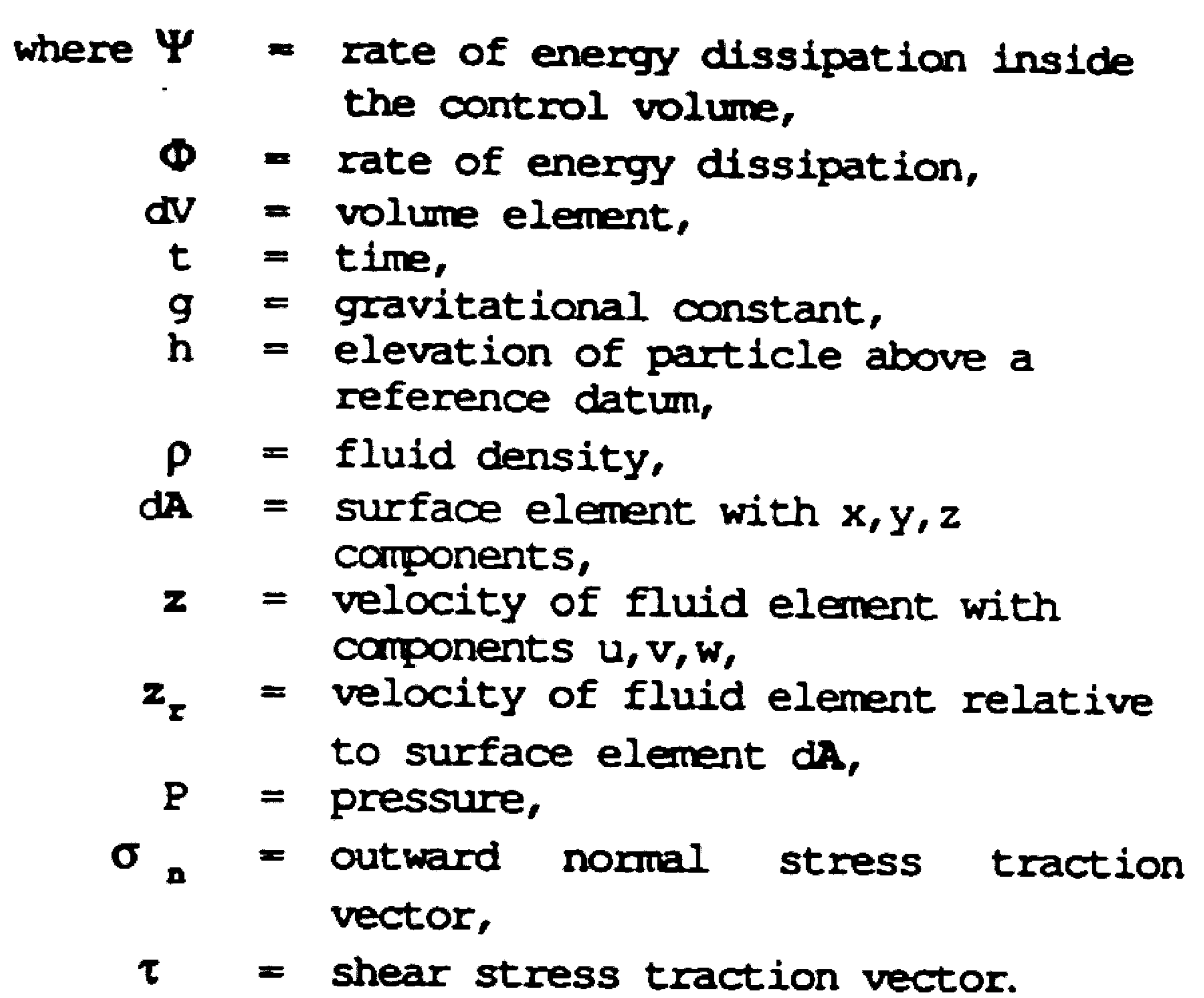

Equation (11) states that the rate of energy dissipation inside the control volume is equal to the decrease in internal energy in the control volume plus the decrease in rate of work of compression plus the rate of work done on the surface of the control volume. Two quite realistic assumptions allow the first and third term on the right to be cancelled: since conditions within the system are constant between cycles, the temporal variation term can be eliminated; and since the fluid is incompressible, div $z=0$, the third term vanishes. Equation (12) results.

$$
\Psi=-\oiint_{C V}\left(g h+\frac{1}{2} z^{2}\right) p z_{z} \cdot d A+\oiint_{C V}\left(\sigma_{n}+\tau\right) \cdot z d A
$$


The control volume is separated into two types os sections: walls and openings. The walls are assumed to be either rigid or perfectly elastic, such that no net work is done in either direction, from the wall to the fluid or from the fluid to the wall. The openings are the inlet and outlet of the new control volume; in such a way, equation (13) is generated.

$$
\Psi=-\iint_{I N / O U T}\left(g h+\frac{1}{2} z^{2}\right) p z_{z} \cdot d A \quad+\iint_{I N / O U T}(\sigma+\tau) \cdot z d A
$$

Assuming the fluid to be Newtonian (a fair assumption at the shear rates higher than $50 \mathrm{sec}^{\wedge}-1$ found in the ventricle and through the valves), the convective acceleration being negligible in comparison to the pressure term, and the density being constant, equation (14) is generated; which, when integrated with respect to time on both sides produces equation (15).

$$
\begin{aligned}
& \Psi=-\iint_{\text {IN/OUT }}\left((\rho g h+P)+\frac{1}{2} \rho z^{2}\right) z \cdot d A+\iint_{\text {IN/OUT }} \tau \cdot z d A . \\
& S=-\int_{0}^{I}\left(\iint_{I N / O U T}\left(P+\frac{1}{2} p z^{2}\right) z \cdot d A+\iint_{I N / O U T} \tau \cdot z d A\right) d t,
\end{aligned}
$$

where $\boldsymbol{S}=$ total energy dissipated per period $\mathrm{T}$

$\mathbf{P}=$ piezometric pressure $(\mathrm{P}+$ pgh) . 
The next three assumptions made are not considered accurate for the analysis of heart valves, but they are necessary because the measurements that would be required to account for the terms eliminated by these assumptions are not practical at this time. Equation (16) results from (15) by assuming that the flow is always normal to the inlet and outlet sections, thus eliminating the shear force term.

$$
S=\int_{0}^{T}\left(\iint_{I N}\left(P+\frac{1}{2} p z^{2}\right) z d A-\iint_{O U I}\left(P+\frac{1}{2} p z^{2}\right) z d A\right) d t
$$

It is next assumed that the piezometric pressure is constant throughout the cross section, giving rise to equation $(17)$

$$
S=\int_{0}^{T} \Delta P Q d t+\frac{1}{2} \rho \int_{0}^{T}\left(\iint_{I N} z^{3} d A-\iint_{\text {OUT }} z^{3} d A\right) d t
$$

Obviously not true, it is lastly assumed that there is no change in mean velocity profile between the inlet and outlet sections, allowing for the elimination of the last two terms on the right and the generation of the working equation (10).

$$
\varsigma=\int_{0}^{T} \Delta \mathbf{P} Q d t
$$


It is evident that the use of equation (10) as shown will not yield a parameter that accounts for the regurgitant loses of the valve. Hwang [35] proposed a phase-by-phase measurement of the power loss (equation (1) divided by the period) through the valve. As stated in his paper:

To utilize equation (1), we divide the
cardiac cycle into four phases and
categorize the power losses respectively
as: (1) the positive ejection phase power
loss (+EPL), (2) the negative ejection
phase loss (-EPL), (3) the regurgitation
phase power loss (RPL), and (4) the
leakage phase power loss (LPL).

As applied to the aortic valve, phase (1) is the power lost during the time when ventricular pressure is higher than aortic. During phase (2), the situation is reversed, aortic pressure is higher than ventricular, the fluid is moving along an adverse pressure gradient because of the inertia acquired during phase (1). Since the pressure drop for this phase is negative, Hwang assumes that this represents work being returned from the walls to the fluid and subtracts the power loss associated with this pressure 'gain' from that incurred during phase (1).

There is a distinction between phases (3) and (4), even 
though the flow through the valve is negative, retrograde, during both phases. The summation of the flows of phases (3) and (4) is usually referred to as regurgitant flow. Phase (3) sees some fluid pass through the valve, in the retrograde direction, during its closing; while, once fully closed, some fluid may still pass through the valve, but that is considered a leak, phase (4). Figure 54 shows a diagram of the four phases of the systolic period.

It is evident that multiplying the transaortic pressure drop during diastole (phases 3 and 4 ) by the retrograde flow cannot be equivalent to the power lost as a result of regurgitation. In addition to being physically incorrect, such procedure produces the incorrect conclusion that, if there is no valve at all, the pressure (across where the aortic valve would be during diastole, a period that should find this valve fully closed) drop will be 'zero' thus the power lost will also be 'zero' even though there will be no net flow, hence no net power delivered to the system.

It is also not correct to subtract the supposed energy gain of phase (2) from the losses of phase (1). For one thing, it is already assumed in the simplification of the working equation that the walls are either rigid or totally elastic. If the losses incurred during phase (1) are in the form of irrecoverable heat, viscous in origin, where does the power that is being returned to the flowstream during phase (2) come from? 
Hwang's phase-by-phase analysis of the losses through the valve by the method of Leefe is considered a major innovation that stands ready to be improved upon. The Energy Index method proposed here builds upon the labors of these two investigators while addressing the physical reality of the problem and producing a result that truly accounts for regurgitation $108 s e s$.

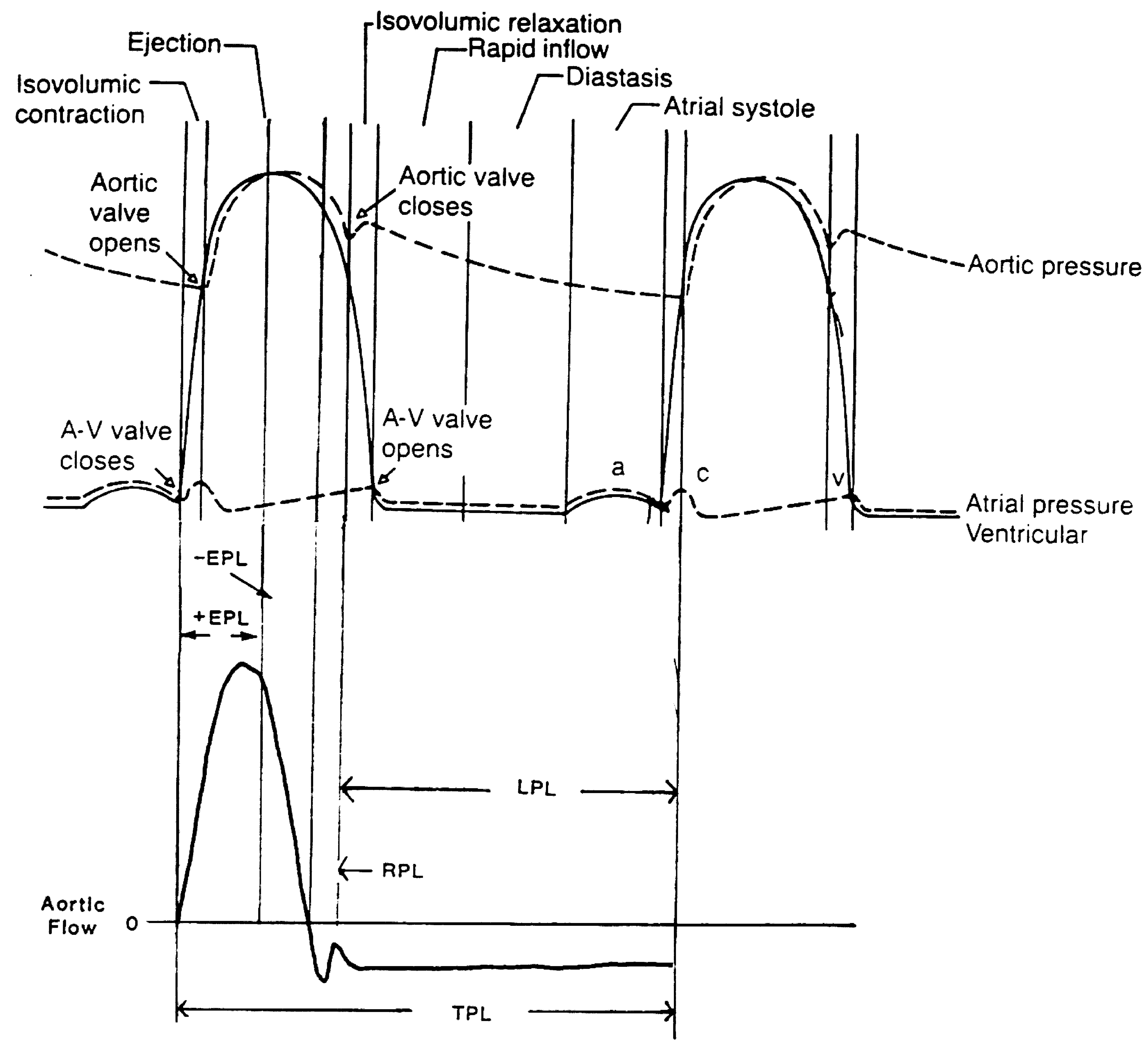

Figure 54: Schematic representation of the four phases of the cardiac stroke, after Hwang [35]. Figure includes pressure curves adapted from Guyton [27]• 
Because of the difficulties associated with the direct measurement of mitral flow rates, as explained in detail earlier, and since such measurement is essential for the energy index, the method was derived, and is described here, in terms of its application to the aortic valve. Later, the changes necessary to apply the method to the mitral valve will be described. Figure 55 depicts diagrammatically the model used to develop the energy index method.

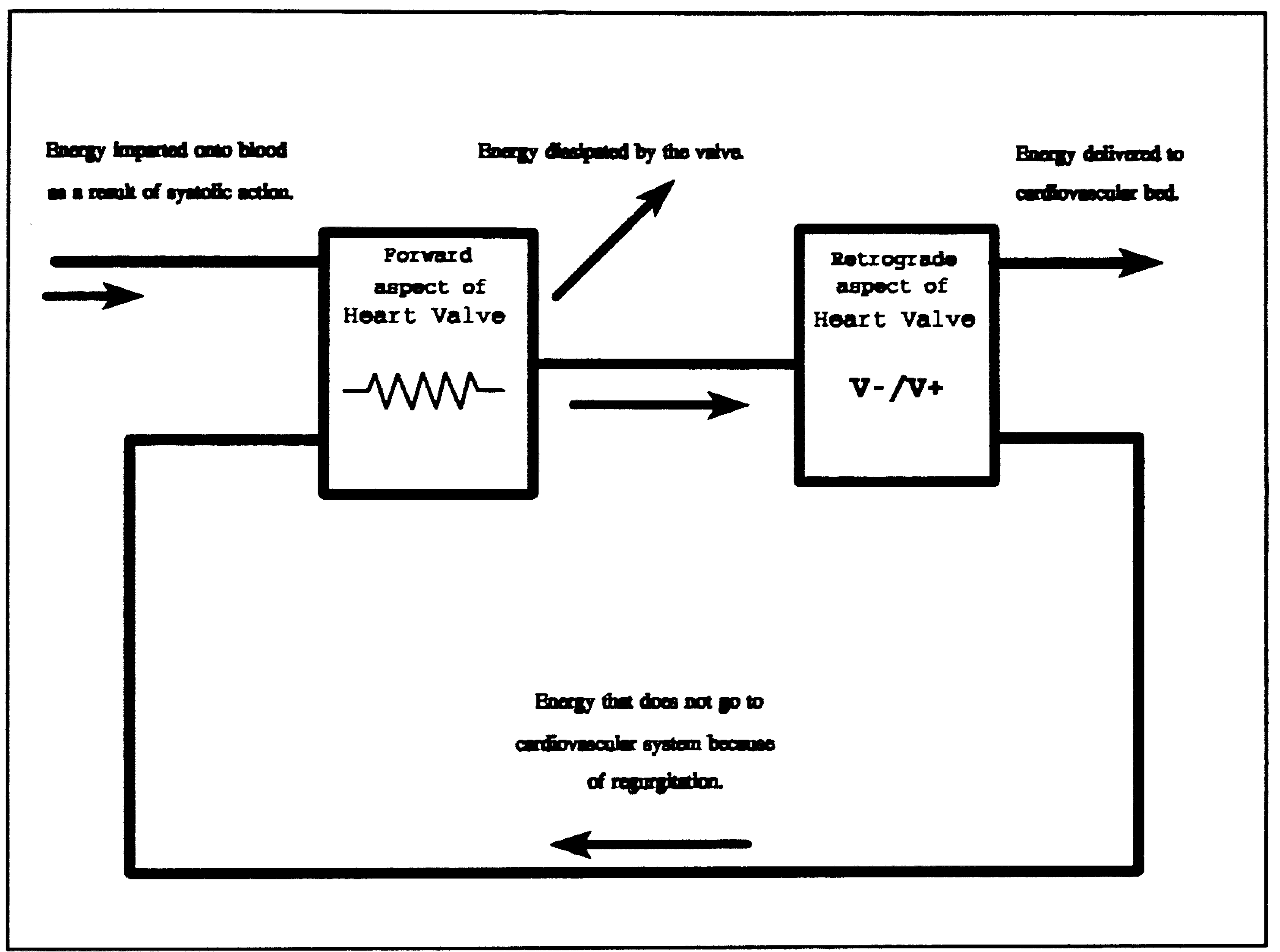

Figure 55: Schematic diagram used to derive the Energy Index method. 
Conceptually, this model is a simple case of conservation of energy. Its application to the aortic valve requires some investigation of the true meaning of the four phases of the systolic fraction described earlier. Energy is only dissipated in the valve when there is forward flow through it (during systole), this is the Ejection Phase Loss. Energy cannot be said to be dissipated in the valve during negative flow (during diastole). Instead, valvular regurgitation and leakage cause a reduction of the energy available for systemic irrigation; and, dissipation of additional energy when the regurgitated and leaked volumes pass through the valve again. The first energy mentioned is dissipated because the valve lacks a positive seal while closed, and because, unlike a normal natural valve, its closure requires some amount of negative flow. The second energy mentioned is dissipated in the valve as a result of the viscous and form losses during positive flow. The correct interpretation of the valvular phenomenon is crucial for the understanding of the energy index method, which is the ratio of the difference between the total energy generated and the energy dissipated as a result of forward and retrograde flow, to the total energy generated in one cardiac contraction.

$$
\text { Energy Index }=\frac{E(\text { generated })-(E(+f l o w)+E(- \text { flow }))}{E(\text { generated })}
$$

Further analysis is necessary to determine the meaning of 
each term of equation (18). The second term on the numerator is the energy dissipated during the first two phases of the cardiac cycle, described earlier. During the first phase, the energy dissipated by the valve is given by equation (1), which is the integral of the transvalvular pressure drop multiplied by the flow rate, during the period for which the ventricular pressure is larger than the aortic (the definition of the first phase). Both of these quantities are easily attainable in a laboratory by the methods described in previous chapters of this work.

If the same method, that is used for phase (1), of calculating energy dissipated by the valve was used for phase (2), the integral would have a negative value. This is one point at which the method presented by Hwang and Gross [35] is thought to not be correct: when this energy is subtracted from that dissipated during phase (1), two mistakes are being made: 1) the fact that energy is added to the flow at some station downstream of the valve, at any amount and from whatever origin, does not mean that the valve failed to dissipate energy during that phase. 2) the true energy dissipated during the second phase of the cycle is not accounted for. Even though the flow moves against an adverse pressure gradient, energy is still dissipated in the valve, hence it must be added to that energy that was dissipated during the first phase of the cardiac cycle. The measurement of the energy dissipated during the second phase would require that the 
pressure, at a location distal to where the valve is mounted, be measured with and without the valve's presence; evidently, if measured at the same temporal and spatial location within the cycle and the conduit respectively, the pressure has to be higher when the valve is not mounted than it would be for when the valve is mounted. This positive difference could, then be used with equation (10) to generate an energy lost during the second phase. Such procedure is impractical, however, specially where the natural valve and heart are concerned. An alternative solution that implicitly determines this energy is contrived in which the energy lost during the second phase of the cardiac cycle makes use of a cost function established during the first phase of the cycle. Since it is the case that flow through the valve alone causes energy to be dissipated, regardless of what the pressure gradient is during the flow, the energy dissipated by the valve during the first phase, divided by the effective volume of fluid that passed through as the energy was dissipated, establishes a cost function that can be used to determine how much energy is dissipated in the valve during the second phase of the cycle: the product of the cost function and the volume that passed through during that phase. Noting that the volume that passed through the valve is calculated from numerical integration of the measured flow rate curve, energy lost resulting from forward flow through the valve, $\mathrm{E}(+\mathrm{fl}$ low), is given by equation (19): 


$$
E(+f l o w)=E v 1+\frac{E v 1}{V 1} \times V 2
$$

where. Ev1 is given by equation (10)

$V 1$ is the volumed passed during the fust phase of cycle

$V 2$ is the volume passed during the second phase of the cycle

The use of such cost function presupposes that its value, the ratio between the amount of energy dissipated and the volume that caused such dissipation, remains constant throughout a range of volumes and/or energy amounts. While speculation on the subject is difficult at this point, it is noted that the range of volumes and amount of energy for which the cost function is meant to apply is very narrow, eliminating the possible detrimental effect of any nonlinearity of the ratio. The truth value of this hypothesis can be easily determined; since all of the experiments will be carried out with the same input flow wave forms and the same valve, a simple plot of the cost function as a function of either volume or energy delivered can be constructed and examined. Nonetheless, since each experiment has its own cost function, even if a non-linearity is found, causing differences in the cost functions of different experiments, the concept is still supposed valid.

The losses associated with phases (3) and (4) of the cardiac cycle are both of the same nature: they are incurred because of regurgitance, or negative flow through the valve. The segregation of regurgitant and leaking losses is only 
meaningful when carrying out an analysis of the valve's design, where the regurgitant volume is a function of the design and configuration of the valve, whereas the leakage is a function of the clearances and tolerances between the occluder(s) and housing. For the purpose of performance assessment, however, phases (3) and (4) can be lumped together as the negative flow phase.

As was the case with the ejection phase, the consequence of regurgitation must be understood before the energy dissipated during the third and fourth phases of the cardiac stroke can be quantified. Regurgitation causes a decrease in the amount of energy available for systemic irrigation; energy delivered (Ed), is decreased by an amount proportional to ratio of negative to positive volumes passed through the valve. The energy delivered to the system, Ed, is the integral of the flow rate multiplied by piezometric pressure, during the period for which flow is positive, measured at the aorta, just distal to the valve, but at a location that is not affected by local flow effects, according to the explanation offered in Chapter 2. The energy dissipated as a result of negative flow, $E(-f l o w)$, the third term on the numerator of equation (18), is:

$$
E(- \text { flow })=E d \times\left(\frac{-V}{+V}\right)
$$

where: $-V$ is the total retrograde volume

Ed is the energy delivered

$+V$ is the total forward volume 
The denominator, which is the same as the first term of the numerator, of equation (18) still needs to be defined in order for the Energy Index to be calculated. It is not possible to apply equation (10) to the ventricle because the flow there is not organized and confined to one stream, but the idea is to obtain the energy imparted on the flow as a result of systolic contraction, and unaffected by the presence and performance of the valve. Examining the systolic fraction of the cardiac stroke, it is noted that the second law of thermodynamics must apply; consequently, all of the energy generated must either be delivered to the system or be dissipated in the valve. Since the energy which is delivered to the system and that which is dissipated in the valve during the positive ejection phase have been described, thus being known, the energy generated is determined by the summation of those two amounts. Needless to say, this quantity is unaffected by the possibility of regurgitation because they happen at different phases of the cycle. Energy is only generated during the ejection phase, systole, and for that phase conservation of energy must apply. Eg is, then

$$
E_{g}=E v+E d
$$

where: Eg is the total energy generated by systolic contraction. (21)

$E v$ is the energy dissipated by the valve.

Ed is the energy delivered.

With the numerator and denominator of the equation for 
the energy index defined, it is noted that multiplication of the latter by 100 will yield a comfortable measure of the overall valvular effectiveness. Substitution of equations XYZ in to equation (9), and the necessary algegraic simplifications will produce a formal enunciation of the energy index:

$$
E I=\frac{E d \times\left(1-\left(\frac{V-}{V_{+}}\right)\right)}{E d+E v}
$$

Should the relative merits of a valve's stenoticity and regurgitance be desired, for the purposes of the analysis of its design, they can be calculated with the methods described here. Specifically, the stenoticity index would be the ratio of the difference, between the energy generated and the energy dissipated by the valve as a result of its stenoticity, to the total energy generated. The regurgitance index would use the same equation, but with the energy dissipated as a result of negative flow used in place of that dissipated as a result of positive flow, in the equation above.

$$
\begin{gathered}
\text { Stenoticity Index }=\frac{E(\text { generated })-E(+f l o w)}{E(\text { generated })} \\
\text { Regurgitance Index }=\frac{E(\text { generated })-E(-f l o w)}{E(\text { generated })}
\end{gathered}
$$


Needless to say, these indexes would only be useful for the engineering analysis of the valve's design, for they evidentiate the area that demands the greatest improvement. Were such indexes to be provided to the user of the device, the method would lose its simplicity merit.

It is noted that, as presented, the energy index method accounts for all facets that affect the performance of heart valves, while still producing a simple number to represent that effectiveness. The experiments described in the following section are designed to demonstrate the use and quality of the energy index method. As these experiments are carried out, it is expected that, provided the method is good, at a given heart rate (a perfect criterium for the clinician and the patient alike) the energy index of the valve decreases as regurgitance or stenoticity increases. Thus, prosthetic valves that are inherently more regurgitant or stenotic would, having a lower energy index, become readily exposed by a single number. 
Exporimontal Protocol

In order to test the effectiveness of the energy index method and to demonstrate its use, a set of thirty nine experiments were carried out with the same cardiovascular duplicator used for the previous set of experiments with the mitral valve and its Cd. Since the full potential of the energy index method requires knowledge of the regurgitant valvular flow, which can only be obtained with the electromagnetic flow meter, and since, at this time, the use of the meter available has not been possible for the determination of mitral flows - for reasons explained before the experiments were carried out on a $25 \mathrm{~mm}$ Tilting Disc valve mounted in the aortic position of the CVD. A $29 \mathrm{~mm}$ tilting disc valve was used in mitral position for all experiments. In addition to the difficulties associated with the precise determination of the positive and negative Mitral flow waves, the basic methods of Leefe and Gentle [34] and Hwang and Gross [35] were derived for application to the aortic valve. The energy index method introduced here, being an extension of the toil of these investigators, naturally begins with an investigation of the aortic valve. The appropriate modifications to the method, if it is to be used with the mitral valve, will be described later, nonetheless.

Every aspect related to the experiments, such as the instrumentation calibration, verification of results and 
equipment function, that was described earlier is still valid for these experiments. The only difference in the CVD for use with the experiments carried out to determine the energy index of the aortic valve under examination (only the performance of the $25 \mathrm{~mm}$ tilting disc aortic valve was studied), is that the flow probe was installed just downstream of the aortic valve, in order to capture the transvalvular positive and negative flow. The presence of the flow meter demands that salt be added to the fluid, as described earlier, but, because the amount needed is so small ( 0.98 by volume) the changes in viscosity and density of the fluid are negligible.

As the mitral valves used for the experiments with the orifice area method were made increasingly stenotic, the single aortic valve used here was given two degrees of regurgitance, such that, with the normal condition also used, a total of three sets of thirteen experiments were carried out. Regurgitance of the valve was increased by the preclusion of its full closure caused by a wire looped around the valvular housing. Although no specific degree of regurgitance was simulated, roughly 10 and $15 \%$, for the mild and severe cases of regurgitance respectively, were thought to be adequate. The normal regurgitant volume associated with the Tilting Disc valve is 3 to $5 \%$ of forward volume, increasing with decreased cardiac rate. Figure 56 shows the artifact used to induce regurgitation.

The quantities of interest for the energy index of the 
aortic valve are the transaortic pressure difference and the precise configuration of the flow wave form through the valve. With these pressures and flow curves the four phases of the cardiac cycle become evident, and equations $10,18,19,20$, 21,22 and 23 are readily applied.

The data from each of the thirty nine experiments was ensemble-averaged in the same way as described for the experiments with the mitral valve, for, nominally, twenty strokes of data were acquired. A spreadsheet (Quattro) was used to obtain all of the parameters of relevance, the results were exported to an Apple computer for effective plotting.

\section{Tilting Disc $25 \mathrm{~mm}$ \\ (Medtronic Hall)}

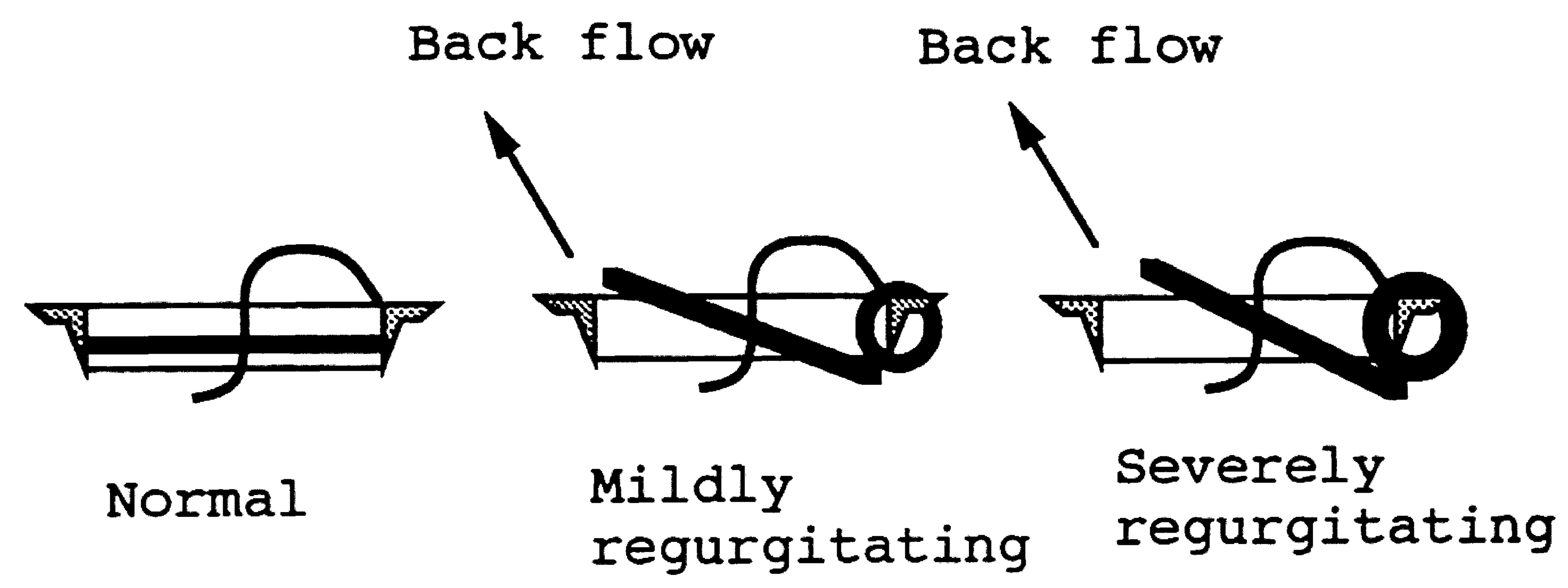

Metal rings used to induce abnormal regugitation

Figure 56: Diagrammatic representation of method used to induce regurgitation. 
The objective of the energy index method, as an assessment tool of valvular function, is to produce a single number that indicates the overall merit of the device as a valve. (noting that the ideal valve is that which demands no energy from the flowstream while open and closes with no regurgitated or leaked volumes)

An ideal performance index is that which can be described as a constant for a given valve, operating under any condition, unlike the effective orifice area method which requires a coefficient of discharge which may vary with valvular flow rate and transvalvular pressure drop. Having a single number to describe the performance of a valve is useful when comparing many models and sizes of prosthetic devices.

The determination of the constancy of the energy index for a given valve would require a more sophisticated experimental protocol, in which the EI is studied as a function of one variable at a time. Since the purpose of this set of experiments was simply to demonstrate the use of the energy index method, a complete evaluation of the method was not carried out; consequently, the EI is only described as a function of the cardiac rate, which happens to be a variable of large clinical relevance.

When the energy index is plotted as a function of heart rate, as shown in figure 57, it is observed that, for a normal 
valve, the energy index remains constant, when plotted as a function of heart rate, until the latter reaches values higher than 100 BPM. When measured between 50 and 100 BPM, a twofold increase, the EI remains nearly constant. The viscous and regurgitant losses associated with higher operational frequencies, and higher cardiac outputs, force the overall efficiency of the valve to decrease after roughly 100 BPM. Although not nearly as constant, when plotted as a function of the heart rate, the energy index for the aortic valve used is observed to decrease significantly with increased induced regurgitance. At this point, the method has already been shown to be sensitive to valvular condition; but no dependency, or lack of it, on cardiac frequency can be established since the input flow curves used are of such a nature that mean flow rates also increase with increased frequency.

Each column of figure 57 was produced from the average of three values for the EI produced for each heart rate. These three values originate from the three different stroke volumes associated with each cardiac rate, which gives rise to the twelve input flow functions whose parameters are described in table (1) (page 40). It is noted that table (1) was obtained by taking each "normal" cardiac output volume associated with its heart rate and increasing and decreasing it by 258 . The intention behind that maneuver was precisely the determination of the sensitivity of the performance parameter, in this case the energy index, to variations in stroke volume at a given 
heart rate. Although it is accepted that there can be no single physiological relationship between heart rate and stroke volume, it is also accepted that, a given heart, operates under very narrow variation of the proportion among the different parameters involved in the determination of the cardiac output. The use of cardiac outputs which are higher and lower than the "normal", by 258 at a given heart rate, may not be realistic physiologically. This speculation is offered as an attempt to explain the fact that, at 50 BPM, the energy index of the severely regurgitating valve, operating at a nominal output of $3.11 \mathrm{Lit/Min}$ and shown in figure 58 , is so much lower than the same amount for a cardiac output of 4.15 Lit/Min. Since a lower cardiac output, at the same heart rate, entails lower velocities (a smaller stroke volume ejected in the same amount of time), and since the induction of severe regurgitance is expected to change the delicate ventricular vortex structure, which is so crucial for effective flow out of the ventricle, and further, since the operation of the valves is mostly of an inertial nature, then, under the conditions under consideration, the valve cannot be expected to operate effectively and a net negative flow is possible. A negative net flow will produce an energy index which is negative. However, this does not mean that the system is creating energy; rather, it means that the energy which is being delivered to the system is flowing in the wrong direction. 
This difficulty is observed with the operation of the severely regurgitant valve operating at 150 BPM. In this case the net flow through the aortic valve was negative, indicating that the CVD was pumping fluid in the retrograde direction. Although this situation seems somewhat inconceivable, it is attainable under the condition imposed on the flow by the input flow function PS13. It was already noted that, under PS13 operation, the mitral valve was not operating effectively. Since there is less resistance to flow, during the onset of systole when both valves are still open, towards the atrium than towards the aortic root, a net negative volume is generated. The same explanation is true of the other input functions as well; however, in the more normal instances, since the stroke volume is much larger and the frequency of contraction lower, the effect of this phenomenon is diluted. Figures 58 and 59 show the energy index plotted as a function of the input stroke volume, for each heart rate. Figure 60 shows the same information for the simulated post surgical case. Comparing figures 58 and 59 to figure 57 , it is observed that the EI is more sensitive to changes in cardiac rate than for changes in stroke volume, at all three conditions of regurgitance. This is because these experiments simulated only increased degrees of regurgitance, but no stenoticity; and the fraction of regurgitated volume has been shown, by figure 61 (a) to increase with decreased heart rate. Experiments with stenotic valves are expected to produce a 
larger variation of the EI with stroke volume, because, in that case, more viscous losses, rather than regurgitating, will be observed. This increased viscous $108 s$ would appear, mathematically, as a higher value for the energy dissipated by the valve, Ev, which, being on the denominator of the equation for the energy index (equation 22), would cause the latter to be decreased.

Figure $61(\mathrm{~b})$ shows the net cardiac output plotted as a function of the heart rate. The very strong linear correlation between the two is not surprising since the operational parameters of Table 1 were defined this way. This figure is included here to show the quality of the measurements, which is evidentiated by the fact that the measured values are similar to the input flow functions. Figure 62 is included here for the same reasons, namely to demonstrate the repeatability of the measurements. Three sets of twelve data points, representing the energy generated as a result of ventricular contraction (which is unaffected by valvular condition) are shown superimposed on one another. Finally, in figure 63, the ratio of the energy dissipated (as a result of positive and negative flow) to the energy generated is plotted as a function of the cardiac rate.

The energy index method has been shown to be sensitive to valvular regurgitance and stenoticity, thus having potential as a parameter for the assessment of its function. Also, since the EI is a dimensionless number, it is easy to work with. 


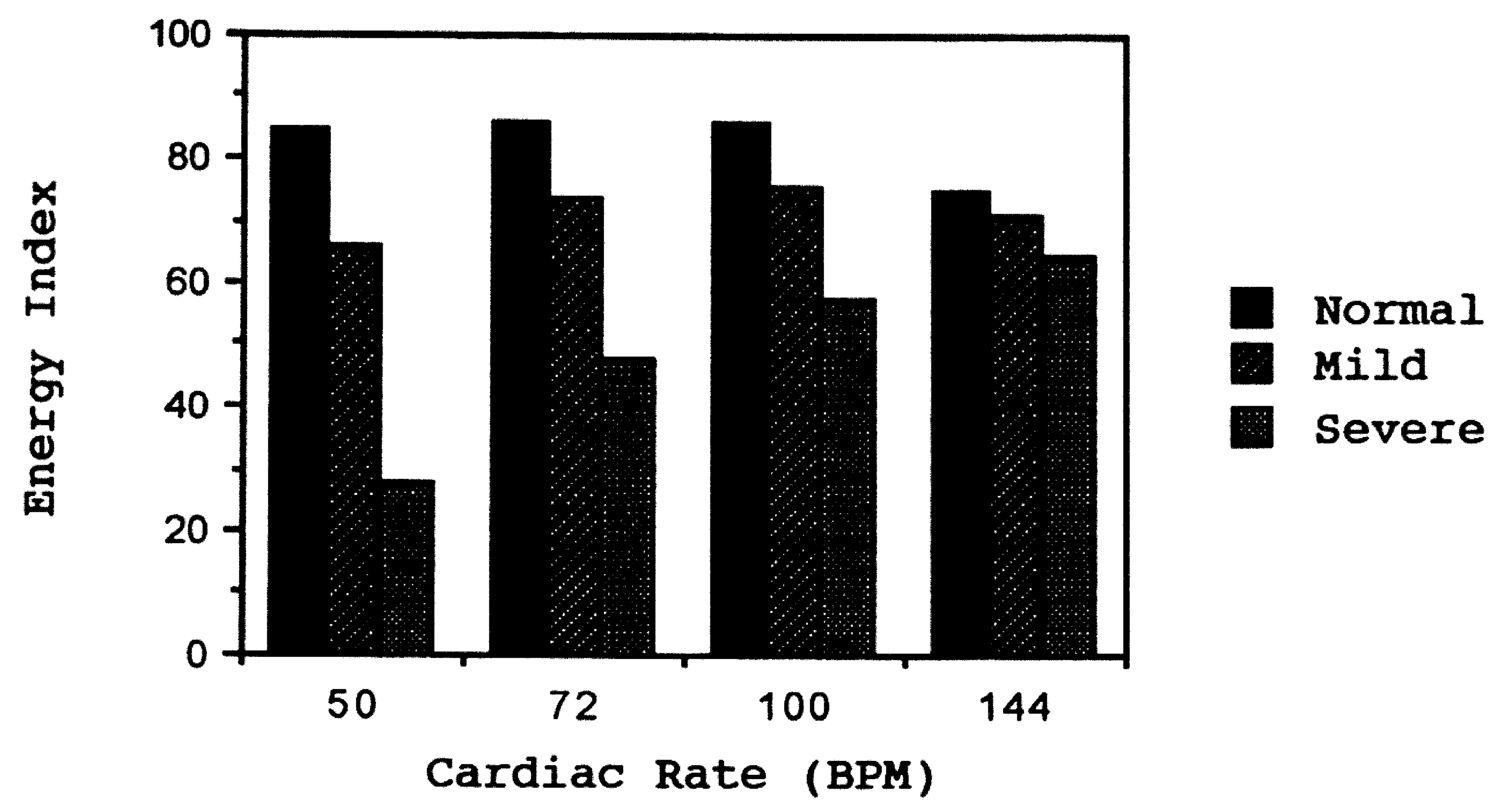

Figure 57: The energy index for valves operating under three degrees of regurgitance is plotted as a function of heart rate. 

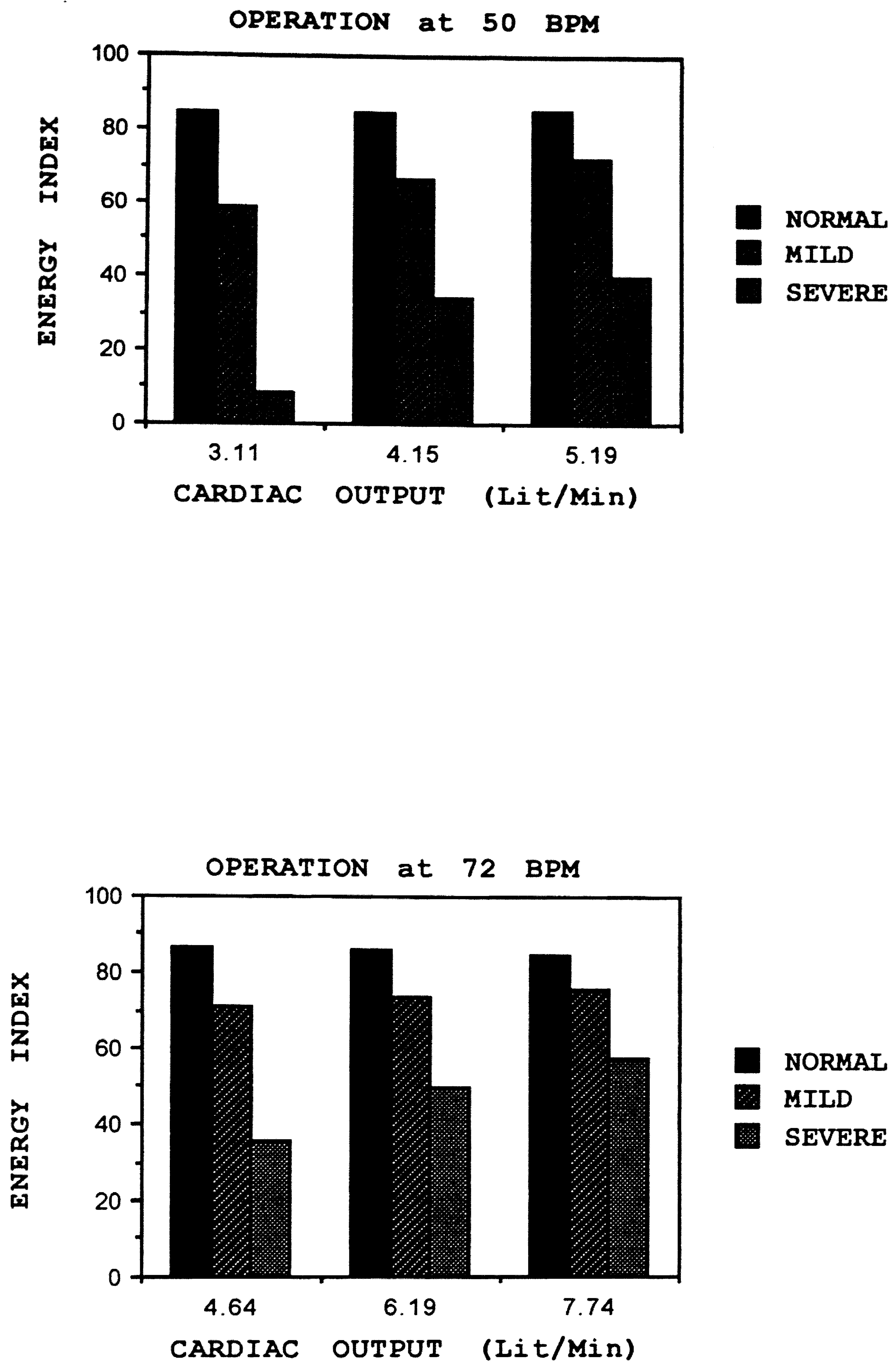

Figure 58: Energy index for heart rate of 50 and 72 BPM are plotted as a function of input stroke volume. 

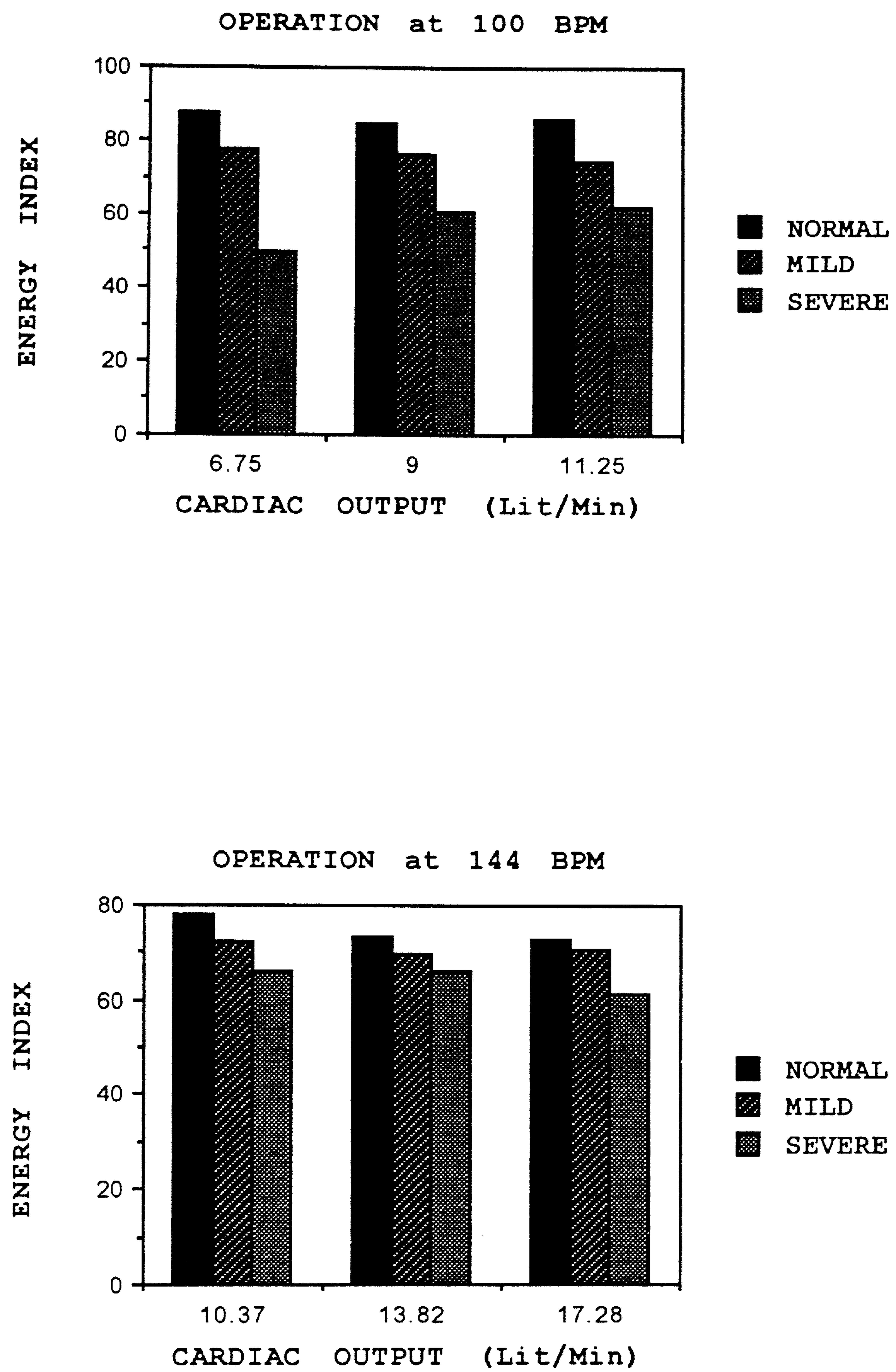

Figure 59: Energy index for heart rates of 100 and 144 BPM are plotted as a function of input stroke volume. 


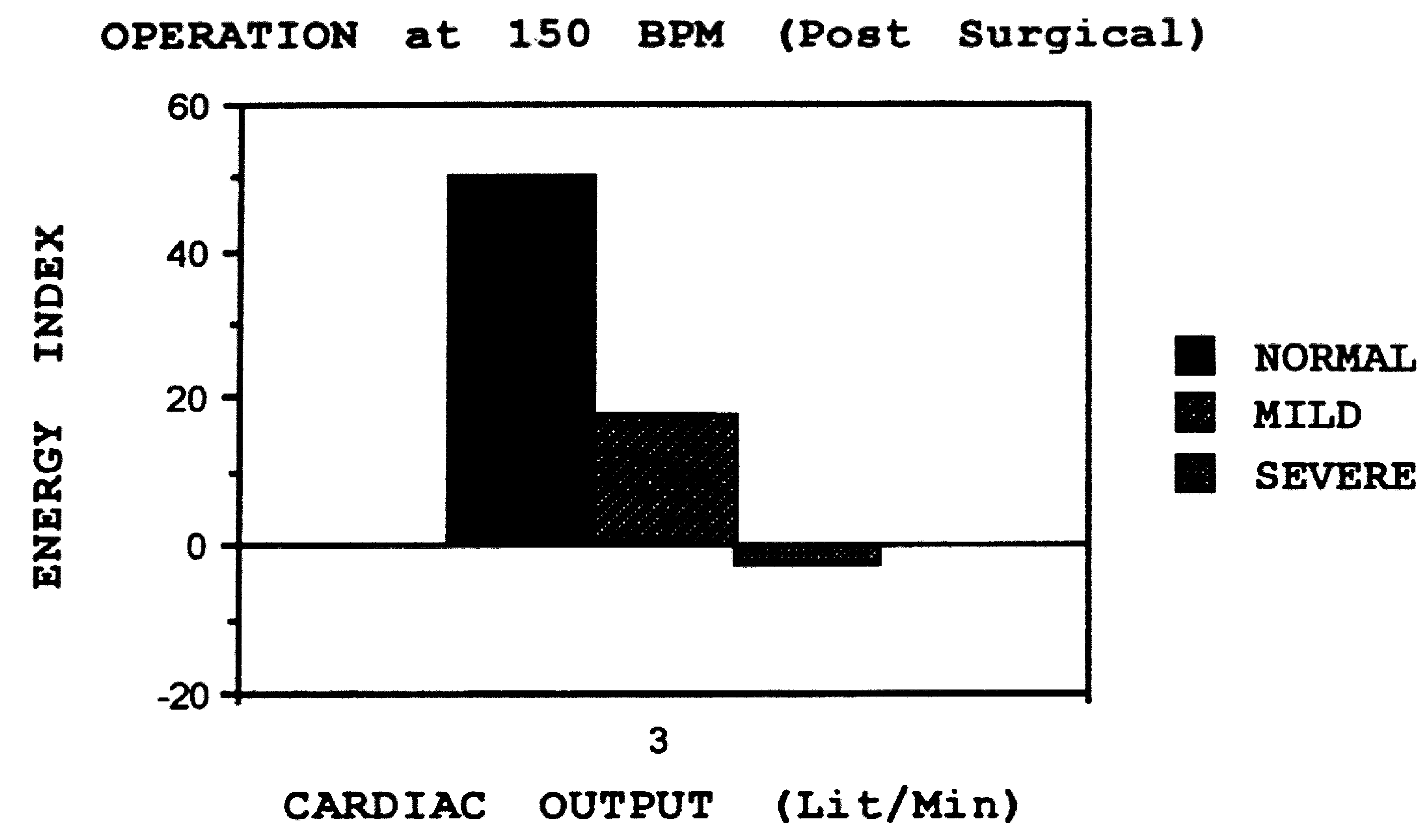

Figure 60: The energy index for the simulated post-surgical case is plotted for three valvular conditions of regurgitance. Nominal cardiac output is $3 \mathrm{Lit} / \mathrm{Min}$. Note that for severely regurgitant valve, net flow is in the reverse direction. 

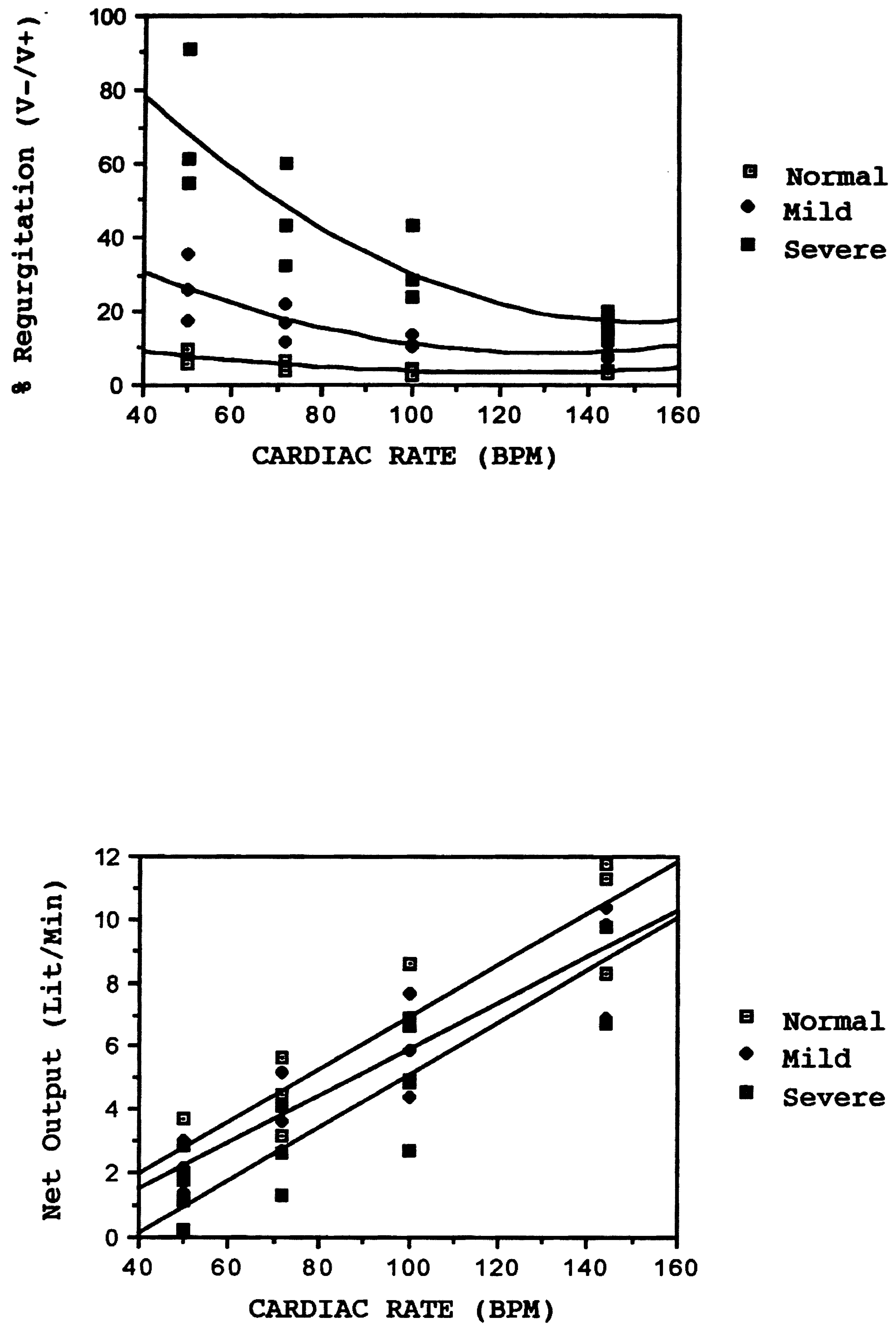

Figure 61: The fraction of the volume that is regurgitated is plotted against cardiac rate (top). Net cardiac output is plotted against the cardiac rate (bottom). 


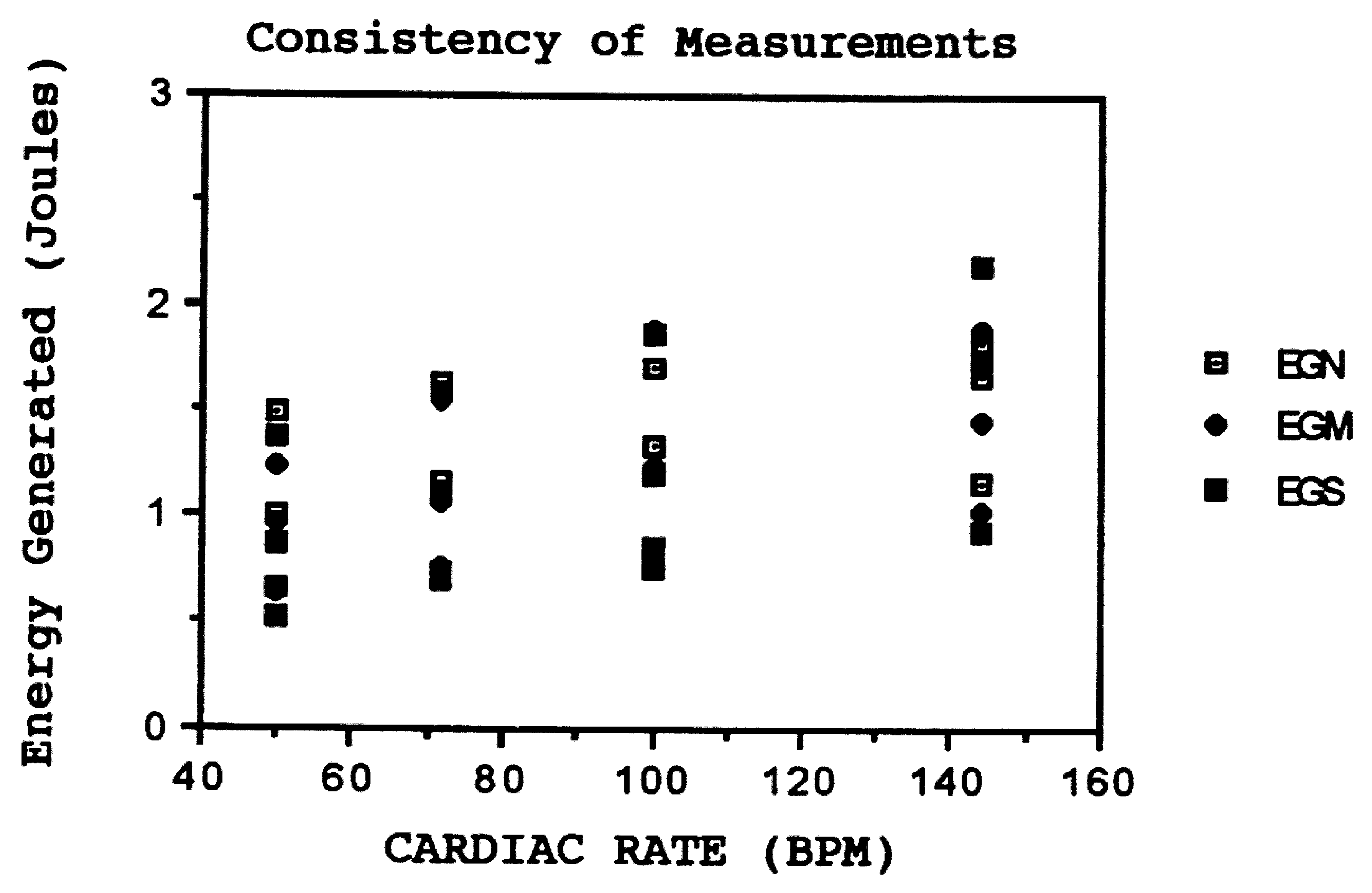

Figure 62: The consistency of the measurements is demonstrated by this plot. Unaffected by valvular condition, the energy generated is shown to nearly coincide for each of the three sets of measurements. 


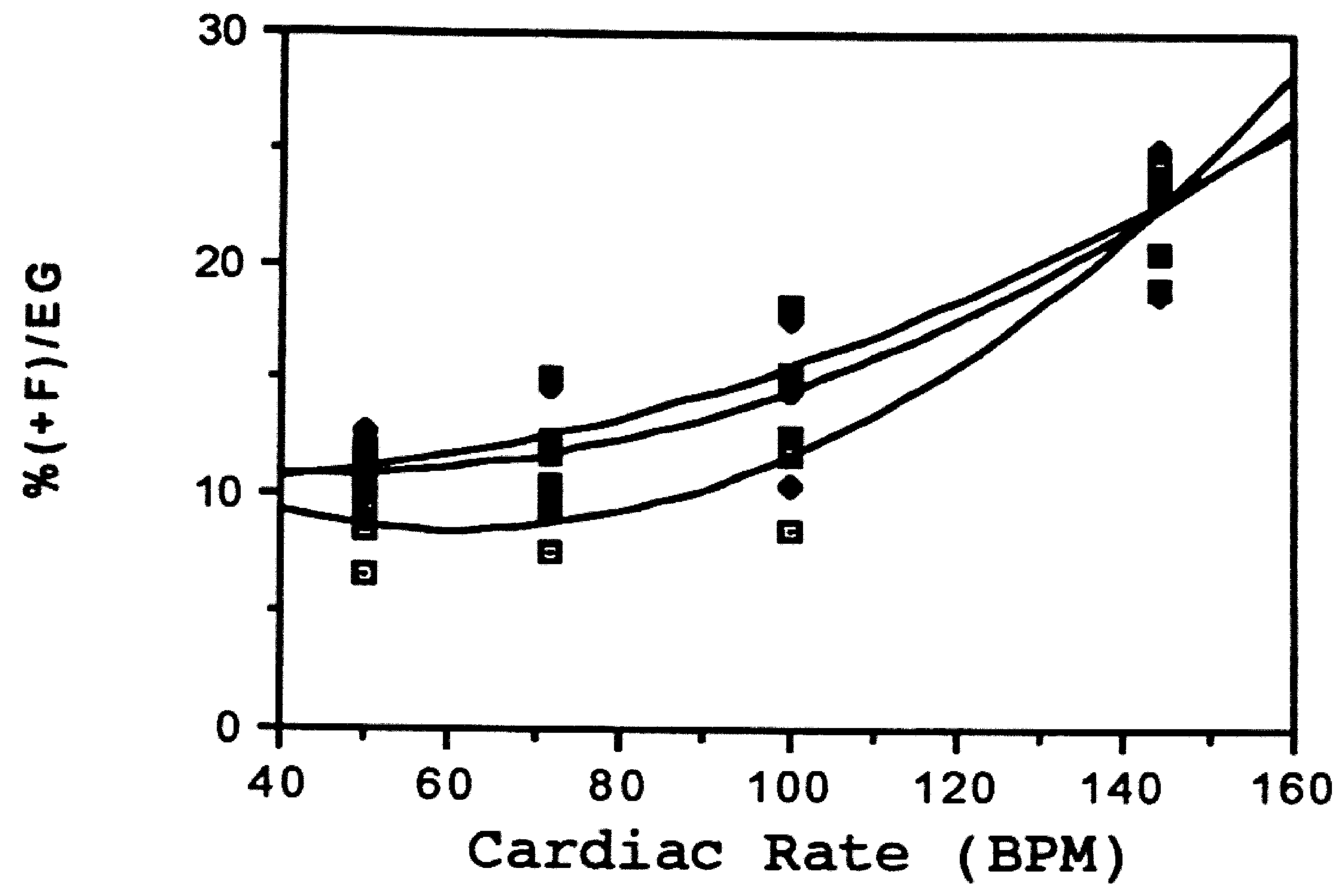

- Normal

- Mild

- Severe

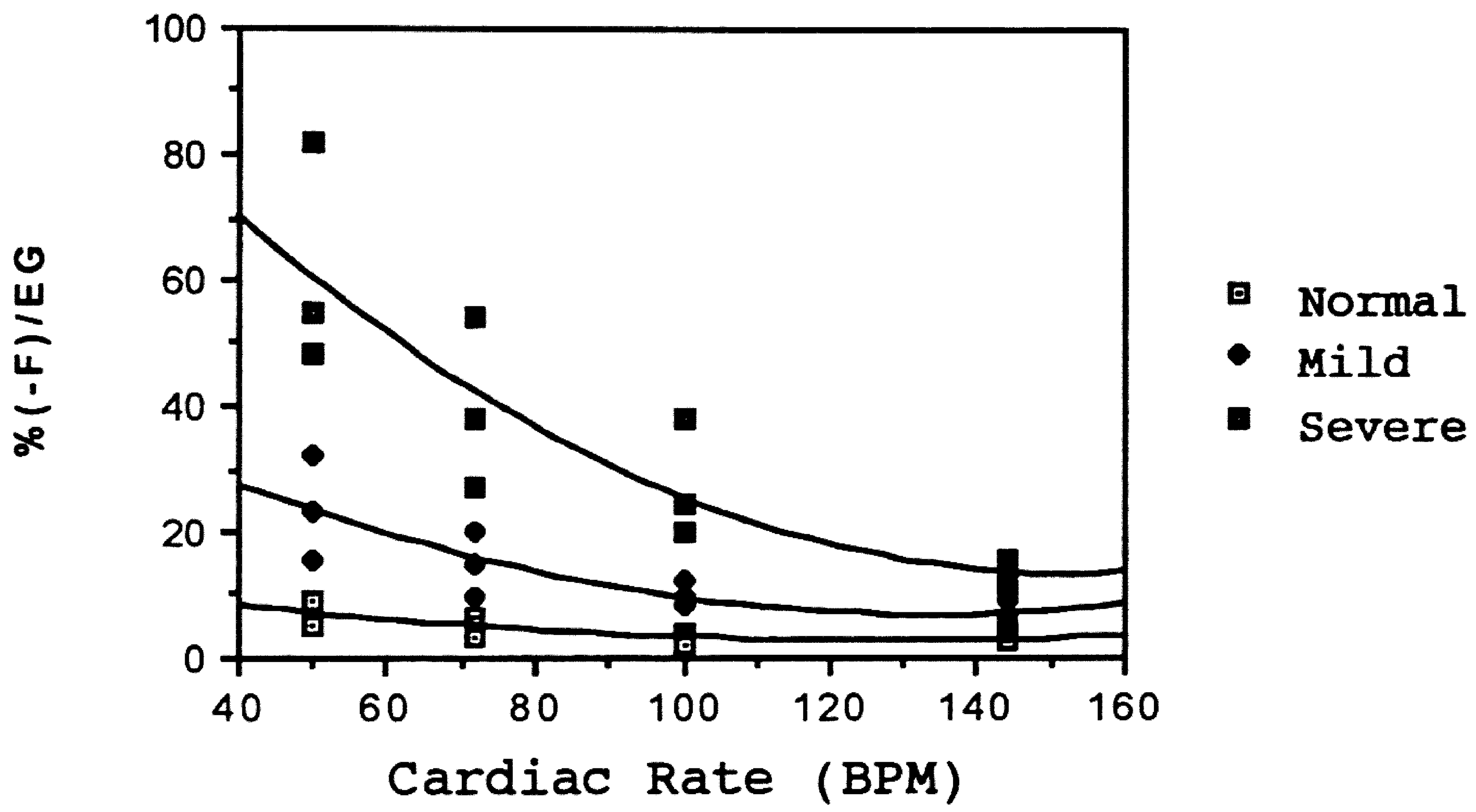

Figure 63: Ratio of energy dissipated as a result of forward flow (top) and negative flow (bottom) to the energy generated is plotted as a function of cardiac rate. 
The adequacy of orifice area methods of assessing heart valve function remains questionable primarily because regurgitant losses cannot be accounted for.

Although analysis reveals that the coefficient of discharge should be some linear function of the flow rate through the valve, no such correlation was found for the entire population of data points, for the population of each valve type, size or condition; even though pressure drops have been verified to increase with the second power of the Reynolds number.

In order to alleviate the difficulty associated with the determination of the $\mathrm{Cd}$, it is suggested that a simple correction factor $C f$ be used instead. This factor should be corrected for transvalvular flow rate and pressure drop, producing a surface instead of a line for its description.

The energy index method shows the potential to accurately predict valvular function, since it takes into account regurgitance and pressure drop losses. Furthermore, it may be stable over a wide range of conditions.

Future work that extends the envelope for which the energy index may be stable is suggested. 


\section{REEERENCES}

1) Gorlin, R. and Gorlin, S.G.: Hydraulic formula for calculation of the area of the stenotic Mitral valve, other cardiac valves, and central circulatory shunts, American Heart Journal. January 1951, Vol 41, No 1 .

2) She, L., Li, M., Wang, K., and Yu, Y.: Prosthetic heart valves: waveform comparisons and average value disposition for pulsatile flow in vitro. Journal of Biomedical Engineering. September 1990 , Vol 12 .

3) Nishimura, R., Housmans, P., Hatle, L., Tajik, J.: Assessment of diastolic function of the heart: background and current applications of doppler echocardiography. Mayo clinic proceedings No. 64: 71-81, 1989 .

$4)$ Chandran R.B.: Heart valve prosthesis in vitro flow dynamics. Encyclopedia of medical devices and instrumentation. New York: John Wiley and Sons, 1475-1483; 1986.

5) Pantalos G.M., Relf, W.J.: An improved mock circulation for total artificial heart instruction and performance analysis. Proceedings of the cardiovascular system dynamics society. VI th annual workshop. Philadelphia PA, 1984 • 68-71.

6.) Scotten, L. N., Walker, D. K., Brownlee, R. T.: Construction and evaluation of a hydro-mechanical simulation facility for the assessment of mitral valve prosthesis. Journal of Medical Engineering and technology. Vol. 3:11, 1979 .

7) Heimes, H.P. and Reul, H.: A new electronic device generating physiological flow in blood pumps and artificial hearts. 1973, page 98 of the Dig. of the 10th ICEMB, Dresden.

8) Reul, H., Minamitami, H. and Runge, J. 1975: A hydraulic analog

9) Heintze, Carl: The priceless pump. Thomas Nelson inc. New York, 1972

10) Myers, George and Parsonnet, Victor: Engineering in the Heart and Blood Vessels. Wiley-Interscience, New York, 1969.

11) Vassale, Mario, M.D.: Cardiac Physiology for the Clinician. Academic Press, New York, 1976.

12) Schoephoerster, Richard: Comparative Analysis of the Performance of Prosthetic Mitral Valves in a Model Left Ventricular Pulsatile System. A master's thesis by the author. University of Iowa. 1986. 
13) Schoephoerster, Richard: Flow dynamics and turbulence measurements past prosthetic mitral valves in a model left ventricle: experimental and numerical results. Doctorate thesis. University of Iowa, 1989.

14) Schoephoerster, Richard; Yearwood, Thomas; Chandran Krishnan: Prediction of Stenotic Valve Orifice Area: An in vitro study on a Bioprosthesis. Catheterization and Cardiovascular Diagnosis 18:36-47, 1989.

15) Schoephoerster, Richard and Chandran Rrishnan: Effect of Systolic Flow Rate on the Prediction of Effective Prosthetic Valve Orifice Area. Journal of Biomechanics, Vol 22. No 6/7, page 705-715, 1989 .

16) Aidley, David: The Physiology of Excitable cells. Cambridge University Press. New York, 1989.

17) Millar Instruments, Inc. P.O. Box 230227, Houston Texas, 77223-0227.

18) Swanson, W.M., Clark, R.E. (1977), Cardiovascular System Simulation Requirements. Journal of Bioengineering, 1 . Pp. 121-133.

19) Archer, S.G., Shmuel, E.,Sandor, A. (1978) Practical Aspects of Blood Flow Measurement. Statham Instruments, Inc. Oxnard, California. Page 8.

20) Weissler, A. M., Harris, W. S., Schoenfeld, C.D. (1968), Systolic Time Intervals in Heart failure in Man. Circulation, 37 , pp 419-519.

21) DuPlessis, L. A., Marchand, P. (1964), The Anatomy of the Mitral Valve and its Associated Structures, Thorax, 13, pp 221-227.

22) Minamitani, H., Reul, H., Runge, L. Tillmann, W. (1976), Effects of the Compliance of an Artificial Aortic Root on the Aortic Pressure Flow Patterns. Digest of the 11th I.C.M.B.E. Ottawa, Canada, pp 632-633.

23) Aaslid R., Levang O., Froysaker T., Skagseth E., Hall KV: In situ evaluation of the aortic pivoting disc valve prosthesis. Scandinavian J. Thorac Cardiovasc Surgery - 9:8184,1975 .

24) Gabbay S., McQueen DM, Yellin EL, Becker RM, Fratter RWM: In vitro hydrodynamic comparison of mitral valve prosthesis at high flow rates. J Thorac Cardiovasc Surg 76:771-786, 1978 . 
25) Black, M.M., Drury, P.J. and Tindale, W.B.: Twenty-five years of heart valve substitutes: a review. Journal of the Royal society of medicine, volume 76, August 1983. pp 667 .

26) Carolina Medical Electronics, Inc. King, North Carolina, U.S.A.

27) Guyton, A.C.: Textbook of Medical Physiology. 8th Edition. 1991. W.B. Saunders Company. Harcourt Brace Jovanovich, inc. The Curtis Center, Independence Square West, Philadelphia, PA, 19106.

28) Shepherd, J.T. and Vanhoutte, M.M.: The Human Cardiovascular System: Facts and Concepts. 1979. Raven Press, 1140 Avenue of the Americas, New York, New York, 10036.

29) Burch, G.E. and Giles, T.D.: Clinical evaluation of aortic and mitral valve. American Heart Journal, August 1976, Vol. 92, No.2, pp. 245-251.

30) Pacific Scientific, Motor and Control Division. 4301 Kishwaukee Street, P.O. Box 106, Rockford Illinois 61105-0106.

31) Omega Engineering, Inc. One Omega Drive, Box 4047. Stamford, Connecticut 06907-0047.

32) Westerhof, N., Gijs, E. and Sipkema, P.: An artificial arterial system for pumping hearts. Journal of Applied physiology. Volume 31, Number 5. November 1971.

33) Katz, A. M.: Physiology of the Heart. 1977. Raven Press, 1140 Avenue of the Americas, New York, New York, 10036.

$34)$ Leefe, S.E. and Gentle, C.R.: Theoretical Evaluation of Energy Loss Methods in the Analysis of Prosthetic Heart Valves. Journal of Biomechanical Engineering, 1987, Vol 9, April. pp 121-127.

35) Gross, J.M. and Hwang, N.H.C.: Energy Considerations of Prosthetic Heart Valves: An Initial Analysis. AIAA/ASME/SIAM/APS 1st National Fluid Dynamics Congress. July 25-28, 1988/Cincinnati, Ohio.

36) Cannon, S.R., Richards, K.L., and Crawford, M.: Hydraulic estimation of stenotic orifice area: a correction of the Gorlin formula. Circulation 71, No. 6, 1170-1178, 1985 .

37) Gorlin, R.: Calculations of cardiac valve stenosis: Restoring an old concept for advanced applications. Journal of the american college of cardiology. Volume 10, No. 4. October 1987: 920-2. 
38) Giersiepen, M., Rrause, U., Rnott, E., Reul, H. and Rau, G.: Velocity and shear stress distribution downstream of mechanical heart valves in pulsatile flow. The international Journal of Artificial Organs / vol. 12 / No. 4, 1989 / pp. 261-269.

39) Bruss, R.H., Reul, H, Gilse, J.V. and Knott, E: Pressure drop and velocity fileds at four mechanical heart valve prosthesis: Bjork-Shiley standard, Bjork-Shiley concaveconvex, Hall-Kaster and St. Jude Medical. 1983. European Society for Artificial Organs. Life Support Systems 1, 3-22.

40) Reul, H., Talukder, N. and Muller, E.W.: Eluid Mechanics of the Natural Mitral Valve. Journal of Biomechanics, Vol. 14, No. 5, pp. 361-372, 1981 .

41) Olin, C.: pulsatile flow studies of prosthetic aortic valves. Scandinavian Journal of Thoraxic cardiovascular surgery. 15: 1-12, 1971 .

42) Gilbert, D.B., Nolan, S.P., Stewart S., Fogarty, T., Harris, E.K.: An in vivo study of energy losses of ball and disc valves in the mitral position. Journal of applied physiology $28(3): 282-289,1970$. 